\title{
Investigation and Modelling of Rubber Stationary Friction on Rough Surfaces
}

\author{
Von der Fakultät für Maschinenbau \\ der Gottfried Wilhelm Leibniz Universität Hannover \\ zur Erlangung des akademischen Grades \\ Doktor-Ingenieur \\ genehmigte
}

Dissertation

von

Dipl.-Ing. André Le Gal

geb. am 09. Oktober 1978 in Ploemeur (F) 
Vorsitzender: $\quad$ Prof. Dr.-Ing. B.-A. Behrens

1. Referent: Prof. Dr.-Ing. G. Poll

2. Referent: Prof. Dr.-Ing. J. Wallaschek

3. Referent: Prof. Dr. R. H. Schuster

Tag der Promotion: 17.08.2007 


\section{Acknowledgment}

The following work has been carried out during my activity as research associate at the Deutsches Institut für Kautschuktechnologie (D.I.K.) between 2002 and 2006 in Hannover.

I would like to express my sincere gratitude to my thesis supervisor, Prof. Dr.-Ing. G. Poll, for his guidance throughout the course of my PhD.

I would like to thank Prof. Dr. R.H. Schuster for giving me the opportunity to carry out my $\mathrm{PhD}$ at the D.I.K. and being part of the commission as well as Prof. Dr.-Ing. B.-A. Behrens and Prof. Dr.-Ing. J. Wallaschek for their participation as commission members.

I warmly thank Dr. M. Klüppel for the fruitful collaboration during the last four years and his encouragements to accomplish this work. Also, I am grateful to Prof. Dr. G. Heinrich and Dr. T. Alshuth for introducing me into rubber technology and the fascinating field of elastomer physics.

I wish to express my sincere thank to my predecessors at the D.I.K. for their guidance during the firsts steps of this work: Dr. J. Meier, Dr. F. Abraham, A. Müller and Dr. M. Säwe. During this work, I have collaborated with many colleagues from the D.I.K. and the University of Hanover for whom I have great regards for their support at some stages and I wish to extend my thanks to all those who have helped, in particular within the Material Modelling and Concepts department.

A special thank is due to Dr. L. Guy from Rhodia for fruitful discussions on wet grip of tyres and rugby - as well as his decisive contribution regarding the two-scaling-regimes approach for the modelling of hysteresis friction.

The financial support of the Deutsche Kautschuk-Gesellschaft (DKG), Deutsche ForschungGesellschaft (DFG Forschergruppe: "Dynamische Kontaktprobleme mit Reibung bei Elastomeren“) is gratefully acknowledged.

My special gratitude is due to my family for their loving support, in particular my mother for her $50^{\text {th }}$ birthday this year.

I owe my loving thanks to my wife Eva Peregi for her constant encouragement and understanding during the accomplishment of this work. 


\section{Abstract}

This work deals with the investigation and modelling of rubber stationary sliding friction on rough surfaces. Through a novel physically motivated approach of dynamic contact problems, new insights in the understanding of rubber friction are achieved. This is of high interest for materials developers and road constructors regarding the prediction of wet grip performance of tyres on road tracks.

Improvements of contact mechanics are proposed within the frame of a generalized Greenwood-Williamson theory for rigid/soft frictional pairings. The self-affine character of rough surfaces leads to a multi-scale excitation of rubber during sliding process and the resulting hysteresis friction arises from material losses integrated over a range of frequencies. Beside a complete analytical formulation of contact parameters, the morphology of macrotexture is considered via the introduction of a second scaling range at large length scales, leading to a finer description of length scales that mostly contribute to hysteresis friction. On the other side, adhesion friction is related to the real area of contact and the interfacial shear strength which illustrates the kinetics of peeling effects distributed within the contact area at small length scales. This confirms well-known viscoelastic features exhibited by hysteresis and adhesion friction of elastomers on rough surfaces. The high frequency viscoelastic properties of filled elastomers are estimated by combining relaxation spectroscopy methods. As a result, a generalized master procedure is proposed for filled composites based on thermally activated processes of the bound rubber at the vicinity of filler particles above the glass transition temperature.

Friction investigations carried out under defined conditions show the relevance of hysteresis and adhesion concepts on rough surfaces. In particular, the use of a tenside as lubricant allows a quantitative measurement of both components. The model leads to satisfying correlations with friction results within the range of low sliding velocities with a significant improvement through the introduction of a second scaling range. In particular, the influence of polymer and filler type can be fairly well understood. Finally, the dynamic indentation behaviour of elastomers appears to be a promising route for further improvements in the modelling of rubber sliding friction.

Keywords: Rubber friction, self-affine surfaces, contact mechanics 


\section{Kurzfassung}

Im Rahmen dieser Arbeit werden die Reibeigenschaften von Elastomeren auf rauen Oberflächen auf Basis einer physikalisch motivierten Modellierung der Hysterese- und Adhäsionsanteile untersucht. Dabei wurde die Bedeutung der mikro- und makroskopischen Rauigkeiten der Reibflächen für den Reibkontakt und damit zusammenhängende Adhäsionsund Reibungsphänomene auf makroskopischer Längenskala aufgeklärt. Dies soll die Entwicklung neuer Materialien mit optimierten Reibeigenschaften unterstützen.

Experimentell wurden die Reibwerte bei unterschiedlichen Kontaktbedingungen im Bereich kleiner Geschwindigkeiten charakterisiert. Die Untersuchungen an den Modellsystemen haben gezeigt, dass die entwickelten Modelle zur Hysterese- und Adhäsionsreibung von Elastomeren auf rauen, selbst-affinen Oberflächen eine gute Beschreibung der experimentellen Reibdaten erlauben. Die Hysteresereibung spiegelt die Reibexperimente mit Seifenwasser als Lubrikant gut wider, da hier die Adhäsion durch einen tensid-stabilisierten Wasserfilm eliminiert wird. Die Trockenreibung lässt sich gut als Summe von Hysterese- und Adhäsionsreibung beschreiben, wobei der Adhäsionsanteil aus der wahren Kontaktfläche kombiniert mit der Grenzflächenspannung resultiert. Dadurch können typische Polymer- und Füllstoffeffekte auf die Reibeigenschaften vorhergesagt und physikalisch verstanden werden. Für weitere Entwicklungen des Modells stellt das dynamische Indentationsverhalten von Elastomeren einen interessanten Weg dar.

Stichworte: Gummireibung, selbst-affine Oberfläche, Kontaktmechanik 



\section{Table of content}

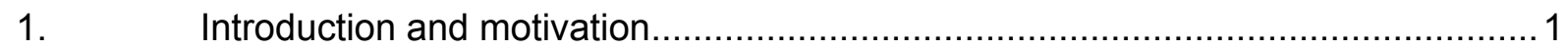

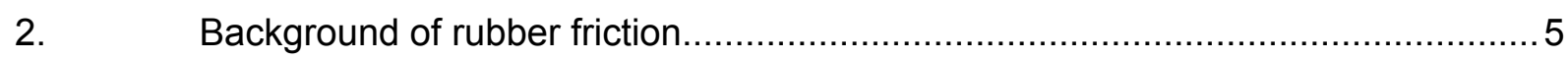

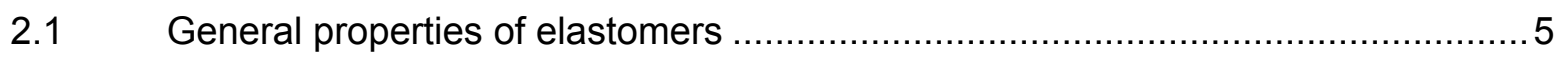

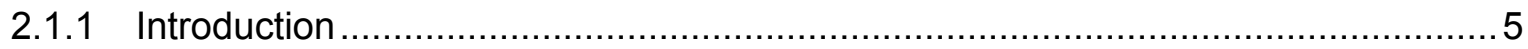

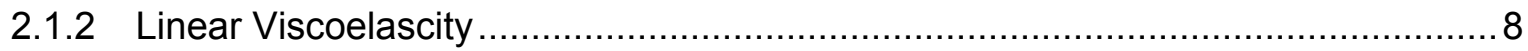

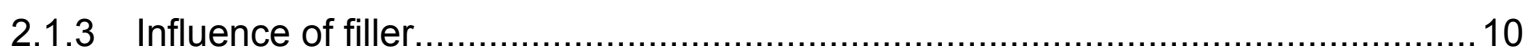

2.1.4 Time temperature superposition principle................................................ 11

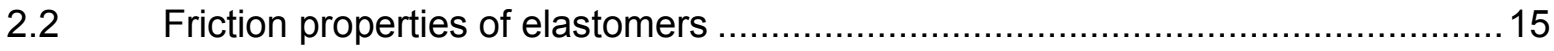

2.2.1 Physical mechanisms contributing to rubber friction....................................... 15

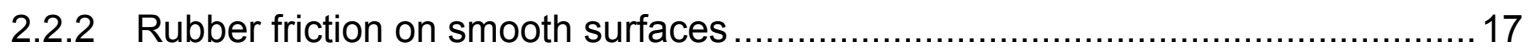

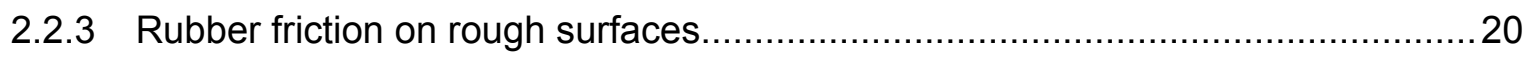

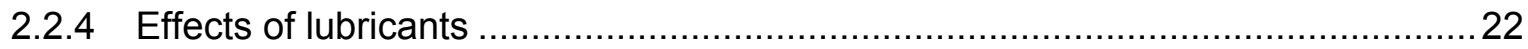

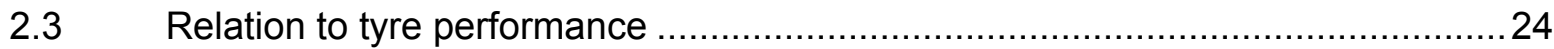

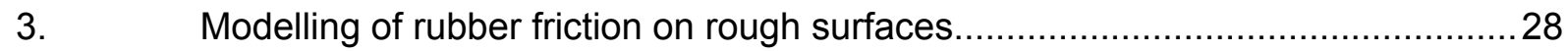

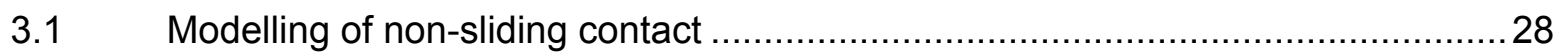

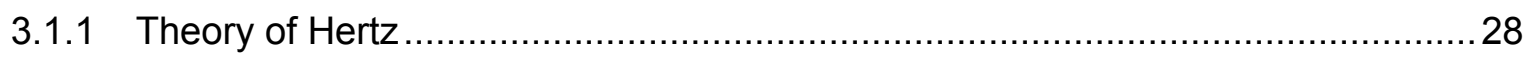

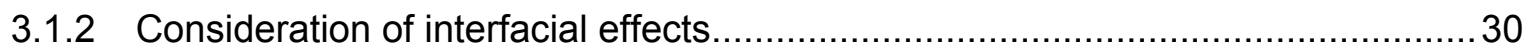

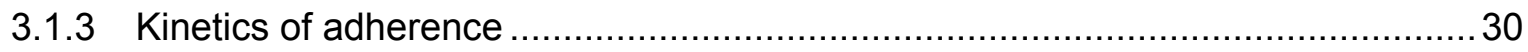

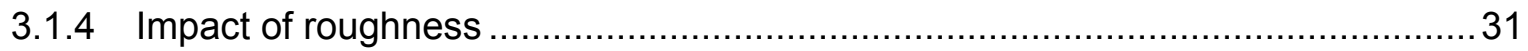

3.2 Contact mechanics on rough surfaces ....................................................... 31

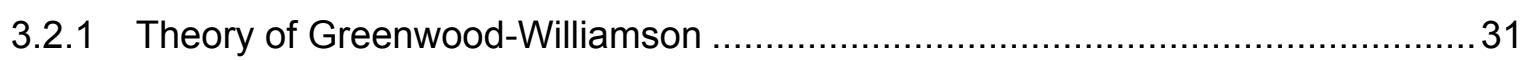

3.2.2 Extension of the Greenwood-Williamson formulation ........................................ 33

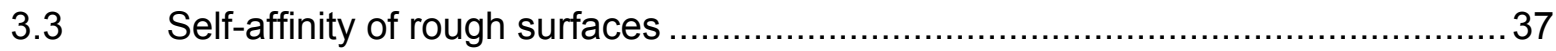

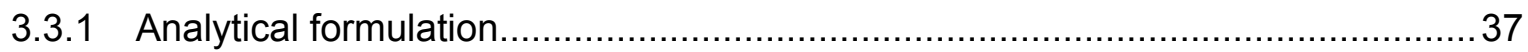

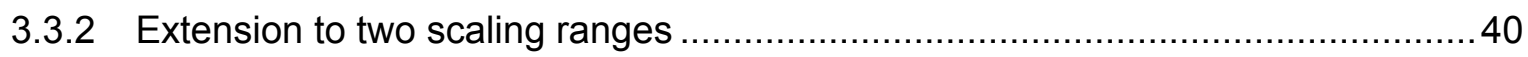

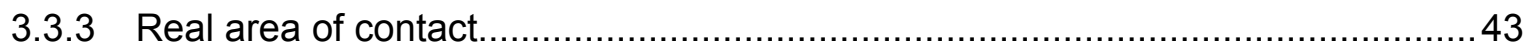

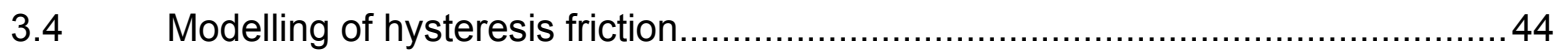

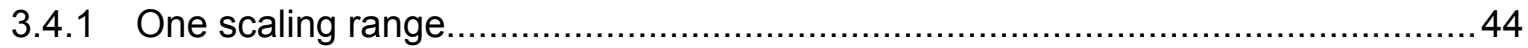

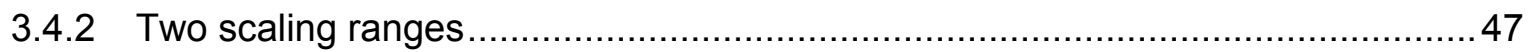

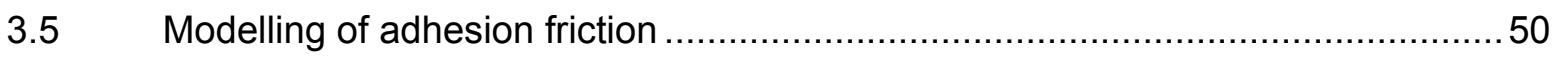




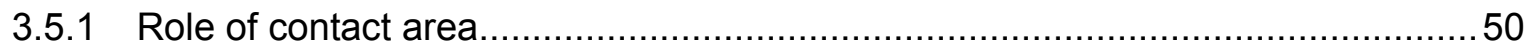

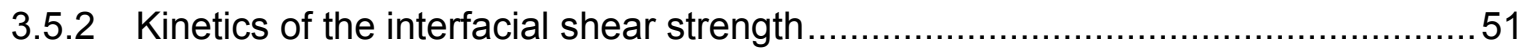

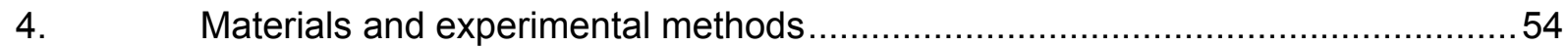

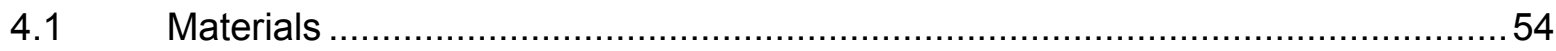

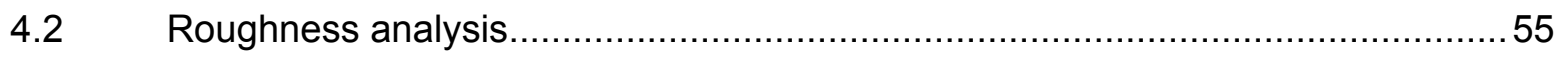

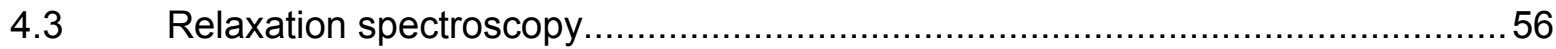

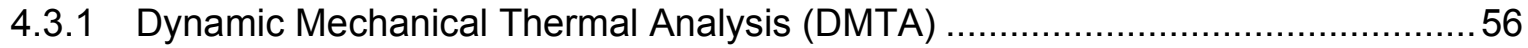

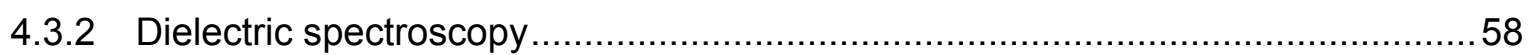

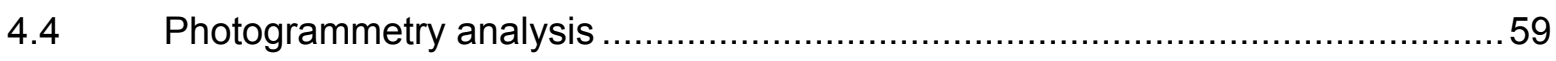

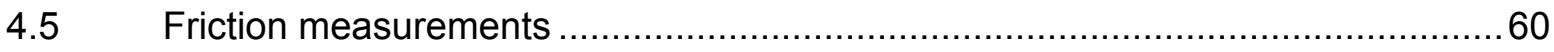

4.5.1 Modified Zwick universal testing equipment ..................................................60

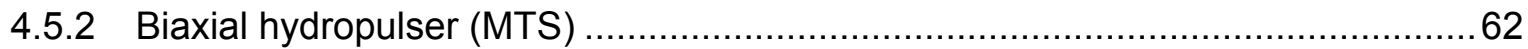

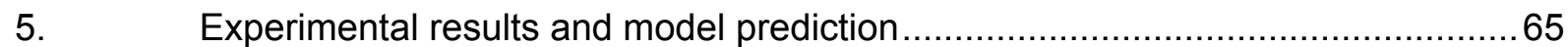

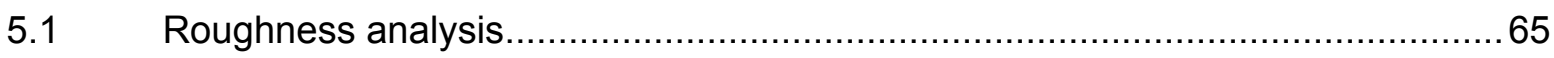

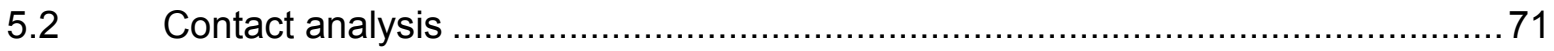

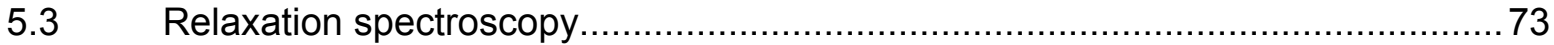

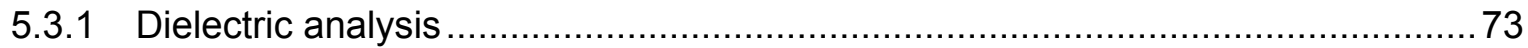

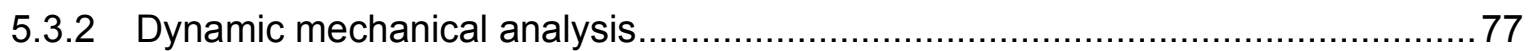

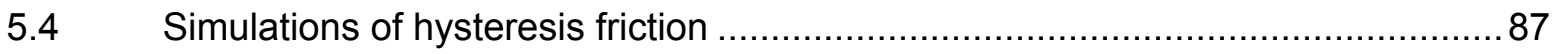

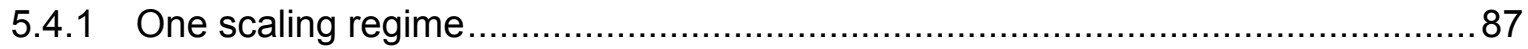

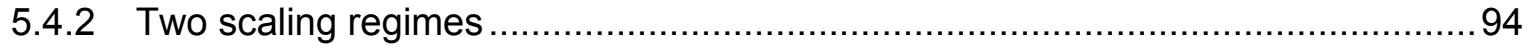

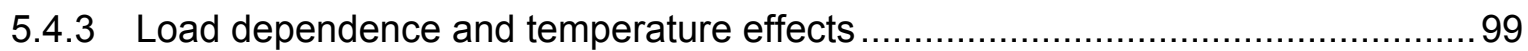

$5.5 \quad$ Correlations with friction measurements $-\mid$

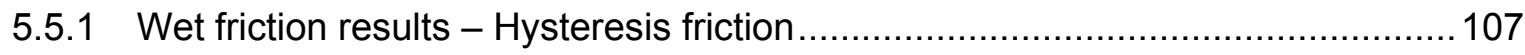

5.5.2 Difference dry / wet friction - Adhesion friction.............................................. 110

5.6 Correlations with friction measurements - II ............................................... 119

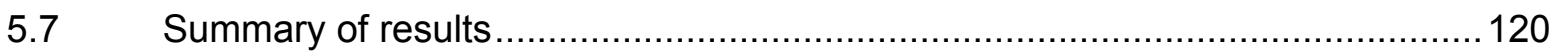

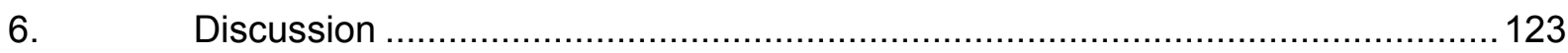

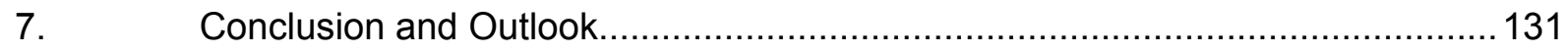

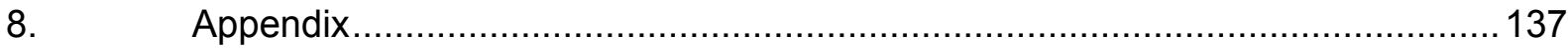

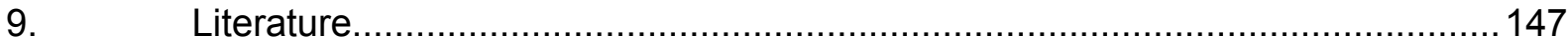




\section{List of symbols and abbreviations}
$A_{c} / A_{o}$
Ratio between real and nominal contact area
$\mathrm{C}_{\mathrm{z}}(\lambda)$
Height difference correlation function
$\mathrm{D}_{1}, \mathrm{D}_{2}$
Fractal dimension of macro- and microtexture
E'
Dynamic storage modulus (E' 3G')
E"
Dynamic loss modulus (E" 3G")
E-SBR
Poly(styrol-co-butadien), polymerised in emulsion
$\varepsilon$
Dynamic strain amplitude
$\varepsilon "$
Dielectric permittivity
$\mathrm{f}$
Frequency
$F_{0}, F_{1}, F_{3 / 2}$
Greenwood-Williamson functions
$\phi(z)$
$\mathrm{H}$
Height distribution of rough profile (index "s" for summit)
$\lambda$
Hurst exponent
Length scale
$\lambda_{\min }$
$\lambda_{2}$
$\mu$
Minimal length at which dynamic contact is realized
Boundary length scale between micro- and macrotexture
$\mu_{H}, \mu_{A}$
Friction coefficient
$\mathrm{S}$
Hysteresis and adhesion friction coefficient
Affine parameter of summit height distribution
S-SBR
Poly(styrol-co-butadien), polymerised in solution
$\mathrm{S}(\omega)$
Power spectrum density
$\tilde{\sigma}$
Standard deviation of height distribution (index "s" for summit)
$\sigma_{\circ}$
Load
$\mathrm{T}$
Temperature
$\mathrm{T}_{\mathrm{g}}$
Glass transition temperature
$\mathrm{t}=\mathrm{d} / \tilde{\sigma}$
Normalized distance rubber / mean value of rough profile
$\tan \delta$
Loss factor (E' / E')
$\tau_{\mathrm{s}}$
Interfacial shear strength
v
Sliding velocity
$\mathrm{v}_{\mathrm{c}}$
Critical velocity of adhesion friction
$\xi_{\perp}$
$\xi_{\|}$
Vertical cut-off length
Horizontal cut-off length 



\section{Introduction and motivation}

Friction is a fundamental physical phenomenon of high technological importance for a wide range of applications. Since friction arises from the relative motion of two bodies put in contact with one another, the effect is inherent to all mechanical systems involved in the transmission of forces or torques. Consequently, friction takes place in almost all components of power machines subjected to dynamic stresses, be it engine gears or during the contact wheel/rail or tyre/road. Thereby, the nature of frictional pairings is crucial for the description of dynamic contact problems.

Whereas the basic principle of friction was already used in the Antiquity, first concepts of friction are attributed to Leonardo da Vinci whose sketches have initiated the work of Amonton and the corresponding Laws of Friction. Later, exhaustive friction investigations carried out by Coulomb led to a mathematical formulation of the friction coefficient defined as the ratio between friction and normal force.

A wide variety of physical processes are associated with friction, for instance heat generation and abrasion. The transmission of accelerating or braking moments originates contact shear stresses with a subsequent increase of contact temperature that is directly related to the velocity gradient (slip), load and thermal conductivity of both materials, e.g. the ability to conduct heat away from the contact region. In addition, sufficiently high local contact stresses can lead to wear problems with a transport of material particles within contact region. Therefore, the investigation of mere friction problems requires the definition of confined experimental conditions which minimize the occurrence of the above mentioned unwished side effects.

\section{Rubber sliding friction on rough surfaces}

The particular case of rubber friction on rough surfaces displays a complex physical process but creates new challenges from the modelling point of view. This is due to the versatile thermo-mechanical behaviour of elastomers combined with the distribution of surface roughness over many length scales. As a result, the prediction of traction properties of tyres under wet conditions based on laboratory data stills remains an extremely difficult task. One 
reason is the insufficient analytical description of dynamic contact problems and the resulting friction phenomenon between elastomers and rough, rigid substrates.

Elastomers belong to the family of polymer materials, e.g. their microstructure basically relies on the entanglements of long macromolecular chains. They mainly differ from their glass transition temperature which is located below room temperature, indicating that elastomers exhibit a soft state at moderate temperatures under static conditions. One of the main advantages of rubber is that a wide range of target physical properties can be tailored by the addition of chemical components: a small amount of sulphur combined with high temperatures leads to the formation of a three-dimensional network with chemical bonds between polymer chains, e.g. significantly improves the elasticity. If fillers (carbon black, silica) are incorporated into the polymer matrix, dynamic mechanical and thermal properties are dramatically modified due to the occurrence of physical interactions associated with the filler network [6][7]. Hence the non-linear viscoelastic behaviour observed for filled elastomers under dynamic conditions.

When a rubber block slides on a rough substrate, the indentation process originating from surface asperities causes a periodical deformation of the elastomer related to internal losses. This energy dissipation mechanism induced during dynamic contact is denoted as hysteresis friction in the literature and found to be meaningful with increasing roughness amplitude. At the same time, the intimate contact down to small length scales suggests the occurrence of adhesive bonds. Thus, sliding friction can be seen as the successive formation and breakage of contact patches distributed over the nominal contact area which gives an additional contribution on the friction coefficient, namely adhesion friction. Since both components are associated with dynamic mechanical properties of elastomers, rubber friction on rough surfaces was found to exhibit typical viscoelastic features [13]. Consequently, rubber friction is expected to vary with sliding velocity, load, temperature, surface morphology and elastomer formulation. Moreover, the presence of lubricant at the interface has strong implications on the adhesion and prevents optimal contact at high sliding velocities through the occurrence of hydrodynamic effects.

Novel modelling of hysteresis and adhesion friction consider self-affine properties of surfaces, e.g. morphological invariance under anisotropic dilations, which means that surface roughness is considered over many length scales. The applicability of fractal concepts has been demonstrated for road surfaces leading to the establishment of empirical correlations between surface descriptors and traction properties of tyres during ABS-braking phases [63]. The consideration of self-affinity led to the recent development of hysteresis friction models 
in which energy losses generated by the roughness spectrum during dynamic contact are expressed as a friction integral over a range of excitating frequencies [59][60][61][48][49]. The evaluation of frequency interval is crucial for the simulated friction level and directly depends on the formulation of contact conditions.

A recent modelling of the contact between elastomers and rough surfaces has been presented by Heinrich and Klüppel [59][60][63]. Based on basic assumptions of Hertz and Greenwood-Williamson theories, rough surfaces are macroscopically approximated by spheres which are statistically distributed over a mean plane. The calculation of length scale dependent contact parameters like the real area of contact relies on scaling properties of rough surfaces down to the micrometer range. As a result, simulations of hysteresis friction could be achieved for filled elastomer on a silicium carbide surface and showed a qualitative good agreement with friction data obtained under lubricated conditions [71] (Figure 1.1).

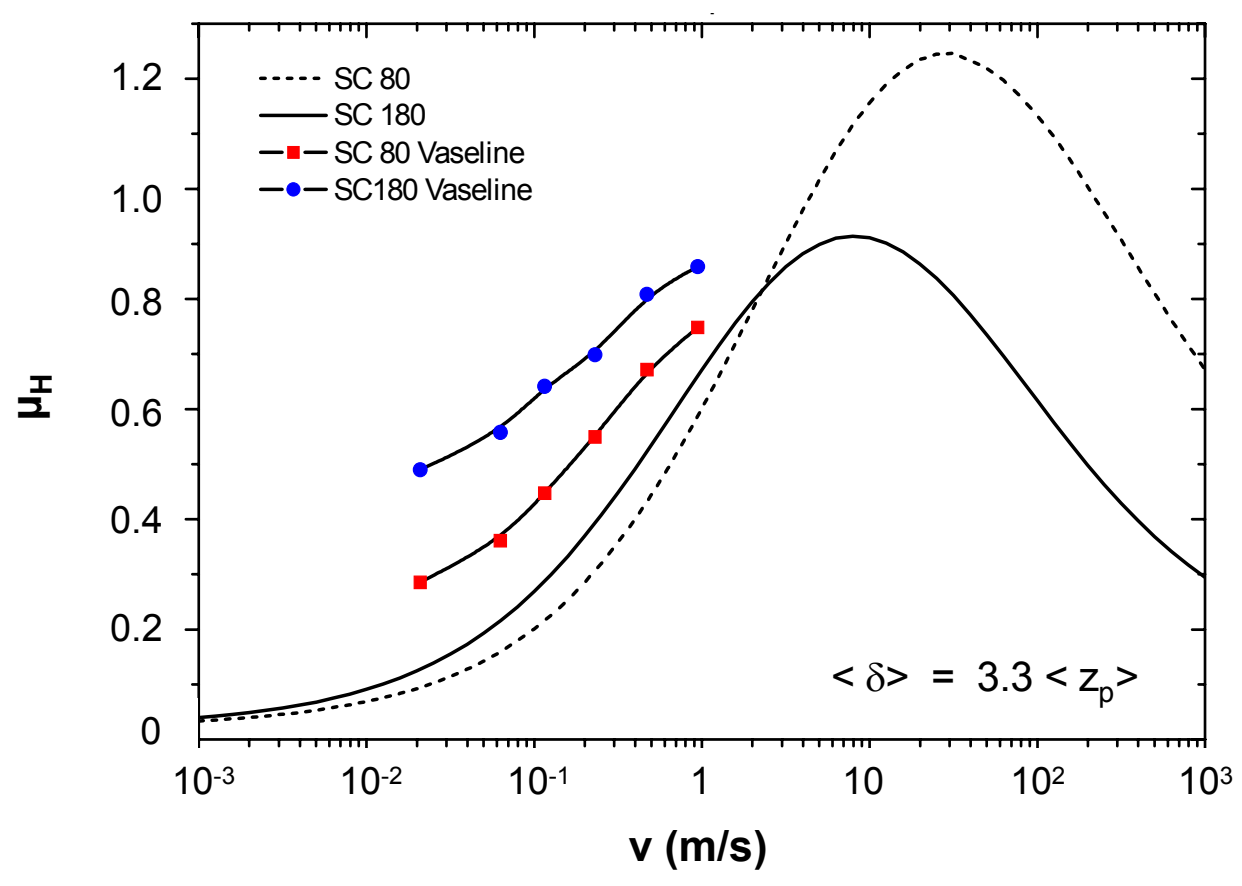

Figure 1.1: Friction results on lubricated silicon carbide surface for a filled S-SBR and associated simulations of hysteresis friction (from [71])

\section{Objectives and outline of the thesis}

The aim of this thesis is the modelling and analysis of microscopic influencing factors regarding stationary sliding friction and dynamic contact problems between elastomers and rough substrates. The description of sliding process on various length scales is of major 
concern for the comprehension of friction contact and therefore has to be clarified with respect to hysteresis and adhesion friction components. For this purpose, underlying concepts of contact mechanics and rubber friction are presented in Chapter 2. Beside basic considerations and an overview of existing approaches on rubber friction, the specific highfrequency behaviour of elastomers and its relationship to wet grip performance of tyres is pointed out.

Since a main focus of this work concerns the modelling of rubber friction, the entire Chapter 3 is dedicated to the presentation of a physically motivated theoretical approach for the description of hysteresis and adhesion friction during dynamic contact problems. This includes an extended formulation of contact mechanics determining the upper fraction of rough surfaces where contact actually occurs. This is of considerable importance for the calculation of contact parameters which are governed by the largest length scales of profile. Furthermore, the decomposition of roughness into two texture regimes leads to an extended formulation of the hysteresis friction with a quantitative estimation of both micro- and macrotexture during sliding process. In addition, adhesion friction is expressed as the real area of contact combined with a velocity dependent interfacial shear strength arising from the kinetics of peeling effects on small length scales.

Therefore, model elastomer formulations are prepared with a systematic variation of filler and polymer type (Chapter 4). They are based on different SBR grades mixed with modern filler systems like high dispersible silica incorporated with coupling agent. Next, experimental methods are presented in details regarding relaxation spectroscopy and friction measurements.

Chapter 5 presents a wide range of results, from the dielectric and dynamic mechanical analysis to friction data and the corresponding predictions of simulated friction. A novel master procedure is introduced for estimating the high-frequency behaviour of filled elastomers and further discussed on the basis of material microstructure. The impact of material and roughness parameters on simulated friction is exhaustively presented and furthermore compared to experimental investigations carried out under stationary contact conditions. The applicability of theoretical concepts is largely discussed in Chapter 6 and gives a deeper insight into length scale dependent physical mechanisms involved during rubber sliding friction on rough surfaces. 


\section{Background of rubber friction}

This chapter gives an overview on pioneering contributions that led to the establishment of a consistent physical description of rubber friction processes. First, the specific nature of elastomers is presented regarding dynamic mechanical properties and more generally the material behaviour within the high frequency region. The underlying concepts of linear viscoelasticity are reviewed in order to describe the temperature and frequency dependent relaxation behaviour of elastomers. This is followed by a detailed review of major scientific contributions dealing with rubber friction problems: the decomposition of the friction coefficient, the viscoelastic nature of rubber friction, as well as the impact of lubricant and roughness, are presented. Finally, the relevance of rubber friction properties is highlighted with respect to wet grip performance of tyres.

\subsection{General properties of elastomers}

In the last century, elastomers have been successfully used in a broad range of technological applications. Through the development of new generations of basis polymers and additives, the versatile behaviour of rubber could be extended in order to meet more demanding technological requirements. This section gives a deeper insight into the specific nature of elastomers with respect to relaxation properties.

\subsubsection{Introduction}

Elastomers are a specific type of polymer materials which differentiate through their ability to undergo large elastic deformations. Through polymerisation, long-chains flexible chainlike molecules can be assembled along a "backbone" constituted by covalent bonds around which short range motions are possible through thermal agitation [2][3]. Such molecules change their conformations continuously at normal temperatures and tend to adopt randomcoils rearrangement which corresponds to the most energetically favourable configurations (amorphous state). Under an external applied mechanical force, the amount of conformations is reduced and polymer chains are oriented in the direction of deformation. 
Each polymer chain is composed by a repetition of single units, the so-called monomers. The molecular structure of a monomer is of considerable importance as it determines the basic properties of rubber (processing, chemical stability, dynamic mechanical). Behind the Natural Rubber produced from the Hevea Brasiliensis and characterised by remarkable mechanical properties, general purpose synthetic elastomers have been discovered and then widely used for different applications within the last 100 years. They include styrene-butadiene rubber (SBR), butadiene rubber (BR) and synthetic polyisopren rubber (IR). These "diene" contain substantial chemical unsaturation in their backbones which make them susceptible to attack from oxygen and ozone. The molecular structure of a styrene-butadiene rubber is shown in Figure 2.1. SBR denotes a widely used copolymer of styrene and butadiene which is commercialized with different styrene grades. It can be synthesized via emulsion or solution polymerization, leading to a further differentiation between Emulsion SBR (E-SBR) and Solution SBR (S-SBR). The content of vinyl butadiene is directly related with the glass transition of the material.

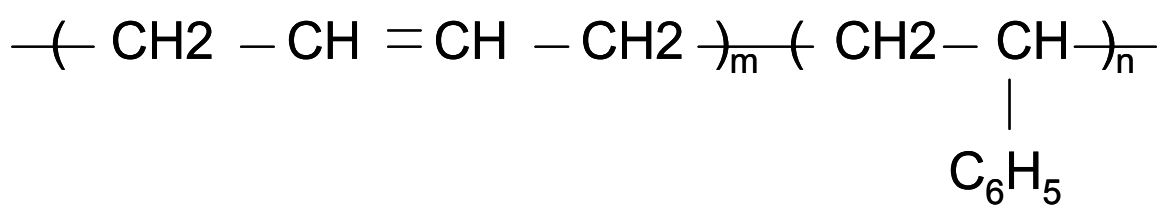

Figure 2.1: $\quad$ Monomer units of a styrene-butadiene elastomer

As a raw material, elastomer exhibits poor mechanical properties. The reason is that microscopic slippage of polymers chains occurs under deformation and leads to a macroscopic plastic behaviour (irreversible state). Consequently, various ingredients are added to practical systems in order to achieve a satisfying combination of physical properties. In particular, by mixing a raw elastomer with sulphur and bringing sufficient thermal energy, a crosslinking of the polymer matrix can occur (Figure 2.2). This process known as vulcanisation was discovered by Goodyear and results in the formation of chemical bonds networking the polymer chains. Thus, rubber becomes high elastic after crosslinking, e.g. they can undergo deformation and go back to their initial position if the force is removed. 


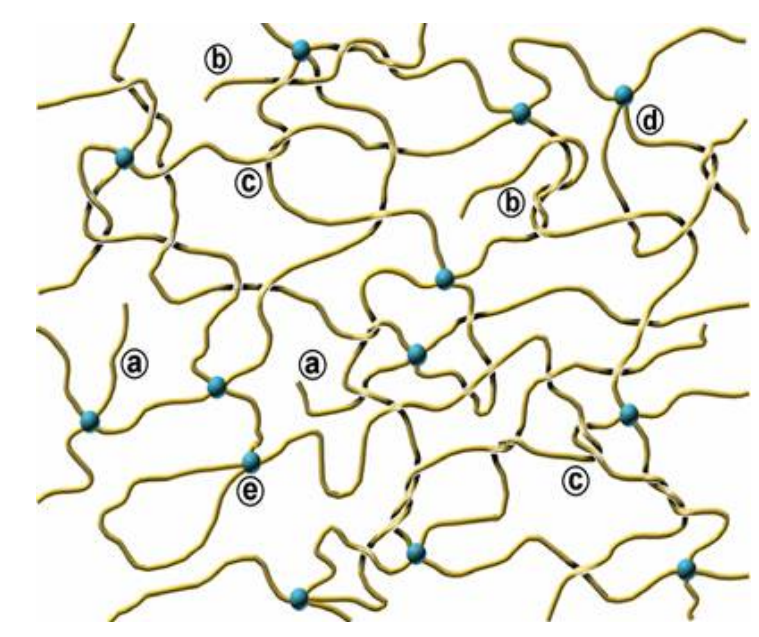

Figure 2.2: $\quad$ Elastomer network constituted by free chain ends entangled or not $(a, b)$, physical entanglements (c) and crosslinks elastically effective or not (d, e)

Because the polymer chains are long and flexible, they take up random configurations due to Brownian thermal motion. If a force is being applied, polymer chains tend to be orientated in the stretching direction but directly return to their random state when the load is released. For such materials, the elasticity is said to be entropy-based compared to enthalpy elasticity found in the case of crystalline structure observed in metals for instance. The flexible character of polymer chains can be seen on a static stress strain curve. A vulcanized rubber has the ability to undergo large deformation up to a few hundred percents while recovering its original state during unloading.

Also fillers are incorporated during the mixing process in order to enhance the mechanical properties of vulcanizates. The effect of the filler on mechanical properties can be clearly seen in Figure 2.3. At a given level of deformation, the associated stress is significantly higher for filled compounds. However, the different path for loading and unloading phase indicates that part of the mechanical energy has been dissipated. It is known as hysteresis and results from dissipative mechanisms at the interface filler/polymer matrix. 


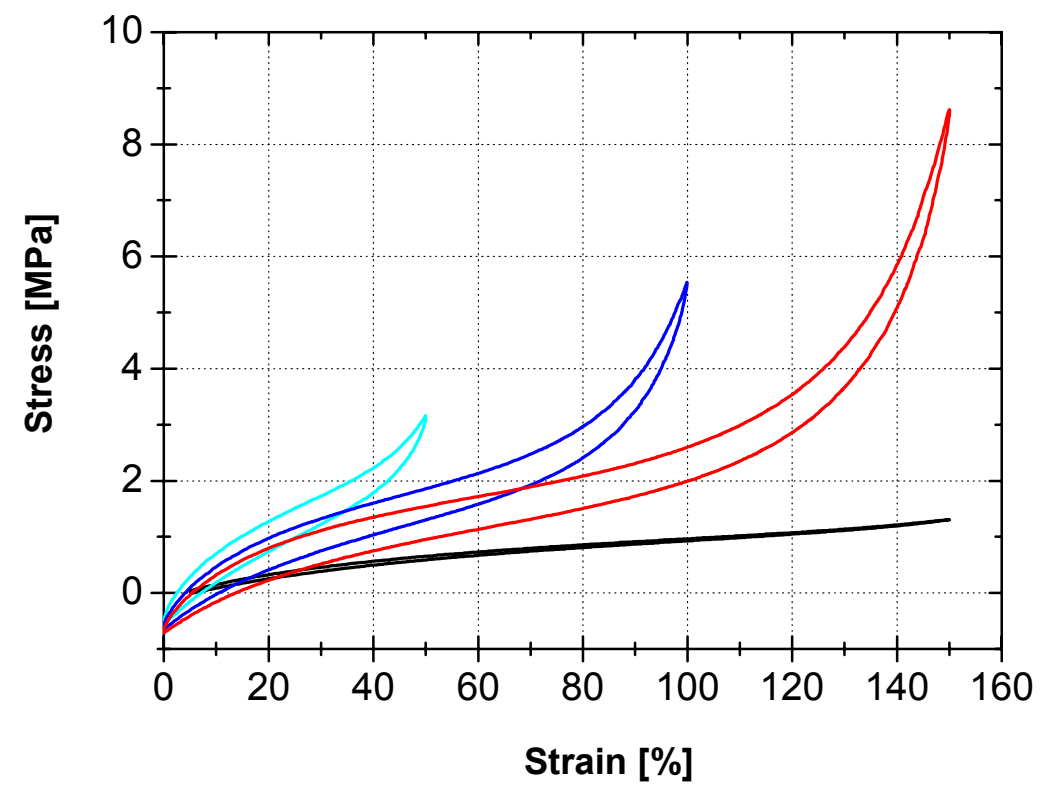

Figure 2.3: Quasi-static stress/strain curves for an unfilled (black) and filled elastomer (bright) at various strain amplitudes

\subsubsection{Linear Viscoelascity}

When a sinusoidal shear stress is imposed on a linear viscoelastic rubber, the strain will also alternate sinusoidally but will be out of phase, the strain lagging the stress. Strain $\varepsilon$ and stress $\sigma$ can be written as

$$
\varepsilon(\omega, t)=\varepsilon_{o} \sin (\omega t)
$$

and

$$
\sigma(\omega, t)=\sigma_{o} \sin (\omega t+\delta)
$$

Where $\varepsilon_{0}$ and $\sigma_{o}$ are the maximum amplitude of strain and stress, respectively; $t$ is time, and $\delta$ is the phase angle between strain and stress (Figure 2.4). At any instant in time, it is then possible to define the complex shear modulus

$$
G^{*}(\omega)=\frac{\sigma(\omega)}{\varepsilon(\omega)}=\frac{\sigma_{o}}{\varepsilon_{o}} e^{i \delta(\omega)}
$$

This quantity can be resolved into real (G') and imaginary (G') components such that 


$$
G^{*}=G^{\prime}+i G^{\prime \prime}
$$

and

$$
G^{\prime}=\frac{\sigma_{o}}{\varepsilon_{o}} \cos \delta, G^{\prime \prime}=\frac{\sigma_{o}}{\varepsilon_{o}} \sin \delta
$$

$\mathrm{G}^{\prime}$ is termed the storage modulus and is proportional to the maximum energy stored per cycle of deformation. G" is the loss modulus. The loss factor tan $\delta$ is defined by the ratio

$$
\tan \delta=\frac{G^{\prime \prime}}{G^{\prime}}
$$
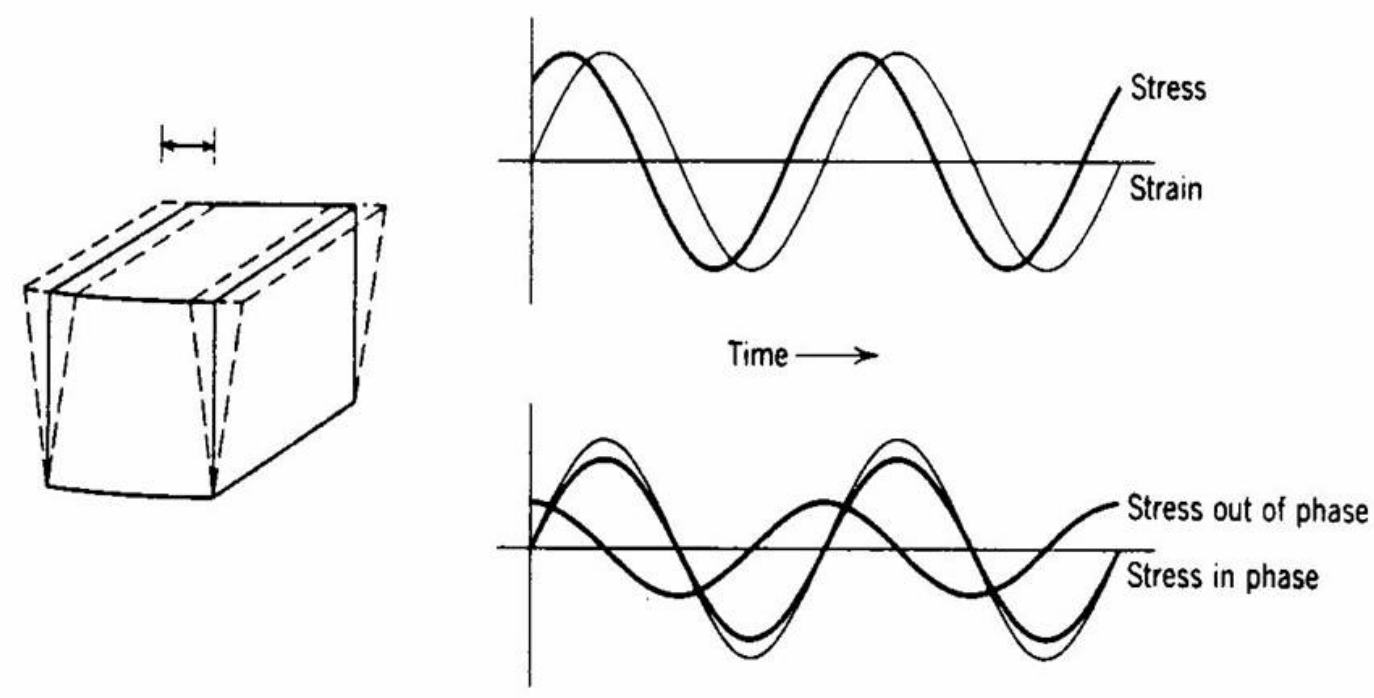

Figure 2.4: Shear deformation and dynamic response of a linear viscoelastic material

Using the general concepts of linear viscoelasticity, it is possible to investigate the temperature and frequency dependent properties of elastomers. A temperature sweep of an unfilled S-SBR is shown in Figure 2.5. The dynamic properties were measured using a Rheometrics Dynamic Spectrometer Ares operated in a torsion mode on a strip specimen (equivalent to shear) at $1 \mathrm{~Hz}$ with a strain amplitude $\varepsilon=0.5 \%$.

At sufficient low temperatures, the free volume in the polymer is so small that the movements of polymer segments and adjustments to their relative position can hardly take place within the time-scale involved in the dynamic experiment, resulting in low energy dissipation (loss factor). Under this condition, the polymer is in a glassy state with a very high elastic modulus. 
With increasing temperature molecular motions of polymer chains are energetically promoted. It follows a regime characterised by a decrease of the viscosity and high energy dissipation related to molecular adjustments scaling with the dynamic excitation of the system. In this regime, the temperature associated with the maximum of the loss modulus G" is known as dynamic glass transition temperature. Elastomers basically differ from polymers in which their glass transition temperature is located below room temperature.

Above glass transition, molecular segment can rearrange so rapidly and the viscosity is so low that the polymer solid is able to follow the imposed dynamic deformation. The material falls in the so-called rubbery region characterised by a low elastic modulus and low energy dissipation.

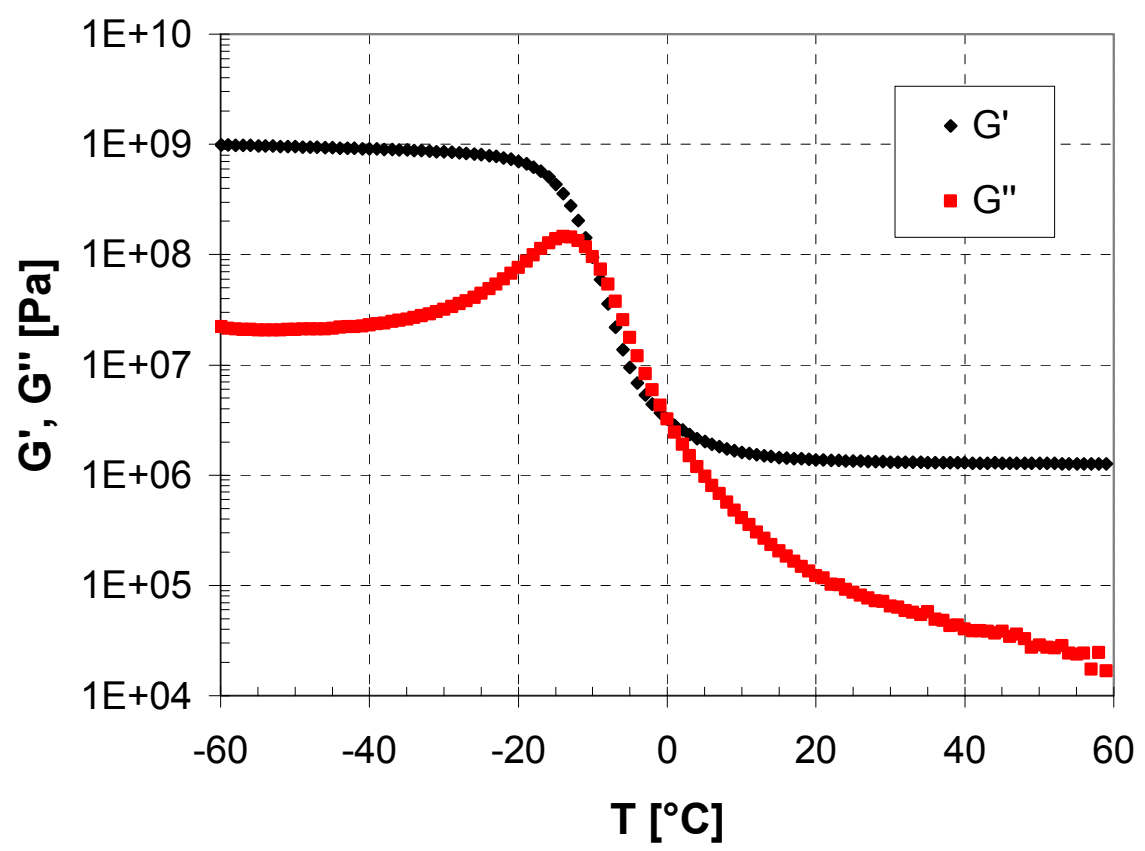

Figure 2.5: Temperature sweep for an unfilled S-SBR 5025. $\varepsilon=0.5 \%, f=1 \mathrm{~Hz}$

\subsubsection{Influence of filler}

The introduction of filler in polymer systems leads to a considerable change in dynamic properties. This affects both storage and loss moduli in a different way, implying a strong variation of the energy dissipation during dynamic deformation (loss factor). The influence of dynamic strain amplitude on the mechanical modulus has been investigated by Payne [6][7]. From his results, it could be seen that the reinforcement is only marked at low strain amplitudes. A further increase of the deformation leads to a typical non-linear behaviour generally termed as "Payne effect" and characterised by a strong decrease of the storage 
modulus G' over the strain amplitude (Figure 2.6). In contrast to G', the loss modulus G" and a fortiori the loss factor tan $\delta$ go through a maximum at a moderate strain amplitude $(5-10 \%)$ [6][7].

It has been widely accepted that the Payne effect is mainly, if not only, related to the filler network formed in the polymer matrix. Payne believed that the dissipative phenomenon experimentally observed were due to a cyclic breakdown a reaggregation of the filler network [6][7]. Following his assumption, semi-microscopical models have been derived in order to predict the non-linear dynamic behaviour of filled elastomers [62].

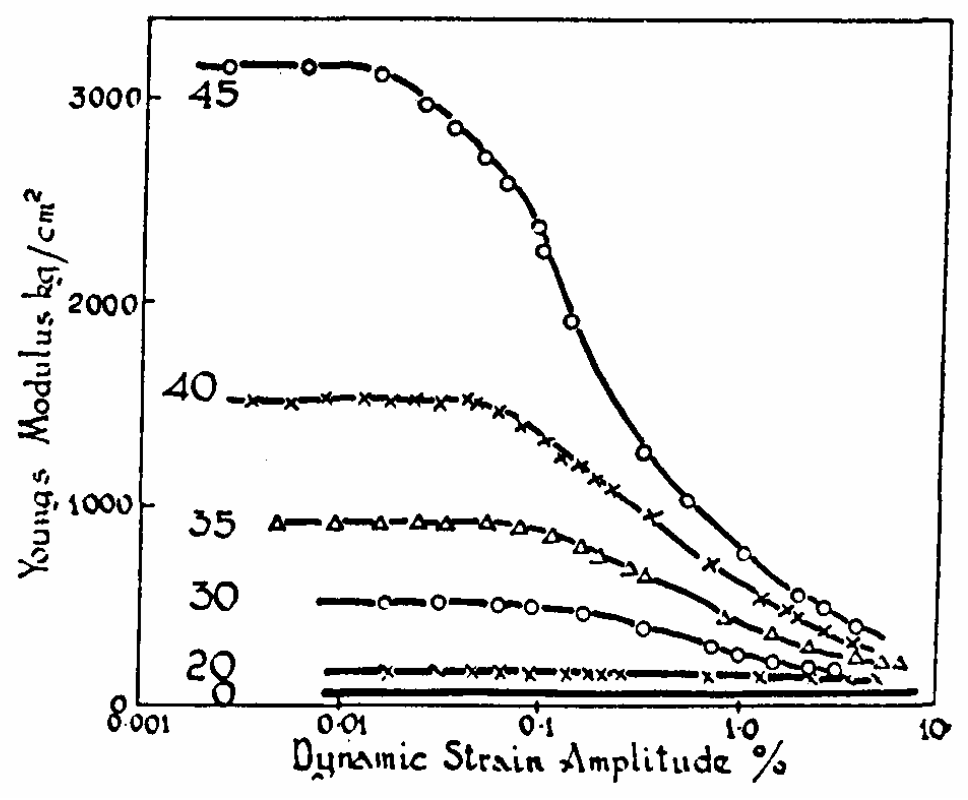

Figure 2.6: Storage modulus as a function of the dynamic strain amplitude. Numbers denote filler content in phr (from [6]).

\subsubsection{Time temperature superposition principle}

While the measurement of dynamic modulus at a given frequency can be realised over a broad range of temperatures, the same mechanical characterisation carried out at a fixed temperature by varying the frequency can applied only up to about $10^{2} \mathrm{~Hz}$. The estimation of high frequency moduli is however accessible via the time-temperature superposition principle proposed by Williams, Landel and Ferry in 1955 [8]. It states that the effect of changing the temperature is equivalent to the application of a multiplication factor $a_{T}$ on the time scale. Reducing the relaxation data to the reference temperature $T_{\text {ref }}$ leads to the following expression: 


$$
\log a_{T}=\frac{-C_{1}\left(T-T_{\text {ref }}\right)}{C_{2}+T-T_{\text {ref }}}
$$

where $C_{1}$ and $C_{2}$ are numerical parameters depending on the reference temperature $T_{\text {ref }}$. They were first thought to be universal constants for all polymers where $T_{\text {ref }}$ was set at $\mathrm{T}_{\mathrm{g}}+50^{\circ} \mathrm{C}$, with $\mathrm{T}_{\mathrm{g}}$ the glass-transition temperature of polymer. However, later studies showed that the values vary from one polymer to another [9]. Also, the WLF equation is only valid within the range of temperatures $\left[\mathrm{T}_{g} \ldots \mathrm{T}_{g}+100^{\circ} \mathrm{C}\right]$.

The applicability of the WLF equation is illustrated in Figure 2.7 for the case of an unfilled S-SBR. Since the systems must be in the thermodynamic equilibrium to be describe with the WLF formulation, sufficiently high chain mobility is required. In practice, experimental shift factors deviate from the WLF equation when the glass transition temperature of the polymer is approached.
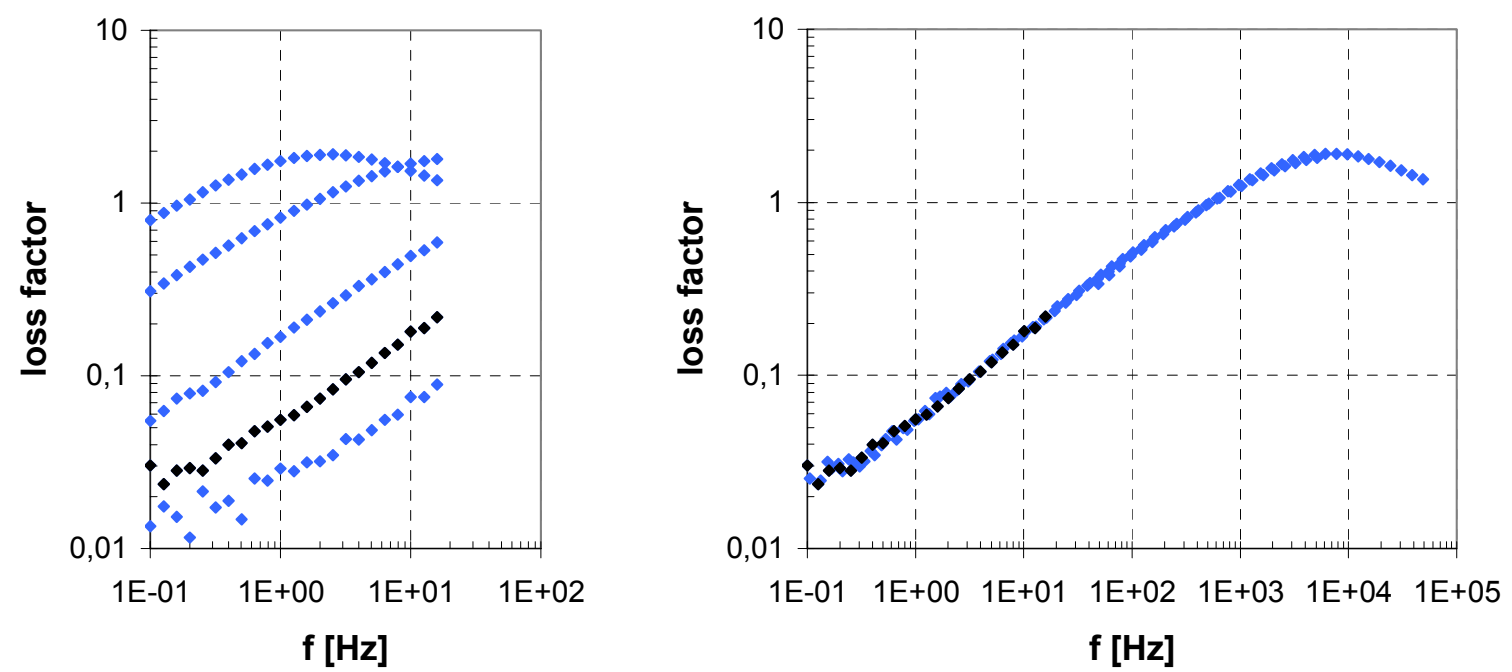

Figure 2.7: Isothermal loss factors at different temperatures (left) and horizontally shifted values (right) for an unfilled S-SBR $\left(\mathrm{T}_{\text {ref }}=20^{\circ} \mathrm{C}, \varepsilon=0.5 \%\right)$

A strictly equivalent form of the WLF equation has been originally proposed by Vogel, Fulcher and Tammann to describe the temperature dependent relaxation behaviour of glass forming liquids when approaching the glass transition regime [10][11][12]. The temperature dependent dynamic modulus $E(T)$ and typical relaxation time $\tau(T)$ are expected to follow a semi-empirical function, denoted as Vogel-Fulcher-Tamann equation (VFT), which is valid between the $T_{g}$ and $T_{g}+100^{\circ} \mathrm{C}$ and given by: 


$$
E(T)=E_{0} \exp \left(\frac{B}{T-T_{0}}\right)
$$

or

$$
\tau(T)=\tau_{0} \exp \left(\frac{B}{T-T_{0}}\right)
$$

where $\mathrm{B}, \mathrm{E}_{0}, \tau_{0}$ and $\mathrm{T}_{0}$ are adjustable parameters. Similarly to the so-called universality of WLF parameters, $T_{0}$, known as Vogel-Fulcher temperature, is found to be $T_{0} \sim T_{g}-50^{\circ} \mathrm{C}$ in limited cases. As a result, the WLF and VFT parameters are related by:

$$
\begin{gathered}
C_{2}=T_{\text {ref }}-T_{0} \\
2.303 C_{1} C_{2}=B
\end{gathered}
$$

WLF/VFT semi-empirical approaches are physically associated with cooperative molecular motions corresponding to the main relaxation process of polymer chains located above the glass transition region where thermal energy allows sufficiently high chain mobility.

The validity of WLF equation can be directly checked through dielectric spectroscopy measurements. This experimental technique is closely related to dynamic mechanical spectroscopy since some of the detected polarization mechanisms, namely those due to orientation of permanent dipoles, are in most cases related to molecular motions of molecules or segments of molecules observed under mechanical excitation. In this case, the force field is applied via the interaction between a sinusoidal electric field and bound or free charges present in the material. The nature of physical mechanisms involved during dielectric spectroscopy results in a higher sensitivity and therefore provides additional indications on local relaxation processes occurring at small length scales.

Broad-band dielectric spectroscopy covers a frequency range up to 15 decades within a wide interval of temperatures $\left[-100 \ldots+100^{\circ} \mathrm{C}\right]$ and fully characterises relaxation processes at different frequency and temperature without applying additional physical principles like the time-temperature equivalency. A comparison of both methods and the introduction of a generalized master procedure are presented in Chapter 5.

Dielectric spectroscopy consists in applying a harmonic voltage $U$ at a given amplitude and angular frequency $\omega$. The formalism derived for mechanical excitation can be applied to dielectric. It yields:

$$
U(\omega)=U_{o} \sin (\omega t)
$$


The phase angle $\delta$ and the amplitude of current $I_{0}$ are measured on the polarised material. They can be expressed as:

$$
I(\omega)=I_{o} \sin (\omega t+\delta)
$$

It is then possible to define the transfer function as:

$$
\frac{I(\omega)}{U(\omega)}=\frac{1}{R^{*}}=i \omega C^{*}, \text { with } \quad C^{*}=\varepsilon^{*}(\omega) \varepsilon_{o} \frac{A}{d}
$$

where $R^{*}$ is the complex resistivity, $C^{*}$ is the complex capacitance, $\varepsilon^{*}$ is the complex relative dielectric constant, $A$ is the specimen area and $d$ denotes the thickness of the sample.

The polarizability of the material is expressed in terms of its relative dielectric constant $\varepsilon^{*}$ which depends on both the angular frequency $\omega$ and the temperature $T$. In the same way as the dynamic modulus $\mathrm{G}^{*}$, it is decomposed into a real and imaginary part according to:

$$
\varepsilon^{*}(\omega)=\varepsilon^{\prime}(\omega)-i . \varepsilon^{\prime \prime}(\omega)
$$

In which $\varepsilon^{\prime}(\omega)$ is the real part of the complex dielectric constant associated with the polarizability of the material (storage effects). The imaginary part $\varepsilon^{\prime \prime}(\omega)$ is the dielectric loss index, a measure of dissipative mechanisms occurring during periodical excitation.

Through a wide experimental frequency window, the position and the shift of the dielectric loss maximum $\varepsilon^{\prime \prime}(\omega, T)$ can be assessed at different frequency allowing a direct characterisation of the glass transition within a broad time scale range of measurement. The temperature and frequency dependent dielectric loss of an unfilled S-SBR with $50 \%$ vinyl content is shown in Figure 2.8. The glass transition temperature, defined as the temperature at which the dielectric loss maximum $\varepsilon "{ }_{\max }$ (f) occurs, is seen to increase with increasing excitation frequency which confirms the dynamic character of the glass transition process. As a result, a relaxation time $\tau=1 / f_{\varepsilon^{\prime \prime} \max }$ can be identified for each temperature sweep at a given frequency and leads to the construction of so called retardation maps, whereby the relaxation time is plotted as a function of the inverse temperature. Accordingly, the temperature dependent relaxation behaviour is well described by a VFT semi-empirical approach (Figure 2.8 right diagram). 

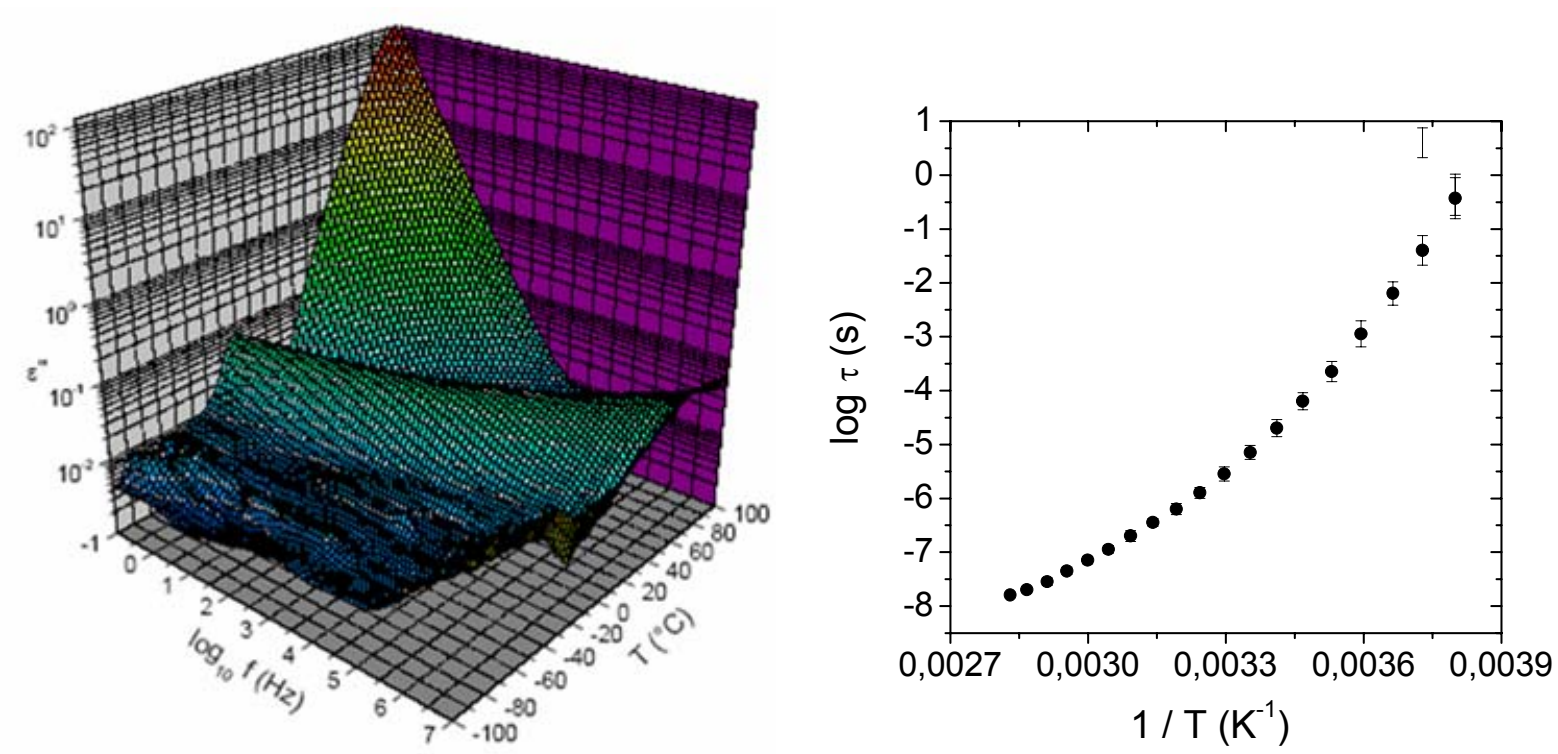

Figure 2.8: Temperature and frequency dependent dielectric loss $\varepsilon^{\prime \prime}(f, T)$ (left) and corresponding retardation map (right) for an unfilled S-SBR with $50 \%$ vinyl content.

\subsection{Friction properties of elastomers}

Friction properties of elastomers have been extensively studied for decades [1][4][13][24][48]. This section sums up the knowledge gained through pioneering experimental works combined with theoretical physical concepts. The relevance of rubber friction properties for the grip performance of tyres is finally highlighted.

\subsubsection{Physical mechanisms contributing to rubber friction}

Let us consider a rubber block sliding over a rigid substrate with a sliding velocity $v$ (Figure 2.9). The friction coefficient $\mu$ between the two solids is defined as

$$
\mu=\frac{F_{R}}{F_{N}}
$$

where $F_{R}$ denotes the friction force (tangential force) and $F_{N}$ is the normal force (load). According to this definition, the friction coefficient is expected to be independent of the specimen size. This is based on the assumption that both forces increase at the same rate, e.g. the friction coefficient is independent of the load. First experimental studies carried out by Leonardo da Vinci and Charles Augustin Coulomb on former bearing materials showed 
that the friction force is proportional to the load by a factor one quarter, which is still a realistic value for metal / metal frictional pairings.

Also, the experimental work of Coulomb showed that the friction coefficient $\mu$ is nearly independent of the sliding velocity (within a moderate range of velocity), contact area and surface roughness [1][4].

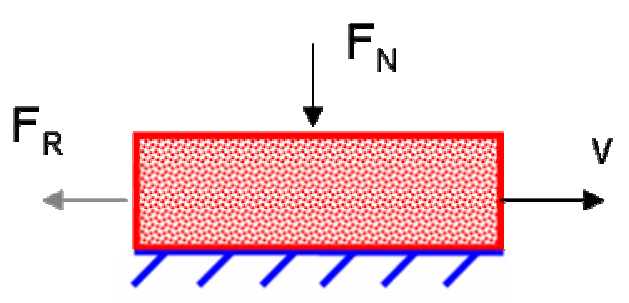

Figure 2.9: Schematic representation of sliding process

Due to the soft nature of rubber, the conclusions formulated above can not be applied for rubber sliding friction properties. The amount of contact patches is in this case strongly affected by the operating conditions and reflects the viscoelastic behaviour of rubber-like materials. As a result, the friction coefficient $\mu$ of rubbery materials depends on load, sliding velocity, temperature, surface roughness and the rubber itself.

Four physical mechanisms are usually known to contribute to rubber friction, so that the rubber friction coefficient is generally given by

$$
\mu=\mu_{H}+\mu_{A}+\mu_{C}+\mu_{V}
$$

$\mu_{\mathrm{A}}$ denotes the adhesion component and arises from molecular interactions between rubber and substrate (Figure 2.10): during the sliding process, the distribution of contact patches is constantly renewed within the contact area, inducing a cyclic formation and breakage of molecular bonds between polymer chains and surface asperities associated with dissipative effects. The adhesion component dominates under dry conditions and can be strongly reduced by lubricating the interface with specific liquids, for instance tensides.

$\mu_{H}$ is the hysteresis component and originates from macroscopic dynamic deformation of the elastomer induced by surface roughness (Figure 2.10). Since hysteresis friction is closely related to the viscoelastic spectrum of elastomers, it is expected to be influenced by a broad range of experimental parameters (sliding velocity, temperature, load, roughness) but also by the material itself. The level of hysteresis friction is promoted with increasing surface roughness and appears to be a determining factor for tyre grip performance under wet conditions. 
$\mu_{\mathrm{C}}$ is the so-called cohesion component that describes wear processes at the interface. It is expected to occur when using substrates with sharp asperities, like corundum surfaces.

Finally $\mu_{\vee}$ is denoted as viscous component. The presence of lubricant at the interface gives an additional contribution on the resulting friction force whose amplitude depends on the viscosity of the fluid and the nature of contact. Practically, for short contact times, e.g. high sliding velocities, the viscosity behaviour of water requires high shearing forces to be drained out of the contact area so that a fluid thickness may subsist at the interface and thereby limit the generation of contact stresses (elasto-hydrodynamic) [5].

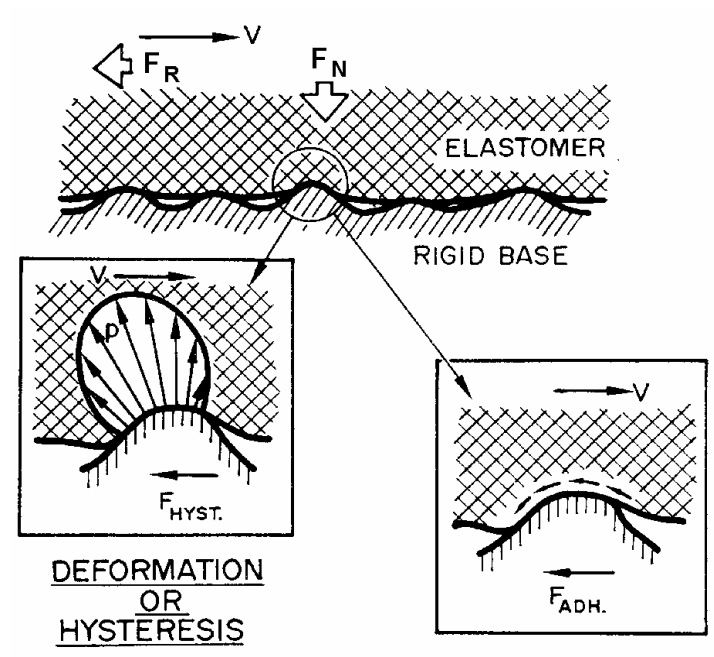

ADHESION

Figure 2.10: Schematic representation of the main mechanisms contributing to rubber friction (from [1])

The experimental conditions under which the existence of each component is promoted have been widely discussed in the literature [1][17][32]. The deformation component mainly plays a role on rough surfaces where the size of the asperities is sufficiently large to locally deform the rubber. On dry and smooth surfaces, the friction coefficient is mainly due to the adhesion component. The contribution of abrasion should be considered on rough surfaces with sharp asperities, for instance silicon carbide paper, and under severe experimental conditions (high sliding velocity combined with high load). In the next section, pioneering experimental studies give a deeper insight on the physical mechanisms involved during rubber friction on different surfaces as well as their relation to viscoelastic properties.

\subsubsection{Rubber friction on smooth surfaces}

The effect of temperature and sliding velocity on dry friction between a roughened rubber pad and a wavy glass surface has been investigated by Grosch [13]. He showed that the 
measured friction coefficient could be shifted along the velocity axis for a given reference temperature in the same way as viscoelastic moduli. The temperature dependence of the obtained shift factors was found to follow the WLF equation gained from relaxation spectroscopy. As a result, the isothermal friction coefficient could be assembled in a unique master curve covering a wide range of sliding velocity (Figure 2.11). It has to be noted that the measuring range of sliding velocity was chosen up to maximum $1 \mathrm{~cm} / \mathrm{s}$ in order to avoid thermal effects occurring within the contact area.
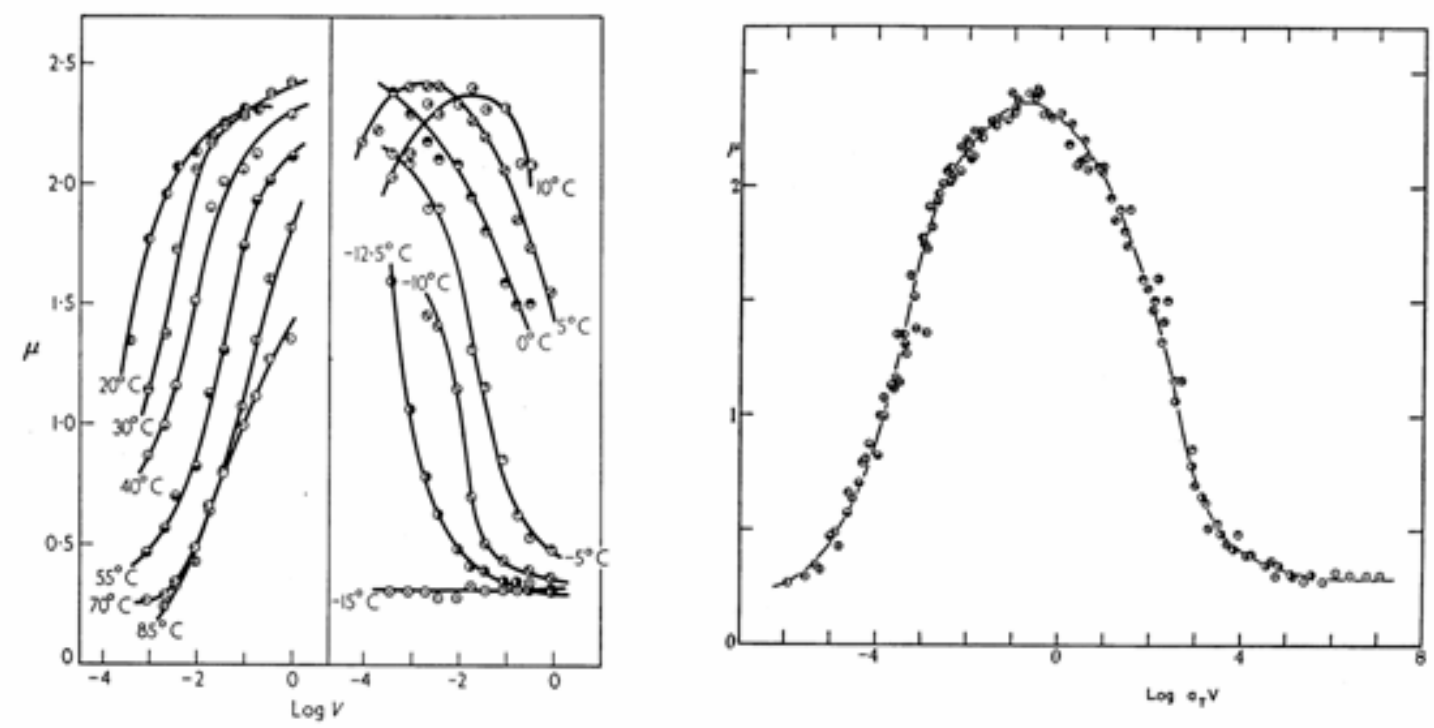

Figure 2.11: Isothermal friction curve (left) and master curve (right) of a NBR compound on dry wavy glass (from [13])

The applicability of WLF equation was a strong evidence for the viscoelastic nature of dry friction processes of rubber on smooth surfaces. Further experiments carried out for other polymers showed a similar bell-shaped friction curve going through an adhesion peak. The velocity at which the friction maximum occurs could be related with the maximum of the loss modulus E" found in mechanical spectroscopy (Figure 2.12). These two quantities could be related to one another by a molecular length scale as following:

$$
v_{\max }(\mu)=\lambda f_{\max }\left(E^{\prime \prime}\right)
$$

where $\lambda=6 \mathrm{~nm}$.

This was a milestone for the development of analytical models describing the rubber friction on smooth surfaces. Physically, adhesion friction is considered as a stationary stochastic process consisting in the formation and breakage of linking chains which bind the polymer body to the surface [28][29]. The molecular mechanism of breaking off is assumed to be 
either due to thermal excitation or the stress reached at maximal elongation. Consideration of detailed molecular aspects leads to an extended formulation of the adhesive friction on smooth surfaces [33][34].

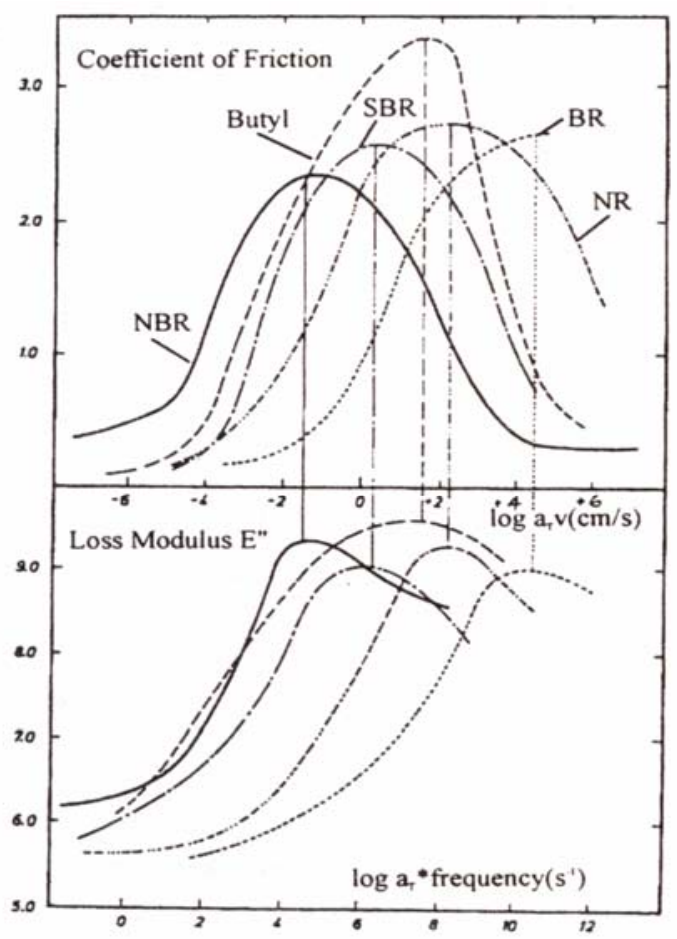

Figure 2.12: Correlation between dry friction master curves (NBR on Glass) and loss modulus (from [13])

Friction measurements carried out on individual glass lenses and a smooth rubber surface differ from the Grosch results and exhibit a slight variation of the friction coefficient over the whole range of velocity [20]. By increasing the roughness of the rubber surface, the friction coefficient appeared to be more rate-sensitive and followed a similar path as the classical Grosch data. This makes clear that an additional factor hinders the increase of friction for smooth surfaces.

Optical observations of the contact between a rubber sphere and a plate glass during sliding friction revealed the existence of wrinkles (Figure 2.13). They have been described by Schallamach as wave of detachment and travel in the direction in which the rubber moves relative to the glass [32]. In this case, no true sliding occurs in the contact area, but the friction originates from the peeling and unpeeling phenomenon on both sides of the fold. As a result, the increase of friction coefficient is limited within the range of occurrence of Schallamach waves (Figure 2.13). A roughening of the rubber surface hinders the appearance of waves of detachment and suggests that they are driven by a stress-relieving mechanism that limits the build-up of friction with increasing sliding velocity. 


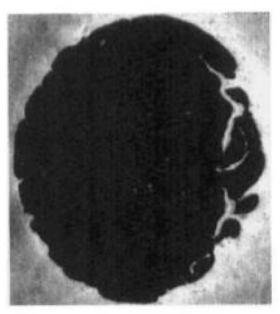

(a)

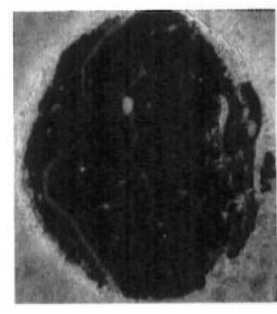

(b)

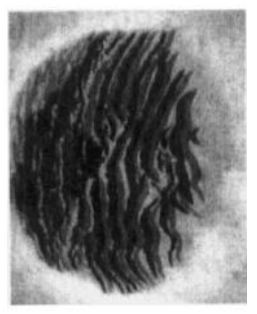

(C)

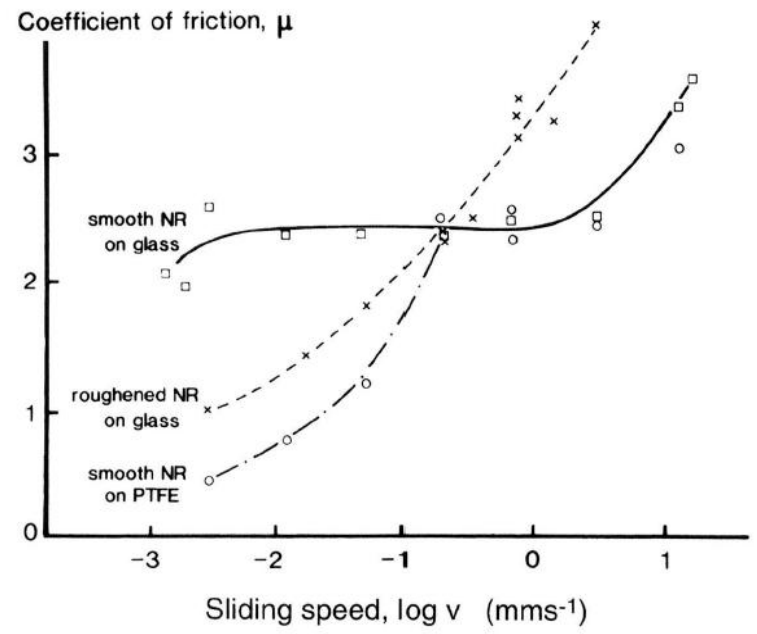

Figure 2.13: Friction coefficient as a function of the speed for a smooth and roughened rubber against glass and PTFE. The insert shows the appearance of : (a) bursts of wavelets at $0.4 \mu \mathrm{m} / \mathrm{s}$, (b) regular Schallamach waves at $0.4 \mathrm{~mm} / \mathrm{s}$, (c) ridges at $6 \mathrm{~mm} / \mathrm{s}$ (from [24])

\subsubsection{Rubber friction on rough surfaces}

A surface associated with a roughness parameter $R_{q}$ - corresponding to the statistical standard deviation of profile - higher than a few micrometers is generally said to be rough. It includes a wide range of surfaces used in industrial applications, from sandblasted steel surfaces until road tracks (asphalt, concrete). Roughness has two opposite effects on rubber friction: first, through the micro-indentation of surfaces asperities into the elastomer, a part of the mechanical energy is dissipated through viscoelastic losses (hysteresis friction). On the other side, the reduction of the contact area with increasing roughness should decrease the level of adhesion.

Based on the same experimental principle presented in the former section, velocity dependent friction curves were measured at different temperatures within the range of low sliding velocity on a dry silicon carbide surface [13]. Results show that the friction values can 
be assembled according to a master curve technique, which underlines the viscoelastic nature of rubber friction on rough surfaces (Figure 2.14). Compared to results on smooth surface, the main friction peak is located at higher sliding speeds and correlates with the position of the loss factor maximum. By using Equation (2.18), a length scale can be calculated and is found to be nearly equal to the average separation of surface grains. This clearly reflects the influence of the roughness and the nature of hysteresis friction.

Beside the friction maximum associated with the hysteresis component, a second peak is found at a sliding speed roughly equal to the one found on glass and therefore interpreted as adhesion peak $\left(\mathrm{v} \sim 10^{-2} \mathrm{~cm} / \mathrm{s}\right)$. Indeed, if the silicon carbide substrate is dusted with magnesium powder, the contact rubber/silicon carbide is replaced by the adhesion to magnesia while the low sliding velocity peak is found to vanish (Figure 2.14). This demonstrates the existence of both mechanisms on rough surfaces, whereby the level of adhesion appears to strongly depend on the contact conditions.

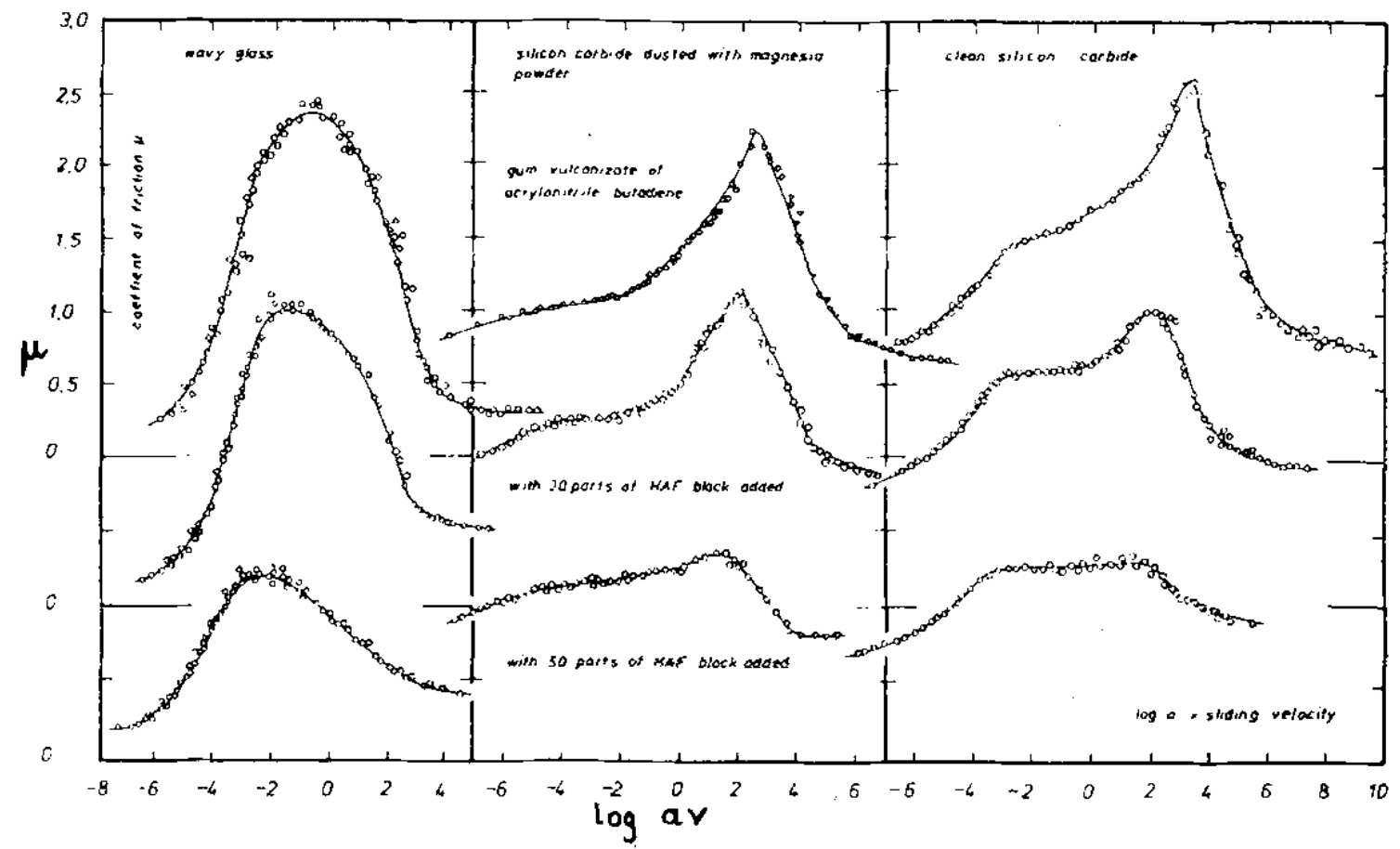

Figure 2.14: Friction master curves for a NBR compound on wavy glass (left), silicon carbide dusted with magnesium powder (middle), clean silicon carbide (right) with different filler content: $0 \mathrm{phr}$ (top), $30 \mathrm{phr}$ (middle) and $50 \mathrm{phr}$ (bottom) (from [13])

The incorporation of filler has two effects on the friction properties: first, the maximum in friction is strongly decreased, independently of the roughness amplitude. Also, the friction coefficient tends to reach a saturation regime with increasing filler content and exhibits a 
plateau value over many decades (Figure 2.14). This is mainly due to a pronounced decrease of the hysteresis friction maximum at high sliding velocity. It is interesting to note that a similar decrease occurs for the frequency dependent loss factor tan $\delta$ determined by relaxation spectroscopy measurements. The saturation of dry friction feature has been confirmed later by Rieger for carbon black filled compounds measured on emery paper [16]. In addition, the velocity at which the dry friction is saturating appears to be directly connected with the glass transition temperature of the elastomer.

\subsubsection{Effects of lubricants}

The presence of lubricant at the interface can prevent the rubber from coming into intimate contact with the counter surface. This is especially true if the sliding velocity is very low or if the viscosity of the lubricant is very high. The conditions of appearance of such films have been expressed in the frame of the theory of hydrodynamic lubrication [48]. If the load is increased, the lubricant film, through enhanced viscosity, is even less likely to be drained out of the contact area and the related theory is known as elastohydrodynamic lubrication $(E H L)$. By using optical interferometry, the thickness of the lubricant film has been measured during dynamic contact between a rubber hemisphere and a glass plate [26]. Also, in presence of water, the high level of the friction coefficient at very low sliding speeds indicates a film breakdown. There is a general tendency towards film breakdown at low sliding velocity, but a notable exception occurs if water contains a small amount of soap. In this case, the friction values are found to be very low which suggests that the soap film is stabilized and intimate contact is hindered at the interface [26].

An experimental procedure has been proposed to separate the adhesion and the hysteresis component [17]. By using a modified British Pendulum Tester, rubber tread compounds were tested against steel sliders with various radii at a sliding speed $v \sim 3 \mathrm{~m} / \mathrm{s}$ at different temperatures. The rubber plate was wetted with water and then covered with a thin oil coated polyethylene foil (Figure 2.15). In this way, the deformation process was not affected at all while the low viscosity oil film was assumed to completely eliminate the adhesion component.

The results show that adhesion mainly contributes to the frictional process over the whole range of temperatures. As expected, both components exhibit an opposite dependence with respect to the temperature: indeed, at high temperatures, the rubber becomes softer and generates a higher contact area, e.g. more potential adhesion. At the same time, the decrease of the loss modulus leads to lower energy dissipation, e.g. less hysteresis friction. 


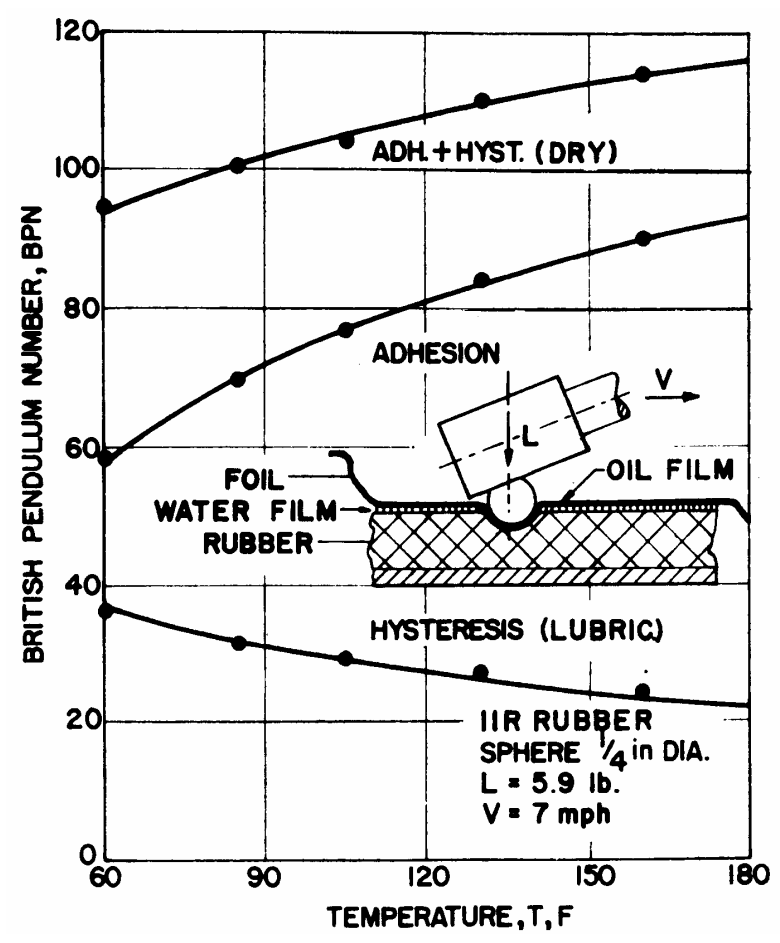

Figure 2.15: Separation of adhesion and hysteresis friction component by lubricated foil technique (from [17])

The nature of contact on rough surfaces makes the evaluation of the lubricant thickness more complicated. Indeed, contact patches are statistically distributed over the interface and the roughness induces a highly heterogeneous pressure field within the contact area with a constantly renewed population of contact patches during the sliding process. However, as observed on smooth surfaces, the presence of a water film decreases the level of friction. The efficiency of surface wetting strongly partially depends on the surface topography, e.g. the asperity sharpness. Silicon carbide surfaces show a minor difference between dry and wet friction which indicates that local pressures are high enough to break the water film at the interface and form dry islands within the contact area. Also, the addition of detergent appears to stabilize the lubricant film since a decrease of the wet friction coefficient is observed over the whole range of sliding speeds [14][70]. Furthermore, the hump - interpreted as adhesion peak - experimentally found on silicon carbide wetted with distilled water around $v \sim 1 \mathrm{~cm} / \mathrm{s}$ simply vanishes if a low amount of detergent is added to the water solution (Figure 2.16). This confirms the assumption that the adhesion component can be significant on rough sharp surfaces even if the surface is fully wetted. Detergents are known to stabilize the interfacial layer between elastomers and rough substrates, thus hindering the occurrence of adhesion effects. Consequently, if abrasion can be neglected and the soap concentration does not significantly increase the viscosity of lubricant, friction results under moderate wet contact conditions with detergent can be solely identified with hysteresis friction. This remark has 
strong implications on the experimental procedure used to separate adhesion from hysteresis friction of elastomers on rough surfaces.

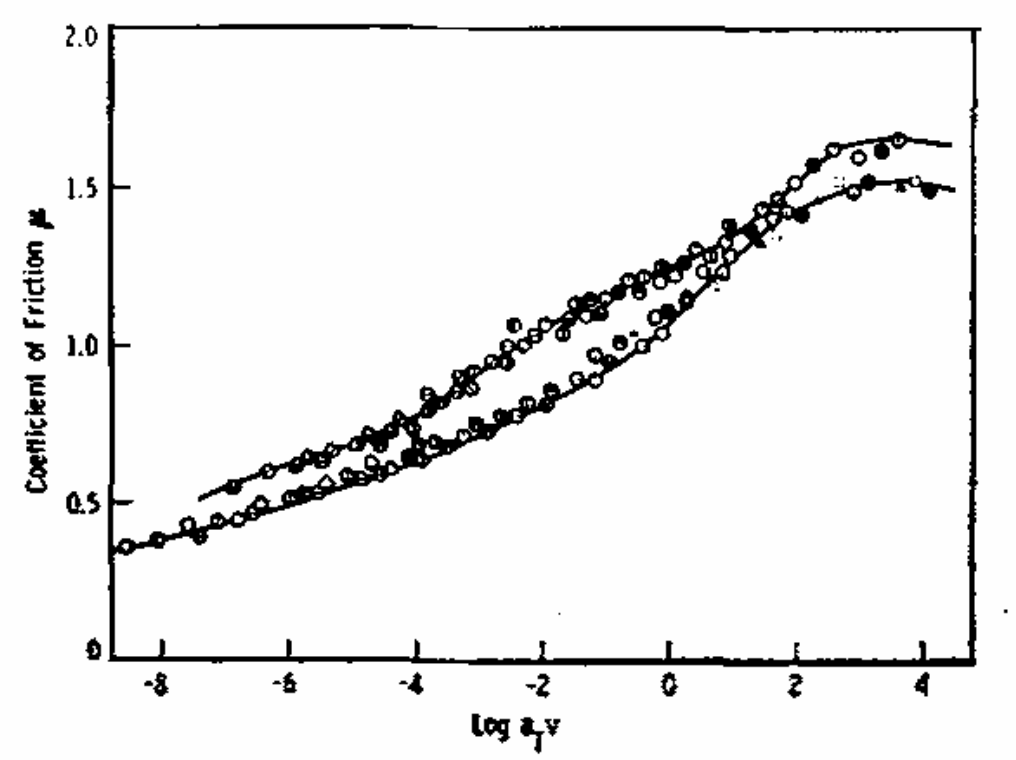

Figure 2.16: Friction master curve for a filled NBR on silicon carbide wetted with distilled water (upper curve), distilled water $+2 \%$ detergent (lower curve) (from [14])

\subsection{Relation to tyre performance}

The comprehension of physical mechanisms involved during rubber friction on rough surfaces is a decisive step for the prediction and the improvement of tyre wet grip performance. Combined with abrasion and rolling resistance, the evaluation of key-properties is generally represented in the so-called "magic triangle" of tyre performance. Thereby, the impact of tread formulation on tyre performance can be easily estimated compared to a reference compound. The simultaneous improvement of tyre key-properties still remains a challenging task for material developers. A global increase of material performance can be achieved by a fine tuning of components, as illustrated by the introduction of "green tyres" in the 90 s based on the replacement of carbon black by highly dispersible precipitated silica fillers which led to optimized wet grip and rolling resistance properties [5].

Let us consider a tyre submitted to a load $F_{N}$ and a tangential force $F_{X}$ in the sliding direction. During acceleration and braking phases, the velocity of the wheel differs from the vehicle speed and one can define a slip as following: 


$$
s_{l}=\frac{v_{r}-v_{0}}{v_{0}} \quad[\%]
$$

Where $v_{r}$ is the rotational velocity of the wheel and $v_{0}$ is the rotational velocity without braking or accelerating torque (vehicle). For instance, during braking, $s_{I}=1$ means that the vehicle wheels are completely blocked. From these considerations, the level of longitudinal performance characterised by the friction coefficient $\mu\left(s_{1}\right)=F_{X} / F_{N}$ can be represented on a unique curve (Figure 2.17). The level and the position of the $\mu\left(s_{I}\right)$ maximum depend on the type of rubber, road texture, and operating conditions (temperature, vehicle velocity). Under given conditions, the friction maximum $\mu_{\max }$ corresponds to the optimum that can be achieved during braking process. The following decrease at high slip rates displays the negative impact of temperature on wet grip performance since this effect is amplified at higher starting vehicle velocity.

In a similar way, a side-force coefficient can be defined when the tyre makes an angle with the trajectory of the vehicle. A $\mu$-slip curve can be assessed for an estimation of the tyre performance during cornering.

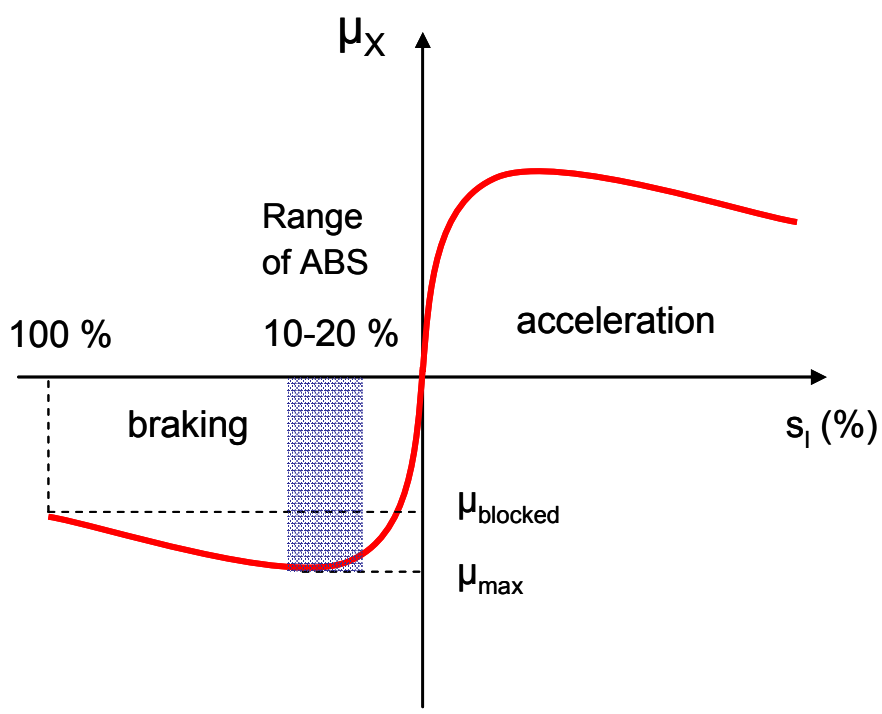

Figure 2.17: Typical $\mu$-slip curves for longitudinal accelerating and braking phases. The ABS range is indicated in the braking region

With the introduction of ABS-Braking system, locked wheel becomes impossible. The braking torque is constantly adjusted in the range of slip associated with the maximal adherence $\left(s_{1} \sim 10 \%\right)$. The work of Roth gave some precisions on the mechanisms involved during ABS braking phases [81]: in the entrance zone of the contact, the rubber is first compressed and then brought into shear through the velocity of the vehicle (deformation regime). If the potential of adherence of the rubber is reached, the tread element starts to slide and friction takes place at the interface until the exit region (slip regime). The deformation regime is 
associated with sliding velocities around $v \sim 1 \mathrm{~cm} / \mathrm{s}$ which gradually increase in the slip regime up to $v \sim 1-3 \mathrm{~m} / \mathrm{s}$.

The level of skid resistance is determined via the ability of the material to generate tangential stresses in the contact area. On dry rough surfaces, the energy dissipation arising from local deformation of the rubber and interfacial effects within the area of contact mainly contribute to the friction coefficient. With the presence of lubricants, adhesion might be affected under certain conditions but local contact pressures are generally high enough to break the water film and build dry contact islands at moderate sliding speeds [5][80].

The granularity of road surfaces plays a major role on rubber deformation during sliding processes. As shown in Figure 2.18, the level of macrotexture - from fine to coarse - controls the rate at which friction decreases at high sliding velocities under wet conditions by promoting the drainage ability of the surface and therefore reducing the risk of aquaplaning.

On the other side, the type of microtexture - polished, rough - appears to control the level of friction, e.g. asperities at small length scales offer additional contact possibilities during dynamic contact. Physically, local high pressures due to micro-roughness are likely to cause the breakage of the interfacial water film and the formation of dry contact islands which finally increases the level of friction. Hence, while the influence of micro-asperities is marginal under dry conditions, it considerably improves the skid resistance under wet conditions.
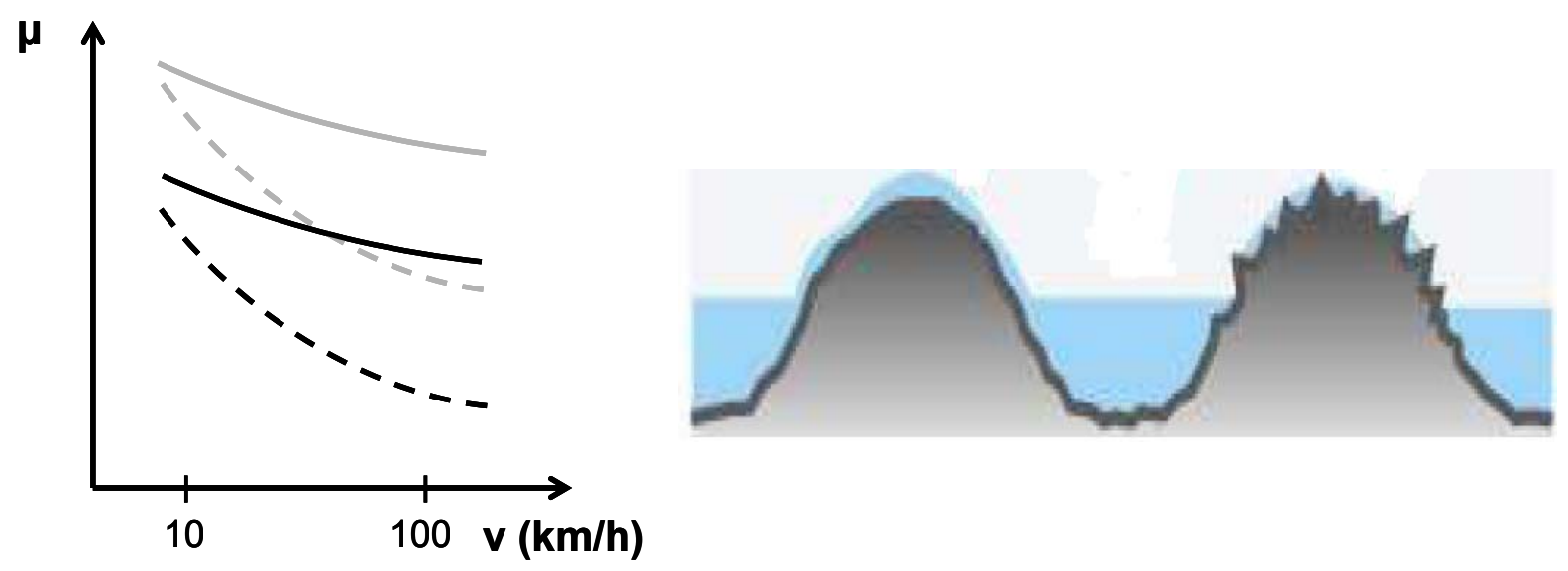

Figure 2.18: Schematic $\mu(v)$ curve under wet conditions with coarse (full) and fine (dashed) macrotexture, rough (grey) and polished (black) microtexture (left diagram). Right diagram: Asperities with and without microtexture (from [5])

Since the sliding process can be seen as a multi-scale problem with a dynamic deformation of rubber by surface asperities, the level of friction is subsequently controlled by the viscoelastic response of the material within a defined range of excitation frequencies. In the 
high frequency region, rubber undergoes a dynamic glass transition characterised by maximal internal losses and therefore the highest dissipative potential for friction properties. This is why the wet skid behaviour is often correlated with the glass transition temperature or viscoelastic quantities in the high frequency region [57][58][82]. Wet grip predictors might lead to satisfying correlation but they principally do not reflect the complexity of dynamic contact between rubbery materials and rough substrates.

A decisive step for the modelling of rubber friction was achieved through the introduction of fractal concepts for the description of roughness. Self-affinity describes a statistical invariance of the surface morphology under anisotropic spatial dilations and was shown to encompass a wide variety of natural and manufactured surfaces, including rough and road surfaces [46]. The associated mathematical treatment of self-affinity is presented in the next section. The fractal character of rough surfaces allows a length-scale dependent characterisation of morphology and implies a multi-frequency excitation of elastomers during the sliding process (Figure 2.19).

Based on this fact, models have been derived to predict rubber sliding friction on rough surfaces arising from the hysteretically lost energy during dynamic contact, namely hysteresis friction [59][60][63][48][49]. The friction coefficient is expressed in terms of a friction integral reflecting the spectrum of roughness involved during the deformation process. A recent work showed a good agreement between predictions of hysteresis friction and measurements performed on a rough lubricated surface [71].

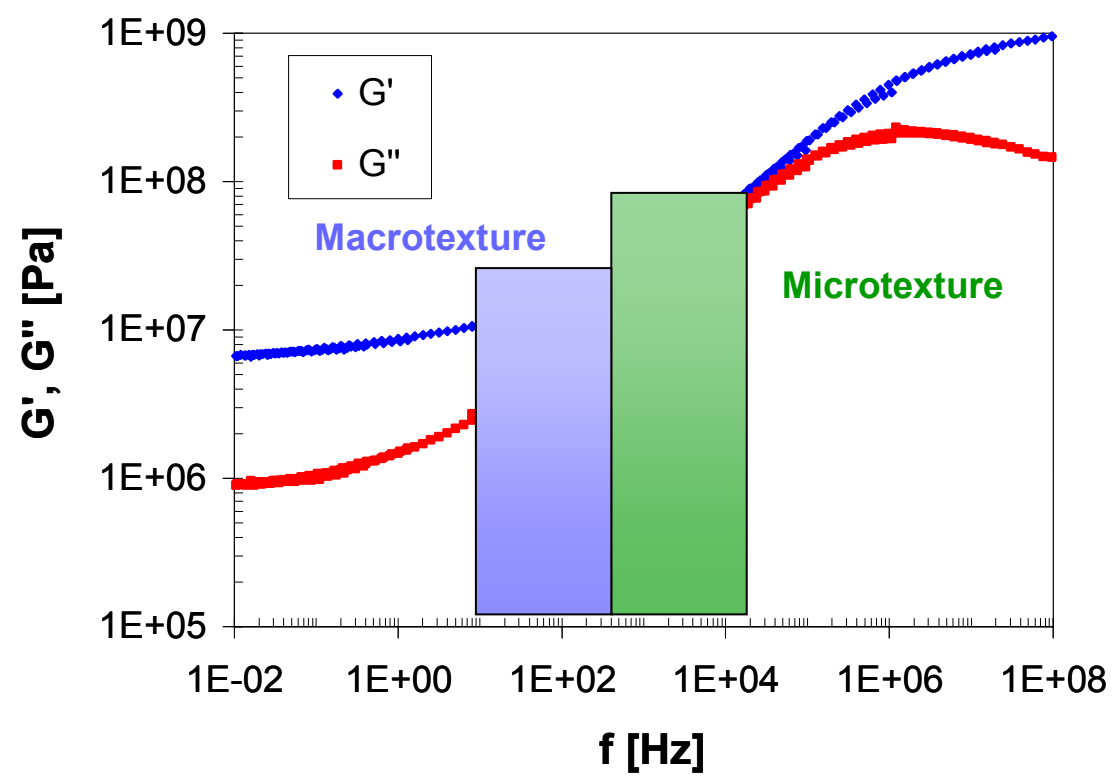

Figure 2.19: Schematic representation of excitation frequencies during sliding process with separate contributions of micro- and macrotexture 


\section{Modelling of rubber friction on rough surfaces}

This chapter presents a novel theoretical approach for the prediction of rubber friction on rough surfaces. After a brief introduction of the Hertz theory, simplifications are derived in the case of the contact between rubber and a rigid asperity. The modelling of contact is treated within the frame of an extended Greenwood-Williamson theory allowing an analytical formulation of contact parameters as a function of material quantities and operating conditions. Compared to earlier friction theories, a major step is achieved by considering the self-affinity of rough surfaces, whereby roughness is characterised through its morphological invariance over many length scales. As a result, based on a multi-scale description of contact, hysteresis and adhesion friction are derived for elastomers on rough substrates.

\subsection{Modelling of non-sliding contact}

When two solids are brought into contact, the area over which they touch determines the ability to generate shear stresses during the sliding process. Basic concepts of the Hertz theory are detailed in this section for the contact of two elastic solids. Moreover, rubber specific effects are presented regarding the formation and breakage of contact patches on rough substrates in the non-sliding mode.

\subsubsection{Theory of Hertz}

An analytical formulation of stresses at the contact point of two elastic solids was already derived by Hertz [38]. Let us consider two solids with respective radii $R_{1}$ and $R_{2}$, filled with uniform isotropic linearly elastic media characterised by Young's moduli $E_{1}, E_{2}$ and Poisson ratios $v_{1}, v_{2}$. Suppose that the solids are compressed with a normal force $F_{N}$, the contact will then change from a point into a finite area characterised by a contact radius while the deformed solids approaches each other from a distance $\mathrm{h}$ given by (Figure 3.1):

$$
h=F_{N}{ }^{2 / 3} D^{2 / 3}\left(\frac{1}{R_{1}}+\frac{1}{R_{2}}\right)^{1 / 3}
$$


By introducing an effective Young modulus as following:

$$
D=\frac{3}{4}\left(\frac{1-v_{1}^{2}}{E_{1}}+\frac{1-v_{2}^{2}}{E_{2}}\right)
$$

The contact radius for two spherical elastic solids brought into contact is given by:

$$
a=F_{N}^{1 / 3} D^{1 / 3}\left(\frac{R_{1} R_{2}}{R_{1}+R_{2}}\right)^{1 / 3}
$$

In this particular case, the solids penetration $\mathrm{h}$ displays the difference between the sum $R_{1}+R_{2}$ and the spheres center-to-center distance which is given by:

$$
h=\left(F_{N} D\right)^{2 / 3} R_{1}^{-1 / 3}
$$

and

$$
a=\left(F_{N} D R_{1}\right)^{1 / 3}
$$

It can be deduced that:

$$
a^{2}=h R_{1}
$$

The contact area is then given by:

$$
A=\pi a^{2}=\pi\left(F_{N} D R_{1}\right)^{2 / 3}=\pi R_{1} h
$$

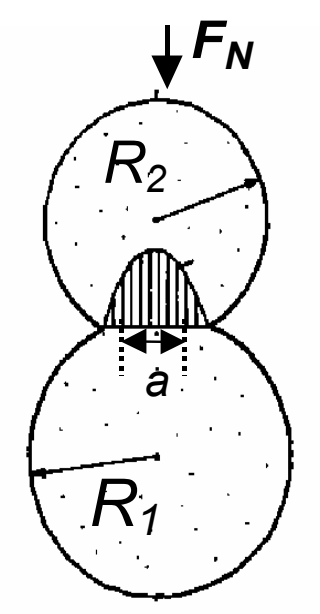

Figure 3.1: $\quad$ Contact between two spheres with radii $\mathbf{R}_{\mathbf{1}}$ und $\mathbf{R}_{\mathbf{2}}$ 
Following the Hertz theory and by assuming that the tangential friction force is proportional to the contact area derived in Equation (3.7), the friction coefficient is found to vary with $\mu \sim F_{N}^{-1 / 3}$, e.g. the friction coefficient decreases with increasing load.

\subsubsection{Consideration of interfacial effects}

Let us consider the contact between a blunt asperity and a smooth rubber surface. Through its low modulus, the rubber is able to locally highly deform so that intimate contact is formed between both solids and molecular attractions (Van der Waals) are likely to take place at the interface. As a consequence, this effect modifies the apparent load in the contact area and one should expect an increase of the contact radius.

In spite of earlier works in this field of research [39][40], the solution of the adhesive contact between rigid sphere and rubber-like materials has been completely formulated by Johnson, Kendall and Roberts [35]. Based on an energy balance theory, a generalized expression of the contact radius $a$ is derived and given by

$$
a^{3}=\frac{9 R}{16 E}\left(F_{N}+3 \pi \gamma R+\left(6 \pi \gamma R F_{N}+(3 \pi \gamma R)^{2}\right)^{1 / 2}\right)
$$

where $F_{N}$ is the applied load, $R$ the radius of the rigid sphere, $E$ is the Young elastic modulus of the rubber, $\gamma$ is the thermodynamic work of adhesion given by the Dupré equation:

$$
\gamma=\gamma_{1}+\gamma_{2}-\gamma_{12}
$$

With $\gamma_{1}$ and $\gamma_{2}$ the respective surface energies and $\gamma_{12}$ an interaction term. If no attraction occurs between the two solids $(\gamma=0)$, the classical Hertz contact formulation is found again. Also, if the load vanishes, the contact area tends to a finite value due to the interfacial term.

\subsubsection{Kinetics of adherence}

It has been seen that the adhesion is responsible for an increase of the contact radius when a blunt asperity is brought into contact with a smooth rubber surface. Also, molecular attractions give an additional contribution on the pull-off force during the separation of both solids. The amplitude of the adhesion force is in this case strongly dependent on the rate of separation, e.g. the peeling velocity. Indeed, as the load is removed, a peeling mechanism of 
the rubber is observed at the contact periphery which suggests that the pull-off force is driven by the local viscoelastic behaviour of rubber associated with high strains. Peeling-off energies might vary with a factor hundred when the rate of separation is increased. Investigations of the peeling energy showed that it depends on the equilibrium value, the rubber type and the peeling rate [19].

Finally, drop tests of a steel ball on a rubber plate confirmed the viscoelastic character of adhesion that was found to strongly depend on the impact velocity, e.g. the rate of separation or breakage of contact patches [25].

\subsubsection{Impact of roughness}

Increasing the surface roughness leads to a reduction of the contact area with an elastomer specimen. In this case, the contact can be decomposed in distinct islands and represents only a small fraction of the nominal contact area. Consequently, the strength of the adhesion is expected to decrease. Previous experimental investigations have been carried out at low rates of separation $(v<1 \mathrm{~mm} / \mathrm{s})$ and showed that a roughness in the order of $1 \mu \mathrm{m}$ was sufficient to reduce the adhesion to a low level [27]. This effect was furthermore amplified by increasing the rubber stiffness.

However, recent works focused on the adherence force between rubber and road surfaces during hastily separation [36][37]. It could be seen that the recorded pull-off force runs through a negative value during the separation process, suggesting the existence of attraction forces, namely adhesion. The rate dependence was also found to partially fulfil formulated assumptions considering the kinetics of adherence [21].

\subsection{Contact mechanics on rough surfaces}

The frictionless contact between rough elastic solids is described next based on the pioneering contribution of Greenwood and Williamson. Following this, an extended modelling of the dynamic contact between rubber and rough surfaces during sliding friction can be derived, whereby an analytical approach is proposed for the evaluation of the summit height distribution required to determine contact parameters.

\subsubsection{Theory of Greenwood-Williamson}

A formulation of the elastic contact between rough and smooth surfaces has been proposed by Greenwood and Williamson [41]. Following the views from Hertz, the rough surface is 
approximated by spheres having the same radius $\mathrm{R}$. However, the spheres are randomly distributed over a mean plane and exhibit a Gaussian height distribution $\phi(z)$ (Figure 3.2). Then, the probability to find an asperity between the interval $[z, z+d z]$ is given by $\phi(z) d z$. If one denotes as $d$ the distance between the mean plane of the rough substrate and the smooth surface, the number of summits in contact can be calculated as following:

$$
n=N \int_{d}^{\infty} \phi_{s}(z) d z
$$

where $\mathrm{N}$ is the total number of summits.

The resulting total force is then the sum of all contact forces acting on single asperities and is given by:

$$
F_{N}=\sum_{i} F_{n, i}=N D^{-1} R^{1 / 2} \int_{d}^{\infty}(z-d)^{3 / 2} \phi_{s}(z) d z
$$

The contact area is found to be the sum of individual contact patches $\pi \mathrm{a}_{\mathrm{i}}^{2}$ :

$$
A=\pi \sum_{i} a^{2}{ }_{i}=N \pi R \int_{d}^{\infty}(z-d) \phi_{s}(z) d z
$$

A general form of the contact integrals can be derived and generally denoted as GreenwoodWilliamson function:

$$
F_{n}=\int_{d}^{\infty}(z-d)^{n} \phi_{s}(z) d z
$$

Accordingly, the true contact stress $\sigma=\mathrm{F}_{\mathrm{N}} / \mathrm{A}$ can be expressed with the GreenwoodWilliamson functions and one obtains $\sigma \sim F_{1} / F_{3 / 2}$. If the friction force is assumed to be proportional to the real area of contact, it follows that the load dependence is given by the ratio $F_{1} / F_{3 / 2}$. However, this is valid for spherical asperities randomly distributed over a mean plane. For a real surface, the height distribution of contact patches determines the increase of friction force with increasing load. 


\section{(a) HERTZ}

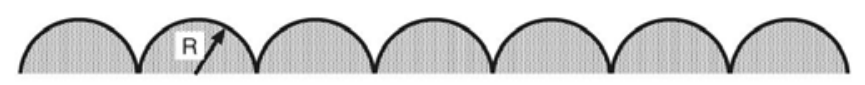

(b) GW

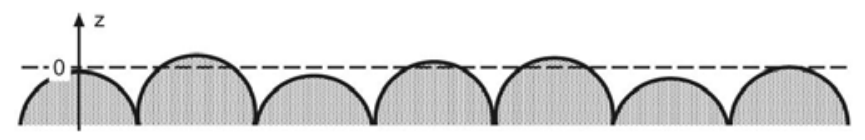

(c) Randomly rough

Figure 3.2: Schematic representation of the Hertz assumption (a), Greenwood-Williamson model (b) and a rough surface (c) ([49])

\subsubsection{Extension of the Greenwood-Williamson formulation}

A simple extension of the elastic contact derived by Greenwood and Williamson to rubber friction problems can be achieved by introducing the dynamic complex modulus $E^{*}(\omega)$ describing the frequency dependent behaviour of viscoelastic materials. In a first approximation, rubber can be assumed to be incompressible $\left(v_{1}=0.5\right)$. Also the Young modulus of rubber $E_{1}$ is smaller than the one from the rigid substrate by several orders of magnitude $\left(E_{1}<<E_{2}\right)$. Since one considers a rubber block sliding on rough surface, the radius of curvature of rubber is infinitely larger than the one from surface asperities, e.g. $R_{1} \gg R_{2}$. This leads to a simplification of the expression of contact parameters derived in the frame of the Hertz theory. Consequently, the normal force $F_{N}$ expressed in Equation (3.11) can be written as:

$$
F_{N}=\sum_{i=1}^{N} F_{n, i}=\frac{16}{9} N\left|E^{*}(\omega)\right| R^{1 / 2} \int_{d}^{\infty}(z-d)^{3 / 2} \phi_{s}(z) d z
$$

The decomposition of normal force has to be carefully treated for multiple-scale contact problems. Indeed, the theory of Greenwood and Williamson is based on the assumption that two neighbouring asperities act independently on the counter solid. For rough surfaces, this condition is clearly not satisfied at a certain length scale below which local deformation fields tend to overlap. Thus, statistically averaged and length scale dependent quantities like the 
mean asperity curvature or the real area of contact can not be evaluated within the frame of the Greenwood-Williamson theory in its basic form.

Nevertheless, a more detailed view of the sliding process shows that only the upper fraction of profile may actually deform the rubber during dynamic contact, so that macro-asperities appear in this case to act independently. Consequently, the applicability of the G-W approximation yields only for contact parameters governed by the largest roughness scales of the profile, for example the mean penetration depth $\left\langle z_{p}\right\rangle$ and the corresponding normal stress $\sigma_{0}$. For these quantities, the surface can reasonably be approximated by spheres corresponding to the largest asperity, e.g. with a diameter $\xi_{\perp}$ and separated by a distance $\xi_{\| l}$ (Figure 3.3).

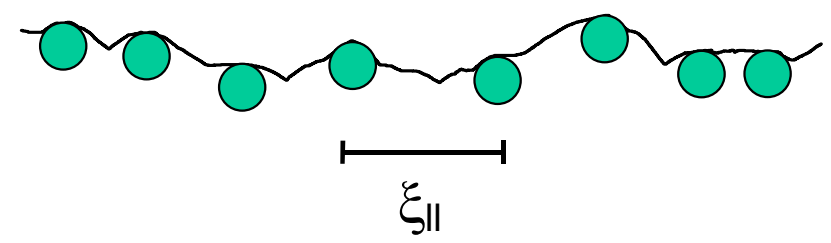

Figure 3.3: Approximation of a profile by spheres for the application of Hertz theory

The evaluation of contact forces requires the determination of the summit height distribution $\phi_{s}(z)$ characterising the fraction of profile contacting with the rubber. Based on an analytical formulation combined with a numerical procedure, a method has been proposed for the estimation of the summit height distribution $\phi_{s}(z)$. An affine transformation associated with an affine parameter $s$ is introduced to describe the shift of the original height distribution $\phi(z)$ towards higher region of the profile. Be the upper boundary $z_{\max }$ fixed, each point of the profile with a height $z$ will be transformed according to the following relationship:

$$
z_{s}=\frac{\left(z-z_{\max }\right)}{s}+z_{\max }
$$

where $z_{s}$ is the transformed height. The new standard deviation is given by:

$$
\tilde{\sigma}_{s}=\frac{\tilde{\sigma}}{s}
$$

and the new mean value $\left\langle z_{s}>\right.$ follows: 


$$
<z_{s}>=z_{\max }\left(1-\frac{1}{s}\right)
$$

On the other side, a numerical procedure calculates the local maxima distribution of a profile with various interval lengths. The principle is depicted in Figure 3.4. For small length intervals in the range of the measurement resolution, the maxima distribution tends to the original height distribution $\phi(z)$. By increasing the interval length, the procedure eliminates the valleys where no contact occurs and only retains the highest region of profile. In particular, the maxima distribution associated with an interval length $\xi_{\|}$corresponds to the approximation of Greenwood-Williamson where maxima are replaced by spheres.
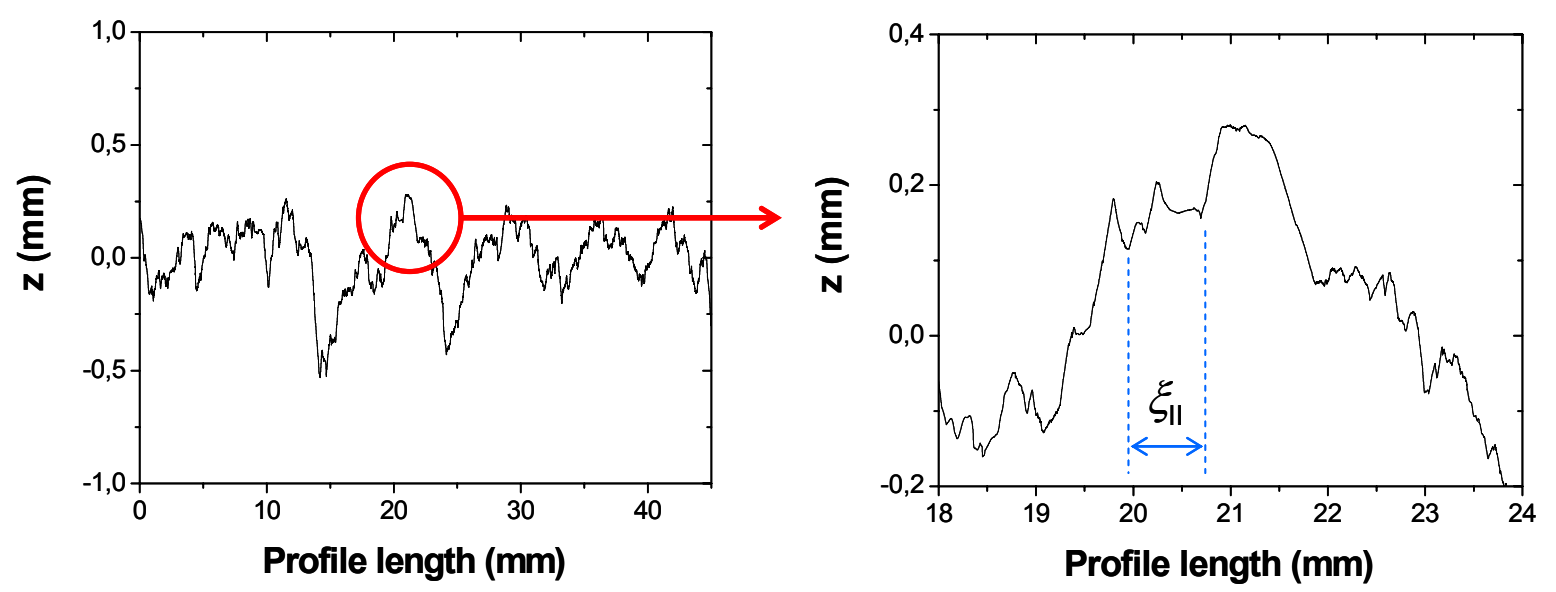

Figure 3.4: Original rough profile (left) and magnified region (right)

An example of the maxima distribution is shown in Figure 3.5 for a corundum surface with an interval length corresponding to the horizontal cut-off length $\xi_{\| l}$ of the surface. As expected, a shift of the mean value is observed which indicates that only the upper part of the profile is considered. Also, the shape of the distribution is narrower compared to the original distribution. As a comparison, the analytical affine transformed distribution is shown with an affine parameter found to be $s=1.20$. A fairly good agreement can be seen between both methods which allows an analytical formulation of the summit height distribution $\phi_{s}(z)=f(\phi(z))$, with the affine parameter as a characteristic of each surface. 


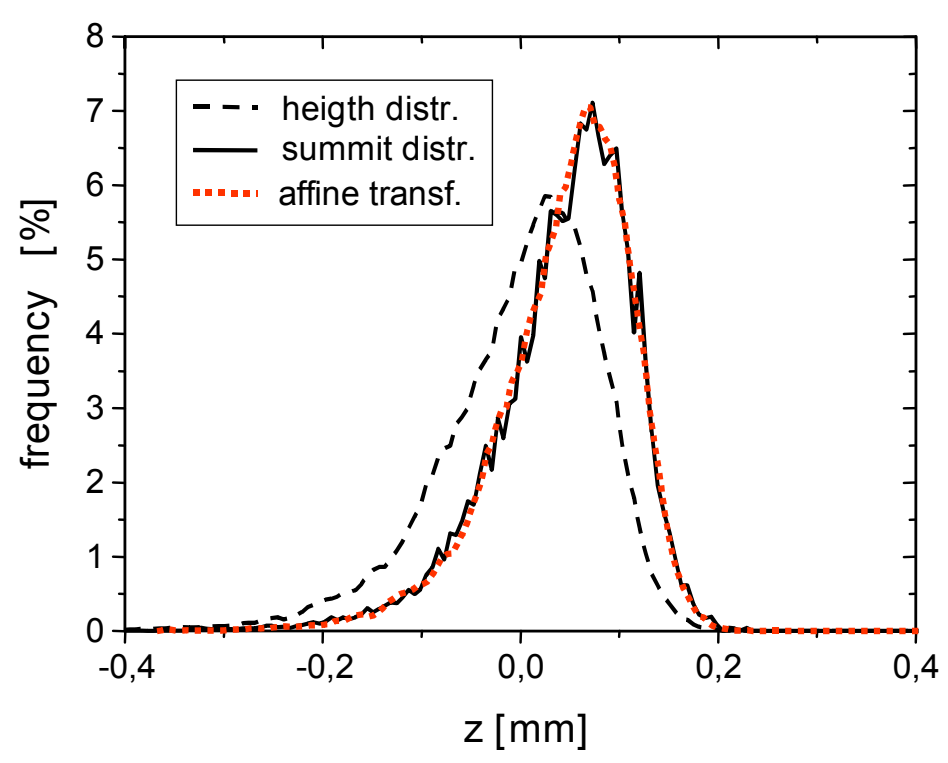

Figure 3.5: Comparison between the numerical maxima distribution and the analytical affine distribution for a corundum surface.

The numerical deviation between mean value and standard deviation for the maxima and affine distribution at different interval lengths is shown in Figure 3.6. The difference becomes higher with increasing interval length. However, in the range of the cut-off length $\xi_{\|}$where the profile is approximated by spheres, a maximum deviation of $5 \%$ is observed. This confirms the applicability of the proposed method for the determination of the summit height distribution and the estimation of contact parameters governed by the largest asperities of the surface.

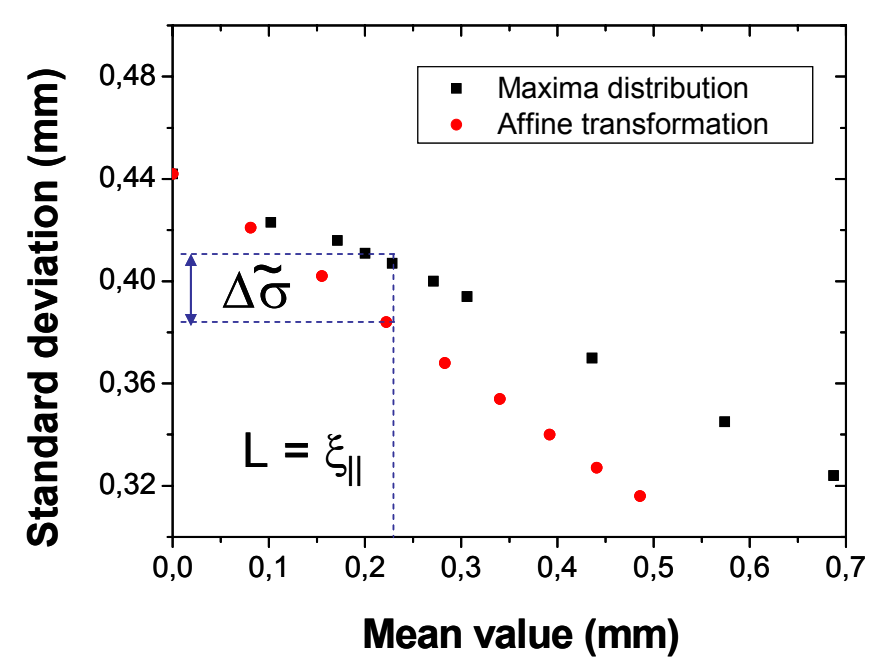

Figure 3.6: Deviation between the maxima and affine distribution 


\subsection{Self-affinity of rough surfaces}

Scaling properties of roughness are investigated in the next section in terms of self-affine behaviour. This feature is crucial for a multi-scale analysis of the contact between elastomers and rough surfaces and further the modelling of hysteresis and adhesion friction. In particular, a general formalism is derived for surfaces exhibiting at least one scaling regime which appears to be highly relevant for a fine characterisation of roughness and realistic predictions of sliding friction.

\subsubsection{Analytical formulation}

The fractal nature of many surfaces has been studied within the last twenty years [43][44][45][46]. It appears that most of engineered surfaces of practical importance exhibit a self-affine behaviour. The term self-affinity yields for objects which show an invariance of the morphology and statistical properties under anisotropic dilations [42]. For such surfaces, a vertical cross-section magnified by a scaling factor $\alpha$ in the $x y$ plane and by a factor $\alpha^{H}$ (where $0<\mathrm{H}<1$ ) in the perpendicular direction remains statistically invariant. $\mathrm{H}$ is denoted as the Hurst exponent and describes the degree of the surface irregularity. It is related to the local fractal dimension $\mathrm{D}$ by the equation $\mathrm{D}=3-\mathrm{H}$.

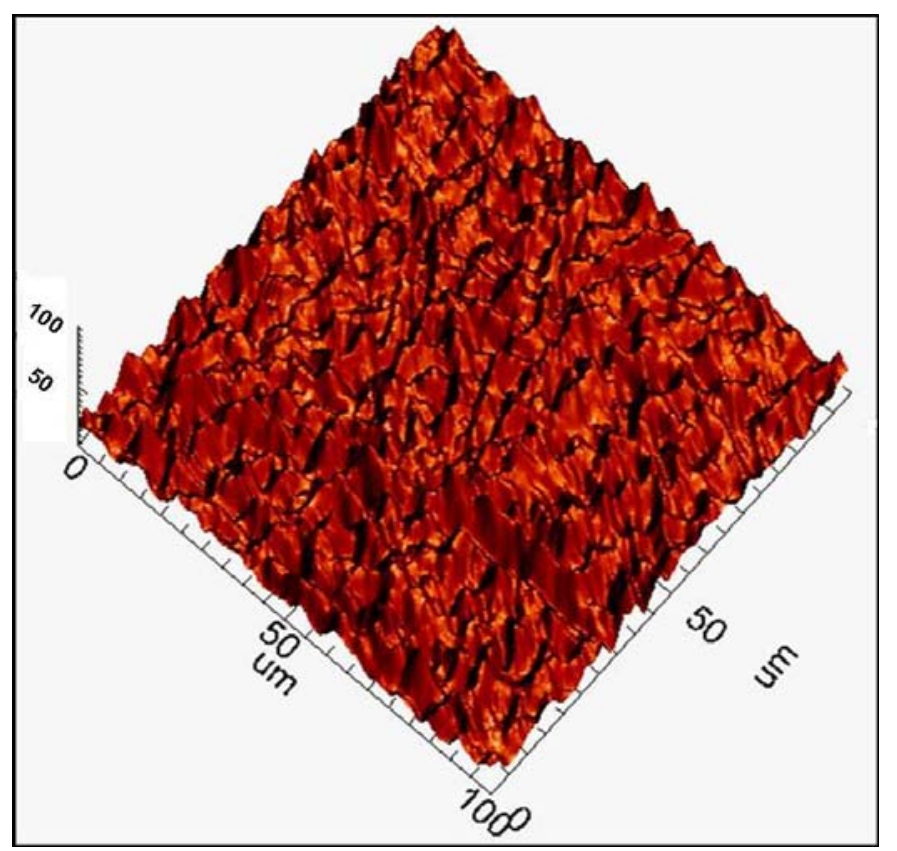

Figure 3.7: $\quad$ 3D-measurement of a smooth steel surface with Atomic Force Microscopy 
Many engineered surfaces show a self-affine behaviour within a defined wave length interval. The upper cut-off length is identified with the largest surface corrugations: for road surfaces, this corresponds to the limit of macrotexture, e.g. the aggregate size. On the other side, the lower cut-off length is fundamentally limited by the spatial resolution of the measuring equipment. The main experimental difficulty arises from the necessity to achieve a proper characterisation of the microtexture (micrometer range) while encompassing the largest asperities of the profile in the height up to a few millimetres. Consequently, the characterisation of self-affine properties is fundamentally limited by both the investigated surface and the measuring equipment. The length scale dependence of roughness is illustrated by a 3D reconstruction of a macroscopically smooth steel surface experimentally obtained by means of atomic force microscopy (Figure 3.7). A wavy structure of roughness can clearly be identified within the micrometer range and a self-affine behaviour is found for length scales down to $100 \mathrm{~nm}$.

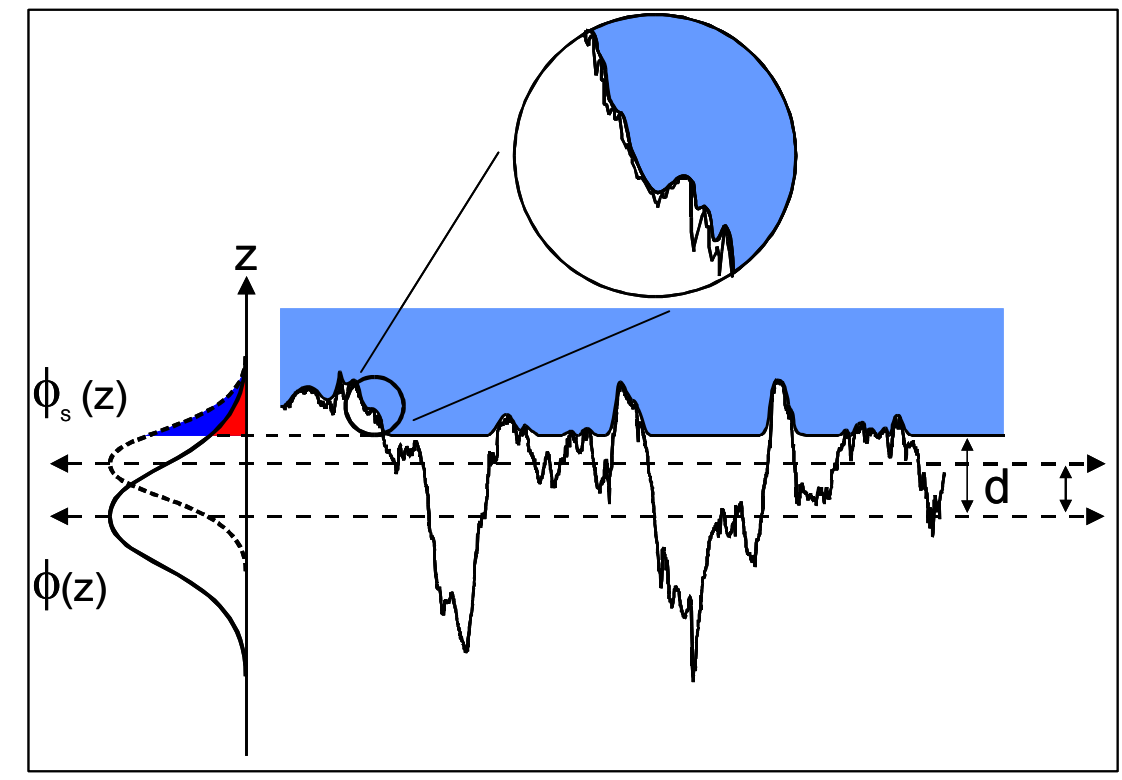

Figure 3.8: $\quad$ Rough profile with associated height distribution $\phi(z)$ and summit distribution $\phi_{\mathrm{s}}(\mathrm{z})$ (from [71])

The scaling properties of engineered surfaces imply a distribution of roughness over many length scales. Figure 3.8 clearly shows macroscopic asperities but a magnification of a profile region illustrates the existence of roughness at small length scales. Macroscopically rough surfaces like roads exhibit fractal dimensions comprised between $D=2$ and $D=2.5$. The value strongly depends on the type of roughness analysis performed [71]: needle measurements were found to provide a complete description of the roughness up to the microscale range but remain insufficient to fully characterise the largest asperities of road surfaces. On the contrary, laser measurements based on double triangulation allow measuring lengths up to $200 \mathrm{~mm}$ but are spatially limited by a horizontal resolution of $25 \mu \mathrm{m}$. 
The self-affine character of a surface is described by correlation functions, for instance the height-difference correlation function. It calculates the mean square height fluctuations of the surface with respect to the horizontal length scale $\lambda$ and is given by:

$$
C_{z}(\lambda)=<(z(x+\lambda)-z(x))^{2}>
$$

For self-affine surfaces, $\mathrm{C}_{\mathrm{z}}(\lambda)$ follows a power law with exponent $2 \mathrm{H}=6-2 \mathrm{D}$ on small length scales and reaches a plateau value $\xi_{\perp}$ corresponding to the vertical cut-off length of the profile. This value can be expressed as a function of the profile variance $\left(\sigma^{2}=\xi_{\perp}{ }^{2} / 2\right)$ and is thus directly related to the mean amplitude of roughness. The associated horizontal cut-off length is denoted as $\xi_{\|}$and physically interpreted as the size of the largest asperity of the profile. Therefore, it yields:

$$
C_{Z}(\lambda)=\xi_{\perp}^{2}\left(\frac{\lambda}{\xi_{\| I}}\right)^{6-2 D} \quad \text { for } \quad \lambda<\xi_{\|}
$$

The path of the height difference correlation function is schematically shown in Figure 3.9. From this function, a set of three descriptors completely characterises the surface. As previously mentioned, the self-affinity is fulfilled for real surfaces below both cut-off lengths generally over several orders of magnitude.

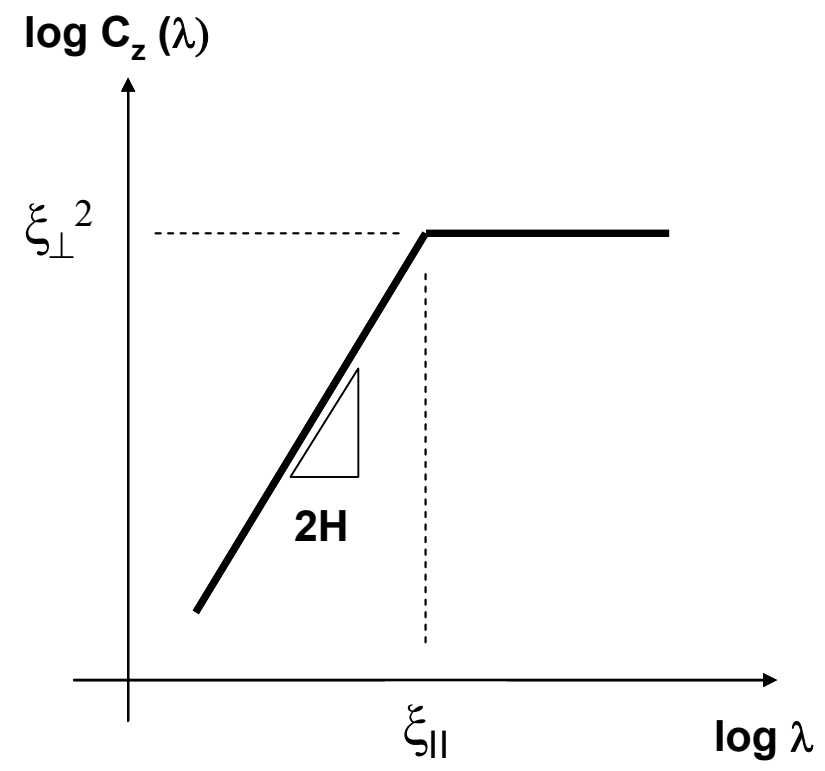

Figure 3.9: Height difference correlation function $C_{z}(\lambda)$ for a self-affine surface with corresponding set of descriptors 
A second approach consists in investigating statistical properties of a rough surface in the frequency space. For self-affine surfaces, the power spectrum density can be expressed as a function of the spatial frequency as following:

$$
S(f)=k\left(\frac{f}{f_{\min }}\right)^{-\beta}
$$

where $f_{\min }$ is the minimum frequency that corresponds to the inverse correlation length $\xi_{\|}$ and represents the maximum wave length of surface modulations.

The prefactor $k$ is termed topothesy and relates the surface descriptors by $k=\mathrm{H}_{\perp}{ }^{2} \xi_{\| 1}$. The exponent $\beta$ is given by: $\beta=2 \mathrm{H}+1=7-2 \mathrm{D}$. In other terms, both sets of descriptors are equivalent from a theoretical point of view. A numerical study of correlation functions based on a roughness analysis performed on real rough surfaces is presented in Chapter 5 .

\subsubsection{Extension to two scaling ranges}

Previous investigations showed that rough surfaces and in particular roads can be characterised by a fractal approach. The example of an asphalt surface shown in Figure 3.10 is based on a roughness analysis performed by means of laser measurements. A scaling behaviour is observed within the interval [25 $\mu \mathrm{m}-800 \mu \mathrm{m}$ ], giving access to a fractal dimension $D=2.3$ [63]. By extending the self-affine line up to the vertical cut-off length $\xi_{\perp}$, the horizontal cut-off length $\xi_{\|}$can be easily determined. However, a gradual discrepancy between the self-affine assumption and the statistical height difference correlation function can be clearly identified for length scales above $800 \mu \mathrm{m}$. This suggests that large length scales are likely to exhibit different morphological properties than the one characterising the range of microtexture. Recent investigations of tyre grip performance on a road surface (asphalt) confirm the deviation of statistical functions from a description based on a single scaling regime for large length scales [73]. 


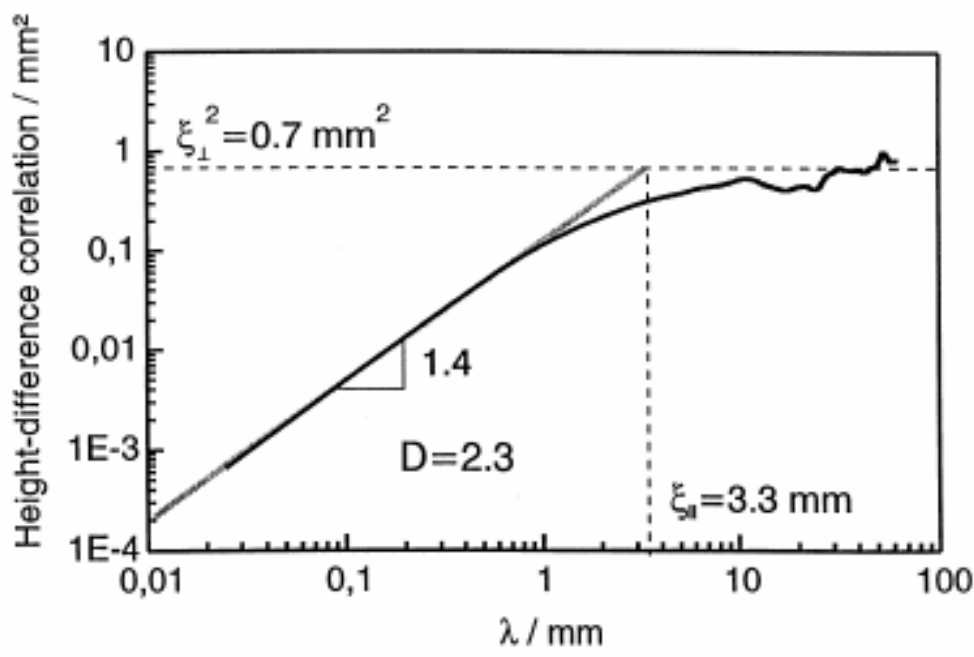

Figure 3.10: Height difference correlation function for an asphalt surface based on laser measurements and corresponding descriptors ([63])

This remark has practical implications on the modelling of roughness: indeed, road surfaces are known to be dominated by two main scaling regimes interpreted in terms of texture. The macrotexture is related to the roughness of the largest asperities - depending on granulate or aggregate size - while the microtexture rather describes their surface state (grain process, polishing, abrasion). Moreover, as presented in the former chapter, micro- and macrotexture are both associated with determining - but distinct - physical mechanisms regarding the dynamic contact tyre/road during friction processes under dry and wet conditions [5].

Consequently, a second scaling regime is introduced in order to obtain a precise description of scaling properties for large length scales. The path of the resulting height difference correlation function $C_{z}(\lambda)$ is schematically shown in Figure 3.11. It yields:

$$
C_{Z}(\lambda)=\xi_{\perp}^{2}\left(\frac{\lambda}{\xi_{\|}}\right)^{2 H_{1}} \text { for } \lambda_{2}<\lambda<\xi_{\|}
$$

Where $\lambda_{2}$ is the boundary length corresponding to the cross-over of both scaling ranges (Figure 3.11). For the second scaling range, the height difference correlation function $C_{z}(\lambda)$ is given by:

$$
C_{Z}(\lambda)=\xi_{\perp}^{2}\left(\frac{\lambda}{\lambda_{2}}\right)^{2 H_{2}}\left(\frac{\lambda_{2}}{\xi_{I I}}\right)^{2 H_{1}} \text { for } \lambda<\lambda_{2}
$$



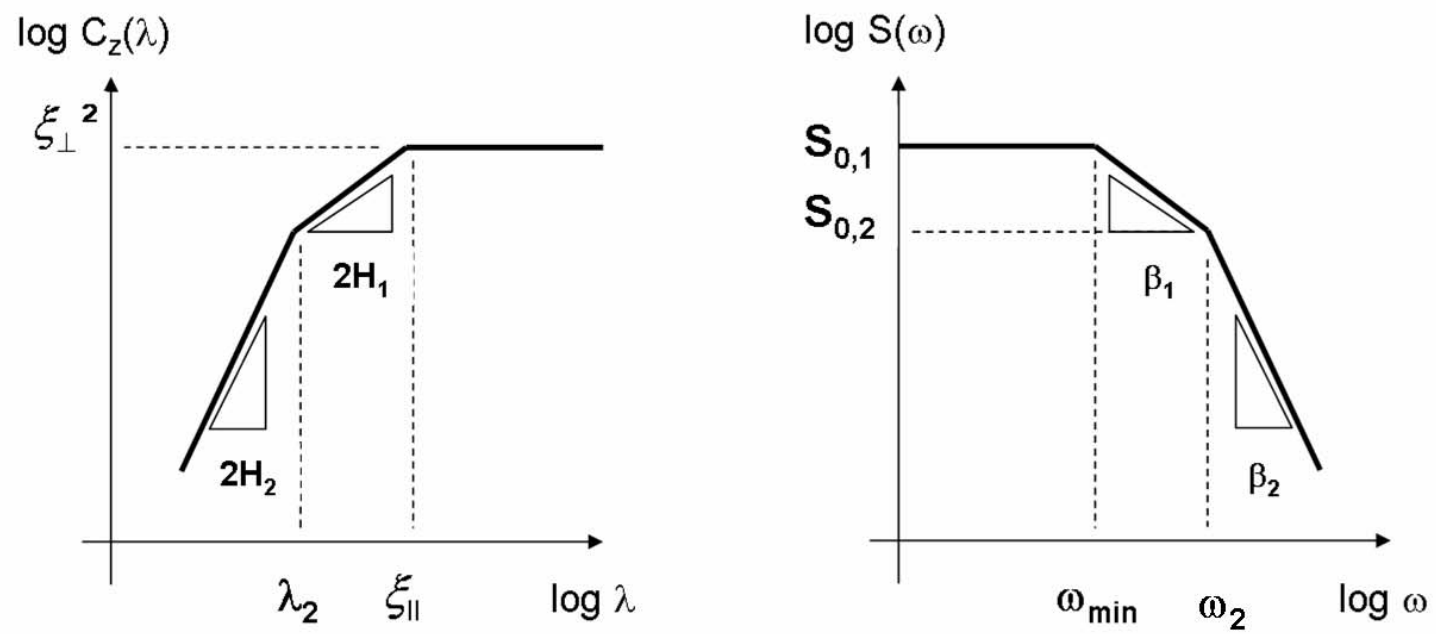

Figure 3.11: Schematic representation of the height difference correlation function $C_{z}(\lambda)$ and power spectrum density $S(\omega)$ with a two scaling ranges approach

A similar approach can be applied to the power spectrum density $S(\omega)$ in the frequency space (Figure 3.11). For the largest length scales, one obtains:

$$
\begin{aligned}
& S_{1}(\omega)=S_{o, 1}\left(\frac{\omega}{\omega_{\min }}\right)^{-\left(7-2 D_{1}\right)} \quad \text { for } \omega_{\min }<\omega<\omega_{2} \\
& S_{o, 1}=\frac{\left(3-D_{1}\right) \xi_{\perp}^{2}}{2 \pi v \xi_{\|}}
\end{aligned}
$$

where $\omega_{\min }=2 \pi \mathrm{v} / \xi_{\|}, \omega_{2}=2 \pi \mathrm{v} / \lambda_{2}$.

For the second scaling range, it yields:

$$
\begin{aligned}
& S_{2}(\omega)=S_{o, 2}\left(\frac{\omega}{\omega_{c}}\right)^{-\left(7-2 D_{2}\right)} \\
& S_{o, 2}=\frac{\left(3-D_{1}\right) \xi_{\perp}^{2}}{2 \pi v \xi_{\|}}\left(\frac{\omega_{\min }}{\omega_{c}}\right)^{\left(7-2 D_{1}\right)} \text { for } \omega_{2}<\omega
\end{aligned}
$$

where $S_{o, 2}$ is found to be directly related to the plateau value $S_{o, 1}$.

A general formulation of the height-difference correlation function and power spectrum density for $n$-scaling ranges is presented in Appendix $A$. 


\subsubsection{Real area of contact}

The fractal nature of rough surfaces has a strong impact on the level of contact area available during sliding friction. Indeed, since the roughness is distributed over many length scales, the amount of contact patches depends on the magnification at which the surface is observed (Figure 3.12). As a result, macroscopic observable contact patches can be decomposed into microscopic smaller ones and that the real area of contact only represents a small fraction of the apparent contact area. This is of considerable importance for rubber friction properties on rough surfaces which are basically determined by the ability to generate contact stresses within the contact region.

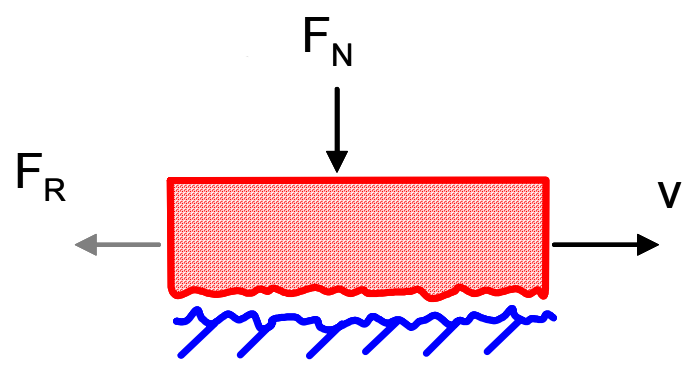

\section{Real area of contact $A_{c}$}

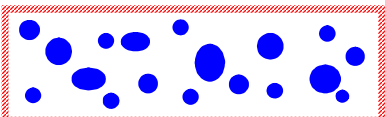

Figure 3.12: Schematic representation of contact patches distributed within the contact area during sliding process

The particular behaviour of the contact area as length scale dependent quantity can be highlighted by the yardstick method: it consists in covering the surface by gauges from various sizes and counting the number required for the formation of a packed monolayer. Figure 3.13 shows the principle of the yardstick method for two different gauge sizes. With decreasing radius, it can be seen that smaller cavities can be explored which leads to a higher observed contact area. This makes clear that the characterisation of a fractal object depends on the length scale at which it is measured.

The yardstick method can also be experimentally applied to smaller objects like carbon black aggregates: monolayer of different molecules (representing the gauges) can be isothermally absorbed on the filler surface and reveal the fractal nature of such nano-objects [47]. Such studies are from practical importance as the available area on a filler surface influences the wetting process with polymer chains during the mixing procedure and promotes the dispersion of filler particles within the polymer matrix. 


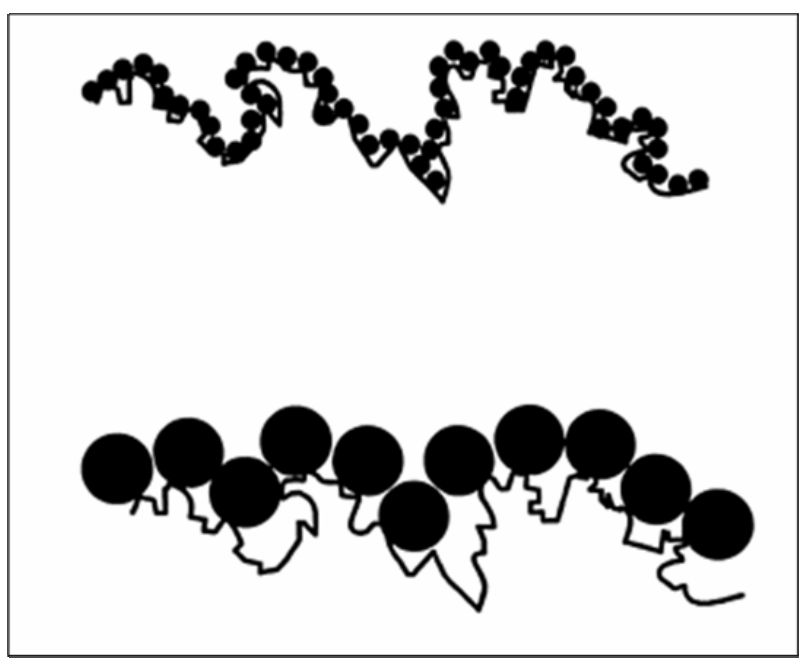

Figure 3.13: Measurement of profile length with different yardsticks (from [47])

For self-affine surfaces and below the cut-off length $\xi_{\| l}$, the number of gauges $N(\lambda)$ of size $\lambda$ obtained for a monolayer at a length scale $\lambda$ is directly related to the fractal dimension $\left(N \sim \lambda^{-D}\right)$. Thus, the real area of contact increases with decreasing gauge size as following:

$$
A_{c}(\lambda)=A_{0}\left(\lambda / \xi_{\|}\right)^{2-D} \quad \text { for } \lambda<\xi_{\|}
$$

For example, a brownian surface with $D=2.5$ observed at a length scale three decades below $\xi_{\text {II }}$ exhibits a contact area $A(\lambda) \sim 32 A_{0}$.

\subsection{Modelling of hysteresis friction}

Based on the formulated multi-scale contact analysis, generated hysteresis friction is analytically derived for elastomers sliding on rough surfaces. Contact parameters and the transition from a one to two-scaling-regimes approach are shown in details.

\subsubsection{One scaling range}

Hysteresis friction arises from the energy dissipation caused by the local deformation of rubber by surface asperities during sliding process. The dissipated energy can be generally written in the following form: 


$$
\Delta \tilde{E}_{\text {diss }}=\int_{0}^{v} \int_{0}^{T} \sigma \cdot \dot{\varepsilon} d t d^{3} x
$$

where $V$ is the excitated volume of the solid, $T$ is the sliding time, $\sigma$ and $\varepsilon$ the tensors of strain and gradient of deformation respectively. In the case of uniaxial deformation, the Fourier transform of $\sigma$ and $\varepsilon$ gives:

$$
\begin{gathered}
\sigma(t)=\int \hat{\sigma}(\omega) e^{i \omega t} d \omega \\
\varepsilon(t)=\int \hat{\varepsilon}^{*}(\omega) e^{-i \omega t} d \omega
\end{gathered}
$$

By introducing the Fourier representation of Dirac's $\delta$-function

$$
\delta\left(\omega-\omega^{\prime}\right)=\frac{1}{2 \pi} \int e^{i\left(\omega-\omega^{\prime}\right) t} d t
$$

and the complex modulus

$$
E^{*}(\omega)=E^{\prime}(\omega)+i E^{\prime \prime}(\omega)=\frac{\sigma^{*}(\omega)}{\varepsilon^{*}(\omega)}
$$

the dissipated energy can be written as

$$
\Delta E_{\text {diss }} \equiv<\Delta \tilde{E}_{\text {diss }}>=\frac{V}{2} T \int_{\omega_{\min }}^{\omega_{\max }} \omega E^{\prime \prime}(\omega) S(\omega) d \omega
$$

where $S(\omega)$ is the power spectrum density given by the Fourier transform of the autocorrelation of the roughness induced strain:

$$
<\hat{\varepsilon}(\omega) \hat{\varepsilon}^{*}\left(\omega^{\prime}\right)>=\frac{1}{2} S(\omega) \delta\left(\omega-\omega^{\prime}\right)
$$

By identifying the dissipated energy per time unit with the power generated by the hysteresis friction force $\left(\Delta \mathrm{E}_{\text {diss }} / \mathrm{T}=\mathrm{F}_{\mathrm{H}} \mathrm{v}\right)$, one obtains the following expression for the hysteresis friction coefficient [63]:

$$
\mu_{H} \equiv \frac{F_{H}}{F_{N}}=\frac{<\delta>}{2 \sigma_{o} V} \int_{\omega_{\min }}^{\omega_{\max }} \omega E^{\prime \prime}(\omega) S(\omega) d \omega
$$


where $\sigma_{0}$ is the nominal load, $v$ is the sliding velocity, $\langle\delta>$ is the excitated layer of rubber resulting from the dynamic contact with surface asperities, further assumed to be proportional to the mean penetration depth $\left\langle z_{p}\right\rangle$ of the rough substrate into the rubber. The lower boundary frequency is associated to the largest length scale of surface and is given by $\omega_{\min }=2 \pi \mathrm{v} / \xi_{\| 1}$. Note that the front factor in Equation (3.33) differs from the one derived in [60] due to a different normalization of the Fourier transform in Equations (3.27) and (3.28).

The estimation of the minimal length scale $\lambda_{\min }$ contributing to hysteresis friction (and the corresponding upper boundary frequency $\left.\omega_{\max }=2 \pi \mathrm{v} / \lambda_{\min }\right)$ is of considerable importance. Indeed, as presented in this section, the evaluation of the smallest cavity filled by rubber during dynamic contact leads to the determination of the contact area, e.g. the fraction of the rubber effectively involved in the frictional process. The situation is illustrated on Figure 3.14 for a rubber block pressed against a sinusoidal profile with a wave length $\lambda$.

The estimation of $\lambda_{\min }$ is based on an energy condition of elastic contact, stating that the sum of the elastic deformation work and adhesion energy upon contact should be larger that the elastically stored energy in the stress field of rubber [63]:

$$
\sigma(\lambda) \lambda^{2} h(\lambda)+\Delta \gamma \lambda h(\lambda) \geq E^{*}(\lambda) h^{3}(\lambda)
$$

Here, $\left|E^{*}(\lambda)\right|$ is the norm of the complex dynamic modulus at a frequency $\omega=2 \pi v / \lambda, v$ being the sliding velocity and $h(\lambda)$ the deformation of rubber while filling up a cavity of horizontal size $\lambda, \Delta \gamma$ is the change of surface energy per area unit of frictional pairing during sliding process.

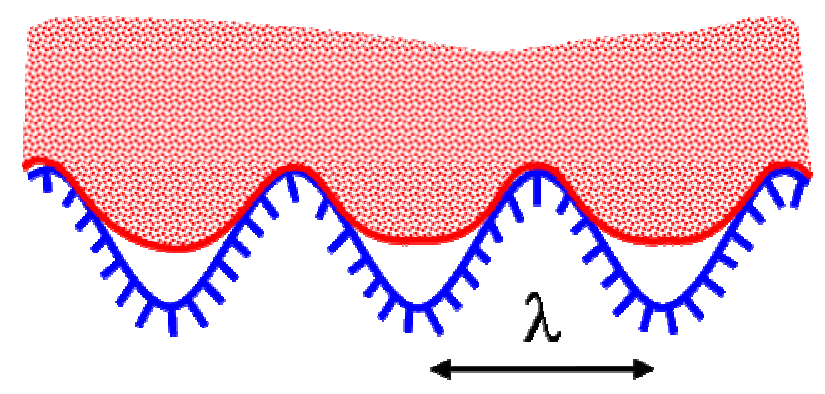

Figure 3.14: Deformation of the rubber on a sinusoidal profile

If the normal force is dominant, i.e. $\sigma(\lambda) \lambda \gg>\Delta \gamma$, the length scale interval $\left[\lambda_{\min }, \xi_{\| 1}\right]$ can be determined from Equation (3.34). In particular, the minimal length scale $\lambda_{\min }$ is given by [63]: 


$$
\frac{\lambda_{\min }}{\xi_{\|}}=\left(\frac{0.09 \pi s^{3 / 2} \xi_{\perp}(2 D-4)\left|E^{*}\left(\lambda_{\min }\right)\right| F_{o}(d / \tilde{\sigma})}{\xi_{\|}(2 D-2)\left|E^{*}\left(\xi_{\|}\right)\right| F_{3 / 2}\left(d / \tilde{\sigma}_{s}\right)}\right)^{\frac{1}{3 D-6}}
$$

where $F_{0}$ and $F_{3 / 2}$ denote the Greenwood-Williamson functions; also $E\left(\lambda_{\min }\right)=E\left(\omega_{\max }\right)$ and $s$ is the affine parameter characterising the summit height distribution at macroscopic length scales earlier presented in this chapter.

Using the scaling behaviour exhibited by the real area of contact in Equation (3.25), the calculation of the real area of contact $A_{c}$ leads to [63]:

$$
A_{c}\left(\lambda_{\min }\right)=A_{0}\left(\frac{1}{808 \pi} \frac{(2 D-4)^{2} \xi_{\|} F_{0}^{2}\left(\frac{d}{\tilde{\sigma}}\right) F_{3 / 2}\left(\frac{d}{\tilde{\sigma}_{s}}\right)\left|E^{*}\left(\frac{2 \pi v}{\xi_{\|}}\right)\right|}{s^{3 / 2}(2 D-2)^{2} \xi_{\perp}\left|E^{*}\left(\frac{2 \pi v}{\lambda_{\text {min }}}\right)\right|}\right)^{\frac{1}{3}}
$$

\subsubsection{Two scaling ranges}

It has been seen that the introduction of multi-fractality concepts implies a generalized formulation for both the height difference correlation function $C_{z}(\lambda)$ and the power spectrum density $S(\omega)$. Consequently, the hysteresis friction integral is decomposed into several terms corresponding to each scaling regime. Contact parameters like the mean penetration depth $\left\langle z_{p}\right\rangle$ calculated in the frame of the extended Greenwood-Williamson theory are not affected by the number a scaling regimes (no length scale dependence). In case of a two scaling ranges approach, the hysteresis friction coefficient $\mu_{H}$ is given by:

$$
\mu_{H} \equiv \frac{F_{H}}{F_{N}}=\frac{1}{2} \frac{<\delta>}{\sigma_{o} V}\left\{\int_{\omega_{\min }}^{\omega_{2}} d \omega \omega E^{\prime \prime}(\omega) \cdot S_{1}(\omega)+\int_{\omega_{2}}^{\omega_{\max }} d \omega \omega E^{\prime \prime}(\omega) \cdot S_{2}(\omega)\right\}
$$

where $\omega_{2}=2 \pi v / \lambda_{2}$ is determined via the cross-over of the two scaling regimes. $S_{1}(\omega)$ and $S_{2}(\omega)$ are the power spectrum density of macro- and microtexture, respectively, previously determined in Equations (3.23) and (3.24). A generalized formulation of the hysteresis friction coefficient and corresponding contact parameters for $n$-scaling ranges is presented in Appendix A. 
The amount of contact patches strongly depends on the magnification at which the contact is considered. This can be evidenced by the yardstick method which consists in counting the number of gauges $N(\lambda)$ of length scale $\lambda$ required to cover the surface. As a result, the contact area of self-affine surfaces increases with decreasing length scale according to a specific power law [63]. The introduction of a second scaling regime leads to an extension of the formulation for the contact area, and one obtains at a length scale $\lambda_{\min }$ :

$$
A_{c}\left(\lambda_{\min }\right)=A_{c, 0}\left(\frac{\lambda_{2}}{\xi_{I I}}\right)^{2-D_{1}}\left(\frac{\lambda_{\min }}{\lambda_{2}}\right)^{2-D_{2}}
$$

where $A_{c, 0}$ is the external contact area, $\xi_{\|}$accounts for the horizontal cut-off length, $\lambda_{2}$ is the cross-over length scale between macro- and microtexture, $D_{1}$ and $D_{2}$ correspond to the fractal dimension of macro- and microtexture, respectively.

In particular, the external contact area is defined as by the product of the weighed summit contact and the number of summits $n_{s} A_{0}[63]$ :

$$
A_{c, 0}=\frac{\pi}{2} \lambda_{c}^{2} n_{s} A_{0} F_{0}(t) \quad \text { with } \quad n_{s}=\frac{m_{4}}{6 \pi \sqrt{3} m_{2}}
$$

where $A_{0}$ is the nominal contact area, $F_{0}(t)$ is the Greenwood-Williamson function determined according to Equation (3.13), $\mathrm{n}_{\mathrm{s}}$ denotes the summit density and $\lambda_{\mathrm{c}}$ accounts for the lowest cut-off length of surfaces, typically $\lambda_{c} \sim 10^{-10} \mathrm{~m}$, where a cross-over from the fractal disorder to the atomic ordered structure appears. $m_{2}$ and $m_{4}$ are the second and fourth momenta of roughness spectra, respectively. For self-affine surfaces with a power spectrum density $S(f)$ characterised by two scaling ranges, a general form of the momenta is given by:

$$
m_{n}=\int_{f_{\min }}^{f_{2}} f^{n} S_{1}(f) d f+\int_{f_{2}}^{f_{\max }} f^{n} S_{2}(f) d f
$$

where $f_{\min }=1 / \xi_{\|}, f_{2}=1 / \lambda_{2}$ and $f_{\max }=1 / \lambda_{\min } . S_{1}(f)$ and $S_{2}(f)$ corresponds to the power spectrum density of macro- and microtexture, respectively.

According to Equation (3.23) and (3.24), the calculation of $m_{2}$ and $m_{4}$ leads to the following expression for the summit density $n_{s}$ : 


$$
n_{s}=\frac{1}{6 \pi \sqrt{3} \lambda_{c}^{2}}=\frac{\frac{1}{5-\beta_{1}}\left(\frac{\lambda_{c}}{\lambda_{2}}\right)^{5}+\frac{1}{5-\beta_{2}}\left(\frac{\lambda_{c}}{\lambda_{2}}\right)^{\beta_{2}}}{\frac{1}{3-\beta_{1}}\left(\frac{\lambda_{c}}{\lambda_{2}}\right)^{3}+\frac{1}{3-\beta_{2}}\left(\frac{\lambda_{c}}{\lambda_{2}}\right)^{\beta_{2}}}
$$

where $\beta_{1}=7-2 D_{1}$ and $\beta_{2}=7-2 D_{2}$, with $D_{1}$ and $D_{2}$ accounting for the fractal dimension of macro- and microtexture, respectively.

Accordingly, the real area of contact $A_{c}\left(\lambda_{\min }\right)$ associated with the contact interval $\left[\lambda_{\min }, \xi_{\| 1}\right]$ can be rewritten as:

$$
A_{c}\left(\lambda_{\min }\right)=\frac{A_{0} F_{0}(t)}{12 \sqrt{3}} \frac{\frac{1}{5-\beta_{1}}\left(\frac{\lambda_{c}}{\lambda_{2}}\right)^{5}+\frac{1}{5-\beta_{2}}\left(\frac{\lambda_{c}}{\lambda_{2}}\right)^{\beta_{2}}}{\frac{1}{3-\beta_{1}}\left(\frac{\lambda_{c}}{\lambda_{2}}\right)^{3}+\frac{1}{3-\beta_{2}}\left(\frac{\lambda_{c}}{\lambda_{2}}\right)^{\beta_{2}}}\left(\frac{\lambda_{2}}{\xi_{I I}}\right)^{2-D_{1}}\left(\frac{\lambda_{\text {min }}}{\lambda_{2}}\right)^{2-D_{2}}
$$

By assuming that the elastic deformation work due to the normal force is predominant over the adhesion energy, the minimal length scale of the contact interval $\left[\lambda_{\min }, \xi_{\| 1}\right]$ is given by [63]:

$$
\lambda_{\min } \cong\left(\frac{\left|E\left(\lambda_{\min }\right)\right| C_{z}\left(\lambda_{\min }\right)}{\sigma\left(\lambda_{\min }\right)}\right)^{1 / 2}
$$

where $E\left(\lambda_{\min }\right)$ is the dynamic modulus at a frequency $\omega=2 \pi v / \lambda_{\min }$, $v$ being the sliding velocity. $C_{z}\left(\lambda_{\min }\right)$ accounts for the height difference correlation function at a length scale $\lambda_{\text {min }}$, $\sigma\left(\lambda_{\min }\right)$ is the true mean contact stress defined by:

$$
\sigma\left(\lambda_{\min }\right)=\frac{F_{N}}{A_{c}\left(\lambda_{\min }\right)}=\frac{\sigma_{0} A_{0}}{A_{c}\left(\lambda_{\min }\right)}
$$

By combining Equations (3.42), (3.43) and (3.44), the smallest length scale $\lambda_{\text {min }}$ contributing to hysteresis friction with a two-scaling-ranges approach is found as

$$
\frac{\lambda_{\min }}{\xi_{\|}} \cong\left(\left(\frac{\lambda_{2}}{\xi_{\|}}\right)^{3\left(D_{2}-D_{1}\right)} \frac{0.09 \pi s^{3 / 2} \xi_{\perp}\left|E\left(\lambda_{\min }\right)\right| F_{o}(t) \tilde{n}_{s}}{\xi_{\|}\left|E\left(\xi_{\|}\right)\right| F_{3 / 2}\left(t_{s}\right)}\right)^{\frac{1}{3 D_{2}-6}}
$$




$$
\text { with } \tilde{n}_{s}=6 \pi \sqrt{3} \lambda_{c}^{2} n_{s}
$$

If Equation (3.45) is inserted into Equation (3.42), the corresponding real area of contact $A_{c}\left(\lambda_{\min }\right)$ is given by:

$$
A_{c}\left(\lambda_{\min }\right) \approx A_{0}\left(\frac{\xi_{\| 1} F_{o}^{2}(t) F_{3 / 2}\left(t_{s}\right)\left|E\left(\xi_{\| 1}\right)\right| \tilde{n}_{s}{ }^{2}}{808 \pi s^{3 / 2} \xi_{\perp}\left|E\left(\lambda_{\min }\right)\right|}\right)^{\frac{1}{3}}
$$

where a short analysis shows that $\tilde{n}_{s} \sim\left(3-\beta_{2}\right) /\left(5-\beta_{2}\right)$, e.g. the correction term is solely influenced by the fractal dimension of microtexture.

\subsection{Modelling of adhesion friction}

Beside hysteresis friction, a modelling of adhesion friction is presented in the next section. It relies on the calculated real area of contact combined with a velocity dependent interfacial shear strength which reflects the kinetics of peeling effects of rubber on the smallest length scales during the sliding process.

\subsubsection{Role of contact area}

As presented in Chapter 2, adhesion can even take place during rubber sliding friction on wet rough surfaces. The presence of lubricant is a necessary but not sufficient condition for the suppression of adhesion [17]. The breakage of lubricant film during the sliding process is activated by the surface microtexture and might lead to the formation of statistically distributed islands of dry contact under wet conditions [5]. This mechanism appears to be strongly dependent on the nature of the lubricant, morphology of the roughness and can be hindered by detergents which are known to prevent intimate contact between rubber and rough substrate. Under dry conditions, both hysteresis and adhesion components contribute to the frictional process.

Generally, adhesion plays a role during friction if an intimate contact between two solids takes place at the interface. The amount, structure and nature of contact patches control the level of adhesion. A simple formulation of the adhesion friction is given by: 


$$
\mu_{\text {Adh }}=\frac{F_{\text {Adh }}}{F_{N}}=\frac{\tau_{s}}{\sigma_{o}} \cdot \frac{A_{c}}{A_{o}}
$$

where $\tau_{s}$ is the true interfacial shear strength, $\sigma_{0}$ is the load, $A_{0}$ and $A_{c}$ correspond to the nominal and real contact area, respectively.

Hence, the adhesion appears to be directly proportional to the real area of contact $A_{c}$. The true interfacial shear strength $\tau_{s}$ describes the local force required to break contact junctions.

\subsubsection{Kinetics of the interfacial shear strength}

The formation and breakage of contact patches between rubber-like materials and hard substrates has been extensively studied for rolling friction, rebound and peel-off experiments. When the two solids are put into contact, the observed contact radius is higher than the Hertz which indicates that attractive forces occur and can be considered via the surface energy $\Delta \gamma$ of the interface [35]. Also, when the two solids are put apart, the peeling-off process exhibits a strong dependence on the rubber type, temperature and rate of separation. This is due to the existence of high strain regions located at the periphery of the contact zone which are driven by the rubber viscoelastic properties during the breakage of contact junctions [25].

Similarities can be found with rubber sliding friction on rough surfaces. Indeed, both processes of adhering and peeling-off occur during dynamic contact. Let us consider an element of a rubber solid sliding over a single asperity: when the element comes into the entrance zone, an adhering process occurs due to the attractive forces with the spherical asperity located at the trailing edge of the contact. This is followed by the deformation of the bulk material associated with the hysteresis friction. Finally, at the leading edge, a breakage of the contact is observed via a peeling-off process resulting in local energy dissipation. This mechanism is schematically illustrated in Figure 3.15.

By superposing a second wave length on the asperity, it can be seen that the peeling-off process occur over the whole contact zone and not only at the leading edge. Since real surfaces are rough over many length scales, the mechanisms described above are statistically distributed in the contact area. The formation and breakage of contact patches is expected to be controlled by the viscoelastic properties of the rubber as it corresponds to a mechanical stretching and relaxation process of polymer chains. 


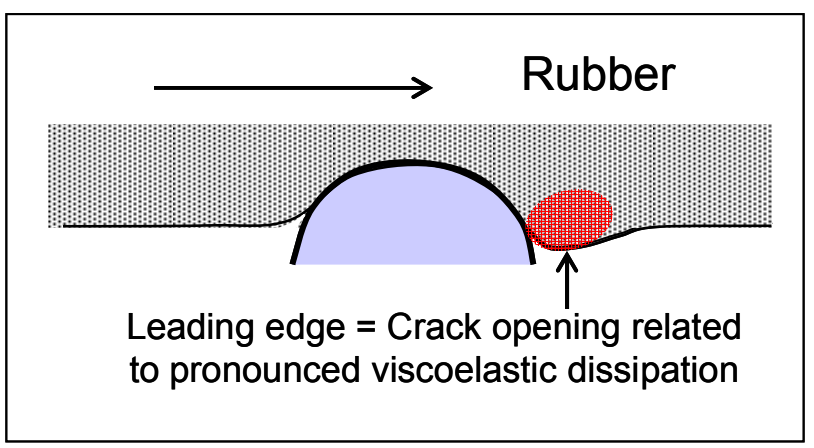

Figure 3.15: Schematic view of local peeling effects occurring during dry sliding friction

The rate dependence of peeling-off experiments between rubber-like materials and a blunt asperity has been taken in account via the introduction of an effective surface energy term $\Delta \gamma_{\text {eff }}$ describing the viscoelastic nature of such processes [21]. At low rates of separation, the effective surface energy reaches a constant value corresponding to the static surface energy $\Delta \gamma_{0}$. By increasing the rate of separation, $\Delta \gamma_{\text {eff }}$ goes through a modulus-like transition and follows a power law until a critical velocity $v_{c}$ assumed to depend on the nature of the polymer and the filler content (Figure 3.16). The step height of the transition is given by the one observed during mechanical spectroscopy, e.g. $E_{\propto} / E_{0}$ (dynamic modulus in glassy and rubbery state)

The surface energy $\Delta \gamma$ is related to the true interfacial shear strength $\tau_{s}$ by: $\tau_{s}=\Delta \gamma \cdot l_{s}^{-1}$, where $I_{s}$ is a typical length scale at which such processes occur.

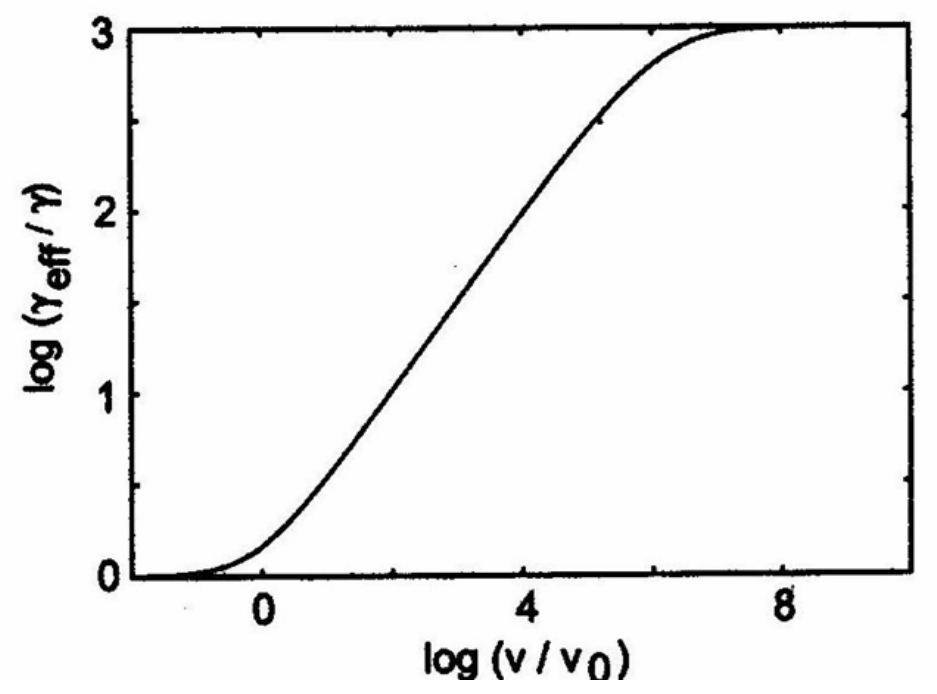

Figure 3.16: Effective surface energy $\Delta \gamma_{\text {eff }}$ as a function of the sliding velocity $v$ (from [50])

From these considerations, an empirical formulation for the velocity dependence of the true interfacial shear strength $\tau_{s}$ can be derived and is given by: 


$$
\tau_{s}=\tau_{s, 0}\left(1+\frac{E_{\infty} / E_{0}}{\left.\left(1+\left(v_{c} / v\right)\right)^{n}\right)}\right)
$$

where $n$ is a material dependent exponent, $\tau_{s, 0}$ is the interfacial shear strength in the limit of very low velocities, $v_{c}$ is the critical velocity above which the true interfacial shear strength $\tau_{s}$ is constant, $E_{\infty} / E_{0}$ is the step height of the dynamic modulus between rubbery and glassy state.

A numerical application of the true interfacial shear strength $\tau_{s}$ is schematically presented in Figure 3.17.

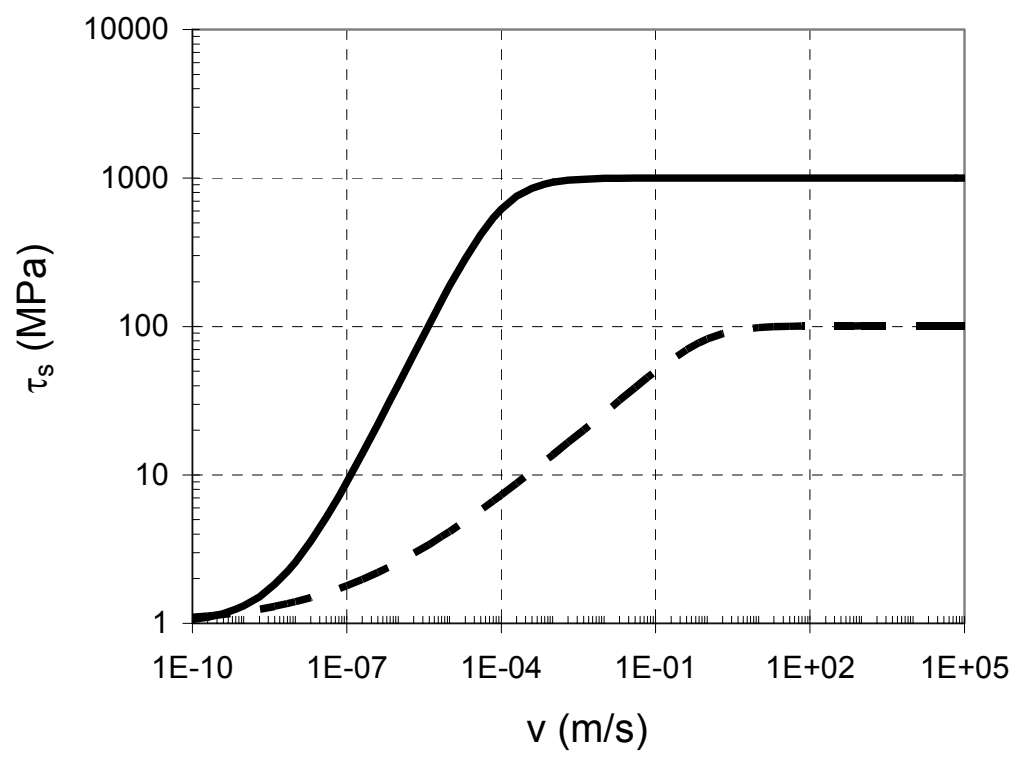

Figure 3.17: Simulated interfacial shear strength $\tau_{\mathrm{s}}$ according to Equation (3.48) as a function of the sliding velocity with $\tau_{s, 0}=1$. Full curve: $n=0.7, v_{c}=10^{-4} \mathrm{~m} / \mathrm{s}, E_{\infty} / E_{0}=1000$. Dashed curve: $n=0.3, v_{c}=1 \mathrm{~m} / \mathrm{s}, E_{\infty} / E_{0}=100$ 


\section{Materials and experimental methods}

The applicability of friction modelling has been investigated on model elastomer systems based on commercial polymers and modern fillers. Experimental methods include relaxation spectroscopy for the evaluation of mechanical properties in the high frequency region, roughness analysis required for the length-scale dependent characterisation of surfaces, photogrammetry related to contact analysis and two test rigs dedicated to friction measurements.

\subsection{Materials}

A systematic variation of the formulation was carried out to separate the effect of the polymer matrix and the filler system and their impact on friction properties. Samples include a solution-styrene-butadiene rubber S-SBR with $50 \mathrm{wt} . \%$ vinyl and $25 \mathrm{wt} \%$ styrene (BUNA VSL 5025-0 HM) often used for tread compounds, a second solution-styrene-butadiene rubber S-SBR with 25 wt.\% vinyl content and also an emulsion-styrene-butadiene rubber ESBR 1500. The polymers were mixed with two different filler systems, namely carbon black N339 and silica GR7000 with a coupling agent Coupsil 8113. The Coupsil 8113 consists of silica particles coated with a bifunctional silane, ensuring a reasonable level of dispersability during mixing and formation of covalent bonds with the polymer during vulcanisation. The formulation of compounds is completed with anti-aging (1.5 phr IPPD) as well as processing agents ( 1 phr Stearic Acid and 3 phr Zinc Oxide). In order to achieve a sufficient level of elasticity, both polymers were cross-linked in a steam press with 1.7 phr sulphur in combination with $2.5 \mathrm{phr}$ accelerator (CBS). The full formulations of the materials are listed in Table 4.1.

The unfilled polymers were mixed on a roller mill for 5 minutes while the filled systems had a two step mixing stage with first 5 min in an internal mixer followed by 5 minutes on the roller mill. The curing time was measured on a Monsanto rheometer at a temperature of $160^{\circ} \mathrm{C}$ and corresponds to $t_{95}$, i. e. the required time to reach $95 \%$ of the maximum torque deduced from the vulcameter curves of each compound. Test samples were finally vulcanised in a heating press under 200 bars pressure at a curing temperature of $160^{\circ} \mathrm{C}$. 


\begin{tabular}{|c|c|c|c|c|c|c|c|}
\hline \multirow{2}{*}{$\begin{array}{c}\text { Polymer } \\
\text { Content (phr) }\end{array}$} & \multicolumn{3}{|c|}{ S-SBR 5025} & \multicolumn{3}{|c|}{ S-SBR 2525} & \multirow{2}{*}{$\begin{array}{c}\text { E-SBR } 1500 \\
100\end{array}$} \\
\hline & 100 & 100 & 100 & 100 & 100 & 100 & \\
\hline Filler & None & N339 & GR7000 & None & N339 & GR7000 & N339 \\
\hline Content (phr) & 0 & 60 & 60 & 0 & 60 & 60 & 60 \\
\hline Silane & - & - & 5 & - & - & 5 & - \\
\hline Zinc Oxyd & \multicolumn{7}{|c|}{3} \\
\hline Stearic Acid & \multicolumn{7}{|c|}{1} \\
\hline IPPD & \multicolumn{7}{|c|}{1.5} \\
\hline CBS & \multicolumn{7}{|c|}{2.5} \\
\hline$S$ & \multicolumn{7}{|c|}{1.7} \\
\hline
\end{tabular}

Table 4.1: $\quad$ Formulation of rubber compounds

\subsection{Roughness analysis}

The characterisation of rough surfaces is a determining step for the calculation of fractal descriptors and the resulting hysteresis friction. Due to spatial limitations, high-resolution experimental methods like Atomic Force Microscopy (AFM) can not be applied for rough surfaces. A complete measuring of both micro- and macrotextures requires a sufficient vertical range of stylus movements combined with a high horizontal resolution.

The Talysurf 100 is a profilometer measuring system which detects and evaluates curvature, inclination, roughness and waviness of surfaces with a single traverse across the component. The Talysurf 100 is composed of a probe head equipped with a sapphire ball stylus. The related arm includes an inductive gauge with a pivoted and balanced beam to allow measurement in any attitude. It allows a vertical range of movements of $+/-1 \mathrm{~mm}$, which is optimal for surfaces like rough granite or fine asphalt. The horizontal interval of sampling data is $\Delta x=0.5 \mu \mathrm{m}$, the vertical resolution is $\Delta z=16 \mathrm{~nm}$. The measured data can be exported and displayed on a processor control module. The experimental set-up is schematically presented in Figure 4.1.

Surface measurements were carried out until the maximal length of the traverse, e.g. $50 \mathrm{~mm}$. In order to ensure a good statistic of the roughness analysis, the measurement was repeated 15 times for each surface in different directions. 


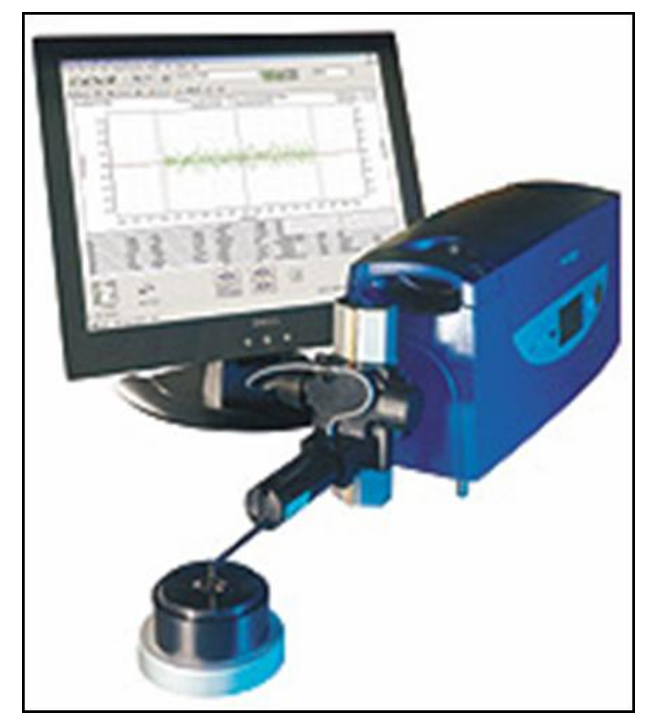

Figure 4.1: Experimental set-up of a profilometry measurement

\subsection{Relaxation spectroscopy}

Relaxation spectroscopy has been a useful technique over the last decades to investigate the relationship between the microstructure and some macroscopic properties of polymeric materials. The dynamic mechanical analysis and the dielectric spectroscopy are probably the two most used techniques to provide some information on the frequency and temperature dependent relaxation processes occurring in the rubber matrix. In both cases, specific fluctuations of the material response are detected using small perturbations of the external force field.

\subsubsection{Dynamic Mechanical Thermal Analysis (DMTA)}

The mechanical response of rubber in the high frequency range is strongly related with the level of hysteresis friction. From the experimental point of view, classical testing equipments can reasonably generate mechanical excitation up to $1 \mathrm{kHz}$. For an estimation of the high frequency dynamic behaviour, the correspondence between time, e.g. frequency, and temperature is used. This allows the construction of master curves, e.g. the dynamic mechanical modulus $\mathrm{G}^{*}$ as a function of the frequency.

The dynamic mechanical analysis was firstly performed on a dynamic analysator ARES from the company Rheometrics (Figure 4.2). The principle is based on a clamped strip specimen sinusoidally stretched under torsion at a constant deformation. The resulting torque is 
measured up to $2.10^{-3} \mathrm{Nm}$ as well as the shift angle between imposed strain and corresponding stress. This allows the calculation of both storage and loss moduli, G' and G" respectively. As long as the deformation is small compared to the specimen height, torsion can be assimilated to shear deformation. The measuring frequency range is comprised between $f=0.001 \ldots 80 \mathrm{~Hz}$ while the deformation range goes from $\varepsilon=0.5 \%$ up to $\varepsilon=3.5 \%$. A static pre-tension of $150 \mathrm{~g}$ is applied on the specimen to ensure sufficient conditioning over the whole range of temperature. The experimental set-up finally involves a temperature chamber which enables dynamic measurements at temperatures comprised between $\mathrm{T}=-100^{\circ} \mathrm{C}$ and $\mathrm{T}=100^{\circ} \mathrm{C}$.

For construction of master curves, frequency and temperature dependent sweeps were applied between $f=0.015$ and $f=15 \mathrm{~Hz}$ at various strain amplitudes.
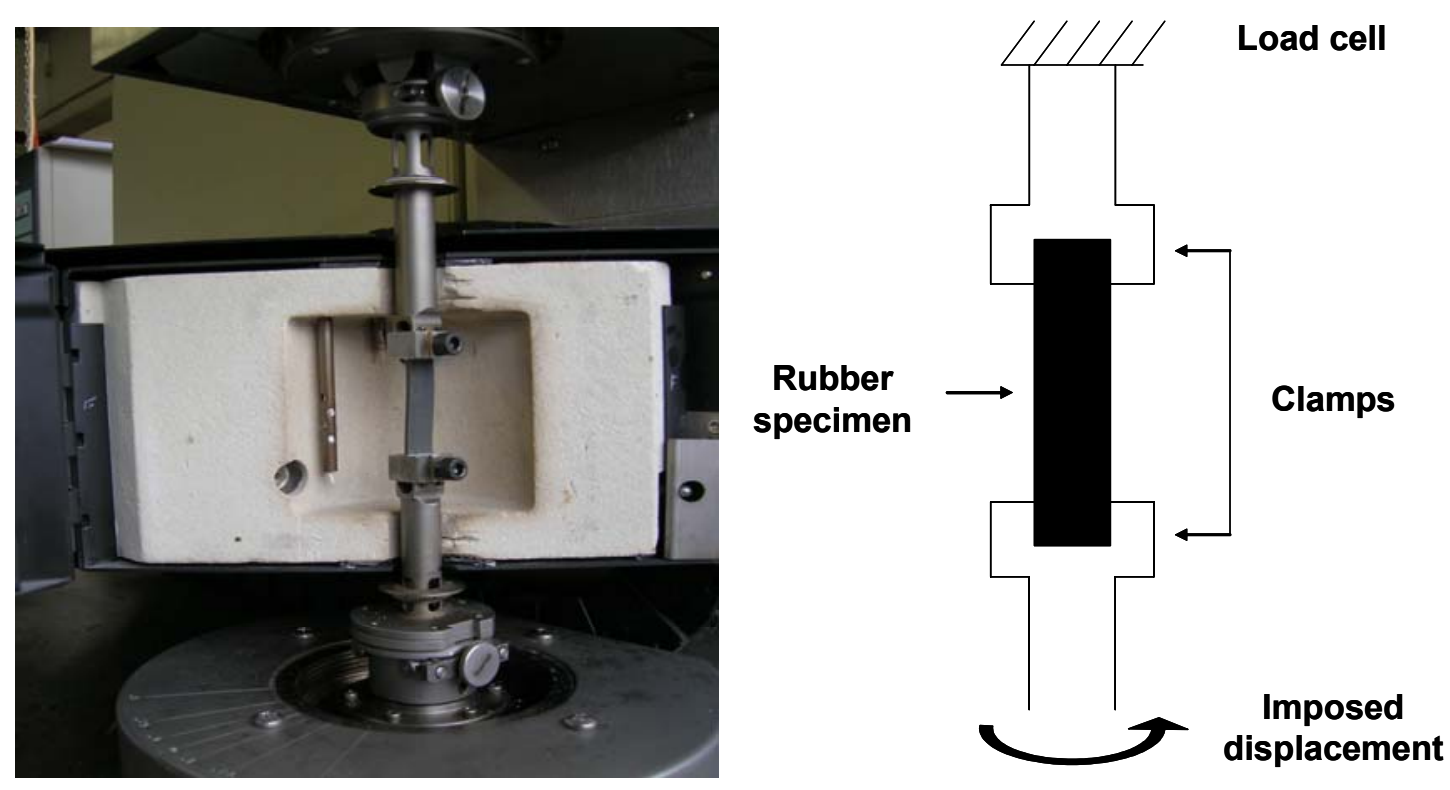

Figure 4.2: Experimental set-up of the Ares mechanical spectrometer

An extension of the experimental conditions was enabled through dynamic measurements carried out on uniaxial MTS servo-hydraulic testing equipment MTS 831.50 (Figure 4.3). It allows a temperature and frequency dependent characterisation on various materials up to 1 $\mathrm{kHz}$ under load or displacement control. The main advantage of this method is the possibility to test rubber specimen under realistic conditions: for friction applications, dumbbell geometry can be pre-compressed at a constant displacement and then harmonically deformed around this position. Dumbbell specimen allows compression up to $30 \%$ without any significant barrelling compared to other cylindrical test geometry. The evolution of dynamic properties could be followed with a special elastomer module (TestWare SX) of the TestStar control software. 
As before, the construction of master curves is based on frequency sweeps between $f=0.02 \mathrm{~Hz}$ and $f=10 \mathrm{~Hz}$ applied at various temperatures. The level of pre-compression was fixed at $10 \%$ and $20 \%$, the strain amplitude was gradually increased (peak to peak) at $\varepsilon=2$, 6 and $10 \%$.
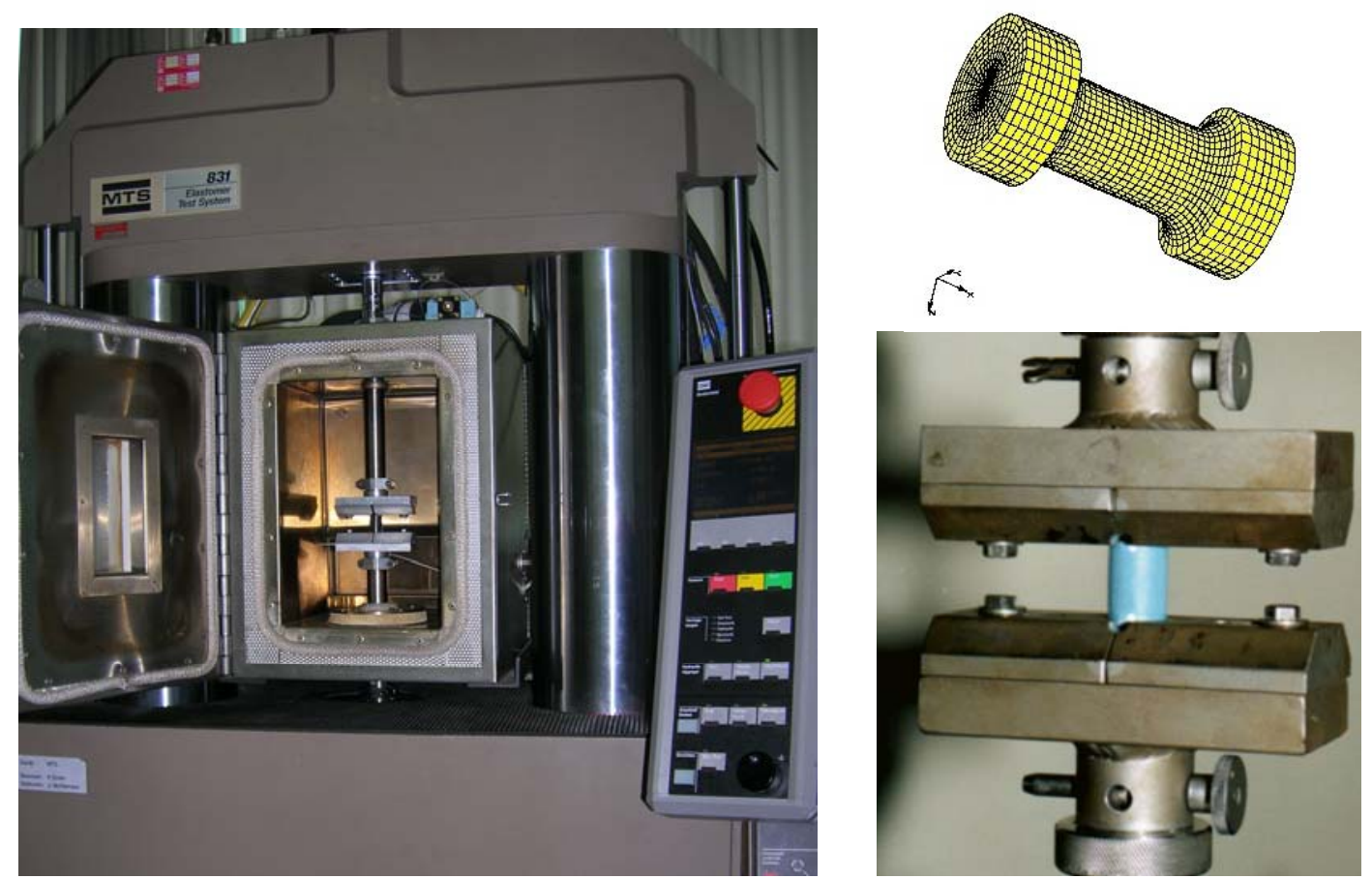

Figure 4.3: Experimental set-up of MTS servo-hydraulic testing equipment for dynamic mechanical analysis on dumbbell specimen

\subsubsection{Dielectric spectroscopy}

A second measurement technique for investigating the relaxation processes of rubber materials is achieved by dielectric spectroscopy. In this case, the interaction between the material and a dynamic electric field is measured over a broad range of frequency and temperature. Two interaction mechanisms can be observed, namely polarization associated with the orientation of permanent dipoles (reversible storage of field energy), and conduction related with dissipative effects. As for mechanical spectroscopy, molecular motions of molecules or segments within the material are detected under external force field. The nature of the interactions results in a higher sensitivity of dielectric measurements: thus a resolution of $10^{-5}$ can be achieved on the dielectric loss factor (DMA: $10^{-3}$ ).

The measurements of dielectric spectroscopy were performed with a broadband dielectric spectrometer BDS 40 manufactured by the company Novocontrol $\mathrm{GmbH}$. It provides a bandwidth of eleven decades, from $10^{-4} \mathrm{~Hz}$ to $10 \mathrm{MHz}$. The measurement geometry was a disc shaped plate capacitor of $30 \mathrm{~mm}$ diameter where the specimen was placed between two 
gold-plated brace electrodes (Figure 4.4). The thickness of the sample was ca. $2 \mathrm{~mm}$. Thin gold layers were sputtered onto the flat surfaces of rubber specimen in order to ensure electrical contact with the electrode plates
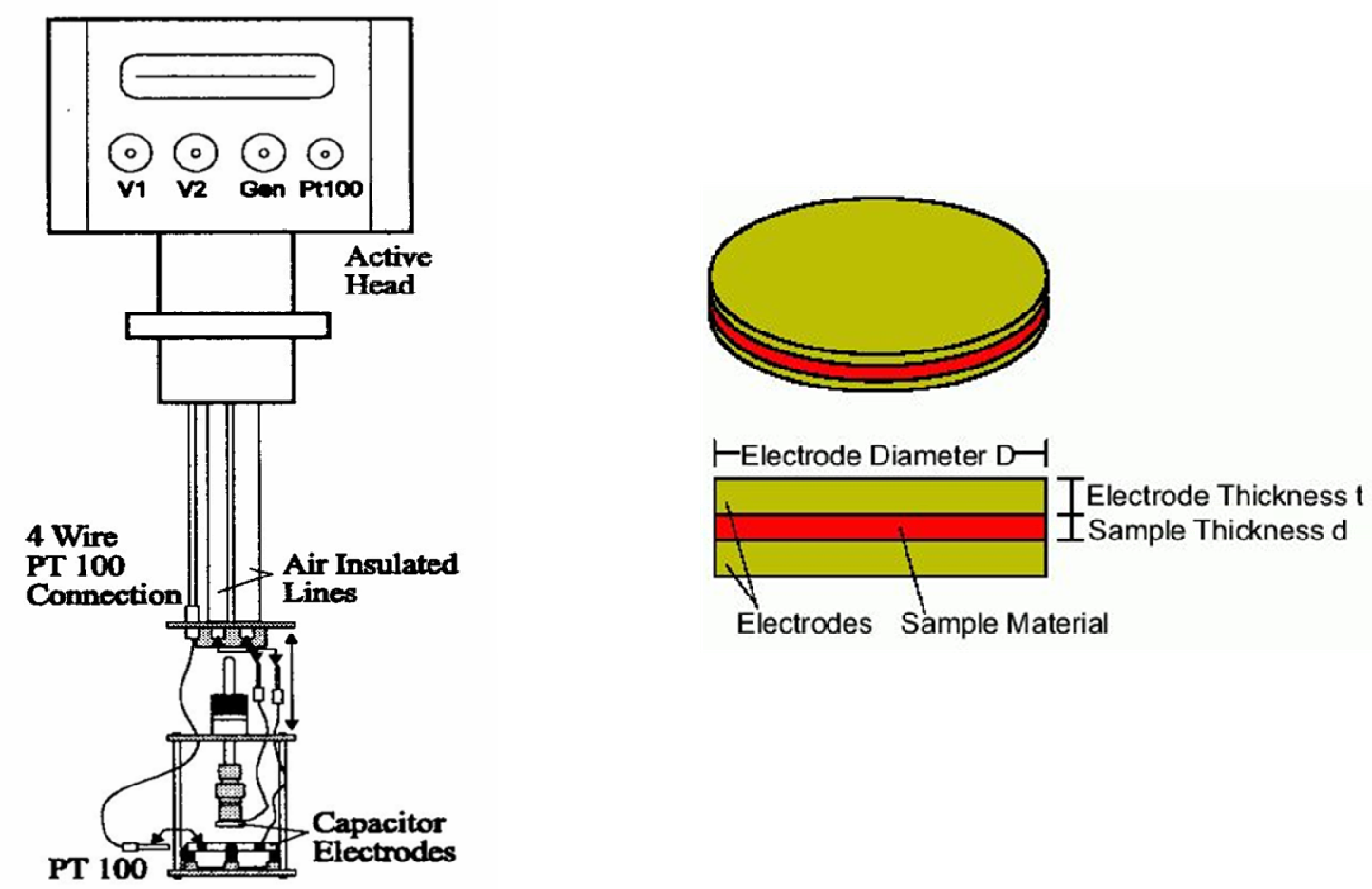

Figure 4.4: Experimental set-up of the broadband dielectric spectrometer

\subsection{Photogrammetry analysis}

The local deformation of rubber by surface asperities during sliding friction is associated with bulk deformation and related dissipative effects. In particular, the proposed formulation of the hysteresis friction assumes that the excited layer and the computed mean penetration depth vary in a linear way. In order to investigate the validity of this assumption, indentation experiments were performed with an optical measuring system ARAMIS from the company Gom. The principle is based on one or more CCD cameras that record the deformation of structure under different loading conditions (Figure 4.5). By applying a spray pattern on the surface of the specimen, facets can be detected and tracked at each stage of the loading process. The initial image processing, requiring image correlation, defines unique correlation areas known as macro-image facets, typically 5-20 pixels square which can overlap for higher accuracy across the entire image area [83]. The centre of each facet is a measurement point which is tracked during the deformation process as long as it remains in 
the field of view of the cameras. The $3 \mathrm{D}$ image correlation system uses ordinary light to reduce the glare. The minimum sensitivity is $1 / 30000$ of the field of view, e.g. a sensitivity of 1 micron can be obtained with a $3 \mathrm{~cm}$ field of view.

This leads to the reconstruction of the online deformation and stress field. Experimentally, a $10 \mathrm{~mm}$ high rubber block was glued on an aluminium plate. Two identical steel cylindrical indenters with a tip radius of $1 \mathrm{~mm}$ were used for the measurements, whereby the penetration depth was stepwise controlled with the MTS software.

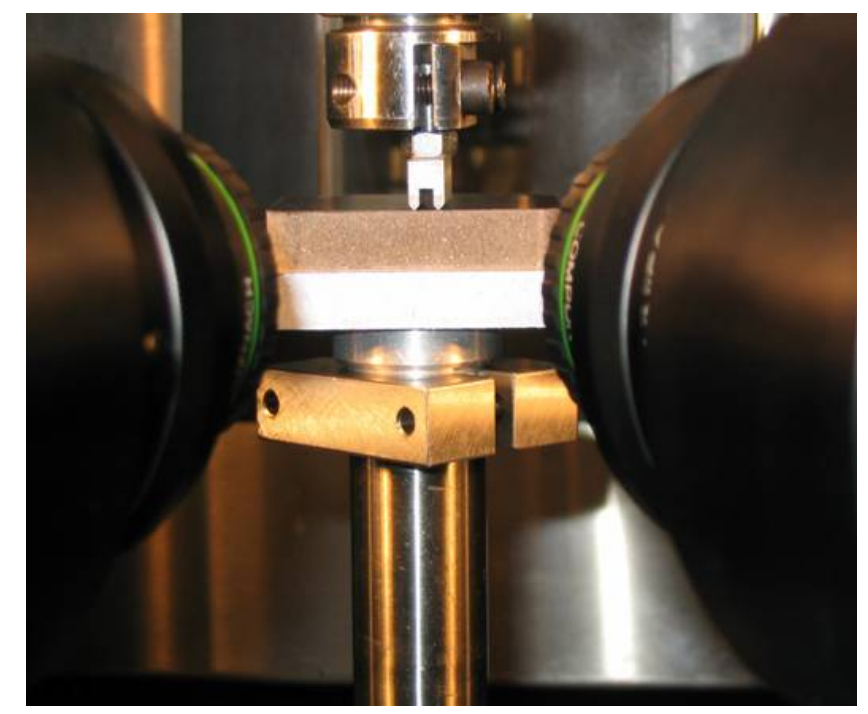

Figure 4.5: Experimental set-up of the Aramis optical measuring system

\subsection{Friction measurements}

The characterisation of the friction coefficient was performed with two different testing methods. Firstly, a Zwick universal testing machine was modified and reconfigured for friction measurements. Thus, tests could be carried out over a range of low sliding velocities (up to $\mathrm{v} \sim 10 \mathrm{~mm} / \mathrm{s})$ and moderate loads $\left(\sigma_{\circ} \sim 0.01 \mathrm{MPa}\right)$. A second possibility consisted of using an MTS biaxial servo-hydraulic testing facility. This allowed an extended range of sliding velocities (up to $v=400 \mathrm{~mm} / \mathrm{s}$ ) and loads $\left(\sigma_{\circ} \sim 0.3 \mathrm{MPa}\right.$ ).

\subsubsection{Modified Zwick universal testing equipment}

Based on a Zwick universal testing facility, stationary friction experiments could be carried out on rough surfaces under various contact conditions. It allows a characterisation of the 
friction behaviour over three decades of sliding velocity, namely from $v=0.01 \mathrm{~mm} / \mathrm{s}$ to $10 \mathrm{~mm} / \mathrm{s}$. Within this range, it can be reasonably assumed that interfacial thermal effects related to the local increase of contact temperature are negligible.

The rubber sample dimensions are $50 \times 50 \mathrm{~mm}^{2}$ with a thickness of $2 \mathrm{~mm}$. The large surface area ensures a good statistical distribution of the contact patches with the rubber surface. It was then glued to an aluminium plate loaded with weights. This ensemble is pulled by a steel rope with a diameter $d=0.54 \mathrm{~mm}$ connected to the traverse by two pulleys. One of them is fixed to the load cell where the friction force can be then directly measured. The experimental set-up of friction measurements is presented in Figure 4.6.
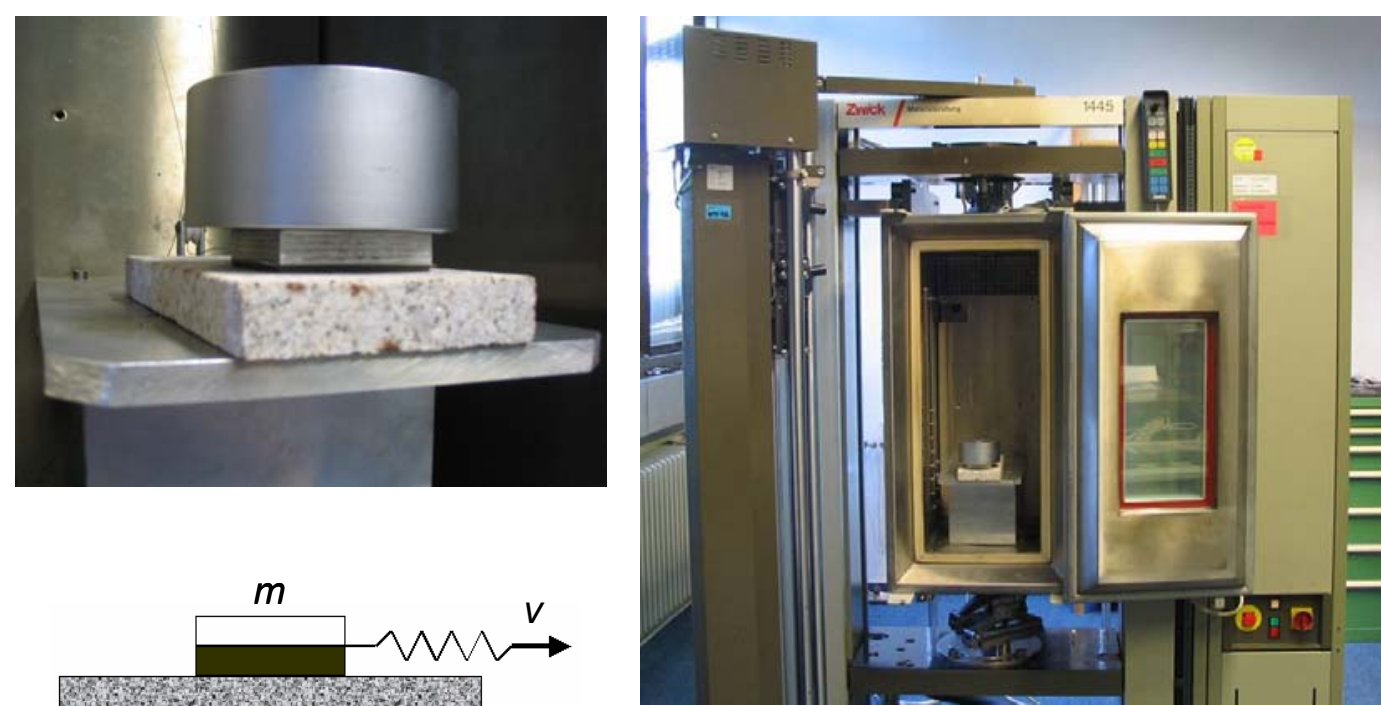

Figure 4.6: Experimental set-up for stationary friction measurements

The use of weights leads to a limited range of tested loads: it can be easily calculated that a weight of $30 \mathrm{~N}$ is, in this case, equivalent to a load $\sigma_{\circ} \sim 12 \mathrm{kPa}$. Consequently, the friction behaviour could be only investigated within a range of moderate loads. Conversely, as the amount of abrasion was significantly reduced, unfilled materials with a low hardness could be measured without a significant modification of surface texture.

Experimentally, the test was performed until the friction force reached a plateau value $F_{s t}$ characteristic of the stationary regime. The friction coefficient was then calculated as $\mu=F_{s t} / F_{N}$, where $F_{N}$ is the normal force. A typical measurement of the friction force is presented in Figure 4.7 for three different loads. 


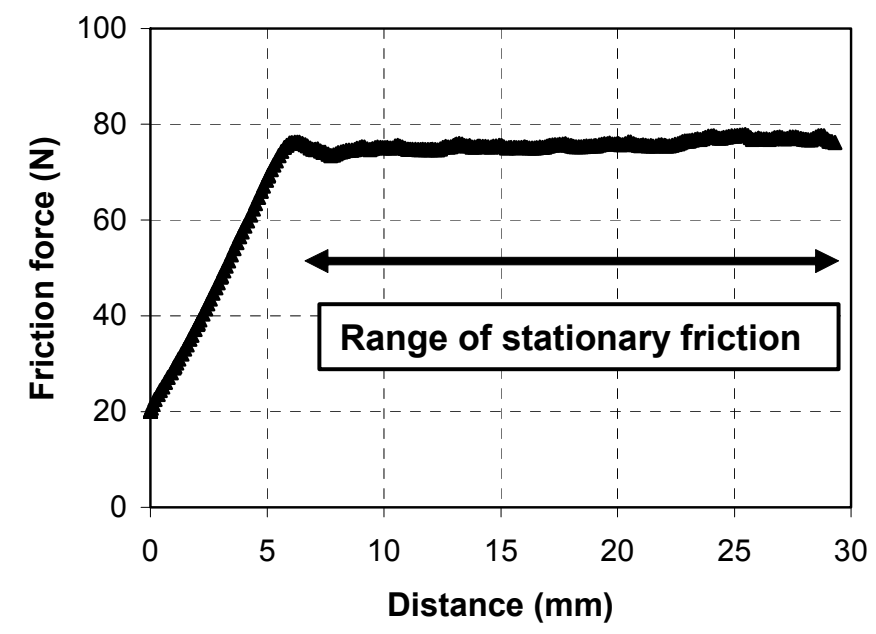

Figure 4.7: Friction force as a function of the measuring distance with corresponding stationary regime

\subsubsection{Biaxial hydropulser (MTS)}

An alternative method for friction measurements was obtained by modifying a biaxial MTS servo-hydraulic testing facility. It is equipped with two perpendicular cylinders which can apply a force up to $20 \mathrm{kN}$. Thus, classical dynamic measurements can be performed under uniaxial deformation or under complex situations of loading encountered in tyre tread elements (combination of compression and shear). The vertical axis can be moved along a linear bearing with a maximum displacement of $150 \mathrm{~mm}$. A second cylinder positioned perpendicular to the vertical axis allows horizontal movement over a range of $60 \mathrm{~mm}$. Consequently, by synchronising the displacement of both axes, a friction process can be simulated.

The construction is based on two linear bearings fixed to a bar which ensures a sufficient stiffness in the direction of translation. The rubber sample is glued on an aluminium plate mounted on the horizontal axis and has a maximal size of $60 \times 60 \mathrm{~mm}^{2}$. The vertical axis consists of a biaxial load cell rigidly attached to an aluminium bath containing a rough substrate. The biaxial load cell is based on the DMS measuring principle and measures both the normal force $F_{N}$ and the tangential force, e.g. friction force $F_{R}$, up to a maximum level of $25 \mathrm{kN}$. The aluminium form allows the possibility to use a lubricant and then carry out friction measurements under dry or wet conditions (water detergent). A detailed view of the experimental construction is shown in Figure 4.8. 

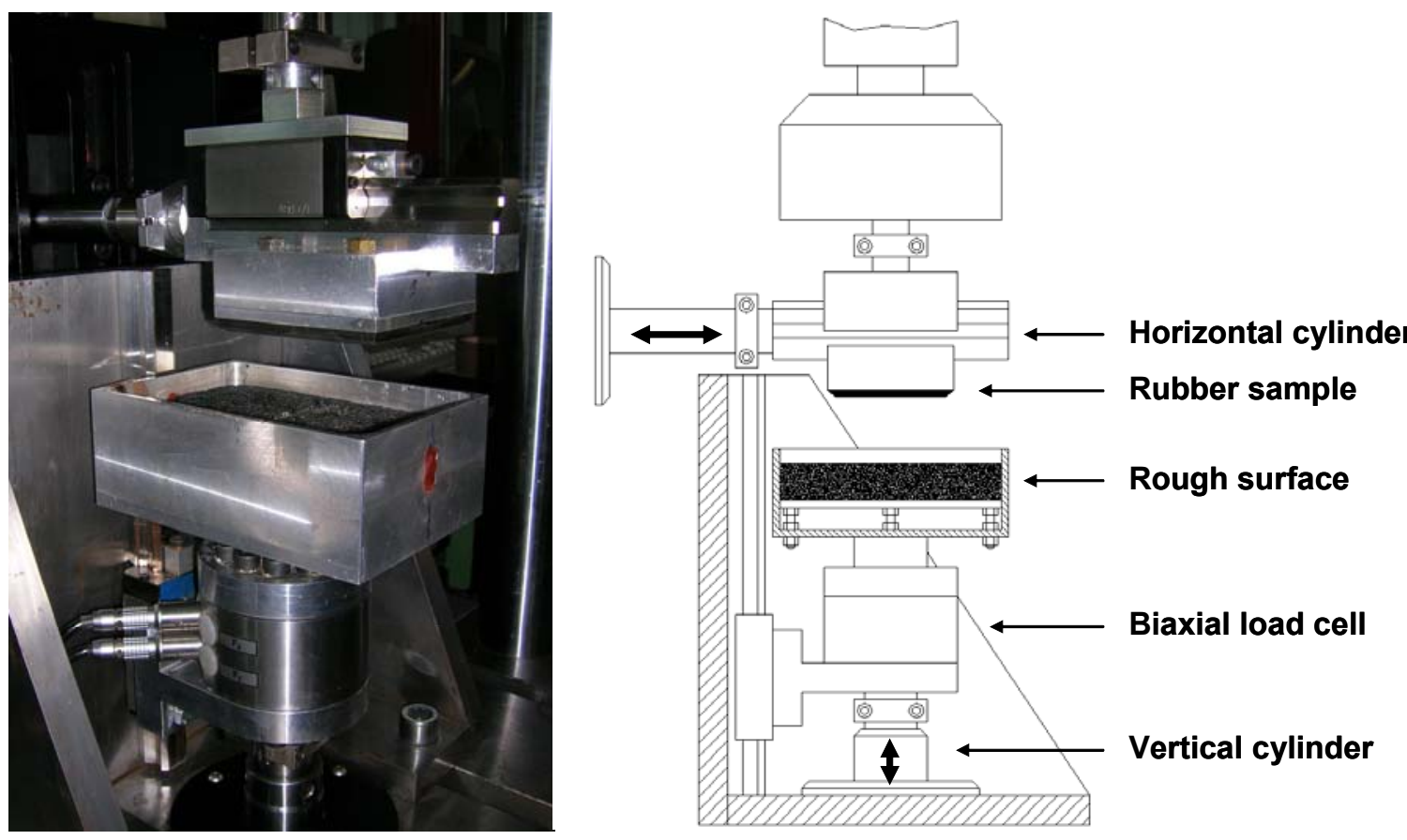

Figure 4.8: Picture of a modified biaxial MTS testing machine for friction tests (left) and schematic representation (right)

The experimental procedure was programmed on the TestWare SX module, part of the TestStar control software. Both axes were displacement controlled for friction tests: a triangular function was generated for the horizontal cylinder, thus allowing a translation of the horizontal cylinder at constant sliding velocity. The vertical axis was controlled by a rectangular signal associated with a given penetration depth of the rough substrate in the rubber material during a defined period of time. A typical example of the recorded signals is presented in Figure 4.9. It should be noted that real conditions of tread elements during ABSBraking phases can be reproduced up to the maximal sliding velocity $v=400 \mathrm{~mm} / \mathrm{s}$ by reducing the contact time (pulsed stress simulating short contact with the road) and repeating the measurement (rolling tyre). The tested sliding speeds range from $v=0.1 \mathrm{~mm} / \mathrm{s}$ to $v=300 \mathrm{~mm} / \mathrm{s}$, with a maximum friction path of $d=50 \mathrm{~mm}$. The experimental load was fixed at $\sigma_{0}=0.25 \mathrm{MPa}$. 


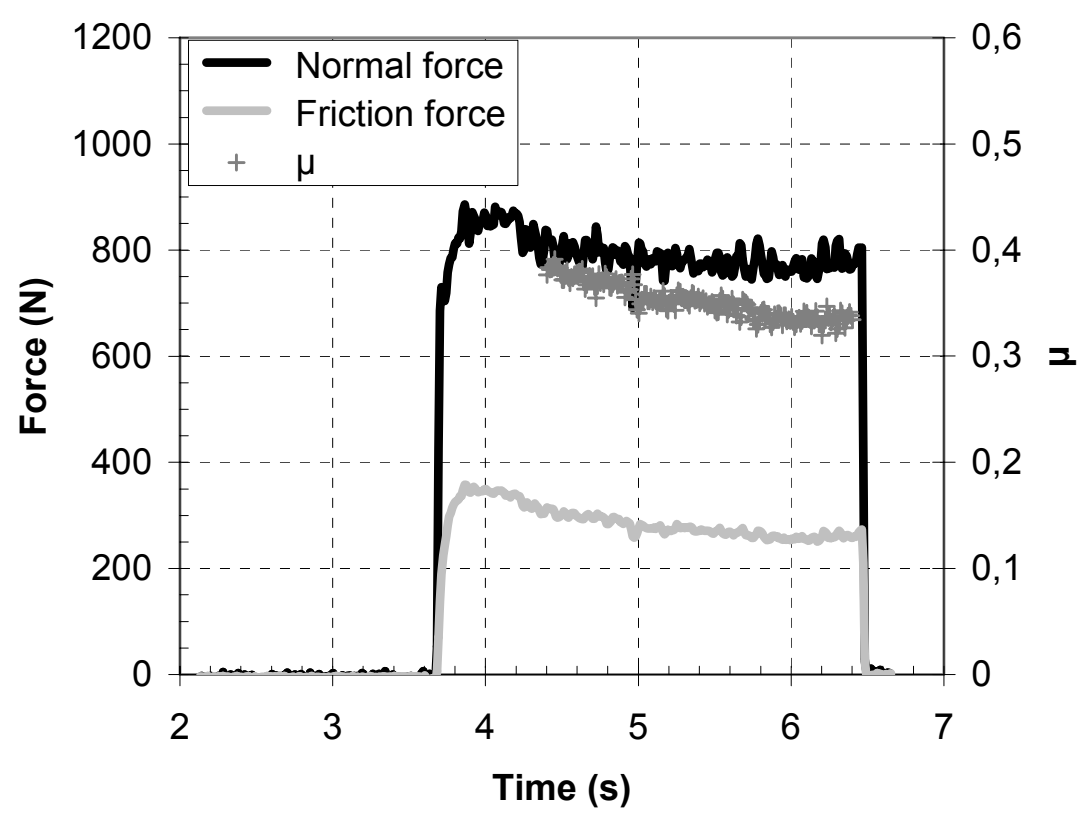

Figure 4.9: $\quad$ Measured normal and friction forces for an S-SBR 2525 filled with 60 phr N339 on a fine asphalt. $v=4 \mathrm{~mm} / \mathrm{s}$. 


\section{Experimental results and model prediction}

This chapter first presents a wide range of results required for the simulation of both hysteresis and adhesion friction. The evaluation of surface statistical functions gives a deeper insight into the scaling properties of rough substrates and highlights the relevance of a two-scaling-regimes approach for the description of roughness. The combination of relaxation spectroscopy data leads to a generalized master procedure for filled elastomers. Resulting simulations of hysteresis friction underline the influence of temperature, load, roughness, sliding velocity and rubber formulation (polymer, filler) on contact parameters during sliding process. Finally, correlations between theoretical predictions and experimental data are shown: the role of hysteresis and adhesion friction is discussed in details followed by a consequent argumentation for further improvements of the modelling.

\subsection{Roughness analysis}

The characterisation of rough surface is an essential step for the prediction of friction properties. Regarding wet grip performance, a general approach used by road constructors consists in correlating experimental data with characteristic quantities of road pavements: for instance, the standard sand height method gives an indication of the roughness amplitude and is therefore used in empirical friction laws to describe the frictional behaviour of tyres under wet conditions [80]. On the other side, tyre manufacturers often correlate the dynamic loss factor of tread compounds measured at low temperature with wet grip properties [57][58]. In both cases, friction mechanisms are assumed to arise from a characteristic, e.g. single length scale of rough profiles.

The self-affine character exhibited by road pavements has opened new perspectives for the description and comprehension of interfacial phenomena. Thereby, the roughness is considered through morphological invariance over several length scale regimes which subsequently relates to a multi-scale description of dynamic contact problems during sliding friction.

In the following, rough granite and asphalt surfaces are characterised by means of profilometry measurements. Corresponding height distributions are evaluated with regard to the Greenwood-Williamson functions required for the estimation of contact parameters. Also, the calculation of the statistical height difference correlation function $C_{z}(\lambda)$ underlines typical 
scaling behaviour of rough surfaces and leads to the determination of fractal descriptors. Finally, the influence of spatial resolution during profile measurements is schematically presented and experimental requirements for a proper characterisation of rough surfaces down to the micrometer range are pointed out.

As presented in Chapter 4, both surfaces were characterised by means of profilometry with a horizontal resolution $r=0.5 \mu \mathrm{m}$ over a maximal measuring range $\mathrm{d}=50 \mathrm{~mm}$. A typical example is shown in Figure 5.1 (left diagram) for a rough granite profile, whereby results are already averaged $(\langle z(x)>=0)$ and levelled out $(\langle\dot{z}(x)>=0)$. The maximal roughness amplitude peak-to-peak lies around $\Delta \mathrm{z}_{\max }=1 \mathrm{~mm}$, which is acceptable compared to the maximum vertical stroke of the needle. The corresponding normalized height distribution $\phi(z)$ is presented in the right diagram of Figure 5.1 together with the asphalt surface. Rough granite displays a quasi-symmetrical distribution while asphalt shows a dissymmetrical behaviour with higher roughness density over the mean value of the profile. Also the broader feature observed for the latter indicates a higher level of roughness. Both surfaces show significant advantages compared to silicon carbide based substrates which have been extensively studied during the past, namely a decrease in asperity sharpness which strongly limit the occurrence of wear during friction process [13][86].
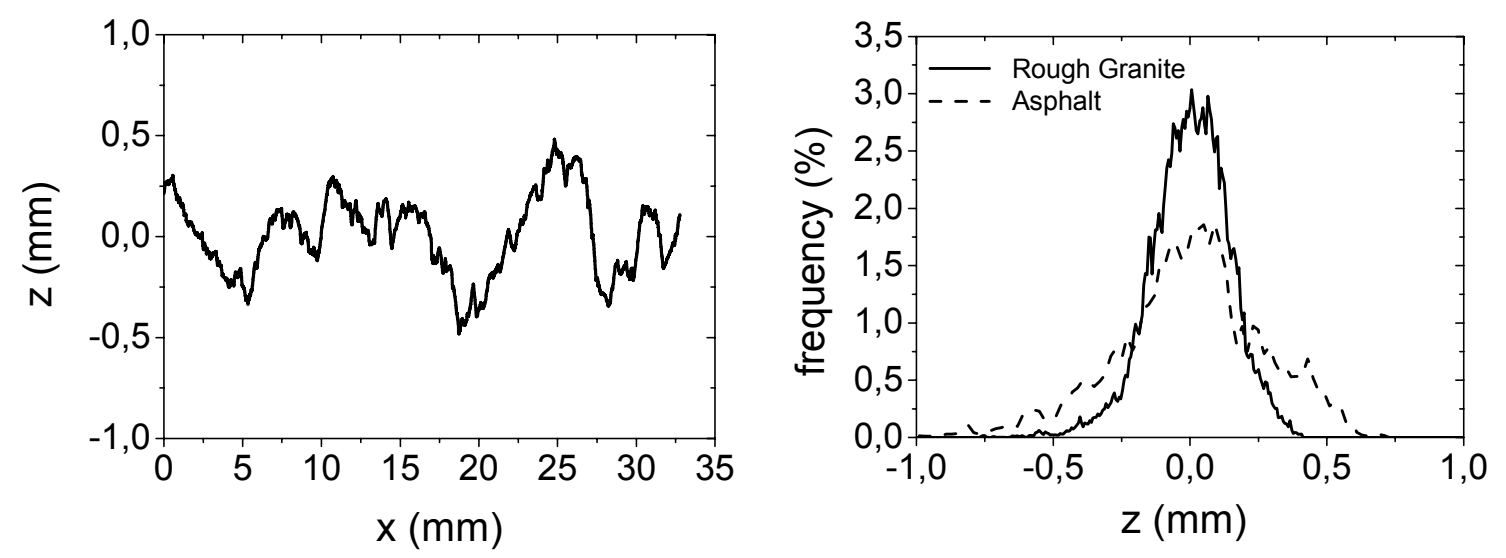

Figure 5.1: Profile measurement of rough granite (left) and associated normalized height distributions (right)

The evaluation of the height difference correlation function $C_{z}(\lambda)$ is presented in Figure 5.2 for a rough granite surface. It physically corresponds to the average height difference of two points from a profile with increasing horizontal distance. The reproducibility of numerical procedure can be seen in the left diagram of Figure 5.2: since profile measurements were carried out in different spatial directions, the roughness exhibits a quasi-isotropic feature with 
typical scaling behaviour. The averaged height difference correlation function $C_{z}(\lambda)$ is shown in the right diagram with the associated surface descriptors.
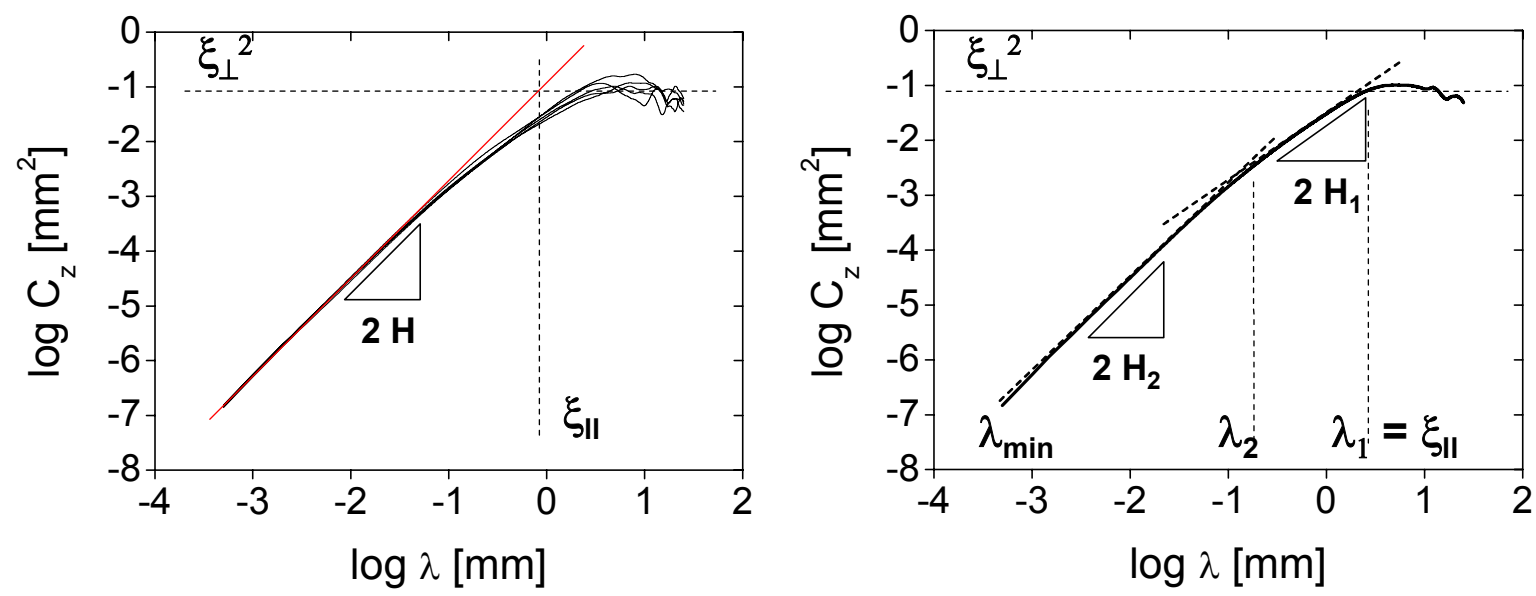

Figure 5.2: $\quad$ Height difference correlation function $C_{z}(\lambda)$ for five rough granite profiles (left) and averaged function with fractal descriptors (right)

As shown in Figure 5.2, the slope of the height-difference correlation function $C_{z}(\lambda)$, i.e. the Hurst exponent $\mathrm{H}$ or fractal dimension $\mathrm{D}$, depends on the interval of length scales selected for fitting procedure. Since the value of $D$ is known to strongly influence the simulated hysteresis friction, a special attention should be given to the interval chosen for the calculation of the fractal dimension. In practice, a full treatment of roughness requires a sufficiently high measurement range up to a few $\mathrm{cm}$ for the collection of the largest surface asperities combined with a spatial resolution down to the micrometer range for the characterisation of the smallest corrugations involved during sliding friction. For instance, roughness characterisation carried out with recent laser equipment offers wide measurement ranges up to $\lambda=40 \mathrm{~cm}$ but poorly resolves small asperities with a horizontal resolution $r=25 \mu \mathrm{m}[71][73]$. As a result, while large length scales are properly characterised, this type of measurement only provides limited information regarding the morphology of small length scales which are crucial for the modelling and occurrence of adhesion processes [66].

Based on results obtained for rough granite, a systematic variation of the interval of evaluation was carried out in order to quantify the scatter of $D$ values within the micrometer range. Results show a gradual increase of $D$ when larger length scales are included in the fitting procedure (Table 5.1). Since the horizontal resolution of the needle measuring equipment is $r=0.5 \mu \mathrm{m}$, the calculation procedure appears to be critical within this range due to a lack a precision. Therefore, a safety margin should be taken to ensure the validity of the evaluation. On the other side, the scaling behaviour can be graphically observed up to 200 
$\mu \mathrm{m}$ (Figure 5.2). By restricting the evaluation interval to [25 $\mu \mathrm{m}-200 \mu \mathrm{m}$ ] like it would be the case for a laser measurement, the fractal dimension is found to be $D=2.23$. This confirms the fact that inherent limitations of profilometry equipments lead to unsatisfying description of micro-roughness which can in return mislead the prediction of hysteresis and adhesion friction.

\begin{tabular}{cc}
\hline Interval $\left[\lambda_{2}, \lambda_{1}\right][\mu \mathrm{m}]$ & Fractal Dimension D \\
\hline$[0.5-50]$ & 2.12 \\
{$[0.5-200]$} & 2.18 \\
{$[5-100]$} & 2.14 \\
{$[5-200]$} & 2.20 \\
{$[25-200]$} & 2.23 \\
\hline
\end{tabular}

Table 5.1: Fractal dimension $D$ of microtexture calculated within different ranges $\left[\lambda_{2}, \lambda_{1}\right]$ for a rough granite surface

As previously observed, a scaling regime can be identified at length scales comprised between the interval $\lambda=[5-100 \mu \mathrm{m}]$ with a corresponding fractal dimension $D_{2}=2.14$. For length scales larger than $\lambda \sim 100 \mu \mathrm{m}$, the path of the height difference correlation function strongly deviates from the formulated assumption which indicates the existence of a second scaling regime up to the largest length scales. By applying a fitting procedure on the upper interval $\lambda=[200-1000 \mu \mathrm{m}]$, a second fractal dimension $D_{1}=2.37$ can be determined. Compared to the former description with one scaling regime, the horizontal cut-off length $\xi_{\|}$is shifted towards higher values due to the higher fractal dimension of macro-roughness (from $\xi_{\|}=1.1$ to $2.5 \mathrm{~mm}$ ). The impact of $\xi_{\|}$on contact parameters is shown and explained in the next section. Also, a third cut-off length $\lambda_{2}$ can be defined, corresponding to the intersection point of scaling ranges, namely the limit between micro- and macrotexture. Up to now, no precise definition of both micro- and macrotextures in terms of length scales is available in the literature. Nevertheless, their common limit is generally expected to be located around a few hundred microns, thus corroborating results found on rough granite [5]. Referring to the modelling of hysteresis friction, the length scale $\lambda_{2}$ is associated with the boundary frequency $f=v / \lambda_{2}$ which delimitates both friction integrals in case of a two-scaling-regimes approach.

Similarly to rough granite, asphalt profile measurements have been evaluated on the basis of statistical correlation functions. The corresponding height difference correlation function $C_{z}(\lambda)$ and surface descriptors are depicted in Figure 5.3. Fractal analysis reveals the existence of two scaling regimes associated with distinct fractal dimensions. The microtexture is found to 
be related to a fractal dimension $D_{2}=2.08$ within the interval $\lambda=[5-100 \mu \mathrm{m}]$ and a second scaling regime can be identified with $D_{1}=2.44$ within the range $\lambda=[300-1000 \mu \mathrm{m}]$ which is associated with a cut-off length $\lambda_{2}=280 \mu \mathrm{m}$.
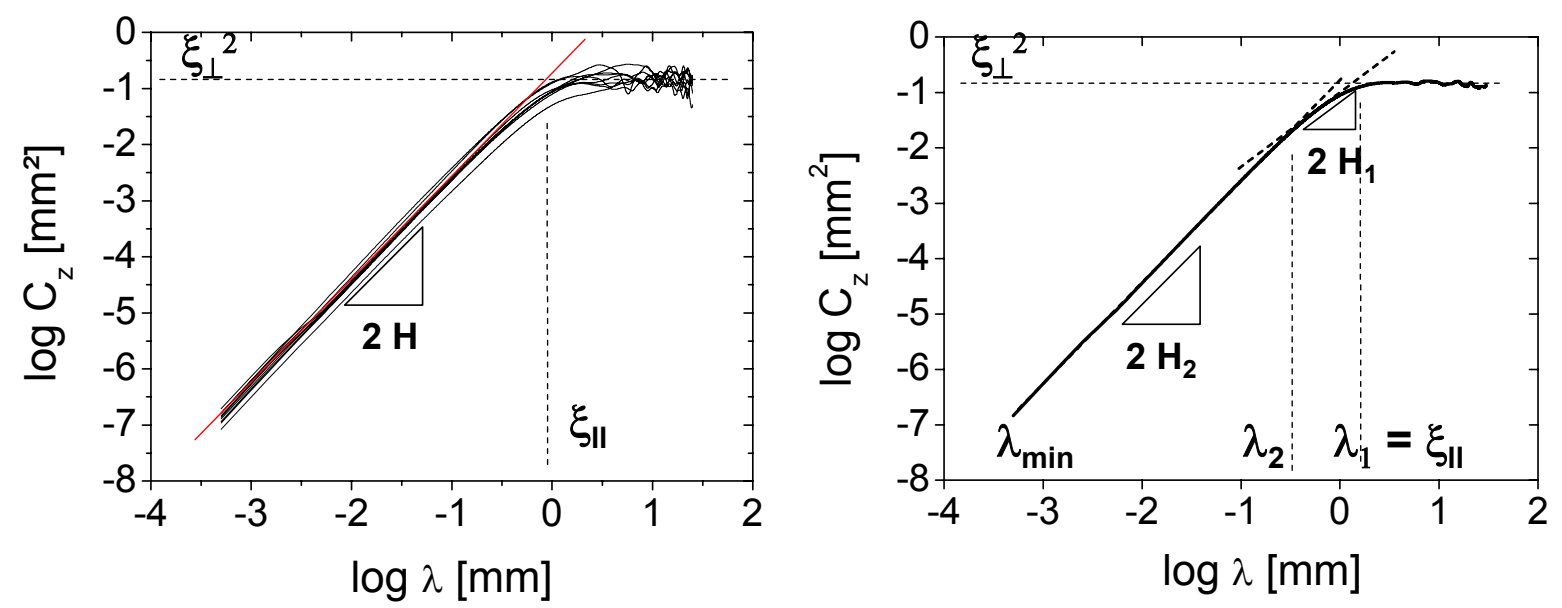

Figure 5.3: $\quad$ Height difference correlation function $C_{z}(\lambda)$ for ten asphalt profiles (left) and averaged function with fractal descriptors (right)

The small interval of the second scaling regime can be traced back to the nature of asphalt surfaces: indeed, the morphology of macrotexture is mainly determined by the grain size distribution, which in this case exhibits a narrow feature. Comparatively, the rough granite was fractured and processed by a manual finishing which leads to a stronger dependence of the morphology upon length scale.

During the dynamic contact between elastomers and rough surfaces, the normal force is statistically distributed over the largest asperities of the substrate. The contribution of roughness at smaller length scales on the mean push-in of the rubber is negligible, e.g. the load is a quantity which does not depend on the length scale at which the contact is observed. This is expressed by Equation (3.14), where a summit height distribution of macroscopic summits $\phi_{s}(z)$ is introduced to describe the upper part of the profile which is actually in contact with the rubber. In order to fulfil basic assumptions of Hertz theory, a procedure for the determination of $\phi_{s}(z)$ has been presented in the theoretical section and further on successfully applied to a silicon carbide surface. The method relies on a numerical algorithm which seeks local maxima over different interval lengths in combination with an analytical affine transformation. Since the summit height distribution corresponds to the largest length scales of profile, the interval length is given by $\lambda=\xi_{\| l}$, which corresponds to the diameter of 
spheres the rough profile is approximated with. As a result, a surface specific affine parameter $\mathrm{s}$ is determined and implemented in the hysteresis friction model.

Table 5.2 summarizes surface descriptors with associated affine parameters obtained for rough granite and asphalt with a one and two-scaling-ranges description of roughness. It should be noted that the position of the horizontal cut-off length is shifted whether the roughness is described by one or two scaling regimes - corresponding to a larger interval length for the distribution of maxima - and therefore affects the affine parameter s. The affinelike transformed profile was superimposed according to the mean value of the numerical height distribution, which leads to $s=1.25$ for rough granite with a one-scaling-regime approach. In a similar way, the procedure was applied to asphalt and gives $s=1.23$ (one scaling range). By describing the height difference correlation function with two scaling ranges, a slight increase of the affine parameter is observed so that one obtains $s=1.27$ and 1.24 for the rough granite and asphalt, respectively.

\begin{tabular}{ccccc}
\hline & \multicolumn{2}{c}{ Rough Granite } & \multicolumn{2}{c}{ Asphalt } \\
& 1 S.R. & 2 S.R. & 1 S.R. & 2 S.R. \\
\hline $\mathrm{D}_{2}[5-100 \mu \mathrm{m}]$ & 2.14 & 2.14 & 2.08 & 2.08 \\
$\mathrm{D}_{1}[300-1000 \mu \mathrm{m}]$ & $\mathrm{x}$ & 2.37 & $\mathrm{x}$ & 2.44 \\
$\xi_{\perp}[\mathrm{mm}]$ & 0.31 & 0.31 & 0.38 & 0.38 \\
$\xi_{\|}[\mathrm{mm}]$ & 1.06 & 2.49 & 0.90 & 1.55 \\
$\lambda_{2}[\mathrm{~mm}]$ & $\mathrm{X}$ & 0.093 & $\mathrm{x}$ & 0.28 \\
s parameter & 1.25 & 1.27 & 1.23 & 1.24 \\
\hline
\end{tabular}

Table 5.2: Surface descriptors and affine parameters for rough granite and asphalt with a one and two-scaling-ranges approach

From gained height distributions, Greenwood-Williamson functions can be calculated according to Equation (3.13), which further on gives access to contact parameters like the mean penetration depth $\left\langle z_{p}\right\rangle$. In particular, $F_{0}(t)$ displays the probability that contact occurs above $t=d / \tilde{\sigma}$, where $\mathrm{d}$ is the distance rubber - mean value of rough profile. Figure 5.4 shows the Greenwood-Williamson functions for rough granite and asphalt surfaces as a function of the normalized distance. Contrary to $F_{0}$ and $F_{1}, F_{3 / 2}$ is related to the summit height distribution $\phi_{s}(z)$ and its standard deviation $\tilde{\sigma}_{S}$, whereby the relationship between $t$ and $t_{s}$ is given by Equation (3.15) and (3.16). Beyond the relevance of Greenwood-Williamson functions for contact mechanics, their implication on the load dependence of hysteresis friction coefficient will be shown later in this section. 

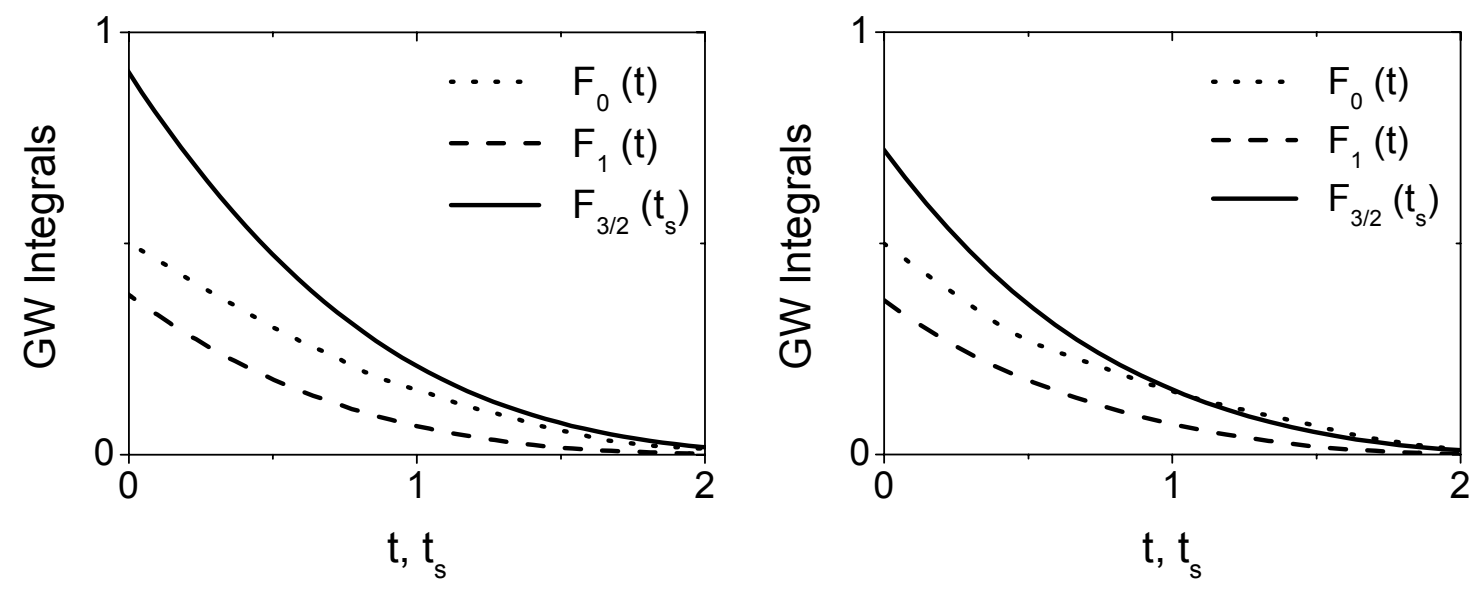

Figure 5.4: Greenwood-Williamson functions of rough granite (left) and asphalt (right)

\subsection{Contact analysis}

Contact parameters play a crucial role during sliding processes on rough surfaces as they determine the deformation of the bulk rubber, e.g. hysteresis friction, and the amount of contact patches available to generate shear stresses, namely the adhesion component. In particular, the modelling of hysteresis friction relies on a proportional relationship between the excitated layer $\langle\delta\rangle$ and the mean penetration depth $\left\langle z_{p}\right\rangle$. In order to investigate this assumption, indentation experiments have been carried out under static conditions on a thick rubber pad locally compressed by indenters with a tip radius $R=1 \mathrm{~mm}$. Through the combination of MTS testing equipment and photogrammetry technique, the strain field could be measured and characterised at different stages of the indentation process. The displacement of the indenter, e.g. the maximum penetration depth, was stepwise increased during the experiment, while a characteristic picture of the deformation state was recorded and further on analyzed by the photogrammetry software.

As an example, Figure 5.5 shows the spatial reconstruction of strain field at a penetration depth $z_{p}=0.2 \mathrm{~mm}$ for a carbon black filled S-SBR 5025. As expected, the deformation process is observed in the bulk material with a strongly inhomogeneous ellipsoid-like strain field going up to a distance $d=10 \mathrm{~mm}$ from the reference non deformed surface. Although the optical resolution limits the quality of results close to the surface, maximum strains of at least $\varepsilon=20 \%$ are reached at the vicinity of the indenter. The pronounced strain gradient associated with contact stresses raises the question of the strain amplitude used for the simulation of hysteresis friction. Due to the well-known strain dependence of dynamic properties for filled elastomers, one expects different predictions of hysteresis friction 
whether the simulation is performed at low and high strain amplitudes. In addition, experimental parameters - normal force, sliding velocity, temperature - should influence the dynamic indentation behaviour of elastomers on rough surfaces. A simplification of indentation mechanisms for rubbery materials consists in simulating the hysteresis friction at high strain amplitudes, whereby non-linear effects are considered and averaged over the excitated volume. The impact of such an approach is highlighted in the next section.

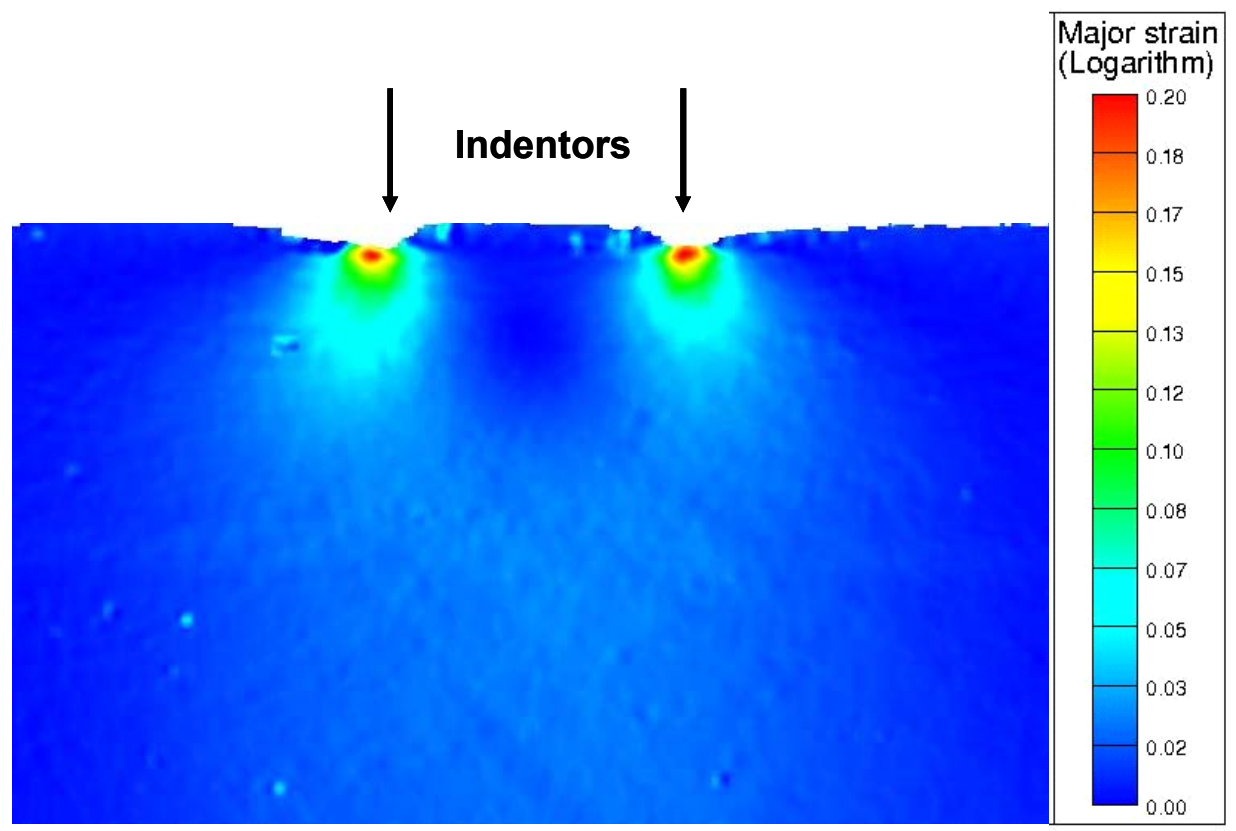

Figure 5.5: Measurement of strain field by photogrammetry technique under static conditions. Material: S-SBR 5025 / 60phr N339

Regarding contact parameters and the assumption formulated on the hysteresis friction, the excitated layer $\left\langle\delta>\right.$ and the mean penetration depth $\left\langle z_{p}>\right.$ were calculated at different stages during the indentation process. While the penetration depth is given by the geometry and displacement of the indenter, the excitated layer requires the introduction of a criterion to partition the deformed region. This can be achieved by defining a minimum strain amplitude $\varepsilon_{\min }$ above which the deformation is considered in the calculation procedure of $<\delta>$ (Figure 5.6). Consequently, the excitated layer varies with the inverse of $\varepsilon_{\min }$. Results show that a linear behaviour is observed between both quantities independently of the criterion chosen for the strain amplitude. The amplitude of the factor $b=\langle\delta\rangle /\left\langle z_{p}\right\rangle$ appears to be strongly influenced by the choice of $\varepsilon_{\min }$, and is found to vary between $\mathrm{b}=15$ and 4.5 for the current experiments. This result confirms the assumption made in Chapter 3 and suggests a strong relationship between the ratio $\left\langle\delta>/\left\langle z_{p}\right\rangle\right.$ which scales the hysteresis friction and the strain amplitude used in the simulation procedure. Indeed, low strain amplitudes encompass the quasi-totality of the deformation field and are therefore 
associated with high $b$ values, while the consideration of the high strain region spatially restricts the deformed region and thus the $b$ factor.

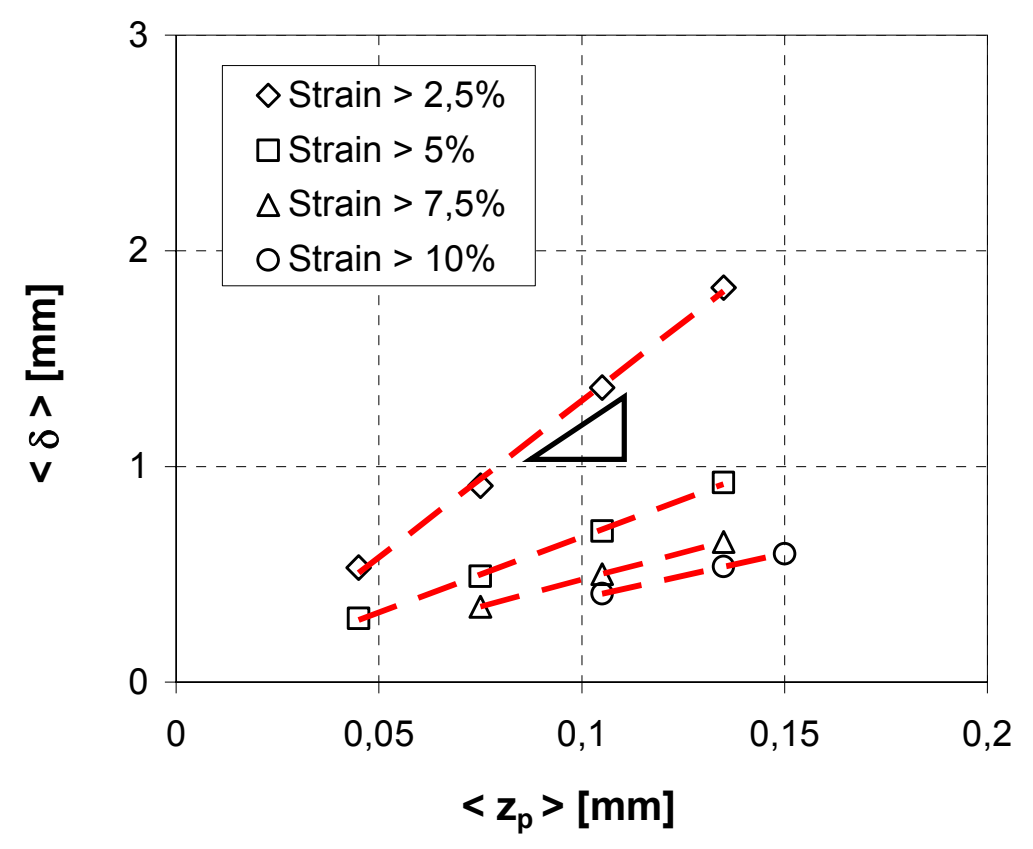

Figure 5.6: Estimated deformed layer as a function of the mean penetration depth. The slope displays the ratio $b=\left\langle\delta>/\left\langle z_{p}\right\rangle[68]\right.$

\subsection{Relaxation spectroscopy}

In the following, based on a combination of dielectric and dynamic mechanical data, a generalized master procedure is proposed for filler elastomers. Thermally activated processes at the vicinity of filler particles above glass transition appear to be determining for the overlapping of dynamic moduli in the low frequency region. The corresponding activation energy values are physically discussed on the basis of microstructure.

\subsubsection{Dielectric analysis}

Dielectric analysis consists in characterising the interaction between a given material and dynamic electrical fields. When a material is subjected to an electrical field, two mechanisms can be detected: first a reversible storage of the field energy denoted as polarization (capacitive effect), and secondly an irreversible dissipation known as conductivity (resistive effect). For polymer composites, the occurrence of polarization is related to the existence of dipole moments along polymer chains, whose strength basically depends on molecular architecture. The orientation of dipole moments involves motions of molecules and segments 
of molecules in the material. Subsequently the spectrum of retardation times arising from polarization mechanisms at different length scales is distributed over many frequency decades.

Through novel measuring techniques, a broad range of temperature and frequency is experimentally accessible during dielectric analysis. This allows a full characterisation of relaxation processes in the temperature and frequency domain without use of extension techniques the like time-temperature superposition principle introduced for dynamic mechanical analysis. The experimental coverage of the high frequency range makes dielectric analysis a powerful method for the direct detection of short-time relaxation processes, for instance local chain segment dynamics at the interface polymer-filler which otherwise can not be observed during dynamic mechanical spectroscopy.

Compared to thermoplastics, rubbery materials exhibit a soft state at room temperature, which in return means that the glass transition occurs at low temperatures. A 3D plot of the dielectric loss $\varepsilon "$ is shown in Figure 5.7 for an unfilled S-SBR 5025 as a function of temperature and frequency. Isothermal measurements were carried out within the frequency range $f=\left[10^{-2}-10^{6} \mathrm{~Hz}\right]$ at temperatures comprised between $\mathrm{T}=-60^{\circ} \mathrm{C}$ and $100^{\circ} \mathrm{C}$. Hereby, the glass transition process is clearly indicated by the dielectric loss peak occurring over the whole range of frequency. Since the material is unfilled, no additional relaxation process is found below the glass transition temperature. On the other side, the sharp increase of the dielectric loss at high temperatures indicates that the material becomes electrically conductive in the rubbery state. A typical feature of polymeric material can be seen in Figure 5.7 , namely the shift of the glass transition process with increasing frequency. This can be summarized on a so-called retardation map in which the frequency associated with the dielectric loss maximum is plotted as a function of the reciprocal temperature (Figure 5.9). The temperature dependence of the dielectric loss peak is typically described by the VFT semi-empirical equation - or equivalently the WLF approach - which characterises cooperative molecular motions within the glass transition regime. The characterisation of polymer dynamics by means of dielectric spectroscopy is a fundamental tool for questioning the applicability of master procedure during dynamic mechanical analysis.

The sole addition of filler in a given polymer matrix leads to a significant change of the dielectric feature. In particular, electrically conducting fillers like carbon black tend to form a percolating network at sufficiently high volume content, resulting in a high level of dielectric loss over the whole experimental range. The resolution of polymer dynamic is thus hindered by this effect. 


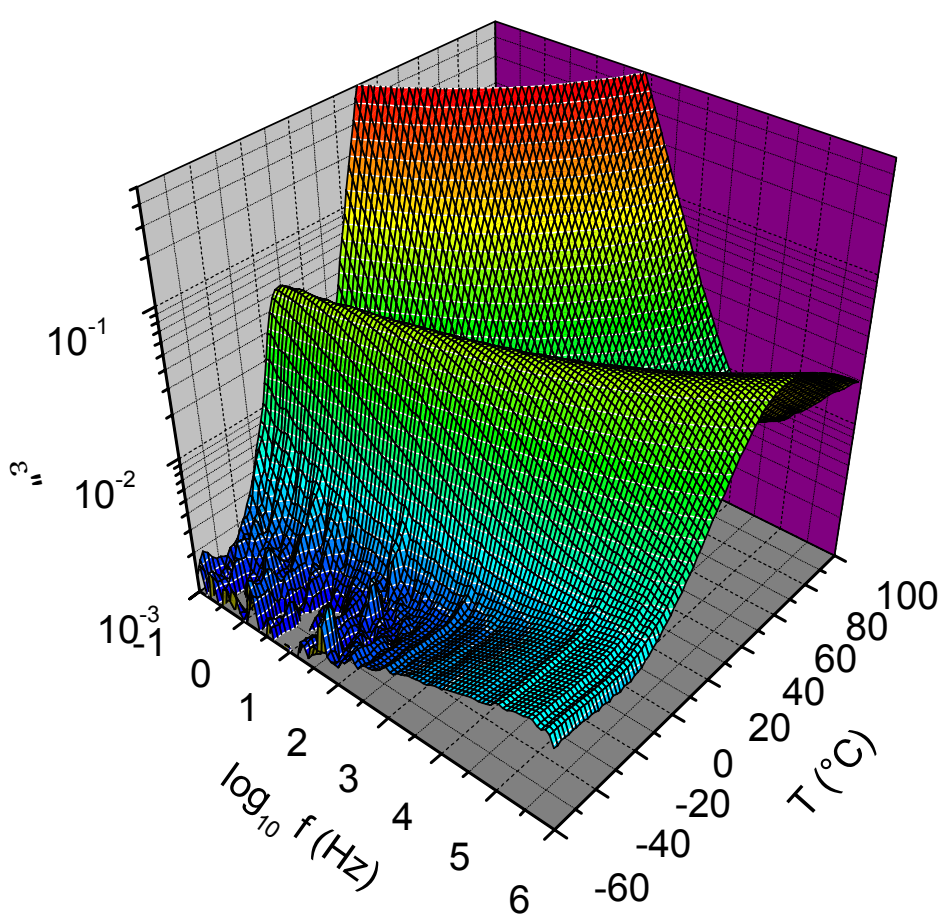

Figure 5.7: Measurement of the temperature and frequency dependent dielectric loss $\varepsilon "(\omega, T)$ for an unfilled S-SBR 5025

However, the incorporation of non-conducting fillers such as silica still allows a full characterisation of dielectric properties and a priori the detection of relaxation processes. A dielectric measurement is shown in Figure 5.8 for the same basis polymer previously characterised in Figure 5.7, namely S-SBR 5025, filled with 60phr silica. It should be kept in mind that both silica filled S-SBR grades used in this study include a coupling agent which ensures a high level of dispersion and also chemically bonds filler particles to the polymer matrix. Previous features observed on the unfilled composite can also be identified in the presence of silica: the glass transition process can be clearly detected and the material becomes electrically conductive at high temperatures. Below the glass transition, two effects are worth being noticed: first, due to the addition of filler, the level of dielectric loss is globally higher. Secondly, at very low temperatures, a second relaxation peak can be observed, denoted as silica peak. The silica peak has been found to be directly related to the presence of filler in the polymer matrix. In particular, the retardation map with different silica content and furthermore with variable interfaces shows that the low temperature peak can be described by an Arrhenius relationship [85]. This indicates that the polarization effect arises from local interfacial mechanisms between silica particles and polymer chains. Thereby, the nature of the interactions silica/polymer, namely attractive or non-attractive, will result either in slowed down or paced up local dynamic, respectively. 


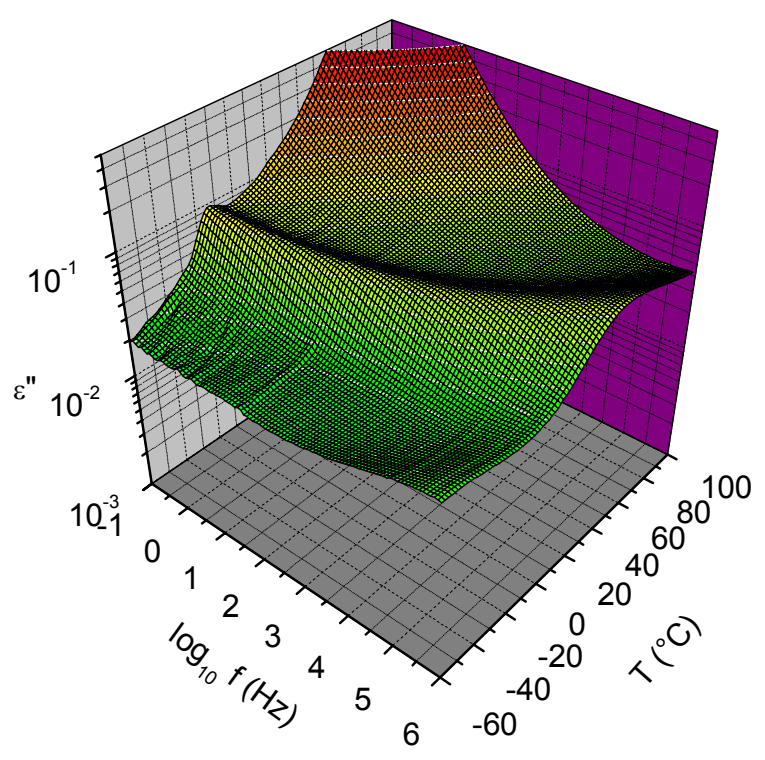

Figure 5.8: Measurement of the temperature and frequency dependent dielectric loss $\varepsilon^{\prime \prime}(\omega, T)$ for a filled S-SBR 5025 with $60 \mathrm{phr}$ silica

The examination of the Vogel-Fulcher functions gives insight into polymer dynamics for elastomers. In particular, one question arising from the dielectric analysis regarding dynamic properties of filled elastomers is: how far does the introduction of filler particles affect the polymer dynamic? By looking at the retardation maps of silica filled S-SBRs, it is found that an increasing filler content has a negligible influence on the location of the glass transition temperature characterised by the dielectric loss peak (Figure 5.9).

In other terms, the polymer dynamics is a characteristic of the bulk material while interfacial effects at the vicinity of filler particles only occur in the glassy state at very low temperatures - or high frequencies. A similar analysis was carried out on S-SBR basis polymer with $25 \%$ vinyl content (see Figure 8.1 and Figure 8.2 in Appendix). The lower vinyl content results in a shift of the glass transition temperature of about $\Delta \mathrm{T}=20^{\circ} \mathrm{C}$, as shown in Figure 8.4. The addition of silica has no significant impact on the evaluated retardation maps, and a fortiori on the WLF semi-empirical functions. This confirms previous observations made on the high$\mathrm{T}_{\mathrm{g}}$ S-SBR 5025 polymer, whereby the main relaxation process is found to solely display the contribution of the bulk material, namely the polymer matrix.

This result has strong implications on the master procedure performed during dynamic mechanical analysis. Indeed, since the WLF semi-empirical functions are not affected by the addition of filler for a given basis polymer, the same set of horizontal shift factors should be applied to the dynamic moduli above the glass transition temperature up to maximal 
temperature $\mathrm{T}=\mathrm{T}_{\mathrm{g}}+100^{\circ} \mathrm{C}$. Further consequences on the master procedure for filled compounds will be discussed in the following section.
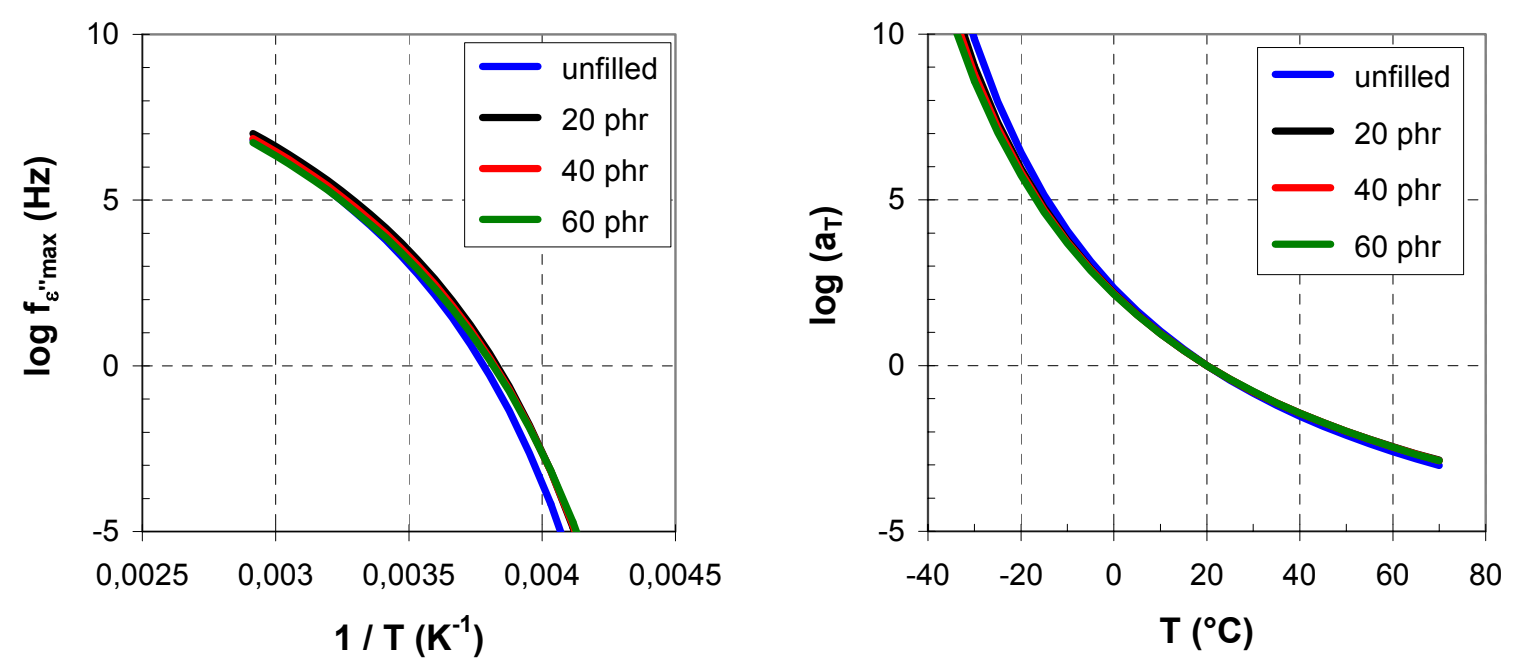

Figure 5.9: Activation Plots for silica filled S-SBR 5025 (left) and associated WLF functions (right)

\subsubsection{Dynamic mechanical analysis}

Beside the dielectric analysis, the relaxation behaviour of elastomers was investigated by means of dynamic mechanical measurements. In this case, a rubber specimen is subjected to a shear equivalent deformation within a defined range of angular frequency $\omega=[0.1-100 \mathrm{rad} / \mathrm{s}]$ at various temperatures. By referring to the time-temperature superposition principle introduced in Chapter 2, the high-frequency behaviour of elastomers can be extrapolated and the so-called master curves are constructed. The concepts of free volume and chain mobility yield as physical background for the master procedure in which the emphasis is put on the ability for a polymer chains to rearrange within a shorter time with increasing temperature [8][9]. In that way, the limited range of frequency experimentally accessible by mechanical spectroscopy methods can be extended to several decades.

The dynamic mechanical analysis has been primarily performed on unfilled systems which are suitable for basic investigations due to the negligible dependence of their dynamic properties with respect to the strain amplitude. Unfilled compounds were systematically shifted according to the loss factor tan $\delta$ (Figure 2.7). Since this quantity does not depend on the sample geometry, it solely represents a material characteristic. The resulting frequency dependent dynamic moduli are shown in Figure 5.10 for an unfilled S-SBR 5025 at a reference temperature $T_{\text {ref }}=20^{\circ} \mathrm{C}$. The curve exhibits typical features of unfilled elastomers, 
namely a low dissipative component at low frequencies ( $G^{\prime \prime}<<$ '), and a pronounced tan $\delta$ peak in the glass transition region $(\tan \delta>1)$.

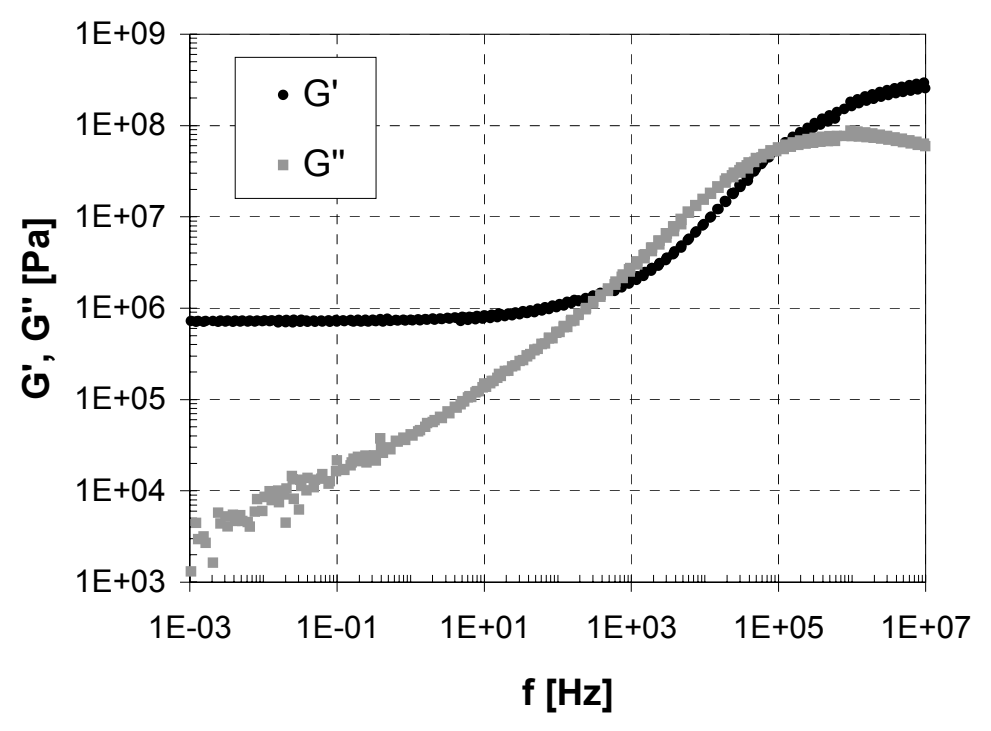

Figure 5.10: Master curve of dynamic storage (G') and loss (G') moduli for an unfilled SSBR $5025\left(T_{\text {ref }}=20^{\circ} \mathrm{C}, \varepsilon=0.5 \%\right)$

The corresponding horizontal shift factors are plotted in Figure 5.11 as a function of the temperature. Comparatively, dielectric results previously determined via Vogel-FulcherTammann functions in Figure 5.9 are superimposed. A good agreement is observed between both relaxation spectroscopy methods, which indicate that they succeed in characterising the polymer dynamics within the glass transition regime.

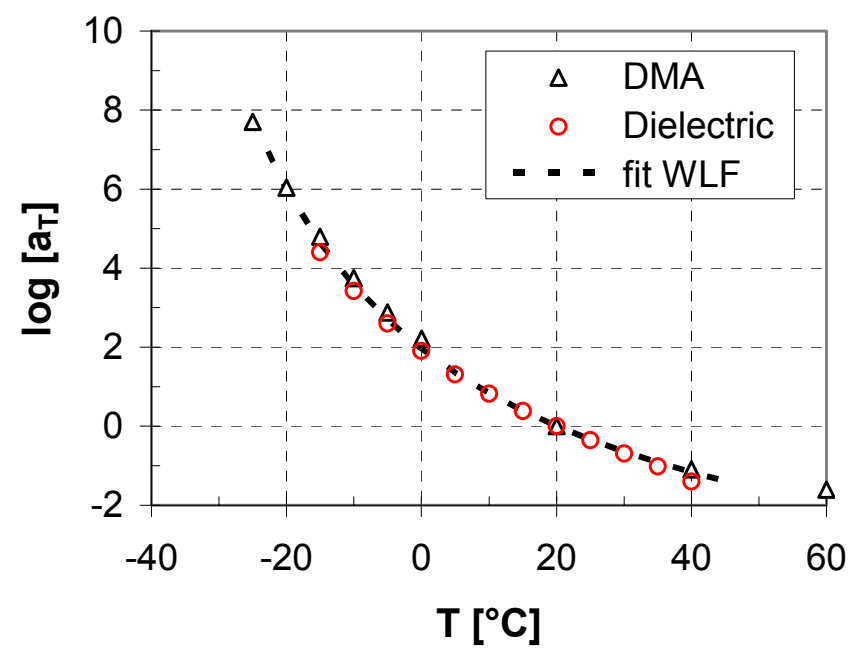

Figure 5.11: Comparison between horizontal shift factors obtained via dielectric functions and manual horizontal shift factors determined by mechanical spectroscopy for an unfilled S-SBR 5025 
As shown in the previous section, the addition of filler in the polymer matrix has no significant influence on the main relaxation process detected during relaxation spectroscopy. Therefore, horizontal shift factors used during dynamic mechanical analysis should not be affected by the filler content, e.g. the master procedure - through horizontal shift factors - should be applied in the same way whether the elastomer is filled or not. Figure 5.12 shows the example of carbon black filled S-SBR 5025 dynamically characterised at a low strain amplitude $\varepsilon=0.5 \%$ in order to avoid the occurrence of non-linear effects. As a result, the dynamic glass transition - illustrated by the loss modulus peak - can be identified and located at frequency around $f=100 \mathrm{kHz}$ at a reference temperature $\mathrm{T}=20^{\circ} \mathrm{C}$. On the other side, the range of low frequency is characterised by a poor overlapping for both storage and loss moduli components. This range practically corresponds to the frequency sweeps carried out at high temperatures, namely $\mathrm{T}=20,40$ and $60^{\circ} \mathrm{C}$. Also, the discrepancy appears to be more pronounced for the loss component of dynamic modulus. Consequently, the effect appears to be directly related to an impact of the filler on dynamic properties above the glass transition temperature.

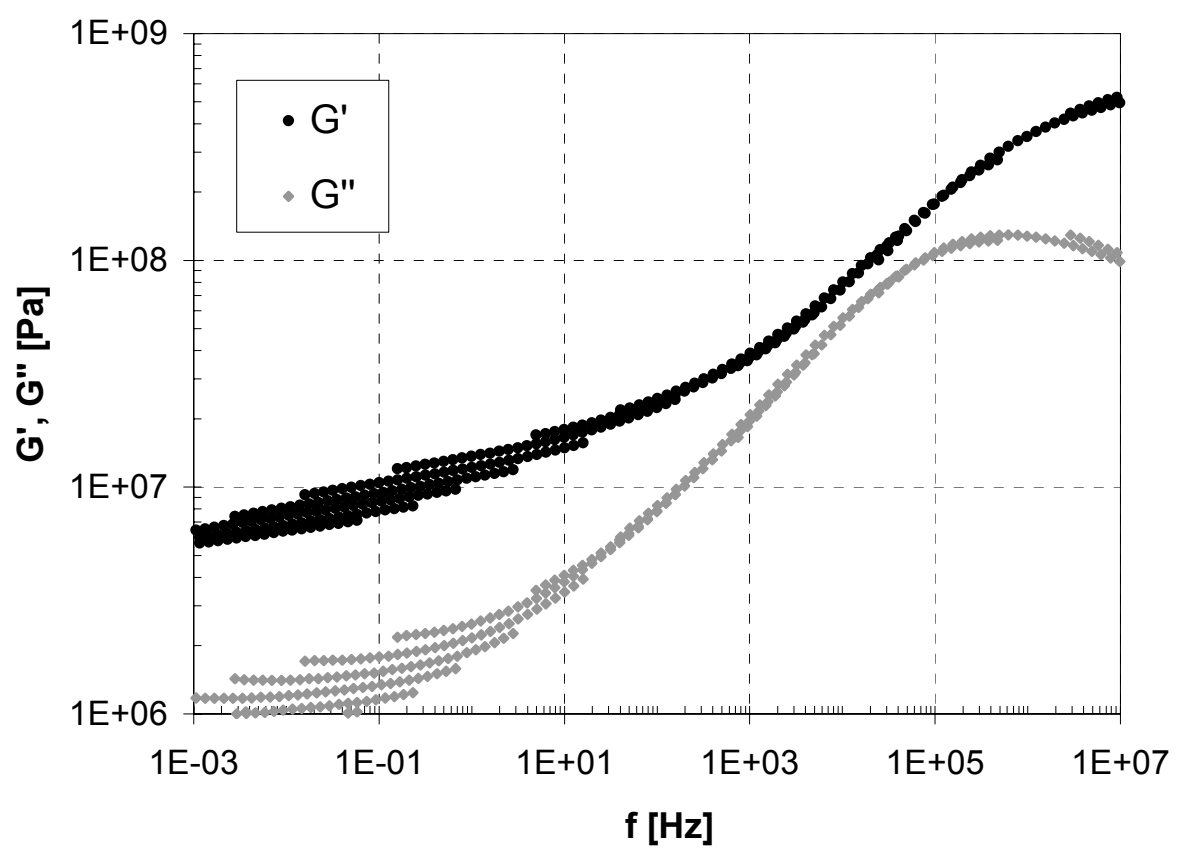

Figure 5.12: Master curve of dynamic moduli for a carbon black filled S-SBR5025 after horizontal shifting procedure according to Figure $5.11\left(\mathrm{~T}_{\text {ref }}=20^{\circ} \mathrm{C}, \varepsilon=0.5 \%\right)$

The manifestation of this effect can be also detected by analysing temperature sweeps performed at a fixed frequency, whereby a constant decrease of the elastic modulus is observed above the glass transition regime for filled compounds. By plotting the elastic modulus as a function of the inverse temperature, an Arrhenius like process can be followed and characterised by $\log \left(\Delta G^{\prime}\right) \sim E_{a} / R T$, where $E_{a}$ denotes an activation energy (Figure 5.13). 
The evaluated activation energy is physically related to the thermal activation of filler-filler bonds and will be discussed further on in this section [66].

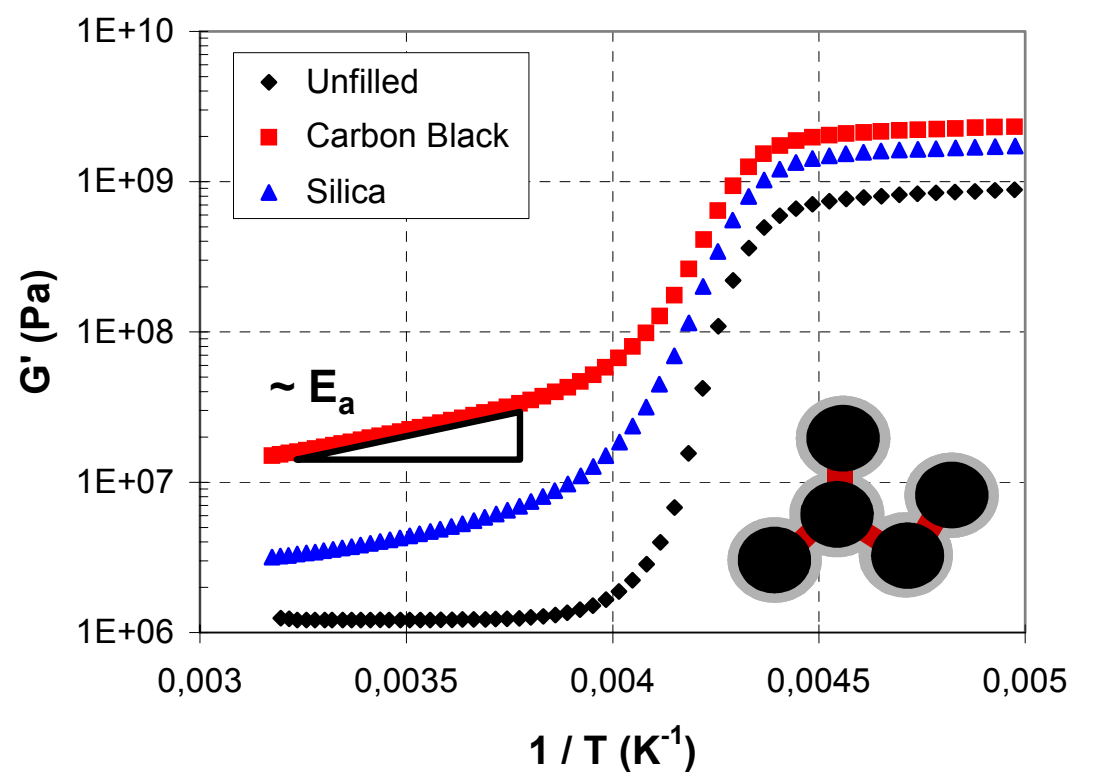

Figure 5.13: DMA Activation Plot of the storage modulus over the inverse temperature for a S-SBR 2525 basis polymer (f $=1 \mathrm{~Hz}, \varepsilon=0.5 \%$ ). Scheme: Filler particles surrounded by bound rubber and connected by glassy polymer bridges

From a morphological point of view, the insertion of a filler structure in the polymer matrix strongly modifies the polymer dynamics in the vicinity of filler particles, where the interaction with the filler surface hinders the chain mobility. For attractive interactions, the so-called "bound rubber" layer around filler particles exhibits a slowed-down dynamics, implying that the local glass transition of the polymer nanolayers close to the surface occurs at significantly higher temperatures than the one from the bulk polymer matrix. This effect can typically be observed in polymer thin films and within the confining area between flocculated filler particles, which are separated by nanoscopic bridges of immobilised polymer [54][55][77] [78][79]. At moderate temperatures the polymer layers between flocculated filler particles are considered to be in a glassy state. Since these glassy polymer bridges are well attached to the filler surface, they can transmit large stresses between adjacent particles, leading to a high stiffness and strength of filler-filler bonds. In turn, the stiffness and strength of the bonds and its thermal activation governs that of the filler network and the whole rubber sample [54][62]. The associated structure of filler networks in elastomers is depicted in Figure 5.13, where the bound rubber and the glassy polymer bridges are indicated. The mechanism related to the thermal activation of glassy polymer bridges is associated with an activation energy which can be directly evaluated via an Arrhenius plot of the temperature dependent dynamic storage modulus. The Arrhenius-like feature exhibited above the glass transition 
temperature of the bulk polymer indicates the local nature of thermally activated mechanisms related to the presence of filler.

Since dielectric results indicate that the semi-empirical WLF functions are not influenced by filler content for silica filled composites, the poor overlapping of dynamic moduli in the low frequency region can not be simply compensated by an additional horizontal shift. Instead, vertical shift factors $f_{v}$ are introduced to describe the temperature dependent softening behaviour of filled elastomers above glass transition. As shown in Figure 5.12, the decrease of modulus is not identical whether the storage or the loss modulus is considered, which suggests the occurrence of two distinct thermally activated mechanisms. Consequently, vertical shift factors are separately applied to each modulus component. An example is shown in Figure 5.14 for both components of dynamic modulus originally presented in Figure 5.12. The left part of the curve - high temperatures - corresponds to the activation regime where isothermal segments are vertically shifted with respect to the reference temperature. Thereby, an Arrhenius dependence of vertical shift factors is found and physically interpreted as thermally activated processes with a probability of occurrence equal to $\exp \left(-E_{a} / R T\right)$, where $E_{a}$ is the activation energy and $R$ is the gas constant. When approaching the glass transition, since the chain mobility is progressively reduced in the bulk material, thermally activated processes are frozen and vertical shift factors tend to zero.

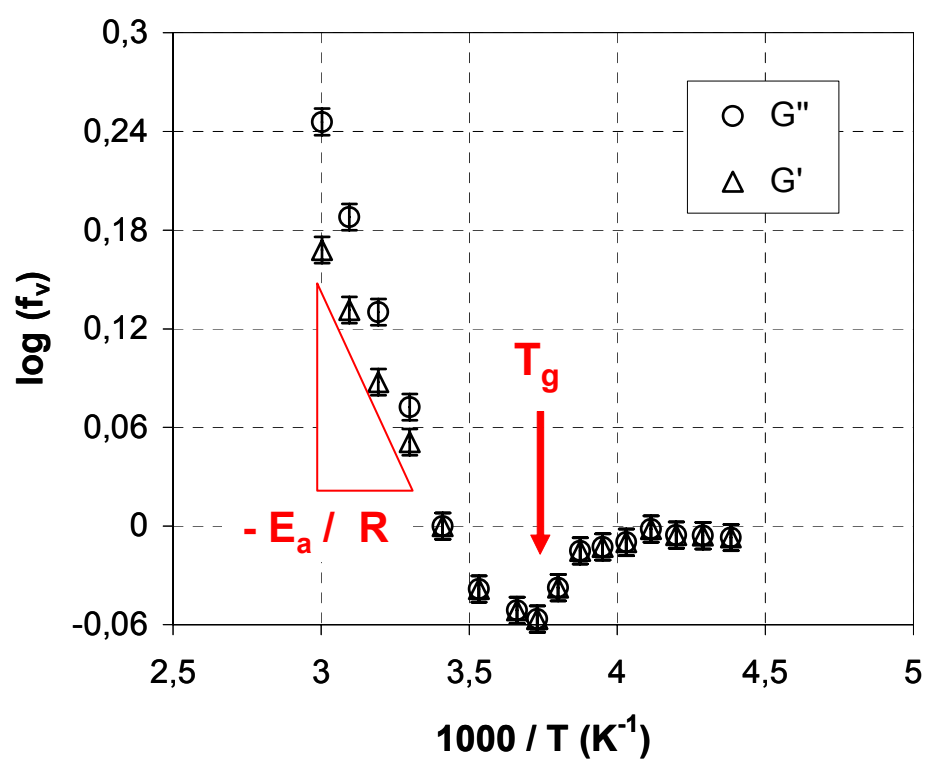

Figure 5.14: Vertical shift factors as a function of the inverse temperature for carbon black filled S-SBR 5025

The corresponding activation energy of the temperature dependent vertical shift factors is physically interpreted as the activation energy of the glassy polymer bridges in the vicinity of 
nanoscopic gaps between adjacent filler particles. By applying the same procedure to the dynamic storage modulus, a unique a master curve is constructed over a wide range of frequency. Figure 5.15 depicts the frequency dependent dynamic moduli for a carbon black filled S-SBR 5025 characterised at a strain amplitude $\varepsilon=0.5 \%$ and mastered with a reference temperature $\mathrm{T}=20^{\circ} \mathrm{C}$. In a similar way, master curves can be obtained for the other investigated composites and the corresponding results are presented in Figure 8.3 (Appendix).

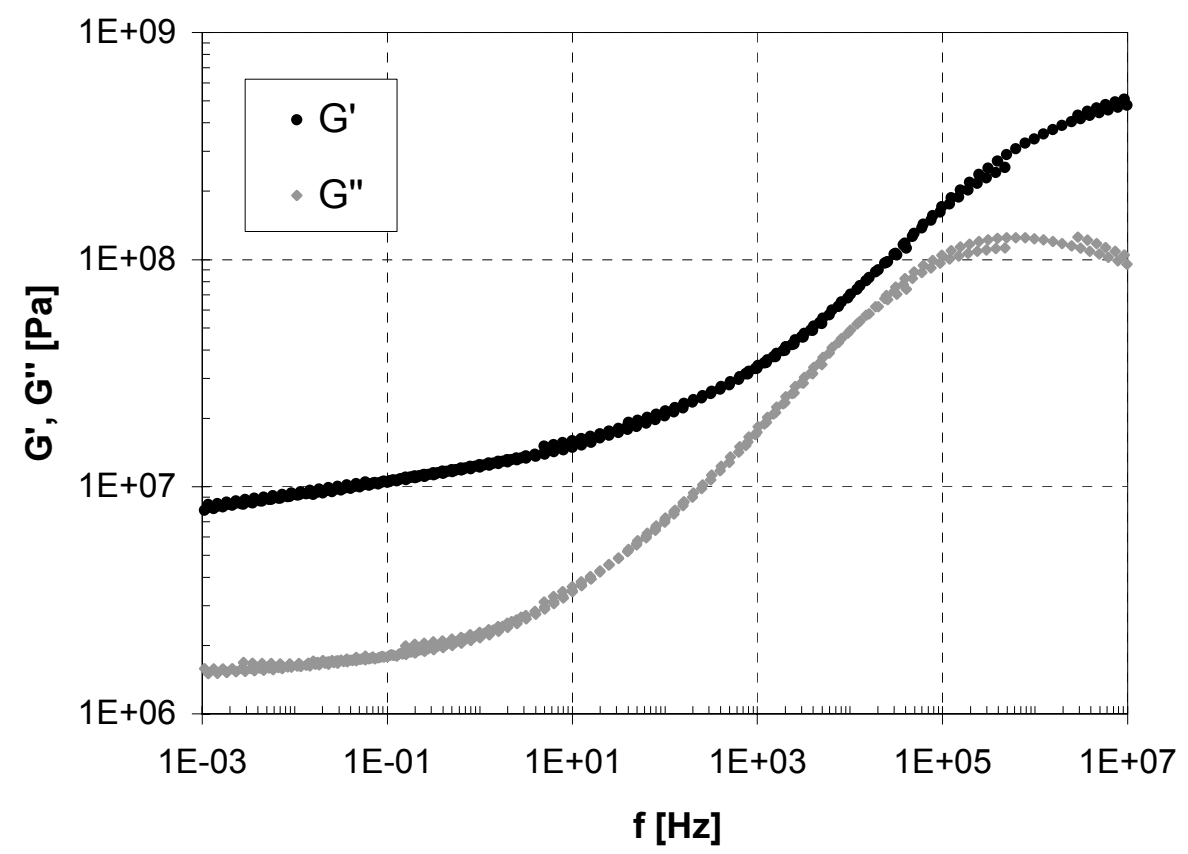

Figure 5.15: Final master curve of dynamic moduli for a carbon black filled S-SBR5025 after application of vertical shift factors $\left(\mathrm{T}_{\text {ref }}=20^{\circ} \mathrm{C}, \varepsilon=0.5 \%\right)$

The results of the vertical shifting procedure are summarised in Table 5.3. The activation energy values are depicted for temperature and frequency sweeps with a distinction between both modulus components for the latter. The values are found to be in the order of $10 \mathrm{~kJ} / \mathrm{mol}$, corresponding to the physical range of Van der Waals interactions and also corroborating previous values presented in the literature [54][79]. The activation energy $E_{a}$ associated with the G' component appears to be systematically smaller than the one related to G". By referring to the dynamic flocculation model of rubber reinforcement, the two activation processes can be traced back to the thermal activation of two kinds of filler-filler bonds, e. $\mathrm{g}$. the glassy polymer layers between filler particles, which become softer with rising temperature [55]. The activation energy associated with $G^{\prime}$ is related to an Arrhenius behaviour of the force constant of filler-filler bonds in a virgin state, while the activation energy of G" corresponds to the force constant of softer filler-filler bonds in a damaged state, 
resulting from stress induced breakdown and re-aggregation of the bonds during cyclic deformations. With this concept the temperature dependency of the characteristic stress softening effect as well as filler induced hysteresis up to large strain could be well explained, whereby the activation energies obtained from the vertical shifting procedure at $3.5 \%$ strain amplitude provided reasonable simulations for the stress-strain cycles between $5 \%$ and $100 \%$ strain [62]. These results indicate that the larger activation energies found for the very small strain amplitudes are possibly related to a dispersion effect of bond structures, e. g. a fraction of weaker filler-filler bonds which break initially at small deformations.

For comparison reasons, the activation energies obtained directly from temperature sweeps of G' are shown in Table 5.3 as well. They are found to be systematically larger than the one from the frequency sweeps, because for temperature sweeps the dynamics of the filler-filler bonds is superimposed with that of the polymer matrix. This is in contrast to the mastering technique presented in Figure 5.12, where both dynamical processes are separated via the vertical and horizontal shifting procedure.

\begin{tabular}{cccccc}
\hline & \multicolumn{2}{c}{ S-SBR 5025 } & \multicolumn{2}{c}{ S-SBR 2525 } & E-SBR 1500 \\
& N339 & Silica & N339 & Silica & N339 \\
\hline G' vert. Shift & 8.81 & 2.34 & 3.17 & 1.27 & 4.16 \\
G” vert. Shift & 15.26 & 12.22 & 7.66 & 3.94 & 4.90 \\
Temp.Sweep & 11.57 & 7.93 & 10.55 & 10.40 & 10.80 \\
\hline
\end{tabular}

Table 5.3: Activation energy values from temperature and frequency sweeps $(\varepsilon=0.5 \%)$

An analysis of the results shown in Table 5.3 makes clear that two main effects must be mentioned. On the one side, high vinyl content compounds exhibit higher activation energy values. On the other side, the samples filled with silica are associated with lower activation energy values. The concept of filler-filler bonds depicted in Figure 5.15 allows for an explanation for this trend, because the thermal activation of the glassy polymer bridges is expected to be affected by the interaction strength between polymer and filler surface. Then the difference between S-SBR grades can be explained by the higher amount of double bonds and styrene units contained in the SBR 5025, which promotes the interaction between polymer and filler particles. The silica particles are in both cases chemically coupled with a silane agent to achieve a strong bonding between the polymer chains and the filler particle. This implies that the polymer-filler coupling is constituted with single "contact points", corresponding to the spatial location of the silane cross-links. On the contrary, the interaction between carbon black and the bulk polymer is dispersive and therefore materialised by an 
adsorbed layer of polymer chains with a modified dynamics in the vicinity of the filler particle. The interface is in this case distributed over the carbon black surface, which means that a large amount of polymer chains is involved in the bonding process. As a consequence, the required energy to activate thermally the immobilised polymer layers around and between adjacent filler particles is higher for the carbon black filled composites. This leads to the conclusion that during dynamic-mechanical deformations, the silica systems are dynamically softer than the carbon black systems. It can be related to "dynamically softer" hinge-like fillerfiller bonds via the immobilised interfacial layers between neighbouring silica particles within the filler clusters or the filler network, respectively [54].

Accordingly, master curves of the loss factor are presented in Figure 5.16 for carbon black and silica filled S-SBR 5025 at a reference temperature $T=20^{\circ} \mathrm{C}$ and low strain amplitude $\varepsilon=0.5 \%$. Basic differences in reinforcement between carbon black and silica filler can be evidenced in the low and high frequency region. The silica loss peak appears to occur at a lower frequency and confirms results observed on temperature sweeps (Figure 8.4). Also, a typical crossover is found around $\mathrm{f} \sim 1 \mathrm{~Hz}$, whereby internal losses are more pronounced for the silica filled compound in the high frequency region. Therefore, by referring to the hysteresis friction model, one should expect higher friction values for this silica composite under wet conditions and at sliding velocities around $v \sim 1 \mathrm{~m} / \mathrm{s}$.

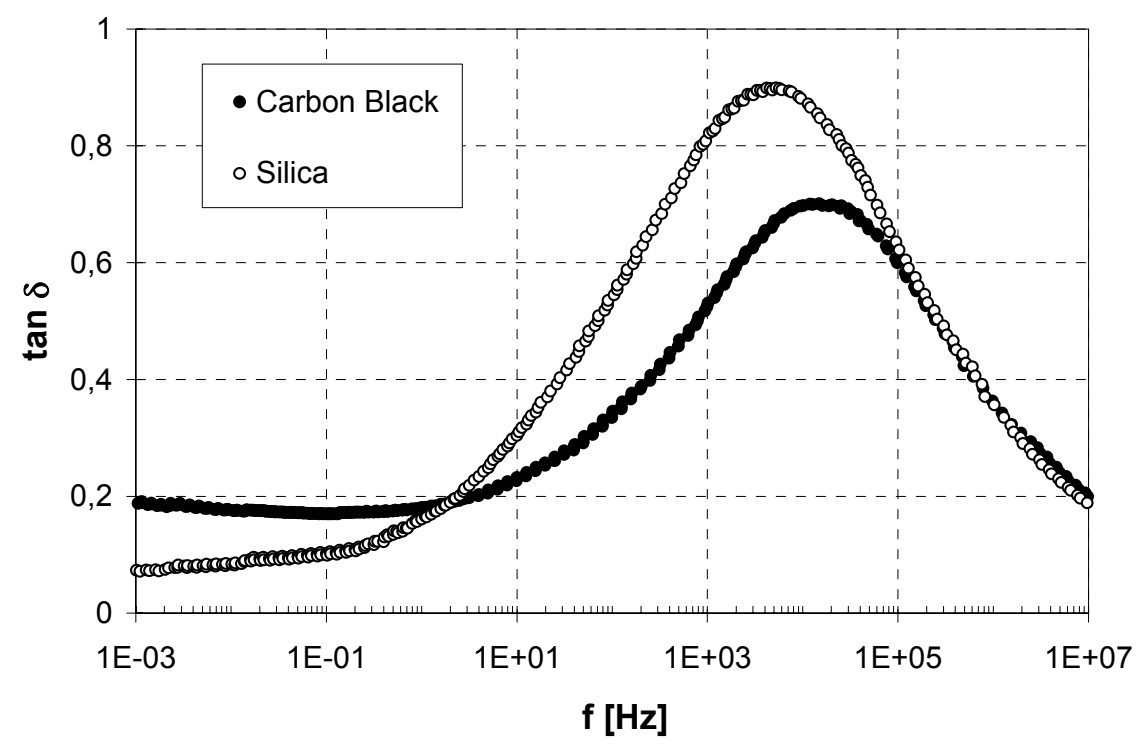

Figure 5.16: Master curve of loss factor for silica and carbon black filled S-SBR5025 $\left(\mathrm{T}_{\text {ref }}=20^{\circ} \mathrm{C}, \varepsilon=0.5 \%\right)$

The examination of the low frequency range reveals a completely opposite trend as the carbon black filled system exhibits a higher level of material losses. Based on a similar argumentation as for friction, the level of rolling resistance correlated with the loss factor at 
low frequency [57][58]. Consequently, in addition to improved friction properties, the silica filled system should be associated with a lower rolling resistance as suggested by the development of green compound for tyre treads [5]. In addition, the corresponding frequency dependent dynamic moduli are shown in Figure 8.3 (Appendix).

Frequency dependent loss factors are presented in Figure 5.17 for different SBR grades filled with the same carbon black content, namely $60 \mathrm{phr}$, whereby master curves were constructed at the same reference temperature $\mathrm{T}_{\text {ref }}=20^{\circ} \mathrm{C}$ and strain amplitude $\varepsilon=0.5 \%$. As displayed on temperature sweeps (Figure 8.4), the relative position of the $\tan \delta$ peaks is controlled by the polymer type and can be traced back to the respective transition temperatures: indeed, the lower the glass transition temperature, the higher the frequency at which the dynamic glass transition occurs. Also, material losses are found to be more pronounced for the S-SBR 5025 basis polymer above the glass transition regime. The corresponding master curves of dynamic moduli are depicted in Figure 8.3 (Appendix).

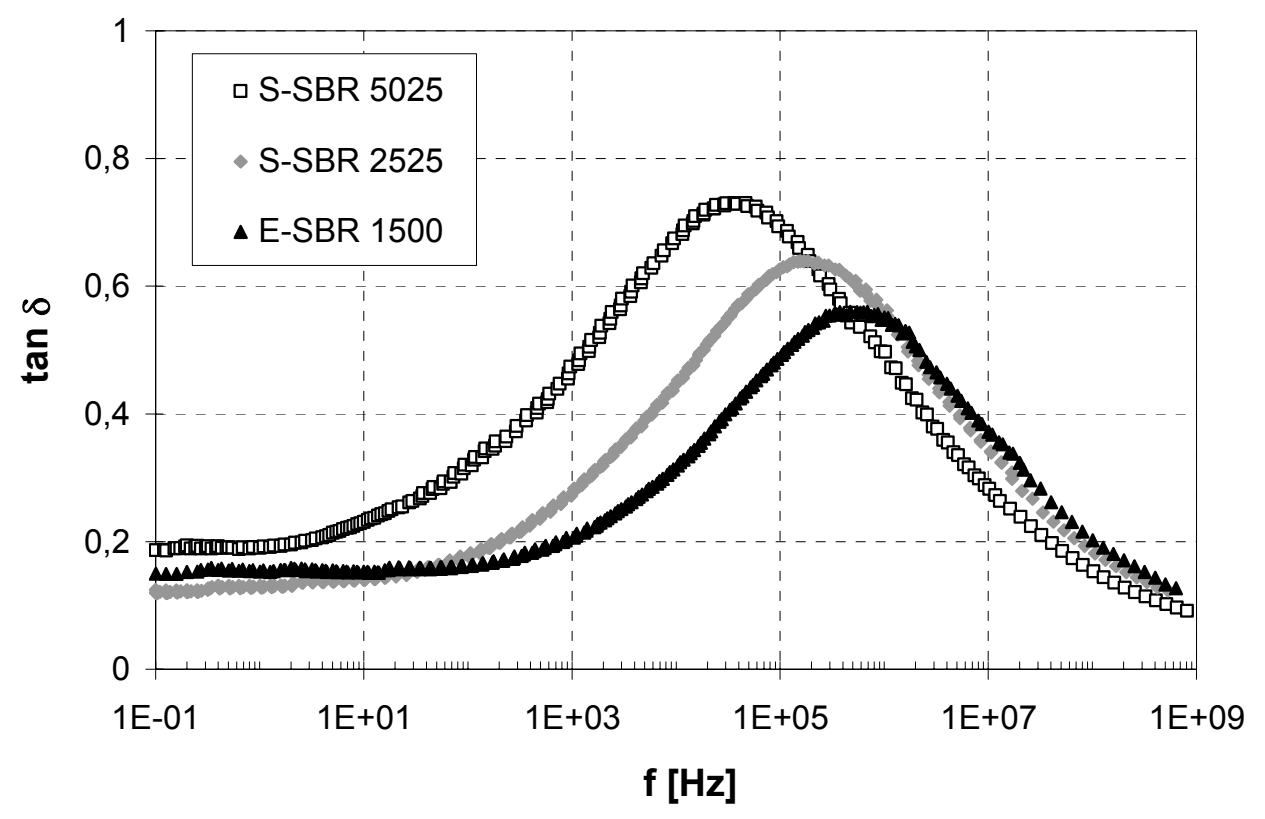

Figure 5.17: Master curve of loss factor for carbon black filled composites with different SBR grades $\left(\mathrm{T}_{\text {ref }}=20^{\circ} \mathrm{C}, \varepsilon=0.5 \%\right)$

The addition of filler significantly improves rubber mechanical properties via a reinforcement mechanism but introduces at the same time a non-linear dependency upon dynamic strain amplitude, generally denoted as Payne effect in the literature [6][7]. Thereby, the loss factor generally increases with increasing strain amplitude up to $10 \%$. Since high damping properties are directly connected to the phenomenon of heat generation, their amplitude 
should be confined to an acceptable level in order to avoid an alteration of material performance under dynamic conditions.

The occurrence of local high strains during the contact between a flat elastomer sample and a rigid spherical asperity has been highlighted in the former section by means of photogrammetry experiments. Beyond the investigation of model assumption, the results obtained raise the question of which strain amplitude should be used for the description of dynamic contact during rubber sliding friction on rough surfaces.

Experimentally, the feasibility of mechanical measurements at large deformations is principally limited by the maximum displacement and rubber specimen stiffness in the range of low temperatures. Thereby, a complete description of the glass transition process of elastomers is often hindered. This is illustrated by the master curve of a carbon black filled SSBR 5025 measured on Ares rheometer and shown in Figure 5.18. Thereby, the highest strain amplitude experimentally accessible $-\varepsilon=3.5 \%$ - implies a strong increase of the loss factor over the whole frequency range. Since the frequency dependent loss modulus directly enters in the formulation of the hysteresis friction coefficient, model predictions should be strongly affected by the strain amplitude at which dynamic measurement is performed. A recent work confirms the impact of strain amplitude on hysteresis friction simulations [73][74].

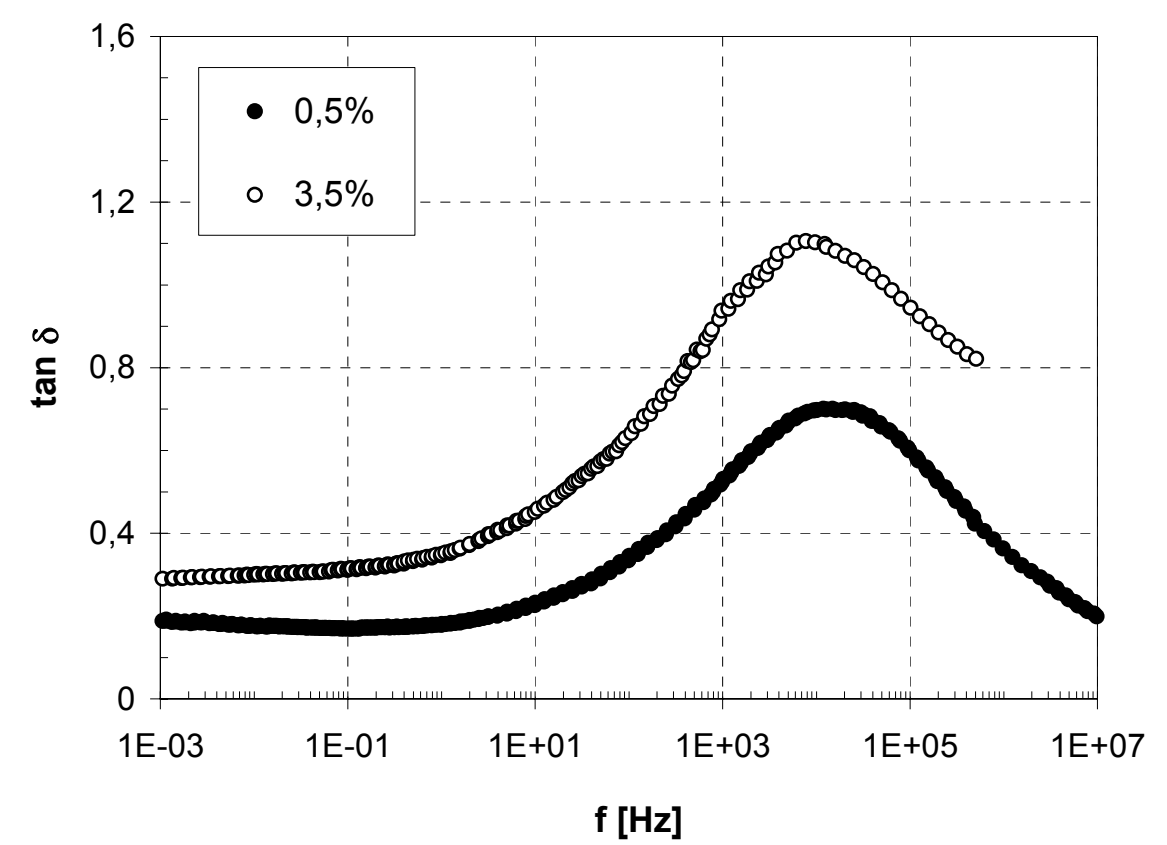

Figure 5.18: Master curve of loss factor for a carbon black filled S-SBR 5025 at two different dynamic strain amplitudes $\left(T_{\text {ref }}=20^{\circ} \mathrm{C}\right)$ 


\subsection{Simulations of hysteresis friction}

This section exclusively presents simulations performed with the hysteresis friction model in its simple and extended form (one and two scaling regimes). The influence of various factors on contact parameters is highlighted: polymer grade, filler, surface, load, sliding velocity and temperature. In particular, the load dependence is interpreted via the Greenwood-Williamson functions, underlining the close relationship between load and sliding velocity during dynamic contact. Finally, a schematic empirical temperature distribution is implemented into the hysteresis friction model through which a deeper insight on the impact of temperature on contact parameters at high sliding velocities can be gained.

\subsubsection{One scaling regime}

When simulating the hysteresis friction coefficient, the simplest situation involves an unfilled elastomer sliding on a rough surface whose roughness is characterised by a single scaling regime. As an example, the hysteresis friction coefficient $\mu_{H}$ is depicted in Figure 5.19 for two unfilled S-SBR composites as a function of the sliding velocity at laboratory temperature $\mathrm{T}=23^{\circ} \mathrm{C}$. More generally, the temperature is kept constant at $\mathrm{T}=23^{\circ} \mathrm{C}$ for both simulations and experimental results except in the sub-section "Load dependence and temperature effects". This implies a slight correction for the master curves presented in the previous section at a reference temperature $T_{\text {ref }}=20^{\circ} \mathrm{C}$. The rough granite surface characterised in Section 5.1 is used as substrate with a one-scaling-regime approach for the description of roughness. Dynamic moduli required for the simulations were characterised at low strain amplitude $\varepsilon=0.5 \%$. The impact of strain amplitude on the hysteresis friction is only considered at the end of this section. The load $\sigma_{0}=12.3 \mathrm{kPa}$ corresponds to the experimental load used for stationary friction measurements for low sliding velocities (Chapter 4). By keeping the load at a low level, the interfacial temperature increase is assumed to be negligible over the experimental range of sliding velocities. However, it should be noted that the temperature increase at high sliding velocities is first not considered in this section, so that the emphasis is solely put on viscoelastic features of hysteresis friction.

For comparison purposes, the hysteresis friction coefficient $\mu_{H}$ is normalized for both S-SBR grades (Figure 5.19). Viscoelastic features previously observed on dynamic moduli are clearly reflected by the simulation. Indeed, the position of the hysteresis friction maximum is shifted towards higher sliding velocities with decreasing vinyl content, e.g. decreasing glass transition temperature. Knowing that the frequency of excitation during sliding friction on rough surfaces is proportional to the sliding velocity (see Equation (2.18), the impact of 
polymer architecture experimentally determined by means of relaxation spectroscopy can be followed by the hysteresis friction. It is interesting to note that the distance between both friction peaks is identical to the one found for the maxima of loss modulus, which confirms previous results and illustrates the viscoelatic nature of dynamic contact between elastomers and rough surfaces [13].

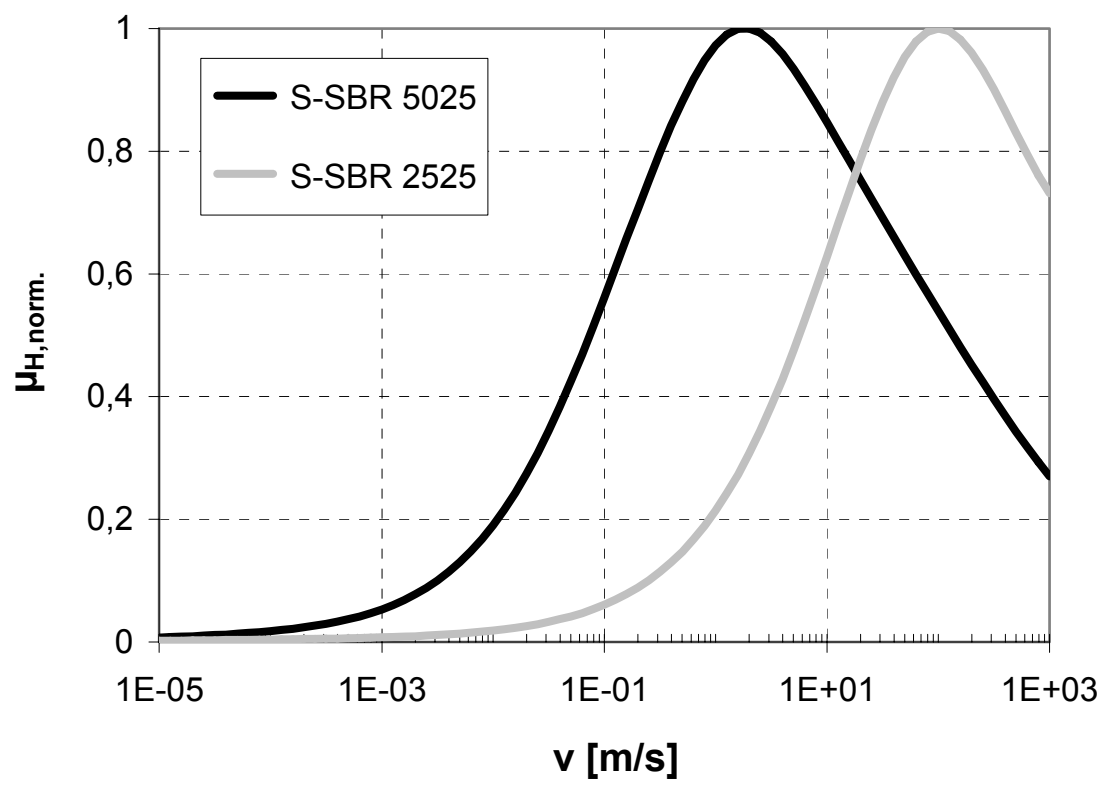

Figure 5.19: Normalized simulated hysteresis friction coefficient for unfilled S-SBR grades on rough granite. Load $\sigma_{o}=12.3 \mathrm{kPa}$

Another aspect of viscoelasticity can be investigated via the temperature dependency of hysteresis friction curves. Figure 5.20 shows the normalized hysteresis friction coefficient $\mu_{H}$ simulated for an unfilled S-SBR 5025 under the above described conditions with a variation of the temperature from $\mathrm{T}=0$ to $\mathrm{T}=60^{\circ} \mathrm{C}$. As expected, the position of the friction maximum is directly related to the temperature with a pronounced shift of the friction peak towards high sliding velocities when increasing temperature. This behaviour can be directly traced back to the semi-empirical WLF/VFT functions which govern the temperature and frequency dependency of viscoelastic quantities: the same effect would be obtained by considering a higher reference temperature while mastering the dynamic moduli. Beside the shift of the friction peak, the simulation exhibits identical features with a characteristic increase below as well as decrease above - the friction maximum. This is due to the master procedure applied for unfilled compounds, whereby dynamic moduli curves are solely shifted horizontally. 


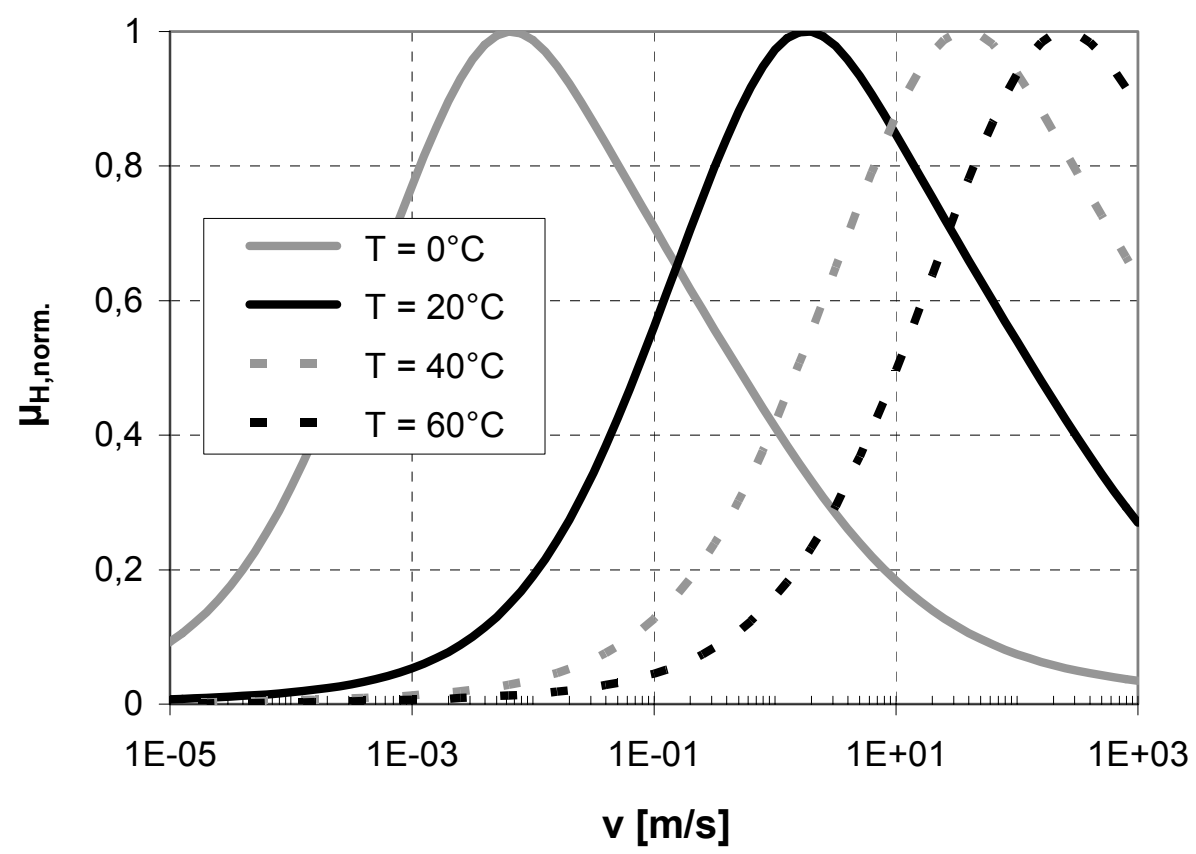

Figure 5.20: Normalized simulated hysteresis friction coefficient for unfilled S-SBR 5025 on rough granite at various temperatures. Load $\sigma_{o}=12.3 \mathrm{kPa}$

The addition of filler leads to a significant change of dynamic properties: depending on the filler type and content, defined reinforcing effects can be achieved within a broad range of frequency. For instance, while the carbon black filled S-SBR 5025 exhibits a high level of internal losses in the low frequency region, the incorporation of silica with coupling agent results in a more pronounced loss peak in the dynamic glass transition region (Figure 5.16). The impact of filler on the simulated hysteresis friction coefficient $\mu_{H}$ is shown in Figure 5.21 for a S-SBR 5025 basis polymer. As indicated by the logarithmic vertical scale, the level of generated hysteresis friction for the unfilled composite at very low sliding velocities is dramatically low and further on shows a pronounced increase of more than two decades up to a maximum located at $\mathrm{v} \sim 3 \mathrm{~m} / \mathrm{s}$, as suggested by the frequency dependent loss factor. The simulated hysteresis friction of filled composites reflects features observed on the loss factor depicted in Figure 5.16, namely a typical crossover of silica and carbon black filled SSBR 5025 at low sliding velocities. However, the path of $\mu_{H}$ curves can not be completely understood by solely referring to the frequency dependent loss factor $\tan \delta$. The hysteretic response of elastomers during the sliding process arises from the roughness spectrum of excitation of the substrate, e.g. the ability of rubber to form intimate contact with asperities over several length scales. 


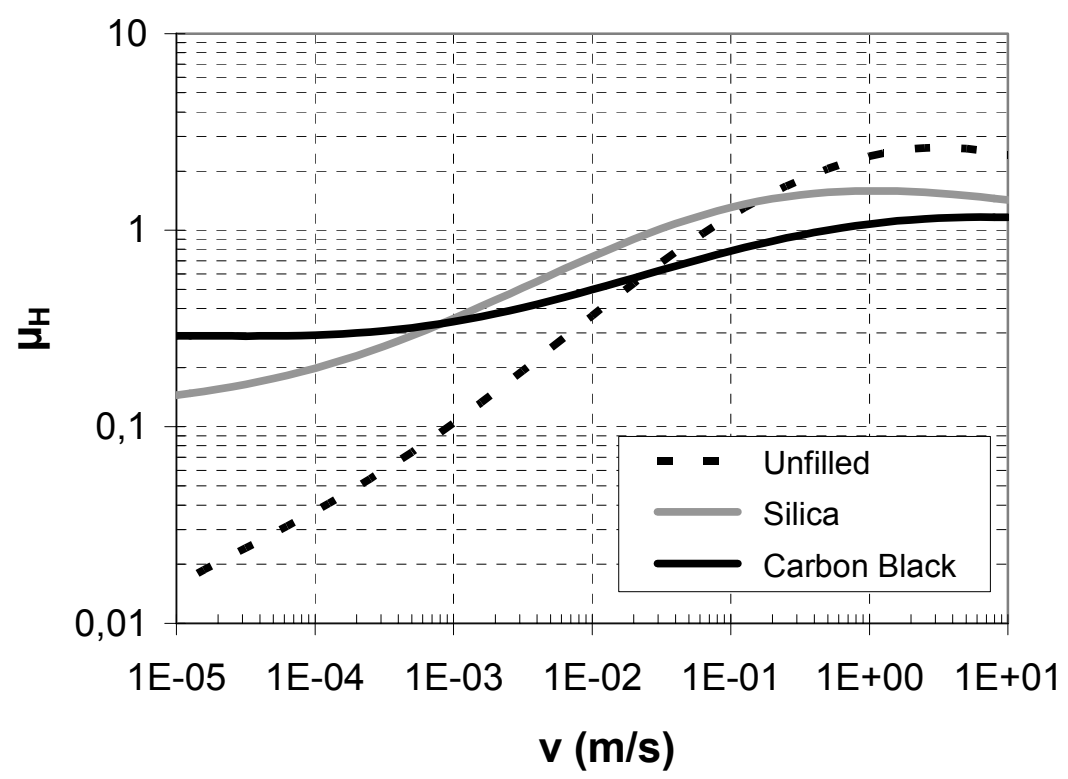

Figure 5.21: Simulated hysteresis friction coefficient for filled and unfilled S-SBR5025 on rough granite. Load $\sigma_{o}=12.3 \mathrm{kPa}$

Up to here, the rough substrate is viewed as a multi-scale source of excitation which dynamically acts on the elastomer sliding over it. Accordingly, the viscoelastic response of rubber leads to the generation of tangential stresses contributing to the friction force. Since the friction integral includes both surface and material quantities, the role played by different length scales can be hardly identified. Then, in order to gain a quantitative estimation of the contribution of roughness during sliding process, the simulated hysteresis friction coefficient was decomposed into three friction integrals associated with distinct contact intervals: a first range $\lambda=\left[100 \mu \mathrm{m}-\xi_{\| 1}\right]$ which encompasses the largest asperities of the profile, an intermediate one comprised between $\lambda=[10-100 \mu \mathrm{m}]$ and a last interval within the micrometer range $\lambda=[1-10 \mu \mathrm{m}]$.

Results are shown in Figure 5.22 with total and detailed contributions on the simulated hysteresis friction coefficient. It can be seen that the hysteresis friction mainly arises from the first range, i.e. the largest asperities of the profile, which contribute at approximately $80 \%$ of the total level. The contribution of the intermediate and smallest intervals is found to lie around one and two decades below the total generated hysteresis, respectively. Consequently, the macrotexture almost solely determines the level of hysteresis friction and the corresponding length scale regime requires therefore a precise description regarding surface descriptors. By referring to the roughness analysis carried out on the rough granite surface in Figure 5.2, it becomes clear that a one-scaling-range approach is not satisfying to describe the morphology of length scales larger than $\lambda=200 \mu \mathrm{m}$, which in return may 
completely mislead the prediction of hysteresis friction. In that case, the consideration of a second scaling regime at larger length scales is crucial for the relevance of simulations.

It is interesting to note in Figure 5.22 that the individual hysteresis friction maxima are shifted towards lower sliding velocities for smaller length scales. Indeed, since the excitation frequency is inversely proportional to the length scale, a material in contact with microasperities will reach the dynamic glass transition region - illustrated by the $\mu_{H}$-peak - at a lower sliding velocity compared to the one deformed by large asperities. Even if the contribution of microscopic length scales on hysteresis friction is almost negligible, the multiscale nature of contact results in a broadening of the $\mu_{\mathrm{H}}$-peak.

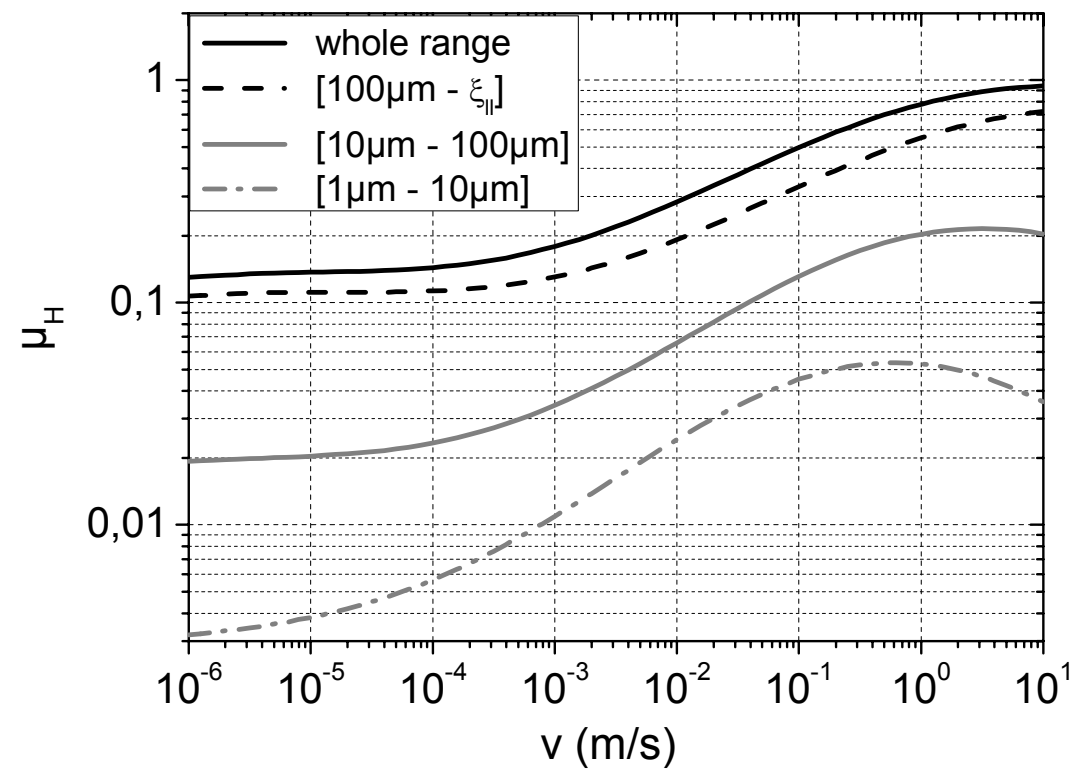

Figure 5.22: Length scale dependent decomposition of the hysteresis friction coefficient on rough granite for a carbon black filled S-SBR 5025. Load $\sigma_{\circ}=12.3 \mathrm{kPa}$

The examination of simulated contact parameters allows a deeper understanding of the mechanisms involved during rubber sliding friction on rough surfaces. In particular, the real area of contact should be more closely analysed with respect to dry friction and adhesion processes. As presented in Chapter 3, the real area of contact basically depends on the length scale at which the contact is observed. While a magnification of roughness leads to a significant reduction of the size of contact patches, their number is thereby found to dramatically increase. The analytical expression of contact area derived in Equation (3.36) shows that this quantity is governed by characteristics of both contact partners - surface descriptors and material dynamic properties. In particular, a rough surface associated with a fractal dimension $D=2.5$ displays the high amount of contact patches since fluctuations of profile height are directly related to the summit density. 
Simulations of the real area of contact are presented in Figure 5.23 for filled and unfilled S-SBR 5025 on rough granite at moderate load $\sigma_{o}=12.3 \mathrm{kPa}$. Since the interval of excitation frequency is shifted towards the dynamic glass transition region with increasing sliding velocity, the area of contact is found to exhibit a continuous decrease over the range of simulation. Indeed, due to an increase of the dynamic stiffness, the rubber is not able to fill the smallest cavities of the profile for sufficiently high sliding velocities. As expected, the unfilled - and a fortiori softer - S-SBR 5025 exhibits a higher contact area compared to filled composites. The analysis of $A_{d} / A_{0}$ values between the carbon black and silica filled S-SBR 5025 gives an indication about the filler efficiency regarding inter-locking properties. The silica filled system exhibits a significantly higher contact area compared to the carbon black filled system (about $50 \%$ more at $v=10^{-5} \mathrm{~m} / \mathrm{s}$ ), while the difference tends to become smaller at high sliding velocities $(20 \%$ at $v=1 \mathrm{~m} / \mathrm{s})$. This prediction is in accordance with the activation energy values determined during dynamic mechanical analysis, namely less pronounced thermally activated processes for silica filled compounds. This indicates that silica fillers inserted with silane as coupling agent in the polymer matrix result in dynamically softer rubber composites, as compared to carbon black.

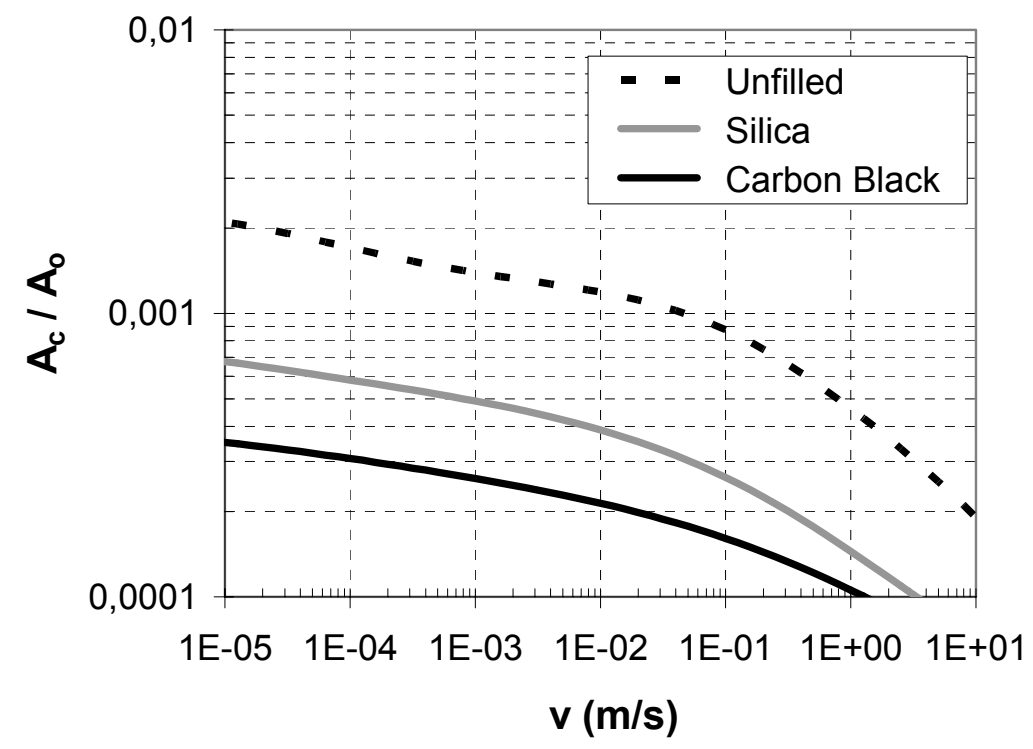

Figure 5.23: Simulated ratio between real and nominal contact area on rough granite for filled and unfilled S-SBR 5025. Load $\sigma_{0}=12.3 \mathrm{kPa}$.

Beside characteristic effects associated with material microstructure or experimental conditions, it is important, at least for filled systems, to consider the effect of strain amplitude imposed during mechanical spectroscopy on the simulated hysteresis friction. Since the presented modelling is derived according to a linear mathematical formalism, dynamic 
contact can be expressed as the sum of elementary excitation processes continuously distributed over a range of spatial frequencies. In order to take into account the complex nature of dynamic indentation processes occurring during sliding friction, the input strain at which the frequency dependent response of elastomers can be set at higher values. In this way, averaged material non-linearity can be included in a basically linear formulation. Recent friction investigations of tyre compounds on road pavement showed that the strain amplitude considerably affects the hysteresis friction feature [73][74].

Accordingly, simulations of hysteresis friction were carried out for a carbon black filled S-SBR 5025 at two different dynamic strain amplitudes $\varepsilon=0.5 \%$ and $\varepsilon=3.0 \%$ (Figure 5.24). For comparison purposes, the pre-factor of friction integral was kept constant at $\langle\delta\rangle /\left\langle z_{p}\right\rangle=1$. It can be seen that the dynamic strain directly scales up the level of hysteresis friction over the whole range of sliding velocity, as previously observed on master curves of the loss factor $\tan \delta$ (Figure 5.18). Additionally, the consideration of high dynamic strain amplitudes appears to flatten the shape of hysteresis friction and leads to a broader bell-shaped curve. Recently, friction results of filled elastomers on a silicon carbide surface obtained under wet conditions - with detergent - could be fairly described with hysteresis friction simulations performed at a strain amplitude $\varepsilon=3.5 \%$ [70]. This highlights the relevance of such an approach in case of a one-scaling-regime description of the surface morphology.

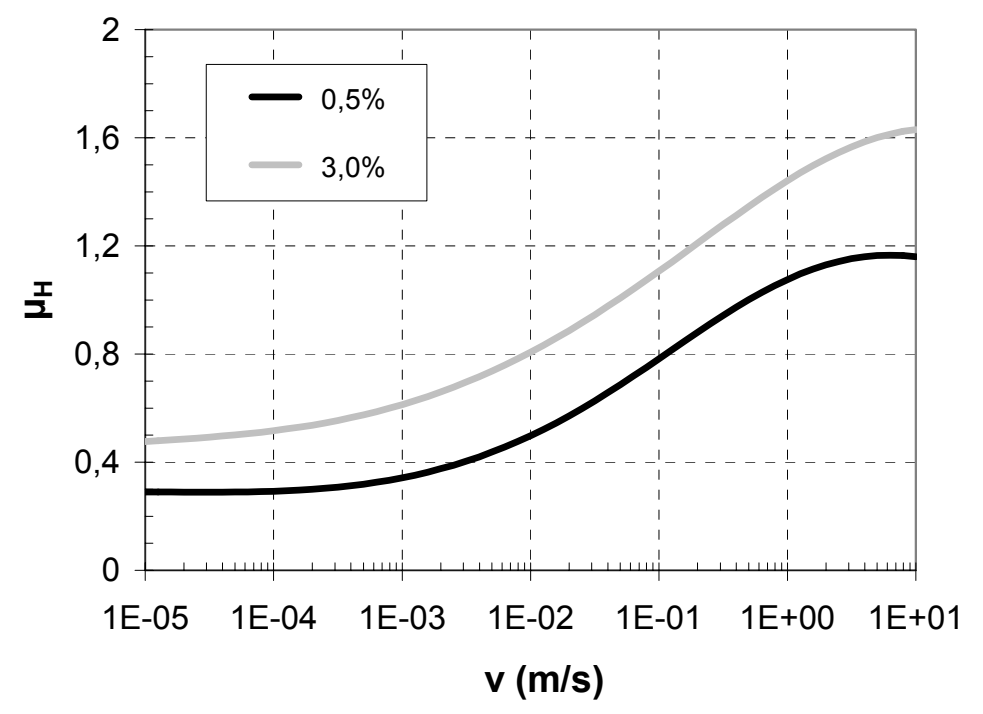

Figure 5.24: Influence of the dynamic strain amplitude $\varepsilon$ on the simulated hysteresis friction coefficient $\mu_{H}$ for a carbon black filled S-SBR 5025 on rough granite. Load $\sigma_{o}=12.3 \mathrm{kPa}$ 


\subsubsection{Two scaling regimes}

The introduction of a second scaling range enables a finer description of the height difference correlation function for large length scales which morphologically correspond to the surface macrotexture. Since surface descriptors are thereby modified, contact parameters are expected to be strongly influenced by the extended formulation of the hysteresis friction coefficient. For instance, Equation (3.42) shows that the contact interval is directly connected to the fractal dimension by a power law relationship. In the following, the impact of a two-scaling-regimes approach on the simulated hysteresis friction and contact parameters is systematically investigated. As before, the temperature is fixed at $T=23^{\circ} \mathrm{C}$ and the load accounts for $\sigma_{0}=12.3 \mathrm{kPa}$.

A plot of the hysteresis friction coefficient is shown in Figure 5.25 for silica and carbon black filled S-SBR 5025 on rough granite, whereby both simulations are adjusted to one another at very low sliding velocities for comparison purposes. By including a second scaling range, the amplitude of the $\mu_{H}$-maximum is significantly reduced of about $40 \%$ while its position is shifted towards lower sliding velocities. The typical crossover between silica and carbon black filled S-SBR 5025 is confirmed with the extended modelling and located at $v \sim 3 \mathrm{~cm} / \mathrm{s}$. Therefore, even if the enhanced description of surface roughness appears to affect the level of hysteresis friction, material features can nevertheless be evidenced.
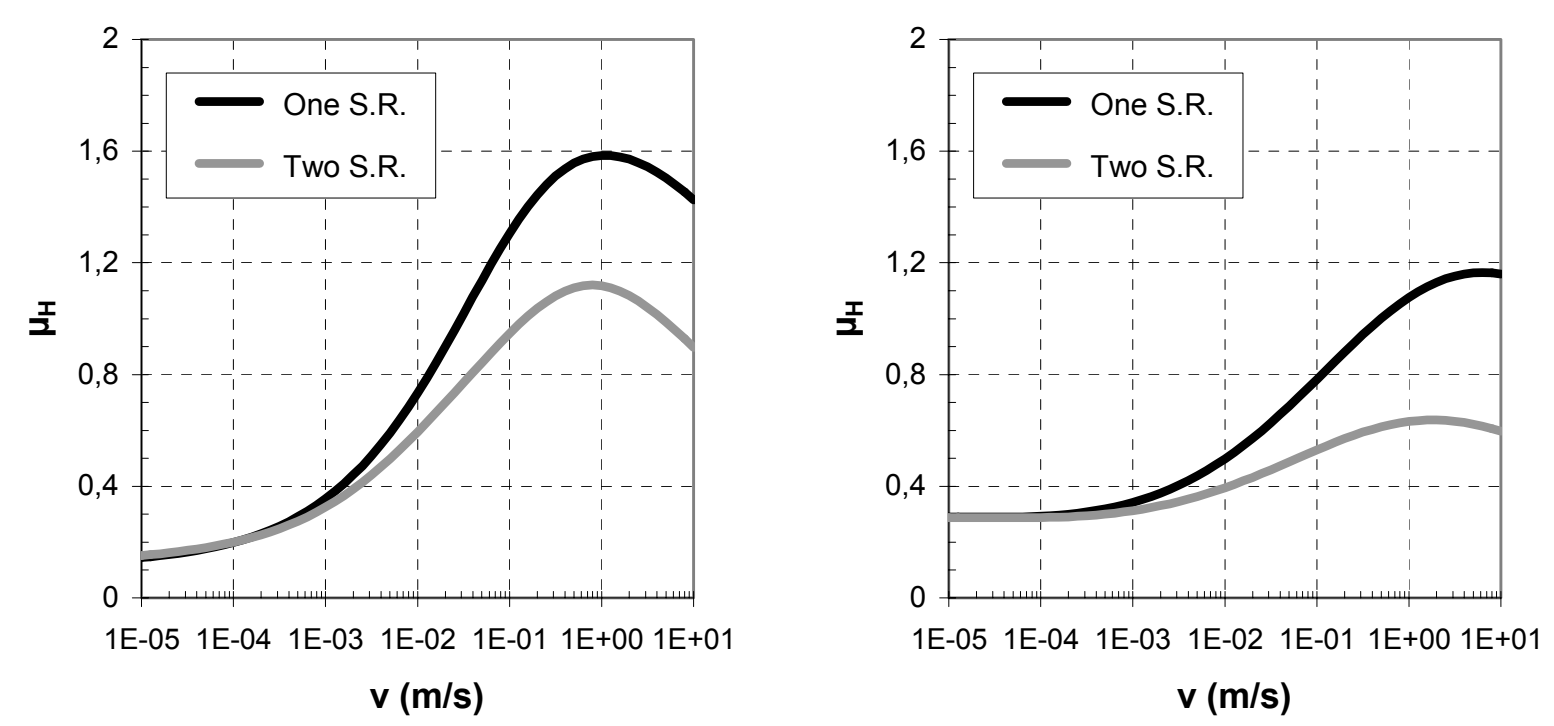

Figure 5.25: Simulated hysteresis friction for silica (left) and carbon black (right) filled S-SBR5025 on rough granite with a one and two-scaling-regimes approach. Load $\sigma_{o}=12.3 \mathrm{kPa}$ 
As presented in Equation (3.37), the two-scaling-regimes approach allows a separate determination of both micro- and macrotextures contributions at various sliding speeds and loads. A simulation of the unscaled $\left(<\delta>/<z_{p}>=1\right)$ hysteresis friction coefficient $\mu_{H}$ is shown in Figure 5.26 for a carbon black filled S-SBR 5025 on rough granite with the decomposition into micro- and macrotexture according to Equation (3.37). The macrotexture is found to mainly contribute to the hysteresis friction, confirming predictions of Figure 5.22 . This can be demonstrated by referring to the power spectrum density $S(\omega)$. Indeed, the level of the friction integral is mainly controlled by the rate at which the power spectrum density decreases. Due to the high fractal dimension of large length scales (macro-roughness), e.g. low $\beta$-exponent, the decrease of the power spectrum density with increasing sliding velocity is compensated by the dynamic loss modulus. This condition is not fulfilled for lowly fractal regimes - microroughness - so that a two-scaling-ranges modelling basically generates more hysteresis friction than a single scaling behaviour as long as $D_{1}>D_{2}$.
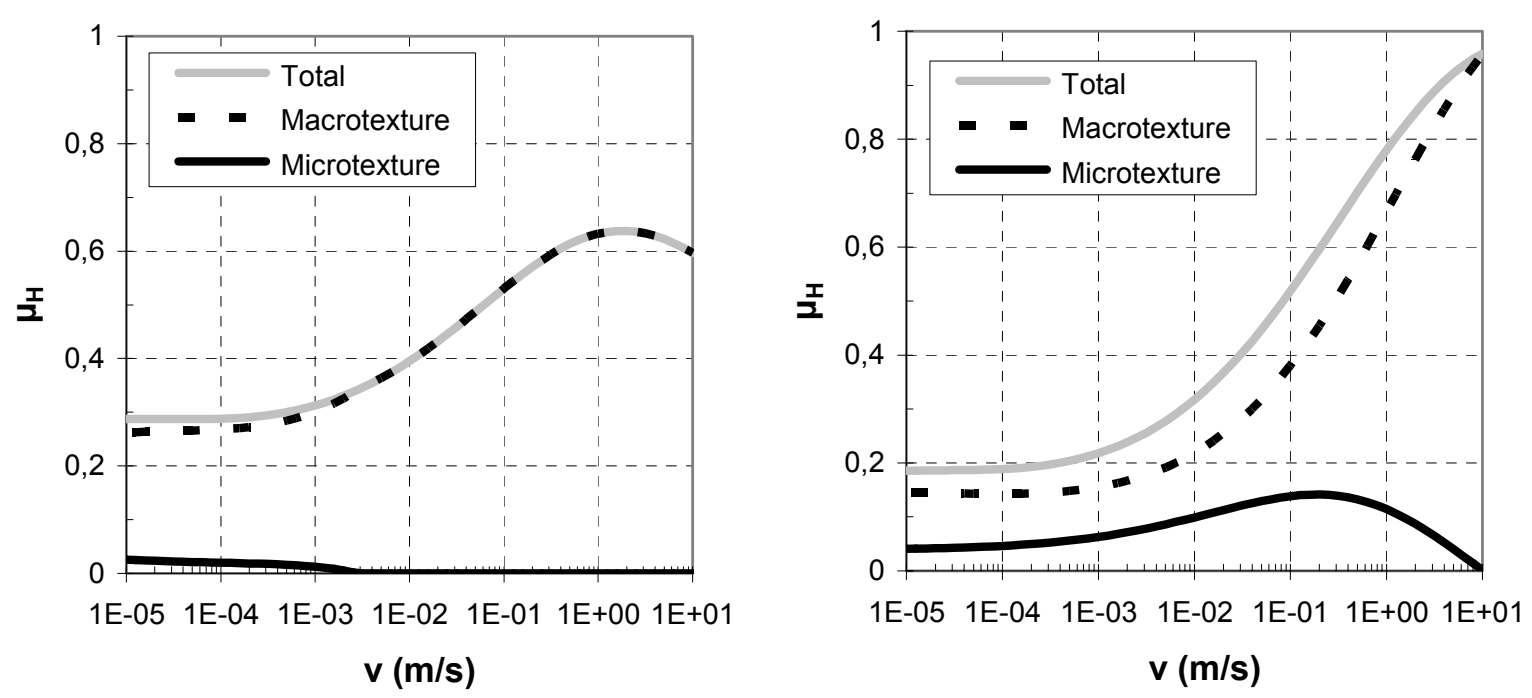

Figure 5.26: Contributions of micro- and macrotexture on the simulated hysteresis friction for a carbon black filled S-SBR 5025 on rough granite at a load $\sigma_{o}=12.3 \mathrm{kPa}$ (left) and $\sigma_{o}=250 \mathrm{kPa}$ (right)

At a moderate load $\sigma_{0}=12.3 \mathrm{kPa}$, the microtexture slightly contributes to the simulated $\mu_{\mathrm{H}^{-}}$ values up to $v \sim 3 \mathrm{~mm} / \mathrm{s}$ (Figure 5.26 left diagram). This suggests the existence of a velocity range where the minimal length scale $\lambda_{\min }$ involved in the sliding process is located above the cross-over length $\lambda_{2}$. Consequently, the impact of microtexture on the hysteresis friction coefficient appears to be gradual and only relevant whether the load is high enough or the sliding velocity is sufficiently low. Indeed, with increasing load, smaller cavities can be explored by the elastomer which in return widens the contact interval and increases the contribution of microtexture. The right diagram of Figure 5.26 clearly shows that the level of 
hysteresis friction induced by microasperities is strongly increased at high loads. Furthermore,

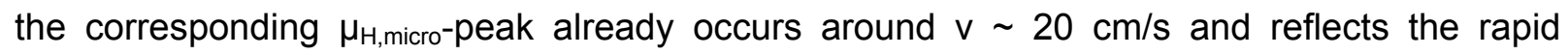
entrance of rubber into the dynamic glass transition regime due to the mechanical excitation associated with small length scales. This remark confirms experimental observations made on standard friction apparatus used by road constructors. Indeed, an experimental technique like the skid resistance tester pendulum (SRT) measuring at a sliding velocity $v \sim 3 \mathrm{~m} / \mathrm{s}$ is known to be sensitive to both macro- and microtextures, while online measurements performed at high sliding velocities - for instance with the ADHERA apparatus developed and built by the CECP in Rouen which allows the characterisation of the longitudinal friction coefficient on a blocked wheel under wet conditions up to $v \sim 30 \mathrm{~m} / \mathrm{s}$ - are solely influenced by macrotexture. Accordingly, the increase of material stiffness at high sliding speeds prevents the rubber from filling microcavities of rough substrates. This is predicted by Figure 5.26 since the contribution of the microtexture on hysteresis friction appears to be promoted with increasing load.

The examination of contact parameters shows that the modelling of roughness with two scaling ranges significantly changes the picture obtained with a single scaling regime. According to Equation (3.42), the contact interval is mainly sensitive to the exponent $\alpha=1 /\left(3 D_{2}-6\right)$. By considering a second scaling range for rough granite, one obtains $\alpha \sim 0.9$ compared to $\alpha \sim 2.4$ originally. Subsequently, the smallest length scale $\lambda_{\text {min }}$ contributing to hysteresis friction is shifted by about a decade with the two-scaling-regimes approach, thus resulting in smaller contact interval (Figure 5.27). Basic features of silica and carbon black filled S-SBR 5025 are conserved through the extended modelling as the silica filled composite exhibits lower $\lambda_{\text {min }}$ values over the whole range of simulation, e.g. a favourable inter-locking behaviour with the rough substrate. Finally, the predicted intersection between $\lambda_{\min }$ and the boundary length scale $\lambda_{2}$ makes clear that the impact of microtexture during sliding friction is expected to be significant under certain contact conditions, namely for low sliding velocities and high loads.

The reduction of the contact interval with a two-scaling-regimes approach has a direct consequence on the frequency range involved in the sliding process. Although the horizontal cut-off length $\xi_{\|}$is increased through the extended modelling, the pronounced shift of $\lambda_{\text {min }}$ leads to a diminution of the frequency interval by about a decade as shown in Figure 5.27. Consequently, the flattened shape and slight shift of the $\mu_{H}$ maximum observed in Figure 5.25 appear to be due to the shift and reduction of the frequency interval: while the former delays the increase of hysteresis friction, the latter confines the friction peak at a lower level. 

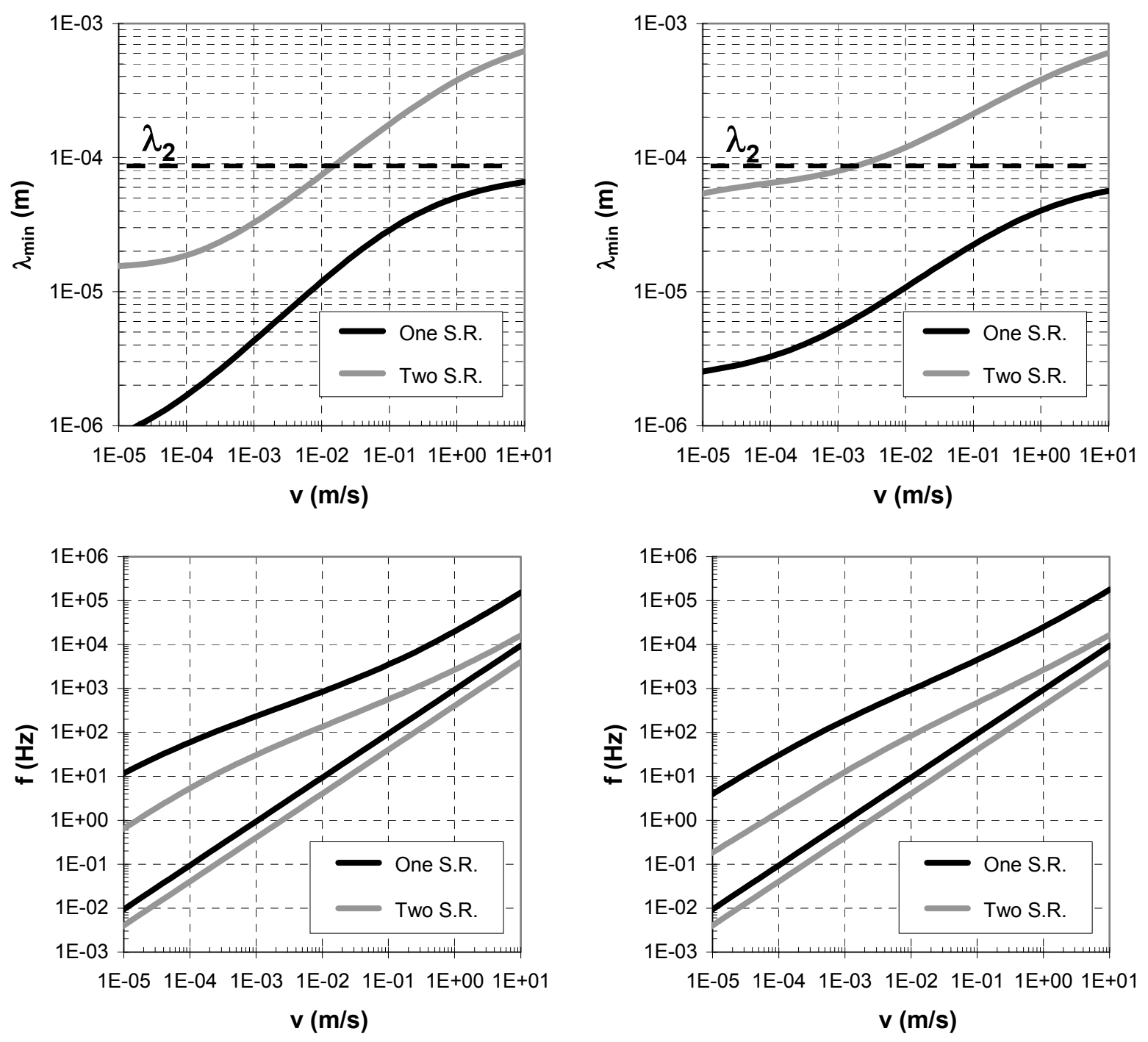

Figure 5.27: $\quad$ Simulated minimal length scale $\lambda_{\min }$ (top) and frequency interval contributing to hysteresis friction (bottom) for silica (left) and carbon black (right) filled S-SBR5025 on rough granite. Load $\sigma_{o}=12.3 \mathrm{kPa}$

The simulated real area of contact is shown in Figure 5.28 for silica and carbon black filled SSBR 5025 on rough granite with a one and two-scaling-ranges approach. Material effects related to the enhanced ability of silica compounds to form larger contact area compared to carbon black filled composites are thereby confirmed and the two-scaling-regime approach basically shifts the level of $A_{c}$ towards higher values. This can be understood by referring to Equations (3.42) and (3.43) where the shift of $\xi_{\|}$mainly explain the higher $A_{c}$ values obtained via simulations. 

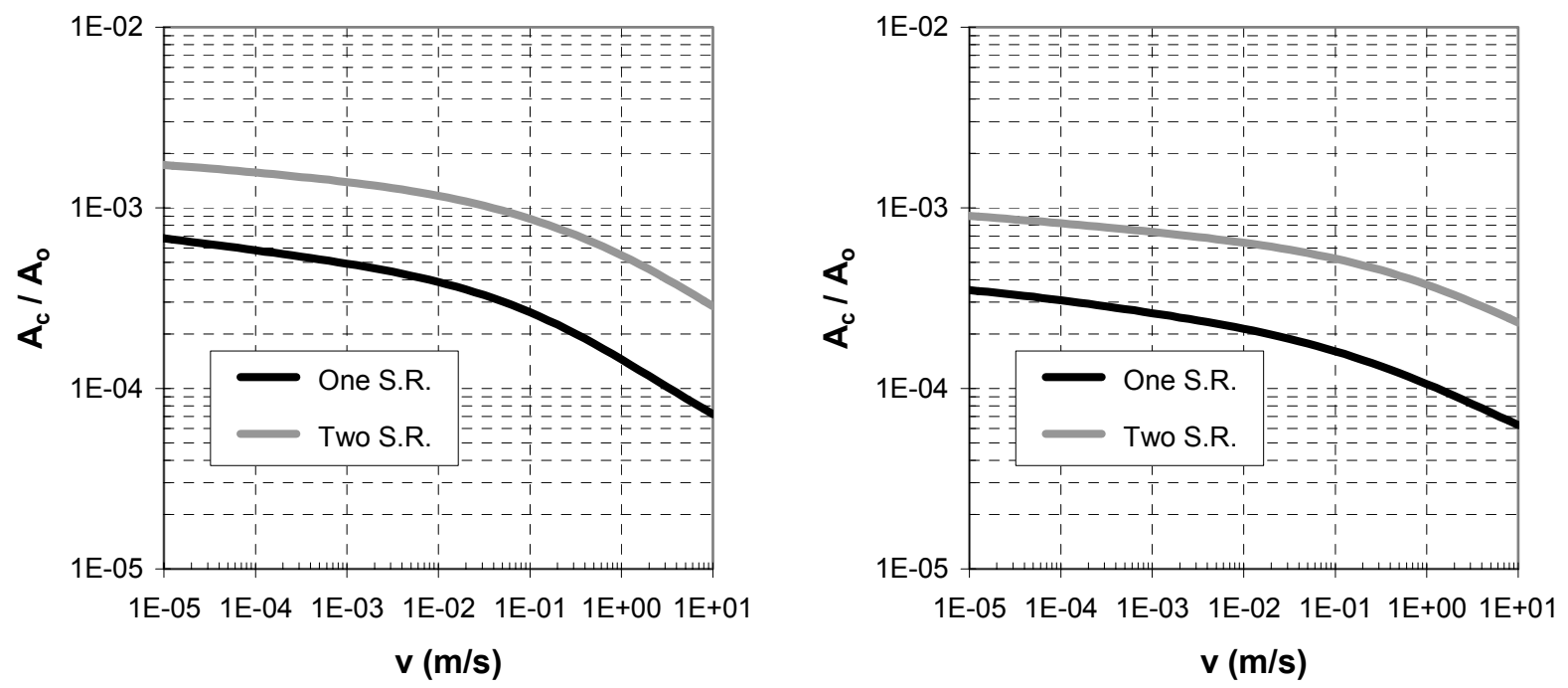

Figure 5.28: Simulated real area of contact for silica (left) and carbon black (right) filled S-SBR5025 on rough granite with a one and two-scaling-regimes approach. Load $\sigma_{o}=12.3 \mathrm{kPa}$

Finally, results concerning texture regimes can be summarized in a specific representation. The microtexture was seen to have a limited influence on the hysteresis friction coefficient. Indeed, for a sufficient high sliding velocity or low load, the rubber is not able to explore microcavities of the profile. As a result, one can identify a limit sliding velocity $v_{l}$ above which the macrotexture solely contributes to hysteresis friction (Figure 5.29). By extending the simulations to a wide range of loads, one can follow the position of $v_{1}$ for a given frictional pairing elastomer / rough surface. Figure 5.29 displays the position of a texture boundary for silica and carbon black filled S-SBR 5025 on rough granite. Below each boundary curve, the material solely "sees" the largest length scales of the profile while for the upper region of the graph micro- and macrotexture are involved in the sliding process. The comparison between silica and carbon black filled composites confirms the existence of two distinct regimes. However, the curve gives no information on the level of contact parameters like $\lambda_{\min }$ or $A_{c}$, but rather an indication on the existence of texture regimes under various contact conditions. For moderate loads up to $\sigma_{0}=30 \mathrm{kPa}$, the silica filled S-SBR 5025 shows - as previously seen in Figure 5.27 - a superior behaviour regarding the contact interval within a larger range of sliding velocity. When going over the range of high loads, the trend is inversed and the contribution of microtexture is favourable for the carbon black filled composite up to higher sliding velocities than the silica filled compound.

As presented in Chapter 2, the sole macrotexture controls the rate at which the friction coefficient decreases under wet conditions. Consequently, Figure 5.29 might be used as 
predictor and contact feature for specific frictional pairings. Knowing physical effects associated with both texture regimes and their impact on wet grip properties of tyres, the proposed representation yields as indicator for material developers to predict whether a compound is likely to fulfil experimental constraints regarding frictional behaviour under real conditions. One the other side, road constructors can use this tool in order to rate new designed pavements with the objective of optimising contact conditions for a given tyre tread compound.

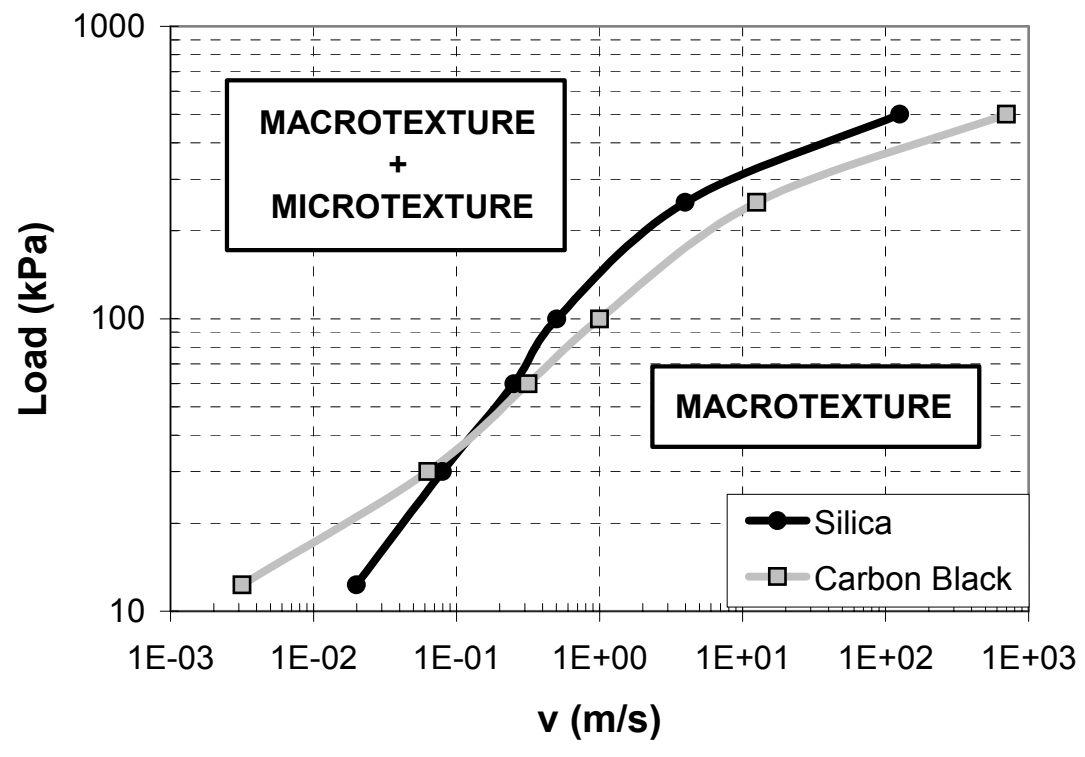

Figure 5.29: Boundary diagram with associated texture domains for silica and carbon black filled S-SBR5025 on rough granite.

\subsubsection{Load dependence and temperature effects}

Up to here, the hysteresis friction was investigated within the range of low loads and without considering the increase of contact temperature at high sliding velocities. Load and sliding velocity are intimately related to one another: the frictional power arising from dynamic contact is namely proportional to the load. Also, an analytical treatment of contact problems shows that the increase of the contact temperature is expected to vary linearly with the load [84]. Therefore, simulations performed at typical loads encountered in tyre applications - $\sigma_{0}=250 \mathrm{kPa}$ - have to include temperature effects for a meaningful description of the friction process. In the following simulations, the surface roughness - and a fortiori the hysteresis friction coefficient $\mu_{H}-$ is systematically modelled with two-scaling-regimes approach. 
The influence of the normal force on the hysteresis friction is first separately investigated for a carbon black filled S-SBR 5025 sliding over a rough granite surface at a constant temperature. By increasing the load, the rubber is able to fill cavities of the rough profile at smaller length scales. This is illustrated by the right diagram in Figure 5.30 where the minimal length scale $\lambda_{\min }$ is found to significantly decrease over the whole range of sliding velocity. At the same time, since the rubber explores a wider contact interval associated with a fractal dimension D $>2$, the contact area is expected to increase. This remark is corroborated by the left diagram in Figure 5.30 where the amount of contact patches varies with the load.
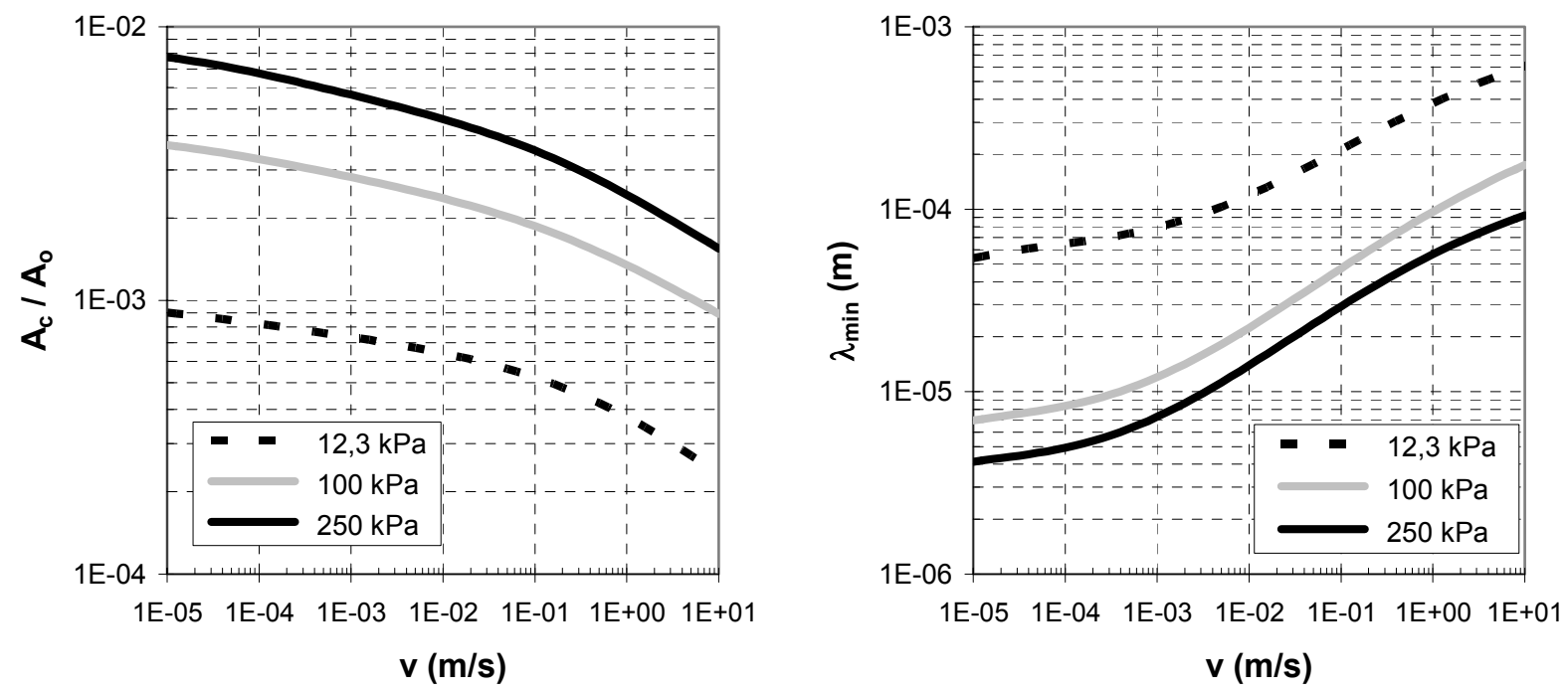

Figure 5.30: Simulated real area of contact (left) and minimal cut-off length (right) for a carbon black filled S-SBR $\mathbf{5 0 2 5}$ on rough granite at different loads

Next, an empirical temperature law was implemented in the friction model. It is partly based on experimental friction results carried out on the same carbon black filled S-SBR 5025 at high sliding velocities: through a thermo-camera, the evolution of contact temperature was followed during stationary friction tests performed on a silicon carbide surface at a load $\sigma_{o}=250 \mathrm{kPa}$. Results revealed average temperatures on the rubber surface of $\mathrm{T}=60^{\circ} \mathrm{C}$ and $80^{\circ} \mathrm{C}$ at sliding velocity $\mathrm{v}=1 \mathrm{~m} / \mathrm{s}$ and $3 \mathrm{~m} / \mathrm{s}$, respectively [76][86]. The temperature profile was adopted and fitted for this particular load by an empirical function. Further results obtained from ABS-Braking tests confirmed the temperatures measured on tread compounds [73][74]. Beside these basic assumptions, it is assumed that the temperature increase is proportional to the load [84]. The resulting empirical load and velocity dependent temperature is shown in Figure 5.31. It allows a systematic investigation of the role played by temperature within the range of high loads and for sliding velocities above $v=1 \mathrm{~cm} / \mathrm{s}$. 


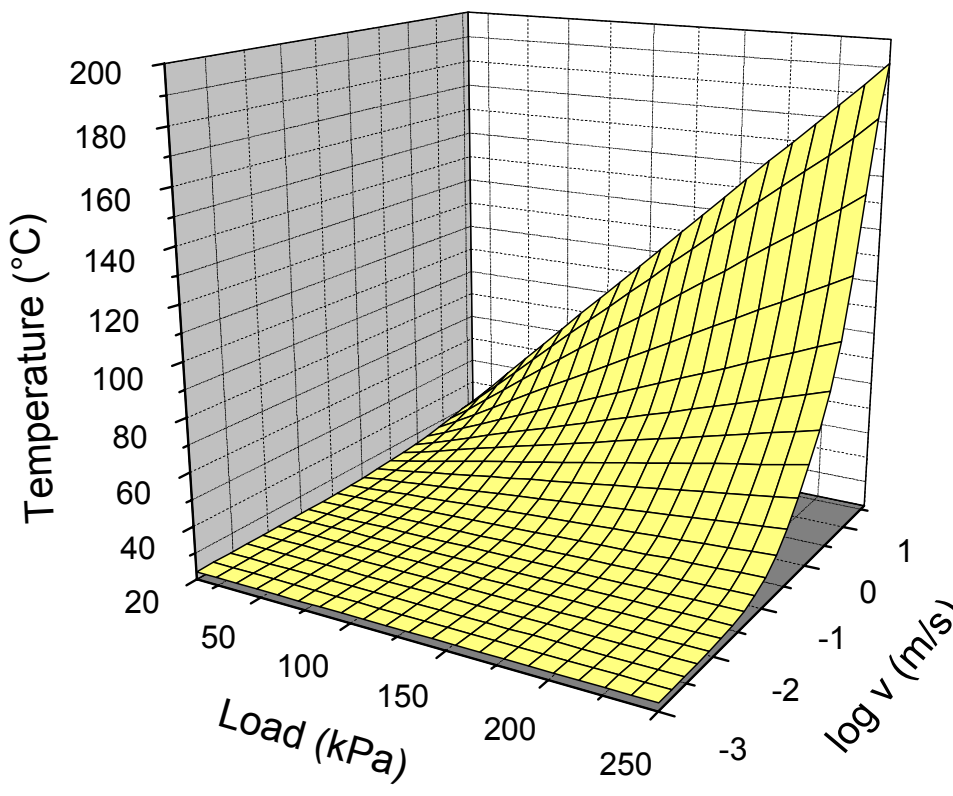

Figure 5.31: Empirical temperature field $T\left(v, \sigma_{0}\right)$ used for simulations at high sliding speeds

Following the empirical temperature profile, simulations of the hysteresis friction coefficient $\mu_{H}$ were performed for carbon black and silica filled S-SBR 5025 on asphalt at a load $\sigma_{o}=250 \mathrm{kPa}$ (Figure 5.32). Prediction with a constant temperature assumed over the whole range of sliding velocity is comparatively shown. Also, the significance of the extended master procedure is highlighted through simulations performed with and without vertical shift factors.
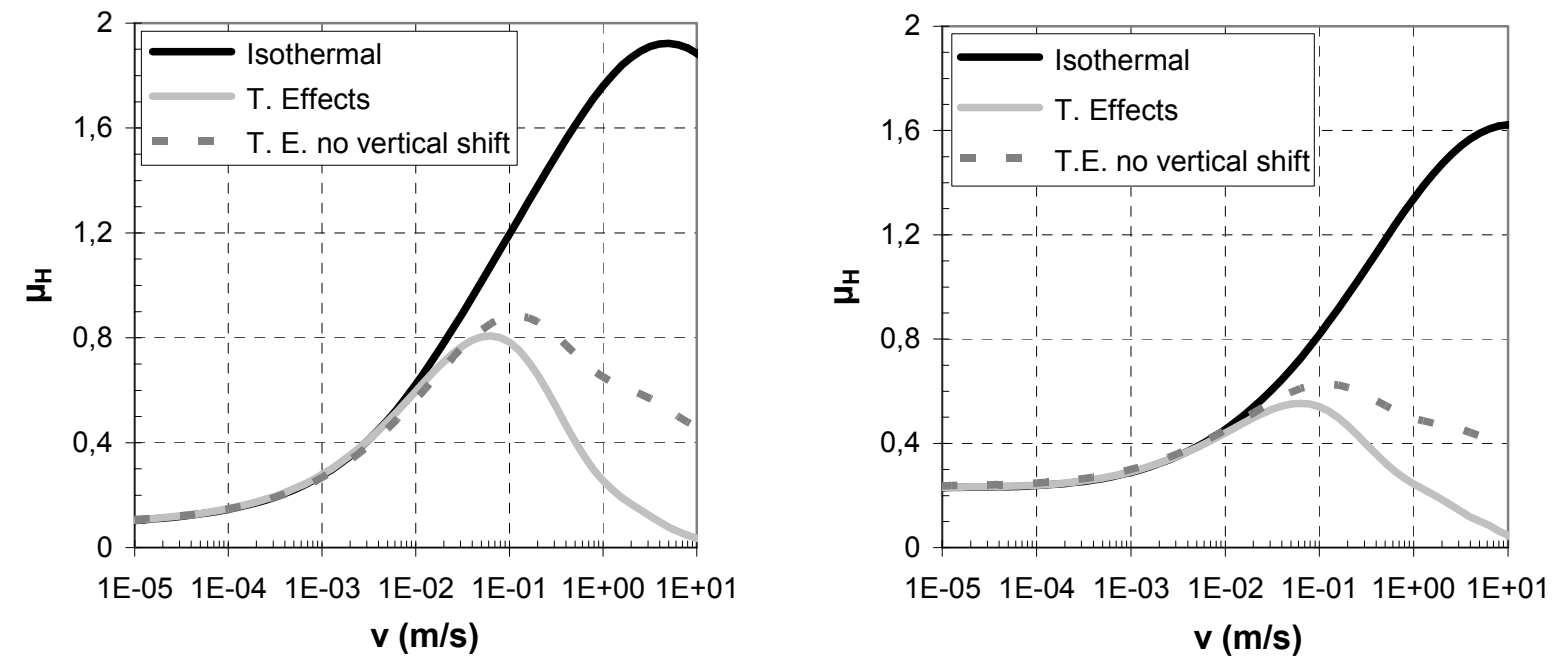

Figure 5.32: Simulated hysteresis friction for silica (left) and carbon black (right) filled S-SBR 5025 on asphalt with/without temperature effects (separate contribution from vertical shift factors). Load $\sigma_{\circ}=250 \mathrm{kPa}$ 
By taking into account the increase of contact temperature above $v \sim 1 \mathrm{~cm} / \mathrm{s}$, the simulated hysteresis friction coefficient dramatically reduced at high sliding velocities. The corresponding $\mu_{\mathrm{H}}$-peak is reduced by about $65 \%$ of its value compared to the ideal case with a constant temperature. Furthermore, the friction maximum is found to occur at a significantly lower sliding velocity located around $v=10 \mathrm{~cm} / \mathrm{s}$, which implies a shift of approximately two decades regarding the initial simulation. Physically, the increase of rubber temperature is reflected by the reference temperature at which dynamic moduli are mastered. Thereby, the dynamic glass transition regime is progressively shifted towards high frequencies during the simulation procedure and the filled composites remain within the rubbery state for that particular load. Hence the dramatic decrease and the pronounced shift of the hysteresis peak.

Temperature dependent vertical shift factors introduced during dynamic mechanical analysis characterise the dynamic softening of filled elastomers above glass transition. The left diagram of Figure 5.32 underlines the impact of generalized master procedure on the resulting hysteresis friction coefficient using the temperature distribution presented above. The application of a classical master procedure - with sole horizontal shift factors - leads to an overestimation of the friction combined with a slightly shifted position of hysteresis peak. The thermally activated processes identified at high temperatures results in an additional decrease of the simulations and one obtains friction values twice as low as predicted by a classical approach at a sliding velocity $v \sim 1 \mathrm{~m} / \mathrm{s}$. Therefore, beside the considerations of temperature effects, the dynamic softening of filled elastomers is crucial for the relevance of friction predictions under realistic conditions encountered during ABS-Braking phases.

The impact of temperature on hysteresis friction was explained by referring to the viscoelastic behaviour of rubber. Similarly to results presented in the last section, the frequency interval can be determined as a function of the sliding velocity. Higher contact temperatures allow rubber to explore cavities at smaller length scales which lead to an increase of the upper frequency of the friction integral. This is illustrated in Figure 5.33 (top diagrams) for the same example presented above, whereby one has to keep in mind that temperature varies along the velocity axis. For a better understanding of friction simulations, the influence of temperature is decoupled from the sliding velocity via the time-temperature superposition principle. In that way, a unique picture is obtained for the frequency interval brought back at laboratory temperature. Figure 5.33 (lower diagrams) illustrates how the occurrence of high contact temperatures at high sliding velocities prevents the elastomer from exploring the dynamic glass transition regime. Such a representation offers the possibility to follow the dynamic response of rubber under real contact conditions for a given temperature profile. 

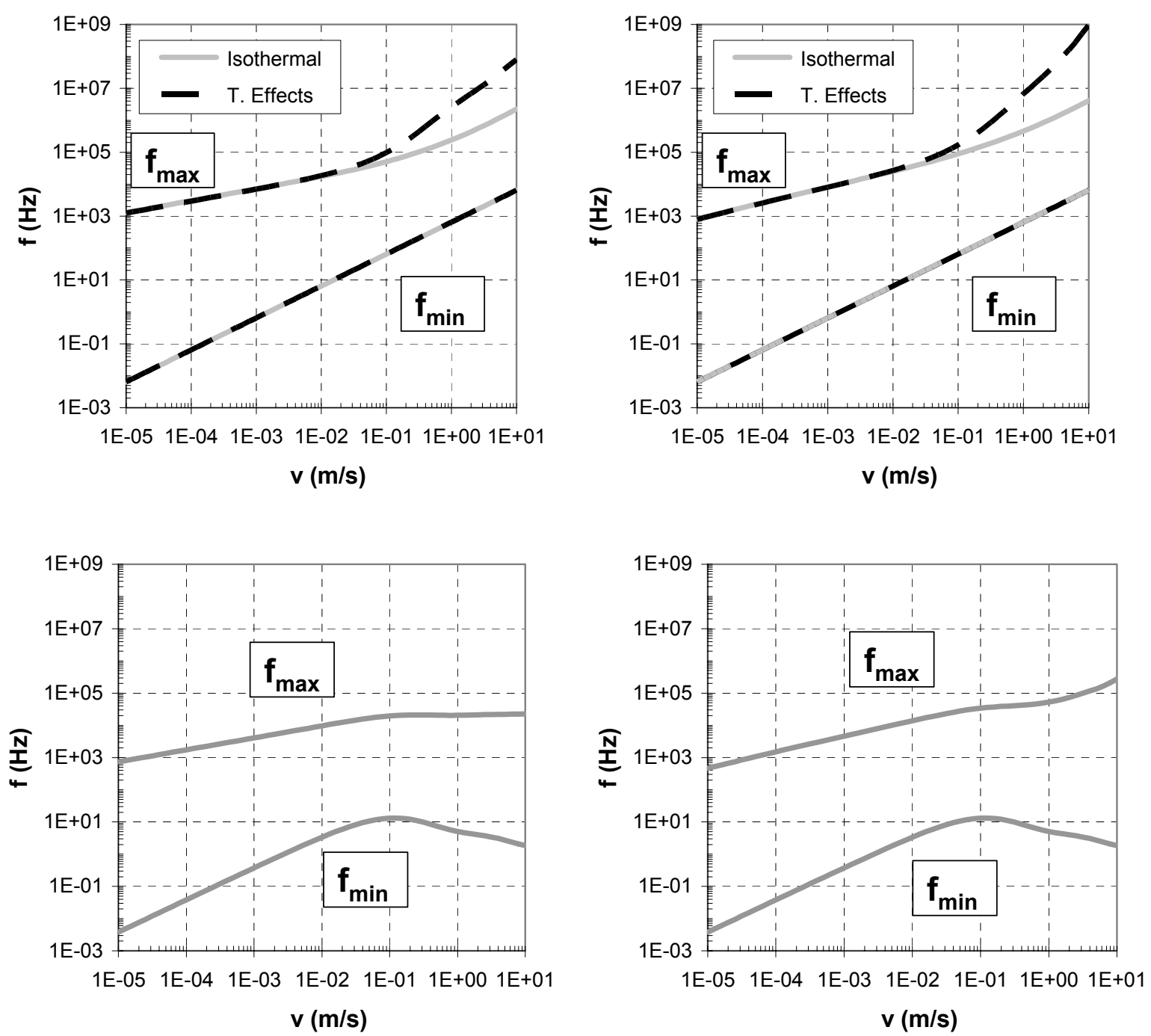

Figure 5.33: Simulated frequency interval contributing to hysteresis friction for silica (left) and carbon black (right) filled S-SBR5025 on asphalt with/without temperature effects (top) and brought back at $T_{\text {ref }}=20^{\circ} \mathrm{C}$ (bottom). Load $\sigma_{\circ}=250 \mathrm{kPa}$

The increase of rubber temperature was seen to widen the contact interval down to micrometer length scales, e.g. the decrease of the real area of contact observed in Figure 5.23 and Figure 5.28 is expected to be compensated by the material softening under real conditions. Figure 5.34 shows the simulated fraction area for carbon black and silica filled SSBR 5025 according to the empirical temperature distribution introduced in this section. Above $v=1 \mathrm{~cm} / \mathrm{s}$, the effect of temperature can be clearly identified and the amount of contact patches is found to increase for both composites. Since thermally activated processes are more pronounced for the carbon black filled compound, the corresponding contact area is found to be higher than for the silica filled S-SBR 5025 above $v \sim 1 \mathrm{~m} / \mathrm{s}$. The dramatic decrease of the hysteresis friction level combined with a marked increase of the contact area clearly indicate that adhesion effects are likely to dominate at high sliding velocities under dry 
contact conditions. Then, the master procedure of filled compounds appears to be a determining step for a relevant description of the friction process under real conditions. More generally, a full treatment of heat transfer arising from sliding process has to include material thermal properties. A recent theory proposes a formulation of the flash temperature distribution for rubber friction on rough surfaces [75].
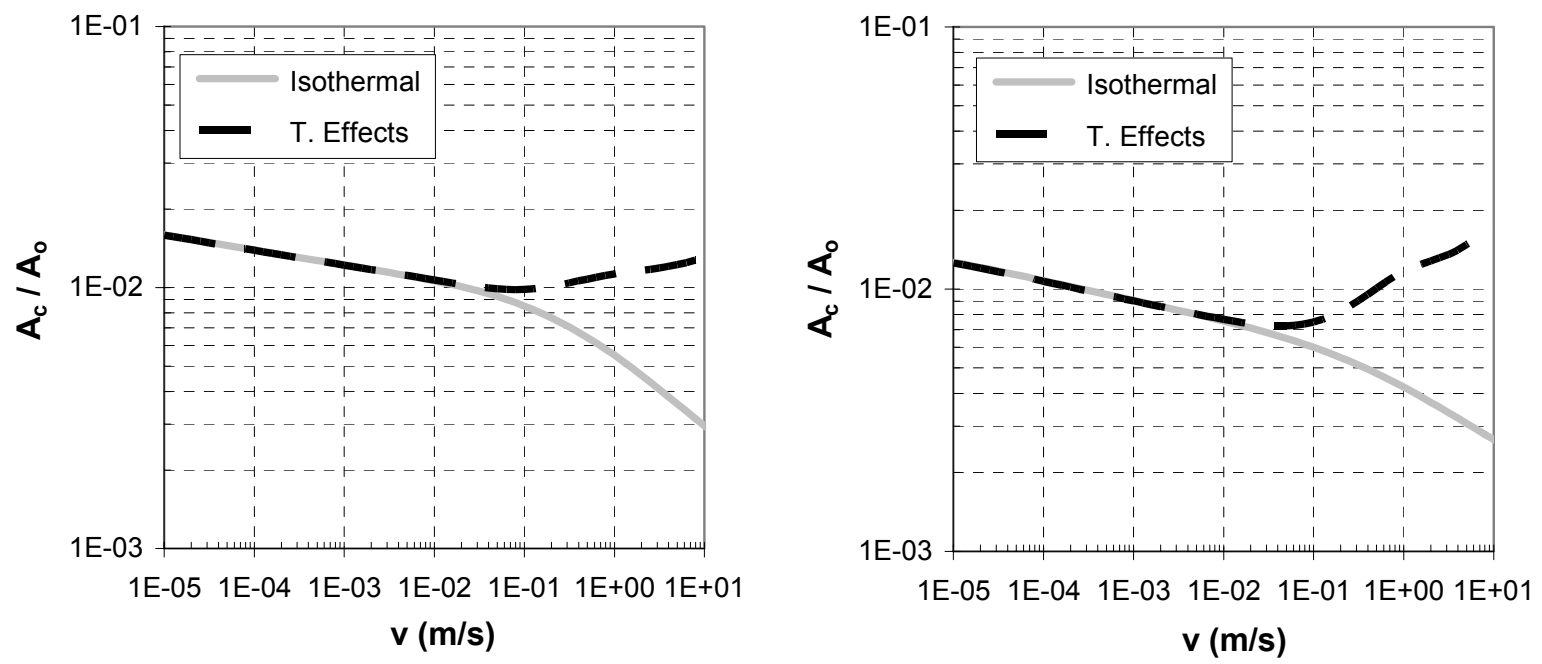

Figure 5.34: Simulated real area of contact for silica (left) and carbon black (right) filled SSBR5025 on asphalt with/without temperature effects. Load $\sigma_{o}=250 \mathrm{kPa}$

Beside temperature effects arising from the material viscoelastic response, the contact is further on examined at various loads. Due to the complex nature of rough surfaces and nonlinear behaviour of elastomers, the load dependence of the rubber friction coefficient can strongly vary if one of both contact partners is exchanged and predictions from Hertz theory are generally not fulfilled for rigid/soft frictional pairings. A closer examination of the hysteresis friction coefficient $\mu_{H}$ shows that a differentiation of both material and surface contributions is possible. Indeed, macroscopic contact parameters evaluated in the frame of a generalized Greenwood-Williamson theory are contained in the front factor. By neglecting the influence of the friction integral on the load dependence of $\mu_{\mathrm{H}}$, one obtains:

$$
\mu_{H} \sim \frac{<z_{p}>}{\sigma_{0}}=\frac{\int_{d}^{\infty}(z-d) \phi(z) d z}{\int_{d}^{\infty}(z-d)^{3 / 2} \phi_{s}(z) d z} \sim \frac{F_{1}(t)}{F_{3 / 2}\left(t_{s}\right)}
$$

where $F_{1}$ and $F_{3 / 2}$ are the Greenwood-Williamson functions introduced in the theoretical section. $t=d / \tilde{\sigma}$ is the normalized distance between rubber and mean value of the profile 
with corresponding standard deviation $\tilde{\sigma}, \tilde{\sigma}_{s}$ accounts for the standard deviation oft the summit height distribution $\phi_{s}(z)$ [65]. At a given sliding velocity, the normalized distance $t$ decreases with increasing load as the rubber is squeezed against the rough substrate. Consequently, the ratio $F_{1} / F_{3 / 2}$ can be used as surface characteristic for predicting the load dependence of $\mu_{H}$ independently of material considerations.

Results are presented in Figure 5.35 for rough granite and asphalt. Simulations were performed at three different loads $\sigma_{0}=60,250$ and $500 \mathrm{kPa}$ within the sliding velocity range $v=10^{-6}-1 \mathrm{~m} / \mathrm{s}$ at laboratory temperature. For each load and by assuming that the contact temperature is constant, an increase of sliding velocity results in high t-values as the rubber is lifted up compared to the mean value of substrate. As expected, the ratio explores the region of lower t-values with increasing load and decreasing sliding velocity. It is worth noticing that the ratio $F_{1} / F_{3 / 2}$ exhibits a continuous master-curve-like path with a complete overlapping over the whole range of simulation parameters. This indicates that load and sliding velocity play an equivalent role from the contact mechanics point of view. It should be noted that the predictor $F_{1} / F_{3 / 2}$ is a sole characteristic of a given rough surface. External factors - rubber type, temperature, load and sliding velocity - only influence the position and the rate of variation on the characteristic curve.
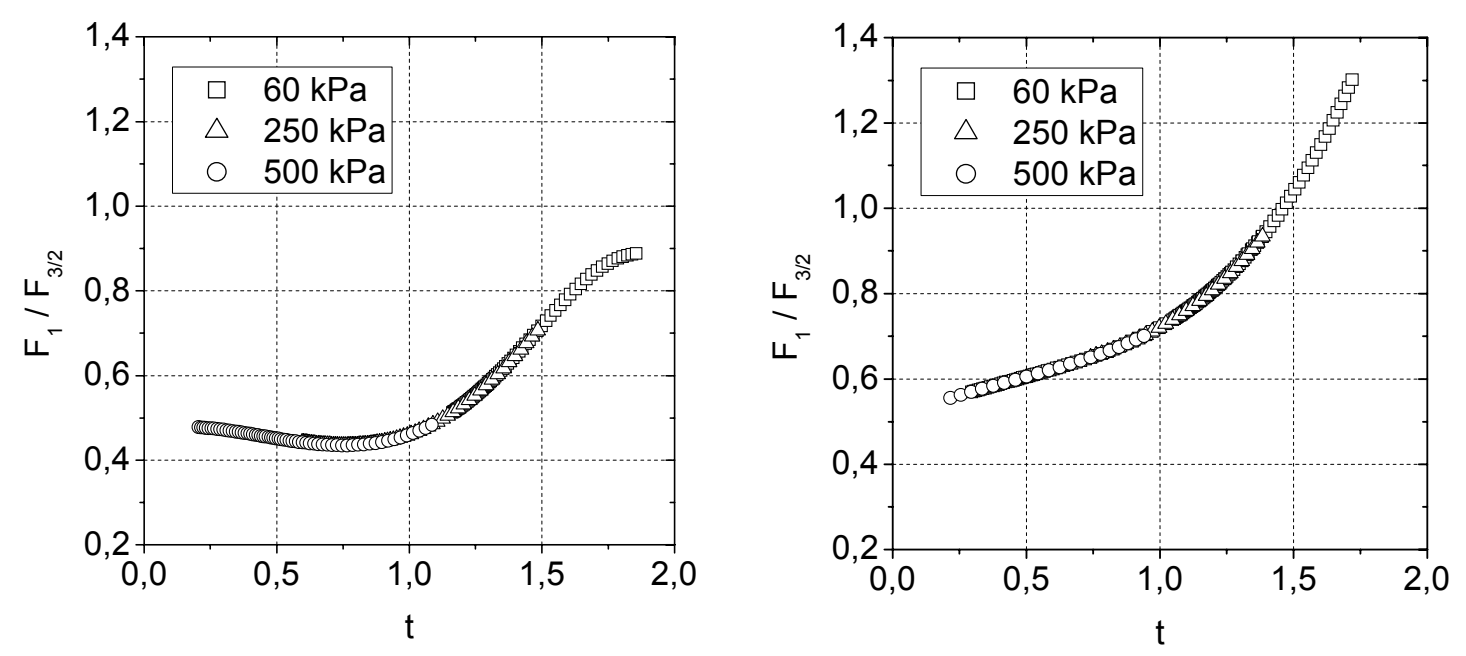

Figure 5.35: Simulated $F_{1} / F_{3 / 2}$ ratio for rough granite (left) and asphalt (right) as a function of $t$ (normalized distance rubber $/$ mean value of rough profile) at three different loads. Interval of sliding velocity: $v=10^{-6}-1 \mathrm{~m} / \mathrm{s}$

Within the range of high t-values, e.g. at low loads or high sliding velocities, the characteristic ratio $F_{1} / F_{3 / 2}$ indicates that the hysteresis friction coefficient $\mu_{H}$ decreases with increasing load. For sufficiently high normal forces and moderate sliding velocities, the load 
dependence of the hysteresis friction coefficient $\mu_{\mathrm{H}}$ is expected to be strongly reduced. This qualitative remark holds for both investigated rough surfaces and also confirms previous studies carried out on various substrates [15]. The difference in roughness amplitude between both surfaces can be indirectly observed on the range of $t$-values as asphalt covers a broader range due to a higher vertical cut-off length $\xi_{\perp}$. The load dependence of the friction integral should be considered via the term associated with macrotexture which mostly contributes to hysteresis friction. The analysis of simulation results shows that the influence of load on the friction integral is negligible over the whole range of sliding velocity above $\sigma_{0} \sim 50 \mathrm{kPa}$, which means that the GW ratio can be used as predictor for the load dependence of the hysteresis friction coefficient under real conditions.

Finally, load dependent simulations of the hysteresis friction coefficient are shown in Figure 5.36 with and without temperature effects for a carbon black filled S-SBR 5025 on asphalt. By assuming a constant contact temperature (left diagram), $\mu_{H}$ is found to decrease over the whole range of sliding velocity with increasing load, which corroborates interpretations made on the GW-ratio curve. If the empirical temperature field of Figure 5.31 is implemented into the simulation procedure, the picture is dramatically changed: indeed, since contact temperatures are assumed to be proportional to the load, the decrease of hysteresis friction is accelerated at high loads. More generally, both increasing and decreasing trends can occur for a given frictional pairing within a sliding velocity sweep [65].
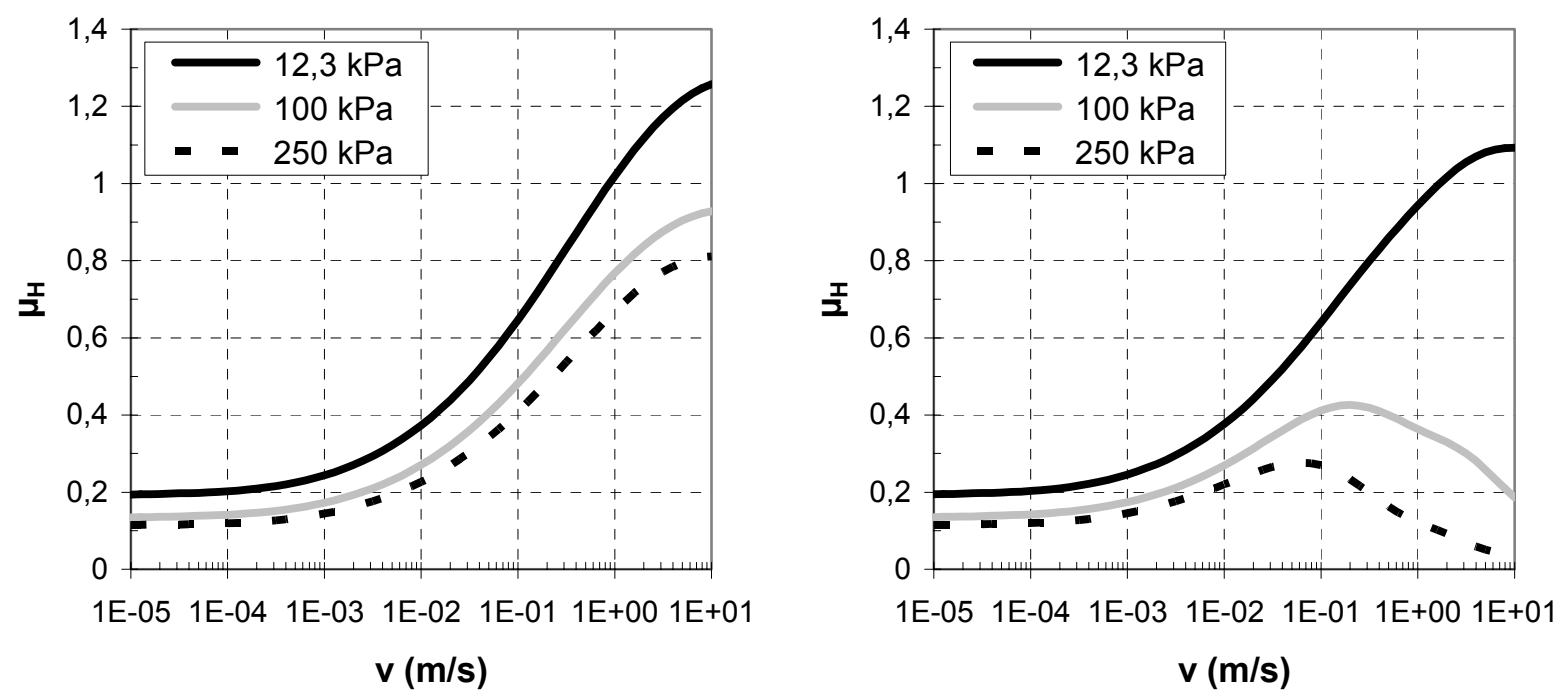

Figure 5.36: Simulated load dependent hysteresis friction for a carbon black filled SSBR5025 on asphalt without (left) and with temperature effects (right) 


\subsection{Correlations with friction measurements - I}

This section presents correlations between experimental friction results and predictions of hysteresis and adhesion friction components. Friction properties of elastomers are first investigated at a moderate load $\sigma_{\circ}=12.3 \mathrm{kPa}$ up to a maximum sliding velocity $v=1 \mathrm{~cm} / \mathrm{s}$, thus limiting the appearance of thermal effects. Also, the systematic use of a solution made of a small amount of detergent mixed with water prevents the occurrence of adhesion effects under wet conditions and allows a direct identification of wet friction results with simulated hysteresis friction. Subsequently, the transition from wet to dry friction is interpreted as adhesion and described by the formulation presented in Chapter 3. Numerical parameters of adhesion and hysteresis friction are listed and discussed in Chapter 6.

\subsubsection{Wet friction results - Hysteresis friction}

The presence of lubricant is of extreme importance for the level of rubber friction on rough surfaces. As previously observed by Grosch, the friction coefficient is experimentally strongly reduced under wet conditions over the whole range of sliding velocities [14]. In particular, the vanishing of the low-velocity adhesion peak indicated that interfacial molecular interactions are hindered by the lubricant film, while the position of the hysteresis maximum remains unchanged. For rough surfaces with sharp summits - typically silicon carbide substrates - a small amount of detergent mixed in a water solution leads to a further decrease of the friction coefficient [14][70]. Since the lubricant viscosity is not significantly affected, the corresponding contribution on the friction force can be excluded. Regarding the roughness, a stabilized interfacial film is likely to cover asperities within the micrometer range. However, as shown earlier in this section, the largest roughness length scales mostly contribute to hysteresis mechanism, so that measurements under wet conditions can be reasonably correlated with simulated hysteresis friction.

Friction tests were carried out according to the experimental procedure presented in Chapter 4. In the following, "wet conditions" means that the rough substrate was completely covered by a water solution mixed with detergent at $5 \%$ concentration. Simulations of hysteresis and adhesion friction were performed on the basis of mechanical data characterised at a dynamic strain amplitude $\varepsilon=3 \%$ and mastered at laboratory temperature $\mathrm{T}=23^{\circ} \mathrm{C}$. Figure 5.37 shows friction results of carbon black and silica filled S-SBR 5025 on rough granite under wet conditions. Both compounds exhibit a continuous increase of the friction coefficient within the measuring range, with a pronounced increase of the silica filled composite. The crossover 
previously observed during mechanical spectroscopy is also found on the friction coefficient, thus confirming the viscoelastic nature of rubber sliding friction on rough surfaces.

The corresponding simulations of hysteresis friction are shown on both diagrams with a differentiation made between the one and two-scaling-regimes approach. By considering a single scaling range, the predicted $\mu_{\mathrm{H}}$-values tend to overestimate the experimental level of wet friction with increasing sliding velocity. For example, the silica filled S-SBR 5025 shows a friction value $\mu=0.7$ measured at a sliding velocity $v=1 \mathrm{~cm} / \mathrm{s}$, while the simulated hysteresis friction accounts for $\mu_{H}=1.1$. A fast calculation shows that if the observed discrepancy is assumed to be solely due to the generation of heat at the interface, one would obtain a rubber temperature $\mathrm{T}=60^{\circ} \mathrm{C}$ which is unlikely to occur at moderate loads and low sliding speeds. The consideration of a second scaling regime leads to a better description of wet friction results for both filled composites. Since the extended modelling of roughness gives a finer description of the largest length scales - which actually mainly contribute to hysteresis friction -, improved correlations with wet friction behaviour can be achieved within the range of low sliding velocities.
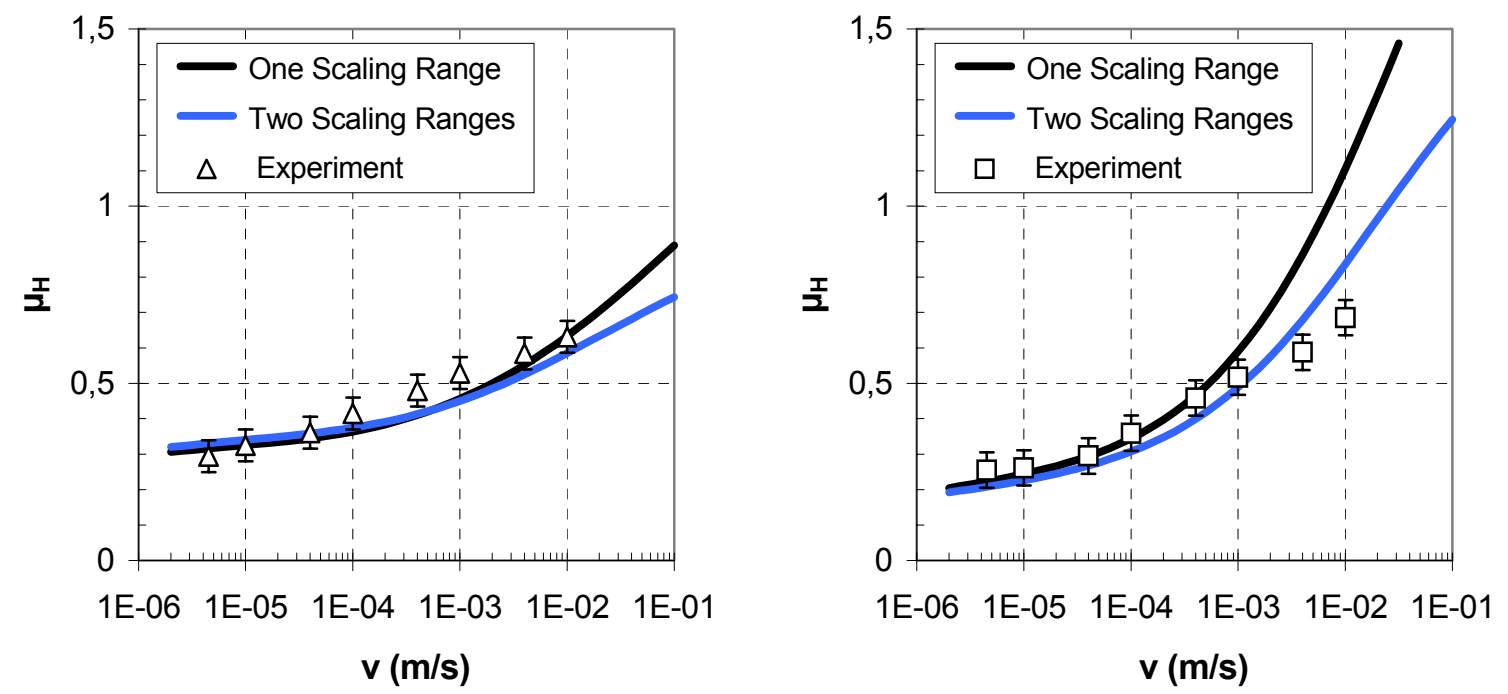

Figure 5.37: Wet friction results and simulated hysteresis friction for carbon black (left) and silica (right) filled S-SBR 5025 on rough granite. Load $\sigma_{o}=12.3 \mathrm{kPa}$

The impact of roughness on wet friction results was investigated via measurements on asphalt carried out under identical experimental conditions. Compared to rough granite, the asphalt surface is characterised by higher roughness amplitude $\xi_{\perp}$ associated with a lower fractal dimension within the micrometer range. According to Equation (3.42), the contact interval $\left[\lambda_{\min }, \xi_{\| 1}\right]$ is therefore expected to increase, and a fortiori the frequency range 
$\left[f_{\min }, f_{\max }\right]$ involved during sliding friction as well. Figure 5.38 shows correlations between theoretical predictions and wet friction results. A similar trend to the one previously observed on rough granite is pointed out, namely a satisfying description of wet friction results by the simulated hysteresis friction at low sliding velocities with characteristic improvement of correlations by using a two-scaling-ranges approach. However, the friction behaviour of the silica filled S-SBR 5025 is clearly overestimated by the model above $v \sim 1 \mathrm{~mm} / \mathrm{s}$, which can not be traced back to the occurrence of temperature effects. The discrepancy found in limited cases is discussed in details in the last section of Chapter 5 , where further extensions of the model are proposed.
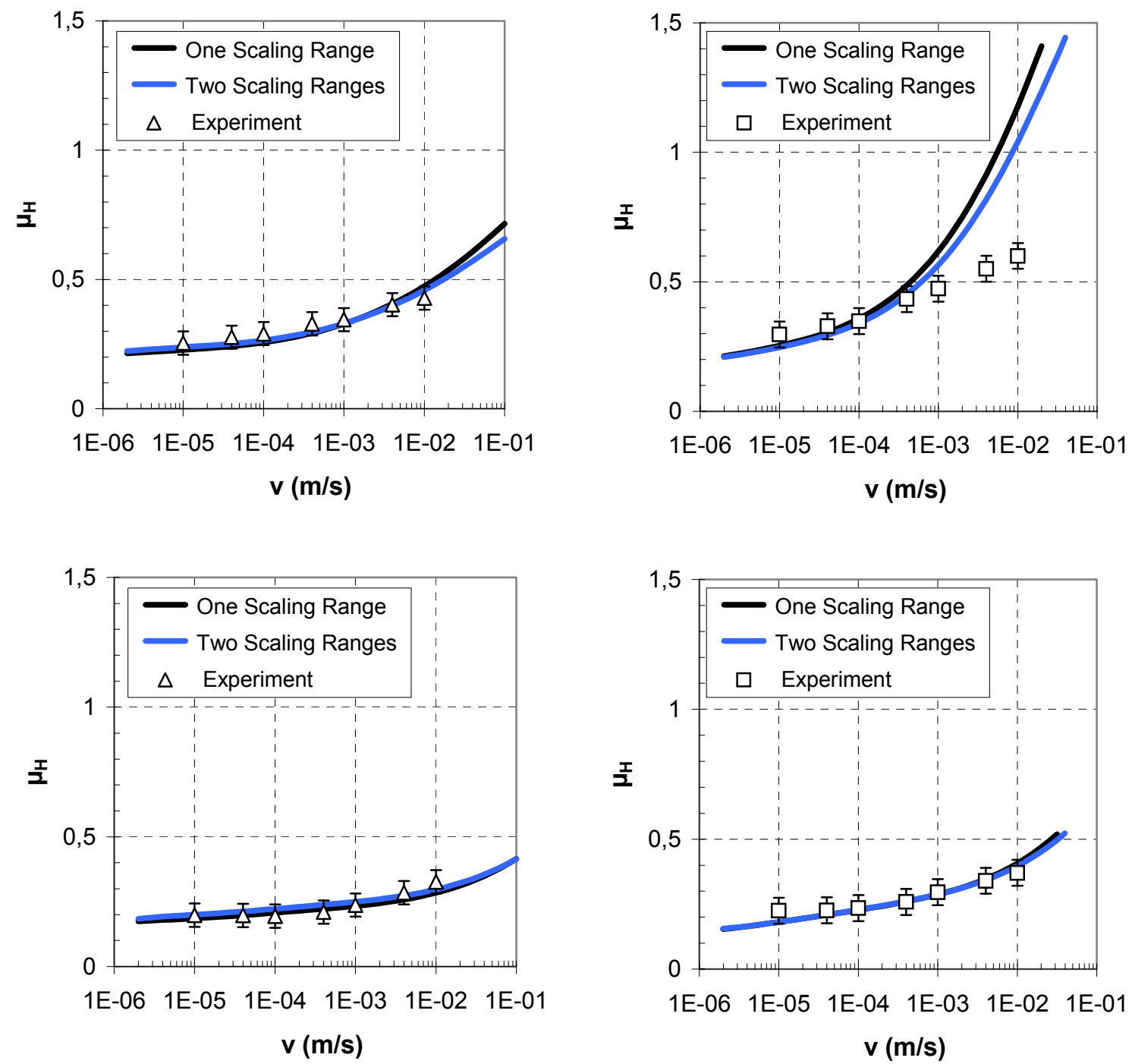

Figure 5.38: Wet friction results and simulated hysteresis friction for carbon black (left) and silica (right) filled S-SBR 5025 (top) and S-SBR 2525 (bottom) on asphalt. Load $\sigma_{0}=12.3 \mathrm{kPa}$ 
In the following, the influence of polymer architecture on friction properties is investigated. The lower graphs of Figure 5.38 show experimental results carried out on silica and carbon black filled S-SBR with $25 \%$ vinyl content at moderate load. As evidenced by relaxation spectroscopy, low vinyl S-SBR is characterised by a shift of the glass transition temperature towards lower temperatures, e.g. higher frequencies. Consequently, the hysteresis friction maximum is expected to occur at higher sliding velocities and the increase rate of $\mu_{H}$ should be thus limited within the measuring range. This is confirmed by measurements shown in Figure 5.38, whereby the theoretical prediction fairly agrees with wet friction results. Since the frequency range contributing to hysteresis friction is located within the rubbery region of S-SBR 2525 composites, the impact of a second scaling regime is therefore more pronounced for filled S-SBR 5025 compounds up to a maximum sliding velocity $v=1 \mathrm{~cm} / \mathrm{s}$.

\subsubsection{Difference dry / wet friction - Adhesion friction}

Since the specific experimental conditions hinder the occurrence of additional effects associated with fluid viscosity or interfacial heat generation during sliding process, the transition from wet to dry friction is interpreted in terms of adhesion. The distinction between adhesion and hysteresis friction has been already discussed in the literature [17]. Physically based considerations of adhesion friction generally involve the periodic formation and breakage of molecular bonds between polymer chains and a substrate during sliding process [13][28][33]. As a result, rubber adhesion is found to exhibit viscoelastic features with characteristic length scales located below the micrometer range, thus indicating that molecular mechanisms come into play.

The present work proposes a multi-scale formulation of dynamic contact problems for rubbery materials sliding over rough surfaces. Thereby, hysteresis friction and contact parameters like the real area of contact are determined through surfaces descriptors and dynamic properties of the bulk material, while the modelling of adhesion includes the velocity dependent interfacial shear strength describing the kinetics of peeling effects at the interface. Thus, even if both friction components are driven by the material viscoelastic response during the sliding process, the corresponding physical mechanisms fundamentally differ from the length scale at which they occur. In the following, the relevance of adhesion concepts is systematically investigated, in particular the simulated real area of contact and interfacial shear strength with respect to material and surface effects.

Figure 5.39 shows wet and dry friction results for carbon black and silica filled S-SBR 5025 on rough granite. The role of hysteresis friction and the implications of an extended approach 
for the description of roughness scaling properties were already highlighted in Figure 5.37. The dry friction coefficient is found to be constant over the range of measurement with a slight decrease at very low sliding velocities. The silica filled composite shows a higher friction level compared to the carbon black filled system $(\Delta \mu \sim 0.3)$, which accordingly indicates that the adhesion component is significantly higher for the former. By following the approach used for separating hysteresis from adhesion effects on rough surfaces, adhesion friction appears to be the main contribution to rubber friction under dry conditions.
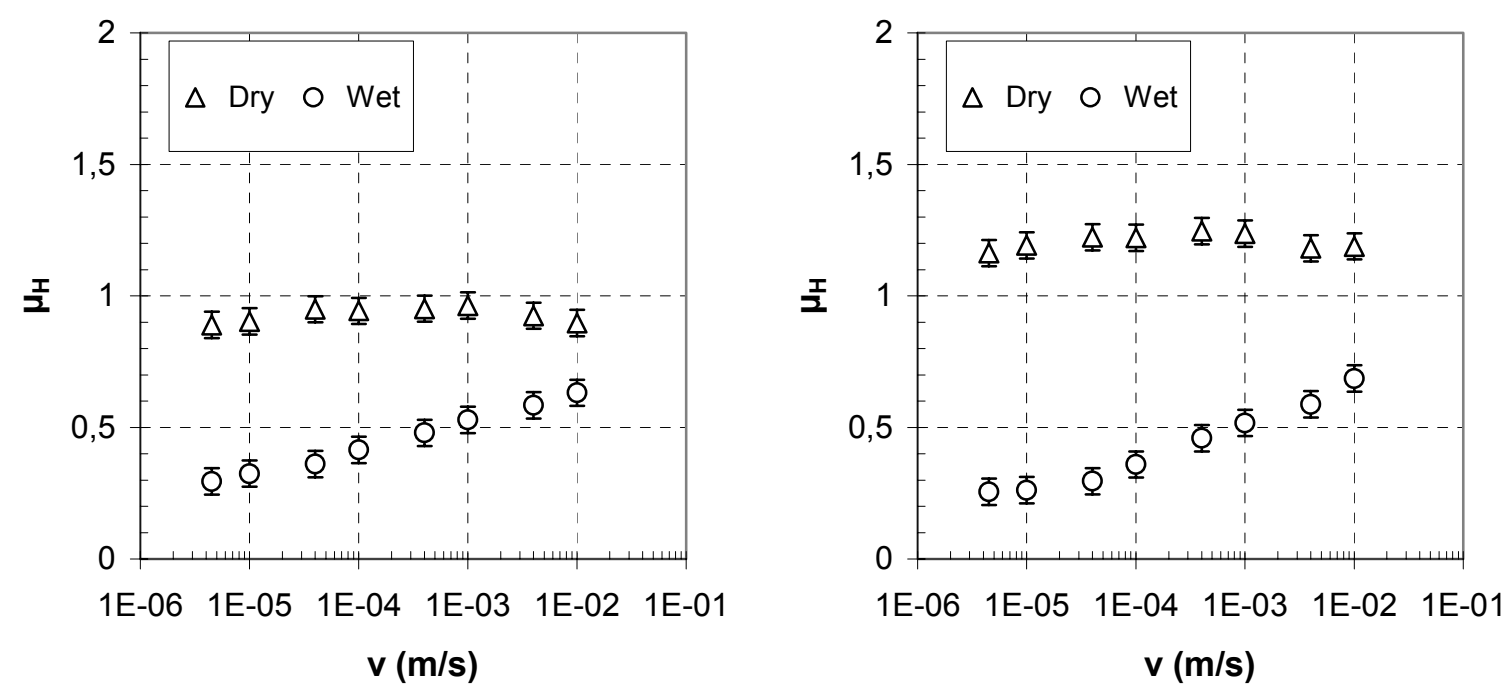

Figure 5.39: Wet and dry friction results for carbon black (left) and silica (right) filled S-SBR5025 on rough granite. Load $\sigma_{o}=12.3 \mathrm{kPa}$ (from [66])

Figure 5.40 shows the experimental difference $\Delta \mu=\mu_{\text {dry }}-\mu_{\text {wet }}$ obtained between dry and wet friction results on rough granite for both filled S-SBR 5025 composites at low sliding velocity. As previously indicated in Figure 5.39, the silica filled compound is characterised by a higher level of adhesion within the range of measurement. By referring to Equation (3.47), the interfacial shear strength $\tau_{\mathrm{s}}$ was first assumed as constant while the roughness was modelled with a one-scaling-regime approach. Obviously, experimental data can be well adapted with the predicted adhesion friction coefficient depicted with dashed lines, thus demonstrating that a proper description of rubber friction on dry surfaces is possible with Equation (3.47) for filled S-SBR 5025 composites. Accordingly, the decreasing adhesion component is fully predicted by the simulated real area of contact reflecting an increase of material dynamic stiffness at high sliding velocity. The examination of the interfacial shear strength $\tau_{\mathrm{s}}$ shows no significant variation which could explain the different levels of adhesion friction: indeed, one obtains $\tau_{\mathrm{s}}=13.8 \mathrm{MPa}$ and $13.2 \mathrm{MPa}$ for the silica and carbon black filled composite, respectively. Hence, this confirms that the level of adhesion friction for filled S-SBR 5025 is primarily governed by the real area of contact found to be significantly larger for the silica 
reinforced composite. This remark holds for a two-scaling-regimes approach where the interfacial shear strength is found to be smaller due to an increase of the real area of contact (Figure 5.28). At the same time, the kinetics of peeling effects can be assumed as constant over the range of measurements, e.g. down to a sliding velocity $v=10^{-5} \mathrm{~m} / \mathrm{s}$. Below this sliding velocity, the discrepancy between adhesion prediction and friction results indicates that the velocity dependence of the interfacial shear strength $\tau_{\mathrm{s}}$ should be taken into account for the formulation of adhesion friction. By referring to the concepts introduced in Chapter 3 , the critical velocity of the interfacial shear strength is numerically found to be located at $\mathrm{v}_{\mathrm{c}} \sim 6.10^{-6} \mathrm{~m} / \mathrm{s}$ and $\mathrm{v}_{\mathrm{c}} \sim 2.10^{-6} \mathrm{~m} / \mathrm{s}$ for the silica and carbon black filled S-SBR 5025, respectively.

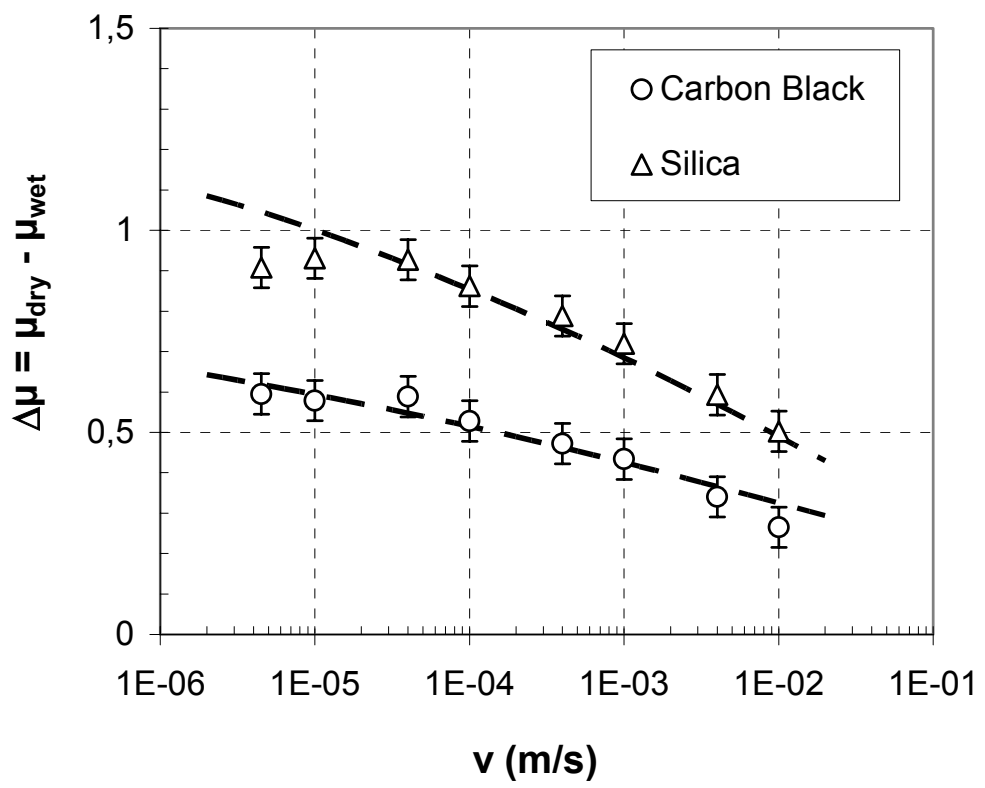

Figure 5.40: Difference between wet and dry friction results for carbon black and silica filled S-SBR5025 on rough granite. Dashed lines: scaled area of contact with $\tau_{\mathrm{s}}=13.8 \mathrm{MPa}$ and $13.2 \mathrm{MPa}$ for silica and carbon black, respectively (from [66])

Experimental results indirectly highlight the relevance of the proposed master procedure applicable to filled and unfilled elastomers. In particular, the temperature dependence of the vertical shift factors and the resulting simulated contact parameters can be traced back to be occurrence of thermally activated processes at the vicinity of filler particles. The favourable inter-locking behaviour of silica filled composites on rough surfaces is fully predicted by a multi-scale approach of dynamic contact and experimentally confirmed in the range of low sliding velocities. This is confirmed by friction results carried out on rough granite for filled SSBR 2525 composites shown in Figure 5.41. Since the corresponding glass transition is located at a lower temperature, e.g. higher frequency, the increase of wet friction is limited within the range of measurement while the hysteresis friction maximum occurs around 
$\mathrm{v} \sim 100 \mathrm{~m} / \mathrm{s}$. Wet friction data are fairly described by the simulation independently of the number of scaling regimes. The glass transition temperature is seen to dramatically influence the level of dry friction at constant filler content, whereby an increase of the friction coefficient is observed up to $v \sim 2 \mathrm{~mm} / \mathrm{s}$ for both composites, corresponding to the position of the adhesion maximum - and approximately the critical velocity (see Table 6.1). The shift of the dry friction plateau illustrates the viscoelastic nature of rubber friction on rough surfaces while the level of adhesion confirms improved interlocking properties of silica filled S-SBR composites physically related to temperature activated processes leading to favourable dynamic behaviour above glass transition.
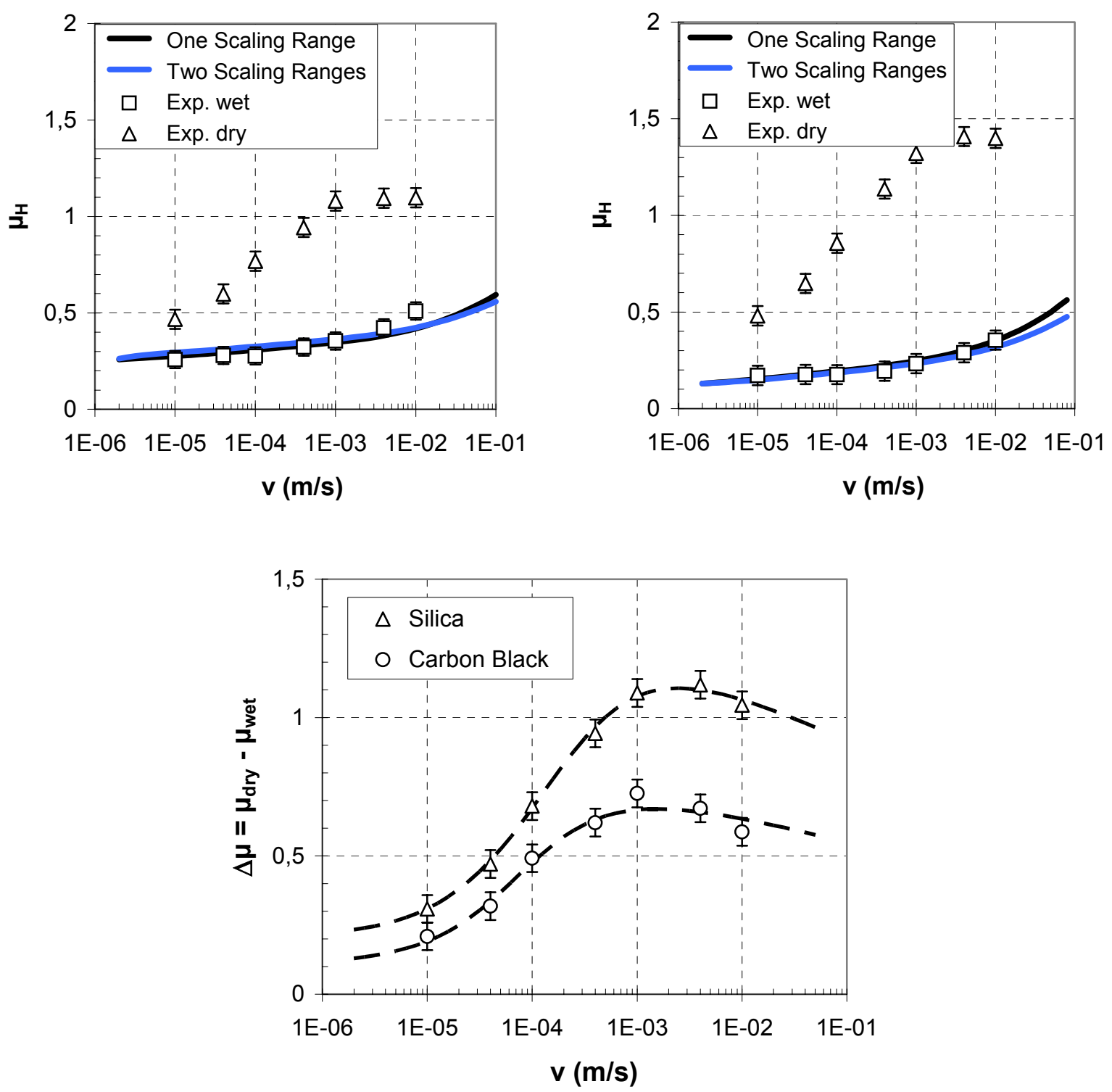

Figure 5.41: Wet and dry friction results (top) for carbon black (left) and silica (right) filled S-SBR 2525 on rough granite with corresponding simulated hysteresis friction. Bottom: Experimental difference dry/wet with simulated adhesion component (dashed lines). Load $\sigma_{o}=12.3 \mathrm{kPa}$ 
To complete this study on rough granite, friction results are presented in Figure 5.42 for a carbon black filled E-SBR. As previously observed on the low- $T_{g} S-S B R$, the experimental wet friction coefficient is found to slightly increase over the range of measurements as a consequence of the shift of the dynamic glass transition (Figure 5.17). Since the frequency interval involved during sliding process is located within the rubbery region over the measuring range, the simulated hysteresis friction is not expected to strongly vary with increasing sliding velocity. This prediction is fairly reproduced by the experimental results. Dry friction values significantly increase with sliding velocity up to $v=1 \mathrm{~cm} / \mathrm{s}$ and indicate that the adhesion peak occurs within the range of measurements. This is evidenced by the adhesion plot in Figure 5.42 (right diagram) where the adhesion maximum is found around $v=1 \mathrm{~mm} / \mathrm{s}$ as observed for the carbon black filled S-SBR 2525.
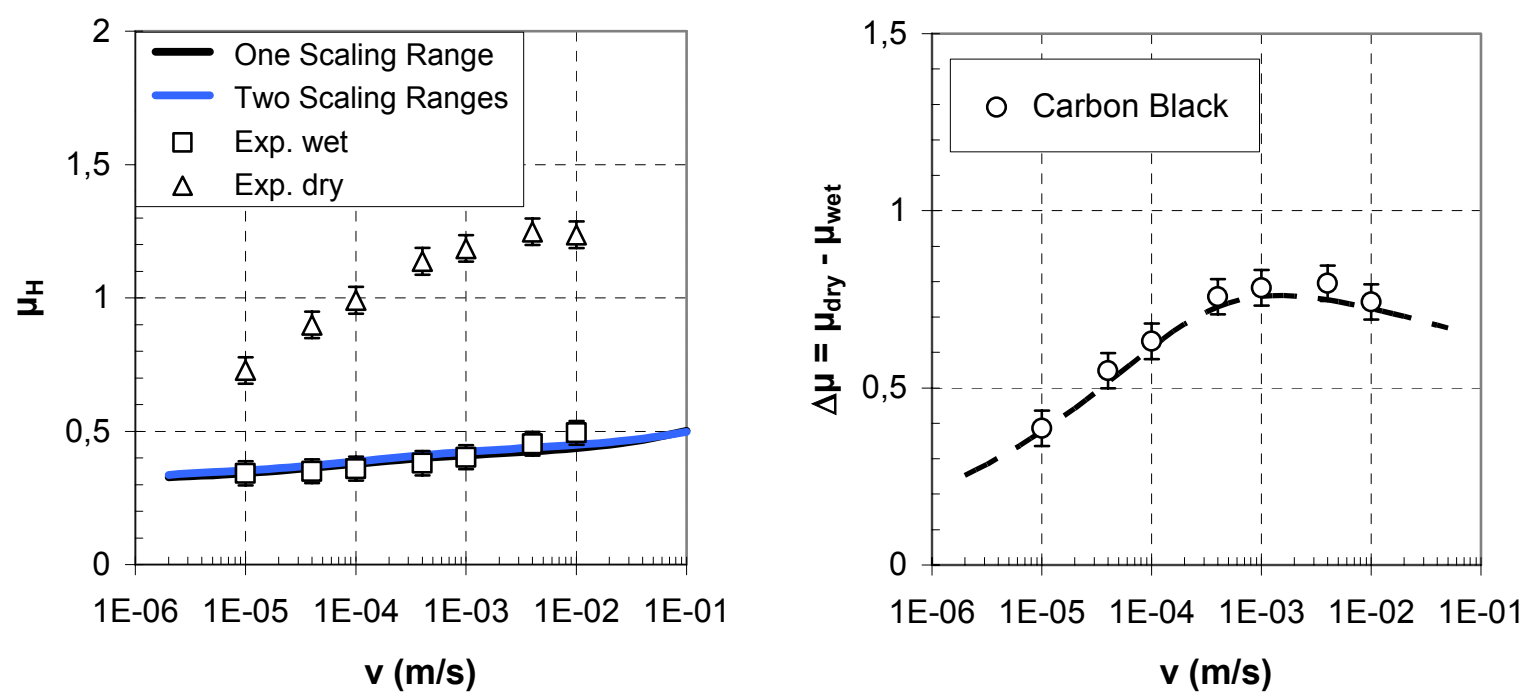

Figure 5.42: Wet and dry friction results for carbon black filled E-SBR 1500 on rough granite with corresponding simulated hysteresis friction (left) and experimental difference dry/wet (right) with simulated adhesion component (dashed line). Load $\sigma_{o}=12.3 \mathrm{kPa}$

Next, correlations of hysteresis and adhesion friction gained from experimental results on rough granite are depicted up to the range of high sliding velocity with a two-scaling-ranges approach. Simulations are shown without including temperature effects, this approach being basically equivalent to the one applied by Grosch, whereby friction master curves were constructed through friction measurements at various temperatures in the range of low sliding velocity [13][16]. In particular, the friction maximum of filled composites is significantly reduced with increasing filler content under dry conditions and goes through a plateau maximum distributed over several decades of sliding velocity. Though the decrease of the friction peak is generally correlated with the one observed on the frequency dependent loss 
factor, the progressive saturation of the friction maximum indicates that an additional mechanism contributes to dry sliding friction, namely adhesion.

This is schematically shown in Figure 5.43 for carbon black filled S-SBR 5025 on rough granite. Simulated hysteresis and adhesion friction are extrapolated according to correlations gained from friction measurements at low sliding velocities. Since the rough granite surface shown in Figure 5.37 and Figure 5.39 differs from the one used in the present work, the previous results should therefore not be directly compared to the correlations depicted in Figure 5.43 [66]. Due to the introduction of a second scaling regime, the increase of hysteresis friction is limited up to a maximum $\mu_{H \max }=0.7$ reached at a sliding velocity $\mathrm{V}_{\mathrm{H}}=0.5 \mathrm{~m} / \mathrm{s}$. The adhesion friction goes through a peak associated with a critical velocity $\mathrm{v}_{\mathrm{c}}=10^{-5} \mathrm{~m} / \mathrm{s}$ and further decreases with increasing sliding velocity. Within the range $\left[\mathrm{v}_{\mathrm{c}}, \mathrm{v}_{\mathrm{H}}\right]$, the decrease of adhesion friction combined with the increase of hysteresis friction leads to a constant level of dry friction illustrated by a broad maximum plateau.

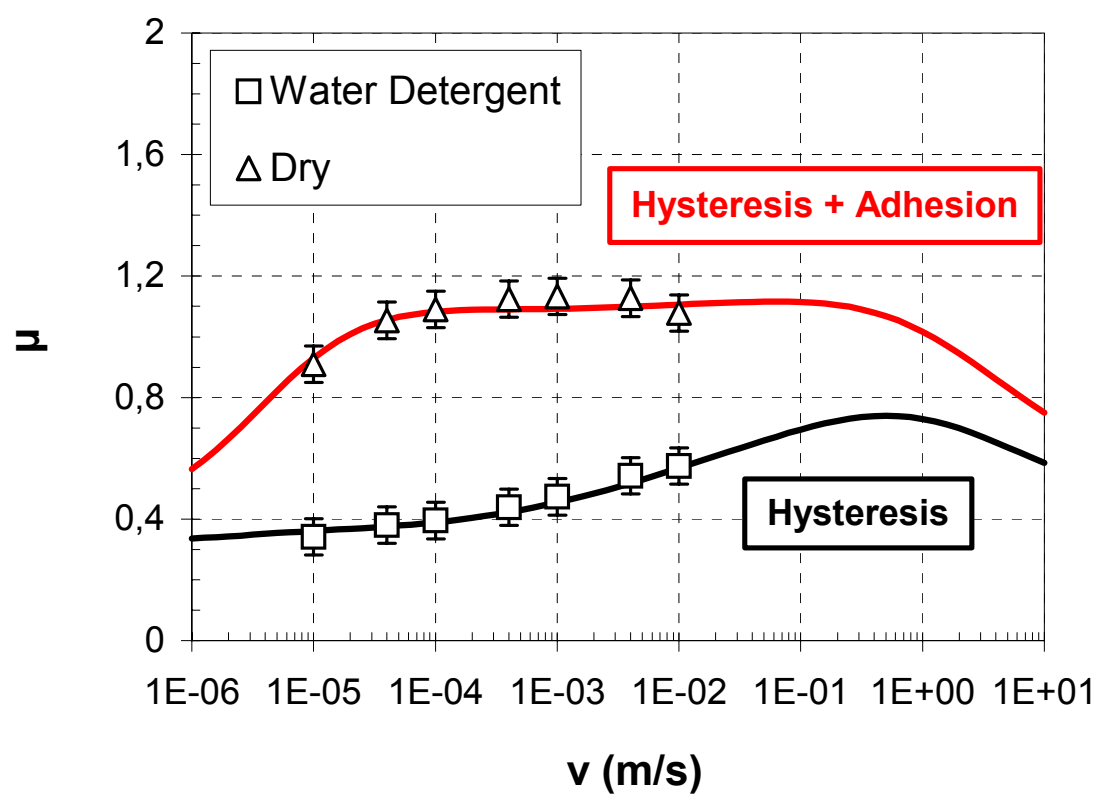

Figure 5.43: Extended correlations of hysteresis and adhesion friction under wet and dry conditions for a carbon black filled S-SBR 5025 on rough granite

By referring to previous experimental observations, the broad saturation of dry friction for filled elastomers on rough surfaces is fully predicted by a multi-scale modelling sliding friction for soft / rigid frictional pairings [13][16]. The corresponding formulation of hysteresis and adhesion friction on rough surfaces allows a deeper comprehension of the physical mechanisms involved during sliding process. Hysteresis friction arises from the rubber viscoelastic response caused by the mechanical excitation of asperities at various length scales and accordingly basically depends on dynamic properties of the bulk elastomer. 
Adhesion is associated with two antagonist mechanisms: first the real contact area down to the micrometer range which reflects the material dynamic hardening with increasing sliding velocity. Secondly, the interfacial shear strength $\tau_{\mathrm{s}}$ is related to the kinetics of periodical formation and breakage of molecular bonds within the contact area and basically increases with increasing sliding velocity up to a critical velocity $v_{c}$.

The same procedure was applied to the carbon black filled E-SBR composite on rough granite. Hysteresis and adhesion friction simulations were extended according to correlations shown in Figure 5.42. As expected, characteristic features of both friction components are shifted towards higher sliding velocities. While the hysteresis friction maximum $\mu_{H}=1.0$ is located at a sliding velocity $\mathrm{V}_{\mathrm{H} \max }=100 \mathrm{~m} / \mathrm{s}$, the critical velocity of the adhesion component is found at $v_{c}=3.10^{-4} \mathrm{~m} / \mathrm{s}$. Following the same argumentation, the addition of decreasing adhesion and increasing hysteresis leads to an almost constant dry friction coefficient over many decades of sliding velocity. This confirms experimental results, whereby the friction plateau was found to strongly depend on the glass transition temperature of the basis polymer [13][16].

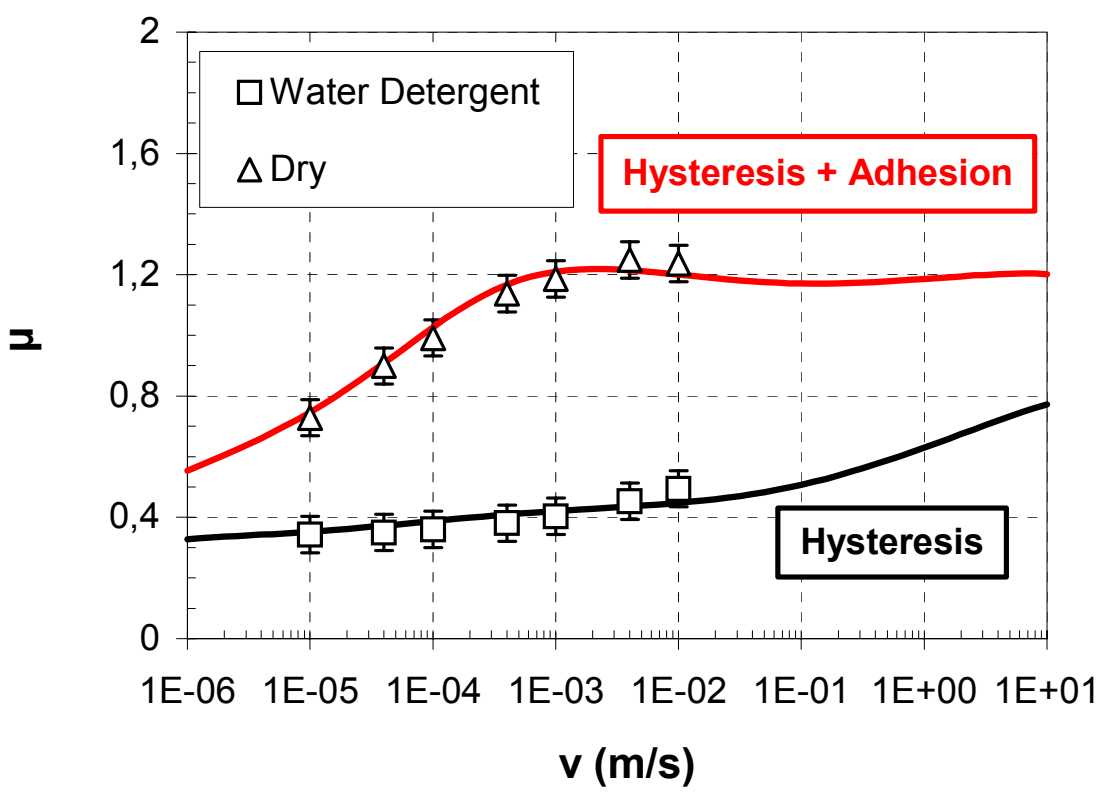

Figure 5.44: Extended correlations of hysteresis and adhesion friction under wet and dry conditions for a carbon black filled E-SBR 1500 on rough granite

Accordingly, the adhesion component mostly contributes to dry friction at moderate load and within the range of low sliding velocity. Under real conditions, since the occurrence of flash temperatures leads to a dramatic reduction of hysteresis friction combined with an increase of the contact area (Figure 5.32 and Figure 5.34), one can reasonably expect the predominance of the adhesion contribution under dry and clean contact conditions. 
Therefore, the formation of contact patches over several length scales appears to be a crucial factor for the optimization of grip properties under dry conditions. For wet surfaces, the drop of adhesion has to be compensated by enhanced material losses in the highfrequency region in order to guarantee a sufficient level of friction within the contact area.

Finally, the impact of surface roughness on adhesion is discussed on the basis of friction measurements carried out on asphalt for carbon black and silica filled S-SBR composites. The roughness analysis of asphalt already showed significantly different scaling behaviour compared to rough granite with a smaller fractal dimension of microtexture. According to Equation (3.42), one expects a broader contact interval $\left[\lambda_{\min }, \xi_{\| 1}\right]$ and a higher contact area $A_{c}$. Also, the difference in the morphological descriptors should affect the level of adhesion and the corresponding position of critical velocity.

Figure 5.45 shows friction results carried out for silica and carbon black filled S-SBR 5025 on asphalt under dry conditions (left diagram). The corresponding wet friction data and hysteresis friction simulations were already presented in Figure 5.38. As previously observed on rough granite for filled S-SBR 5025 composites, the level of dry friction is strongly influenced by filler type with higher values found for the silica filled composite.
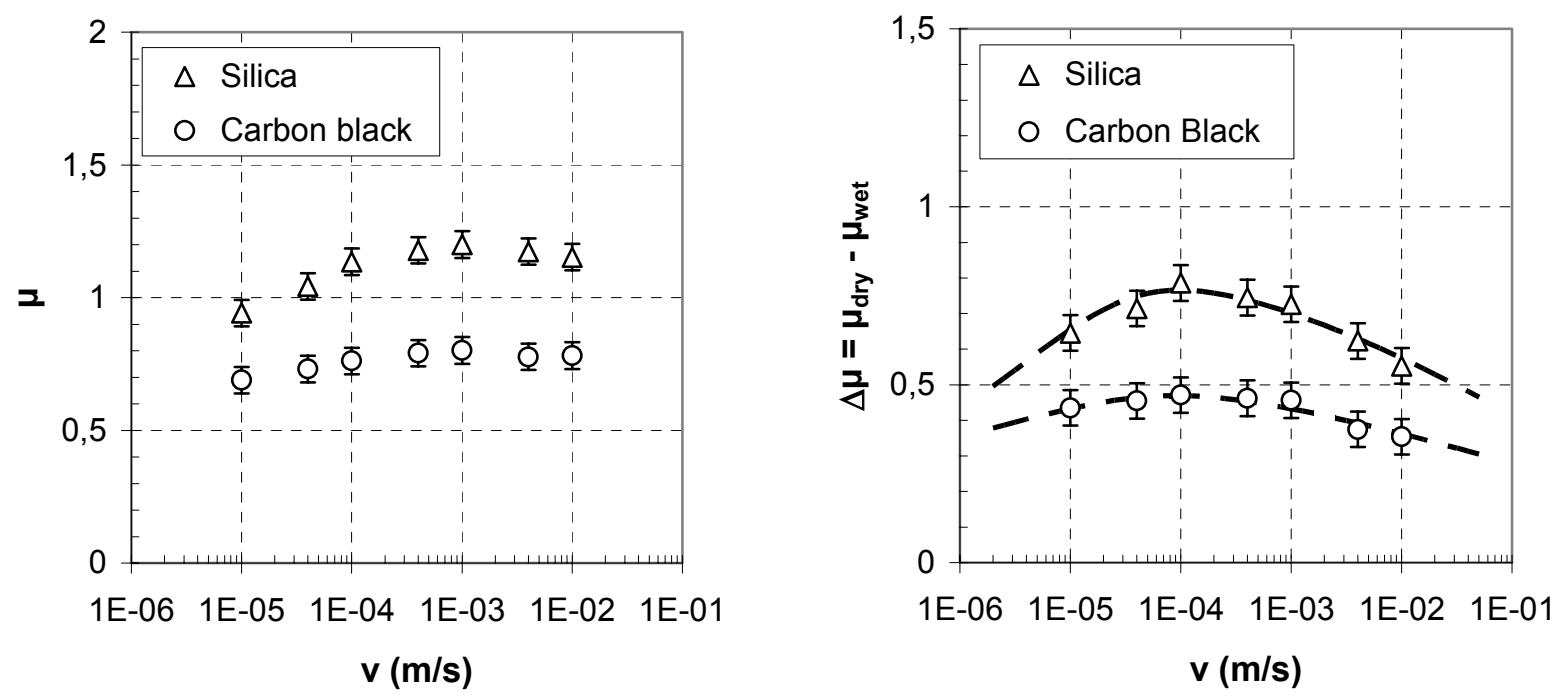

Figure 5.45: Dry friction results (left) and experimental difference dry/wet (right) with simulated adhesion component (dashed line) for silica and carbon black filled S-SBR 5025 on asphalt. Load $\sigma_{o}=12.3 \mathrm{kPa}$

The right diagram of Figure 5.45 shows the difference $\Delta \mu$ between dry and wet friction results with the corresponding simulation of adhesion friction. Compared to results obtained on rough granite, the adhesion component is not found to continuously decrease over the range 
of measurement, but rather goes through a maximum around located around $\mathrm{v} \sim 10^{-4} \mathrm{~m} / \mathrm{s}$. While the decrease of $\Delta \mu$ is clearly attributed to an effect of the real area of contact, the early increase visible below $v \sim 10^{-4} \mathrm{~m} / \mathrm{s}$ indicates that the kinetics of peeling effects actually contributes to adhesion friction at very low sliding velocity. A fitting procedure gives $v_{c}=3.10^{-5} \mathrm{~m} / \mathrm{s}$ and $v_{c}=6.10^{-5} \mathrm{~m} / \mathrm{s}$ for carbon black and silica filled S-SBR 5025, respectively. Similar to rough granite, the position of $\mathrm{v}_{\mathrm{c}}$ for the silica filled S-SBR 5025 is found at a slightly higher sliding velocity than the carbon black reinforced one. Concerning the level of adhesion, no significant difference can be established between both surfaces. Therefore, the surface roughness mostly influences the position of the adhesion maximum and the corresponding critical velocity $\mathrm{v}_{\mathrm{c}}$ for filled S-SBR 5025 composites.

The examination of dry and adhesion friction results for filled S-SBR 2525 composites on asphalt confirms previous features observed on rough granite, namely a continuous increase of the dry friction coefficient and an adhesion maximum located within the range of measurement (Figure 5.46). Also, superior inter-locking properties of silica filled S-SBR composites are illustrated by a higher adhesion component above the critical velocity located at $\mathrm{v}_{\mathrm{c}}=7.10^{-4} \mathrm{~m} / \mathrm{s}$ and $\mathrm{v}_{\mathrm{c}}=10^{-3} \mathrm{~m} / \mathrm{s}$ for carbon black and silica filled S-SBR 5025, respectively. Compared to filled S-SBR 5025 composites, the slightly higher level of adhesion for filled S-SBR 2525 systems is attributed to the real area of contact found to be higher at a given sliding velocity. Numerical parameters of hysteresis and adhesion friction simulations are summarized and discussed in Chapter 6.
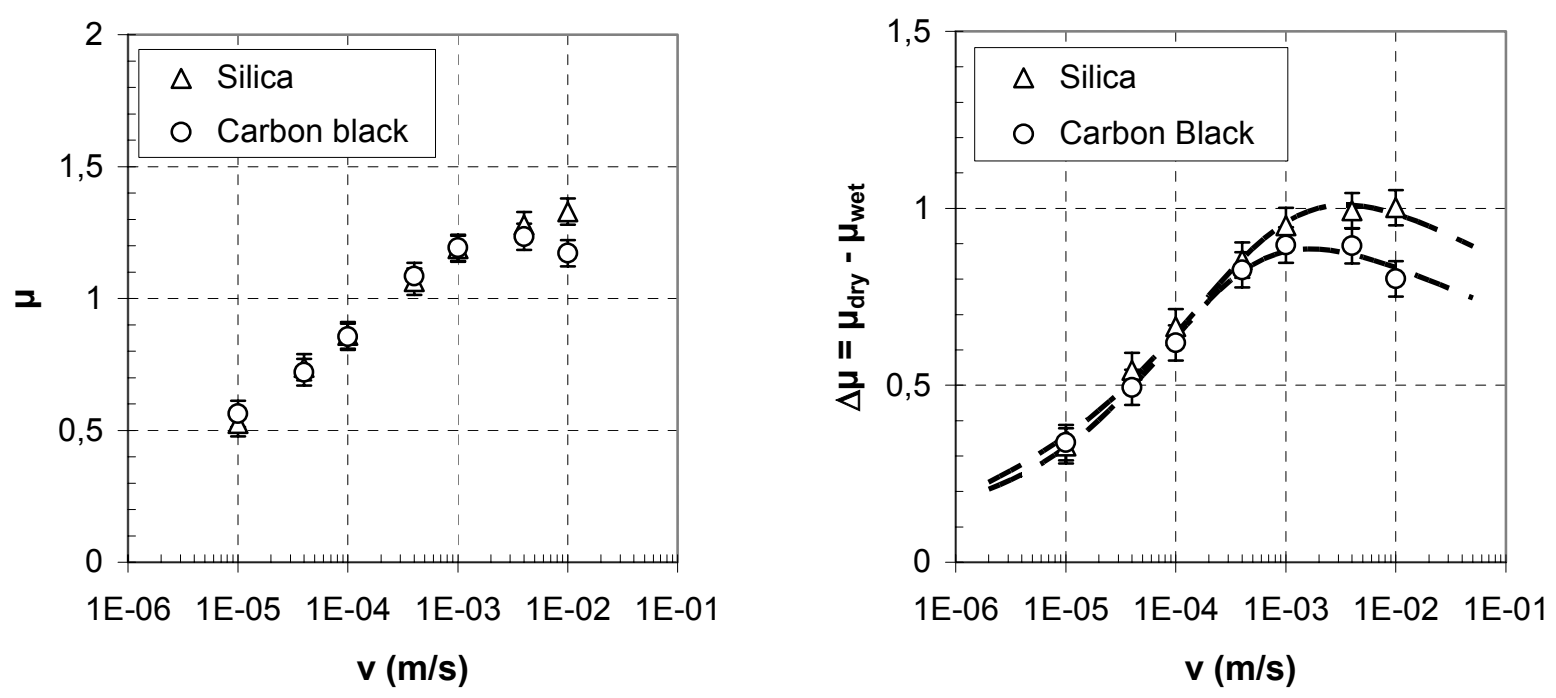

Figure 5.46: Dry friction results (left) and experimental difference dry/wet (right) with simulated adhesion component (dashed line) for silica and carbon black filled S-SBR 2525 on asphalt. Load $\sigma_{\circ}=12.3 \mathrm{kPa}$ 


\subsection{Correlations with friction measurements - II}

Previous experiments carried out on the modified Zwick testing equipment allows a characterisation of the friction coefficient up to a maximal sliding velocity $v=1 \mathrm{~cm} / \mathrm{s}$ and at moderate load $\sigma_{o}=12.3 \mathrm{kPa}$. In order to extent the range of measurements, a modified biaxial MTS test rig was used, allowing a maximal sliding velocity $v=40 \mathrm{~cm} / \mathrm{s}$ with the possibility to simulate typical loads encountered in tyre applications, namely $\sigma_{\circ}=250 \mathrm{kPa}$. Beside the advantages due to a characterisation of the friction process under realistic conditions, the combination of increased sliding velocity and load leads to the occurrence of temperature effects at least above $v=1 \mathrm{~cm} / \mathrm{s}$. However, due to a limited stroke of the horizontal MTS cylinder (max. $60 \mathrm{~mm}$ ), contact times decrease with increasing sliding velocity and should therefore limit the temperature increase within the contact area.

Figure 5.47 shows friction results for carbon black and silica filled SBR composites obtained on the same asphalt surface used for previous experimental investigations at moderate load $\sigma_{o}=12.3 \mathrm{kPa}$. The simulated hysteresis friction is shown and correlated with wet friction results under the assumption that rubber temperature keeps constant over the whole range of measurements. It can be seen that the theoretical prediction fairly agrees with all experimental results, at least up to a sliding velocity $v=1 \mathrm{~cm} / \mathrm{s}$ above which temperature effects might occur. In particular, wet friction results carbon black filled low- $T_{g}$-composites, namely the E-SBR 1500 and the S-SBR 2525, are well described by the hysteresis friction over the whole range of sliding velocity, whereby the corresponding increase is found to be restricted at a low level. The wet friction feature is more pronounced for high- $\mathrm{T}_{\mathrm{g}}$ and silica filled elastomers as observed in the range of moderate loads.

The overestimation of wet friction can be partly attributed to the impact of temperature at the interface and the occurrence of high deformations in the rubber at the vicinity of surface asperities which are not sufficiently taken into account by the averaged strain amplitude applied during dynamic mechanical measurements $(\varepsilon=3.5 \%)$. Nevertheless, since low- $\mathrm{T}_{\mathrm{g}^{-}}$ composites can be described by the model, this implies that another effect should be considered, namely the velocity dependence of the pre-factor $\left\langle\delta>/\left\langle\mathrm{z}_{\mathrm{p}}\right\rangle\right.$ which scales the simulated hysteresis friction and was up to here assumed to be constant over the whole range of sliding velocity. A detailed discussion is proposed in Chapter 6 for further extension of the modelling regarding the dynamic indentation behaviour of elastomers. 

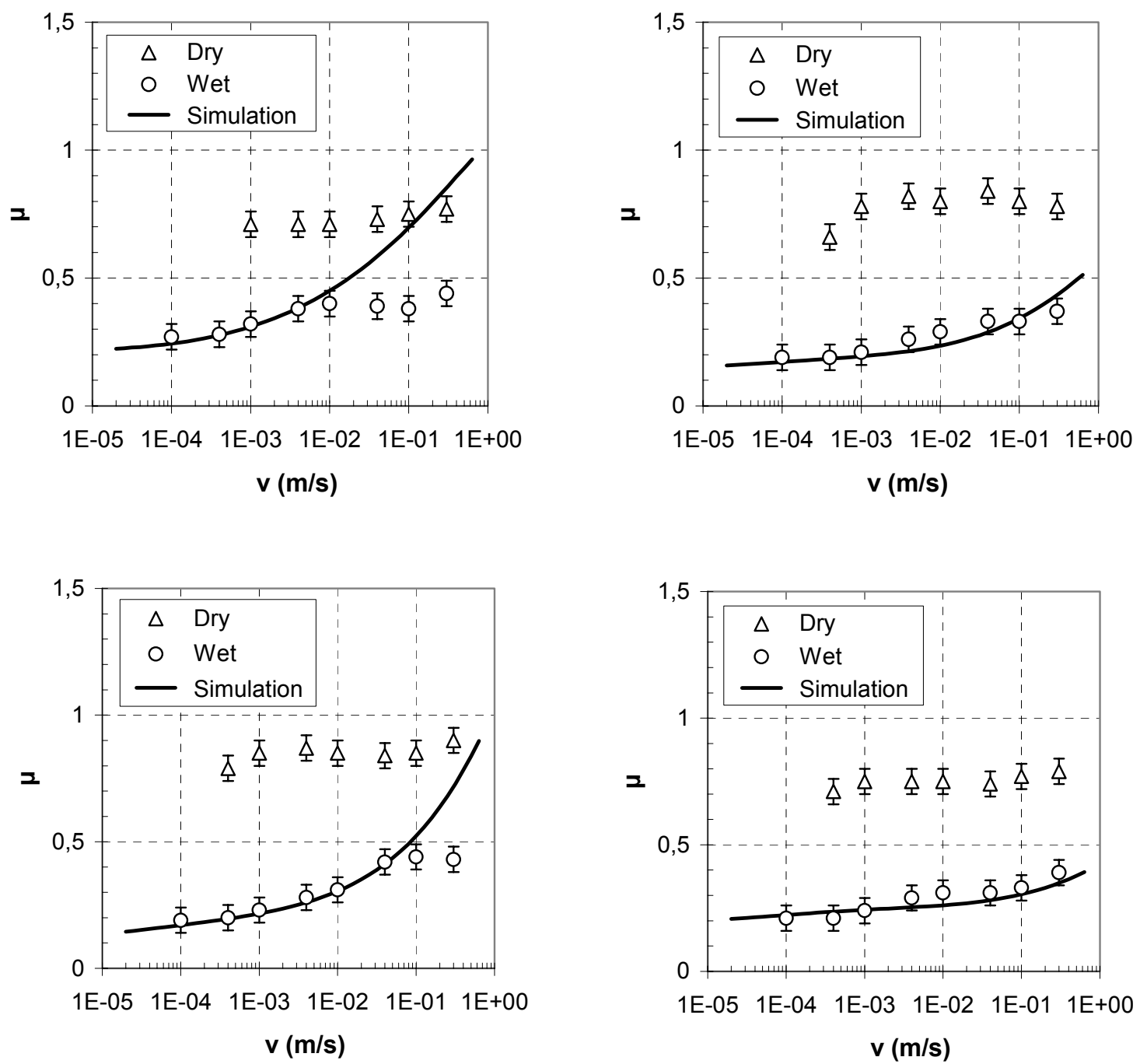

Figure 5.47: Friction results on asphalt and simulated hysteresis friction. Materials: S-SBR 5025 / N339 (top left), S-SBR 2525 / N339 (top right), S-SBR 2525 / Silica (bottom left) and E-SBR / N339 (bottom right). Load $\sigma_{o}=250 \mathrm{kPa}$

\subsection{Summary of results}

This chapter was dedicated to the applicability of hysteresis and adhesion friction concepts for the description of rubber stationary friction properties on rough surfaces. In particular, new insights were gained regarding the obtainment of surface and materials characteristics entering in the formulation of the proposed friction theory. The roughness analysis carried out by means of needle measurements highlights the relevance of a two-scaling-ranges approach for the description of texture and allows a separation between micro- and macrotexture regimes. Knowing that large length scales mostly contribute to hysteresis friction, this tool significantly improves the plausibility of the model. Furthermore, based on 
results obtained by means of dielectric and mechanical spectroscopy, a generalized master procedure was proposed for filled composites. Thereby, semi-empirical WLF-VFT functions describing the relaxation behaviour in the glass transition regime are not affected by increasing filler content and the overlapping of dynamic moduli in the low frequency region is ensured via vertical shift factors separately introduced for the storage and loss component. The temperature dependence of vertical shift factors is put in relationship with the variation of dynamic modulus above glass transition for filled composites and physically related to the activation energy of immobilised polymer nanobridges between adjacent filler particles. Pronounced differences are evidenced between silica and carbon black reinforced composites, whereby the presence of silica is associated with a dynamic softer behaviour above glass transition interpreted as an indication for favourable inter-locking properties. This is confirmed by model predictions where a broader contact interval and larger real contact area are found for silica filled composites compared to carbon black reinforced ones.

Simulations of hysteresis friction and contact parameters reveal the viscoelastic nature of rubber friction on rough surfaces. The introduction of a two-scaling-ranges approach is seen to reduce the contact interval and subsequently leads to a broader hysteresis friction maximum. The impact of the dynamic softening of filled elastomers above glass transition and vertical shift factors - on sliding process was investigated via a semi-empirical temperature law implemented in the model. The increase of contact temperature above $v \sim 1 \mathrm{~cm} / \mathrm{s}$ leads to a pronounced shift and reduction of the hysteresis friction maximum as well as a simultaneous increase of the contact interval and real contact area. Finally, the load dependence of the hysteresis friction coefficient was discussed on the basis of the G-W predictor $F_{1} / F_{3 / 2}$ found to be relevant at moderate sliding velocity up to the range of high loads. As a result, $\mu_{H}$ is found to decrease with increasing load on rough granite and asphalt.

Friction tests carried out at moderate load within the range of low sliding velocity under wet and dry conditions allow the determination of hysteresis and adhesion components. The use of a water detergent as lubricant is assumed to eliminate molecular interactions at the contact interface, so that wet friction results are identified with simulated hysteresis friction. The difference between wet and dry friction is interpreted in terms of adhesion and described by the real contact area combined with the interfacial shear strength. Results show the relevance of hysteresis friction concepts under wet conditions for filled composites on rough granite and asphalt. The modelling with a two-scaling-ranges approach leads to a further improvement in the description of wet friction, this effect being more pronounced for filled SSBR 5025 within the experimental range of sliding velocity. Concerning adhesion friction, improved inter-locking properties of silica filled composites are experimentally confirmed 
since the level of adhesion is found to be significantly higher than for carbon black filled composites. The position of the critical velocity depends on the glass transition temperature of the polymer matrix and the rough surface. Consequently, dry friction properties of elastomers on rough surfaces are driven by three mechanisms: the length scale dependent indentation process corresponding to hysteresis friction, the dynamic filling of profile cavities by rubber - real area of contact - and the kinetics of peeling effects related to the formation and breakage of contact patches. Thereby, the viscoelastic nature of rubber dry friction earlier demonstrated by Grosch is understood on a physical basis. 


\section{Discussion}

This chapter shows an analysis of the numerical parameters obtained trough correlations with wet and dry friction results, namely the pre-factor of the friction integral $b=\langle\delta\rangle \mid\left\langle z_{p}\right\rangle$ and the critical velocity $v_{c}$ of the interfacial shear strength $\tau_{s}$. Furthermore, a discussion is addressed regarding the frictional behaviour of unfilled elastomers. Following the theoretical frame of the model, a consistent extension is proposed on the basis of a velocity dependent pre-factor b suggested by additional friction results on filled and unfilled composites. The glass transition temperature of polymer matrix and the morphological state of filler network percolation threshold - appear to be determining for the dynamic indentation behaviour of elastomers during sliding friction on rough surfaces.

Values of the critical velocity $v_{c}$ are shown in Table 6.1 for filled and unfilled composites on rough granite and asphalt. Since hysteresis friction is found to continuously increase within the range of low sliding velocity, the critical velocity is located near the dry coefficient plateau value. A closer examination of the normalized interfacial shear strength $\tau_{\mathrm{s}} / \tau_{\mathrm{smax}}$ extrapolated over a broad range of sliding velocity reveals a systematic variation of the position of the critical velocity with respect to the glass transition [68]. The position of the adhesion peak is shifted towards higher sliding velocities with decreasing glass temperature. This viscoelastic feature of the adhesion component is consistent with the shift of the dry friction plateau obtained for filled elastomers under dry conditions [13][16]. Current investigations confirm the impact of glass transition temperature on the location of the critical velocity. As previously observed in Figure 5.40 and Figure 5.41, a sole variation of the S-SBR grade significantly shifts the position of the adhesion maximum on rough granite by about two decades, corresponding to the shift of frequency dependent dynamic modulus.

\begin{tabular}{cccccccc}
\hline & \multicolumn{3}{c}{ S-SBR 5025 } & \multicolumn{3}{c}{ S-SBR 2525 } & E-SBR \\
\hline & Unfilled & N339 & Silica & Unfilled & N339 & Silica & N339 \\
Rough granite & $2.10^{-5}$ & $2.10^{-6}$ & $6.10^{-6}$ & $7.10^{-5}$ & $4.10^{-4}$ & $8.10^{-4}$ & $3.10^{-4}$ \\
Asphalt & $4.10^{-5}$ & $3.10^{-5}$ & $6.10^{-5}$ & $3.10^{-4}$ & $7.10^{-4}$ & $10^{-3}$ & $10^{-4}$ \\
\hline
\end{tabular}

Table 6.1: Values of the critical velocity $v_{c}$ (in $\mathrm{m} / \mathrm{s}$ ) for filled and unfilled SBR grades on rough granite and asphalt 
Beside the influence of polymer matrix on the location of the adhesion peak, the filler system is found to control the level of adhesion via the real area of contact above critical velocity. Thereby, the dynamic mechanical softening above glass transition appears to be a key-factor for the ability of rubber to fill cavity of rough profile at various length scales. Accordingly, silica filled S-SBR composites exhibit favourable inter-locking properties in comparison with carbon black reinforced systems, as indicated by the evaluation of activation energy and the simulations of the real area of contact in Chapter 5 . The experimental procedure for the estimation of adhesion on rough surfaces confirms theoretical predictions and allows a deeper understanding of the mechanisms contributing to rubber friction on a physical basis. Finally, the impact of surface roughness is also indicated by a systematic shift of the values of critical velocity for asphalt. Since the velocity dependence of the interfacial shear strength is assumed to arise from the kinetics of peeling effects located at small length scales, the determining factor for the position of $\mathrm{v}_{\mathrm{c}}$ seems to be the fractal dimension of microtexture.

Next, values of the pre-factor $b=\langle\delta\rangle /\left\langle z_{p}\right\rangle$ are summarized in Table 6.2. Indeed, by referring to Equation (3.33), the formulation of $\mu_{H}$ is based on the assumption that the excitated layer $\langle\delta\rangle$ is related to the mean penetration depth $\left\langle z_{p}\right\rangle$ by a linear relationship. Since the latter is calculated via the Greenwood-Williamson functions, the level of hysteresis friction directly depends on the amplitude of the pre-factor $b=\langle\delta\rangle /\left\langle z_{p}\right\rangle$. Photogrammetry measurements showed that this condition is fulfilled under static conditions. The b-values were obtained by correlating wet friction results obtained in Chapter 5 with simulations of hysteresis friction modelled with a two-scaling-ranges approach. Thereby, indications of the deformed volume of elastomers during sliding friction can be gained. Accordingly, unfilled compounds exhibit significantly higher b-values compared to filled composites on both surfaces, suggesting that a larger amount of material is involved in the indentation process at a given normal force.

\begin{tabular}{cccccccc}
\hline & \multicolumn{3}{c}{ S-SBR 5025 } & \multicolumn{3}{c}{ S-SBR 2525 } & E-SBR \\
\hline \multirow{3}{*}{ Granite } & Unfilled & N339 & Silica & Unfilled & N339 & Silica & N339 \\
Asphalt & 20 & 7 & 11.2 & 34 & 10.6 & 11 & 9.2 \\
& 16 & 1.1 & 2 & 26 & 1.5 & 2.6 & 1.4 \\
\hline
\end{tabular}

Table 6.2: $\quad$ Values of the pre-factor $b=\langle\delta\rangle \mid\left\langle z_{p}\right\rangle$ obtained from simulated hysteresis friction with a two-scaling-regimes approach. Load $\sigma_{0}=12.3 \mathrm{kPa}$

Silica filled composites show larger values compared to carbon black filled compounds on both surfaces. Combined with the results obtained for unfilled compounds, the parameter $b$ 
seems to describe the material ability to deform during dynamic sliding contact since a similar trend is observed for the real area of contact. The impact of surface roughness on the deformed layer $\langle\delta>$ is significant and the pre-factor is strongly reduced on asphalt. Contrary to adhesion friction for which small length scales are crucial regarding the level of the real contact area and the kinetics of peeling effects, one expects the indentation behaviour to be controlled by the largest length scales of rough profile, namely the macrotexture and both cut-off lengths.

Results of b-values are further discussed on the basis of friction results obtained for unfilled S-SBR composites on rough granite and asphalt. Unfilled compounds display particular features regarding dynamic properties and friction behaviour. First, due to the absence of filler particles, the dependence of the dynamic moduli on strain amplitude is not observed (Payne effect). Therefore, the consideration of high strain amplitudes as a way to improve correlations between simulations of hysteresis friction and wet friction results for filled composites is not expected to be effective for unfilled elastomers. Figure 6.1 shows wet friction results of unfilled S-SBR composites on rough granite. As expected, the increase of wet friction is more pronounced than for filled compounds. This reflects the dynamic behaviour of unfilled compounds, whereby the frequency dependent loss factor tan $\delta$ exhibits a pronounced peak.
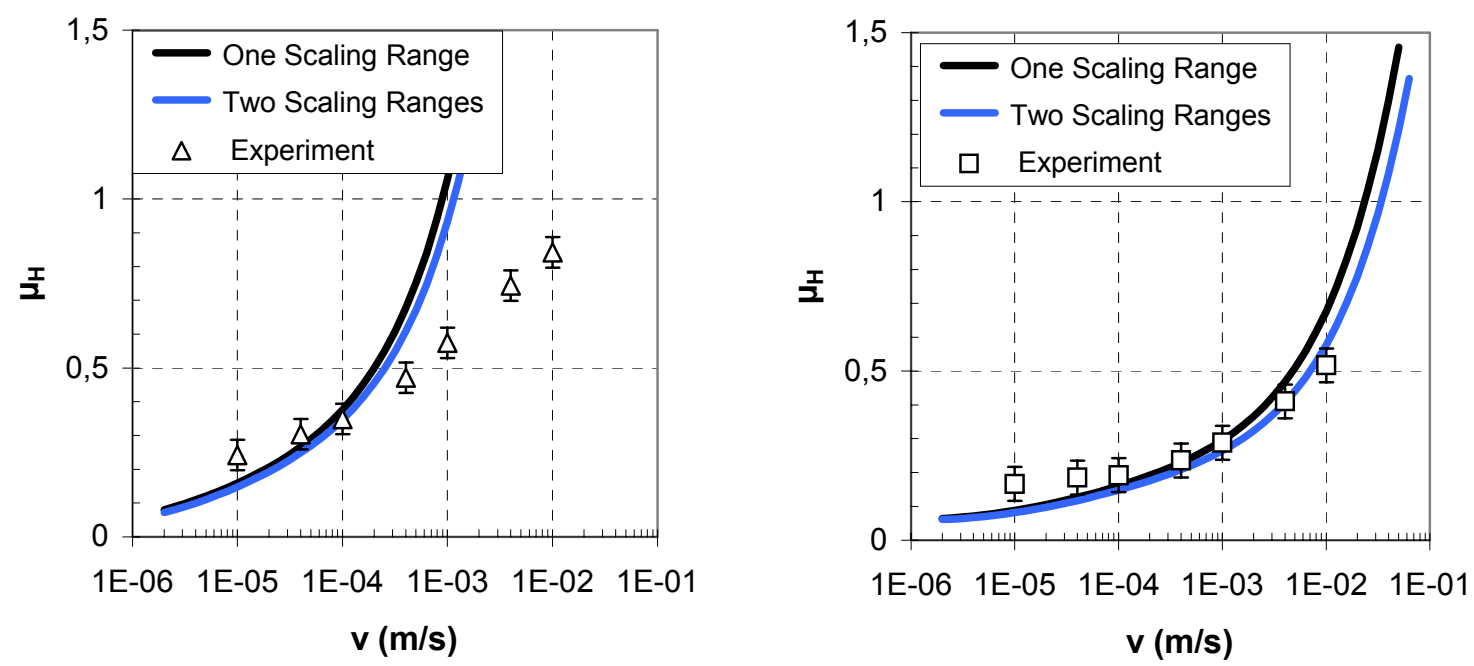

Figure 6.1: Wet friction results and hysteresis friction simulations for unfilled S-SBR 5025 (left) and S-SBR 2525 (right) on rough granite. Load $\sigma_{0}=12.3 \mathrm{kPa}$

Simulations of hysteresis friction are slightly improved with a two-scaling-ranges approach. For the unfilled S-SBR 5025 elastomer, a poor agreement is found between theory and experiment and the predicted friction largely overestimates experimental values above $\mathrm{v} \sim 1 \mathrm{~mm} / \mathrm{s}$. The S-SBR 2525 compound displays a more satisfying picture with a good 
description of wet friction data. A question arises from these results: which effect is not taken into account by the hysteresis friction model? Based on previous considerations, the temperature can not be responsible for the discrepancy of results, this would indeed imply a contact temperature $\mathrm{T} \sim 100^{\circ} \mathrm{C}$ at a sliding velocity $\mathrm{v}=1 \mathrm{~cm} / \mathrm{s}$.

Three possible interpretations can be considered regarding limitations of the current modelling of hysteresis friction. First, due to low hardness, unfilled compounds form larger contact area with rough substrates (Figure 5.23). As a consequence, the basic assumption $\mathrm{a}^{2}<<\mathrm{R}$, e.g. the contact radius is negligible compared to the asperity radius, might not be fulfilled for soft unfilled elastomers. Hence, simplifications made during the modelling of contact between elastomers and rough substrates may not be fulfilled and the contact mechanics of unfilled compound would require an extended formulation of the contact radii for this particular case. However, since increasing sliding velocity leads to a dynamic stiffening of elastomers, this effect is expected to occur within the rubbery region of the viscoelastic response, e.g. at very low sliding velocity. By referring to the left diagram of Figure 6.1 , the main problem is located close to the glass transition regime - at high sliding velocity - so that the assumptions formulated in the Hertz theory are not the limiting factor of the current multi-scale modelling of dynamic contact.

A second explanation accounts for the mode and amplitude of deformation during dynamic mechanical analysis. Indeed, measurements performed on the ARES Rheometer are based on a torsion of the rubber specimen up to a maximum experimental strain amplitude $\varepsilon=3.5 \%$. In order to reproduce realistic deformations undergone by elastomers during sliding process, an additional mechanical characterisation was carried out on dumbbell specimen with MTS testing equipment, thus allowing frequency and temperature dependent sweeps in a compressed state. Experimentally, the amplitude of sinusoidal excitation was gradually increased from $\varepsilon=1$ to $10 \%$ - peak to peak: from 2 to $20 \%$ - with a level of static precompression fixed at $\varepsilon_{\mathrm{c}}=10$ and $20 \%$. A master procedure was then applied on the dynamic moduli in the same way as presented in Chapter 5. Results of the dynamic loss factor are shown in Figure 6.2 for an unfilled S-SBR 5025 at a reference temperature $T=20^{\circ} \mathrm{C}$ with respect to the original data obtained on the Ares rheometer. It can be seen that the loss factor is mainly affected in the low and high frequency region. Due to the static precompression, dissipative effects appear to be promoted for frequency below $f=1 \mathrm{~Hz}$ compared to original measurements. A further increase of the amplitude of excitation has no significant influence of the loss factor and should therefore not affect the simulated hysteresis friction. The loss peak is also found to be larger in the high frequency region for precompressed samples with a slight shift of the maximum toward high frequencies. A similar 
trend is observed for the same material if the level of pre-compression is increased up to $\varepsilon_{c}=20 \%$ (see Appendix Figure 8.5).
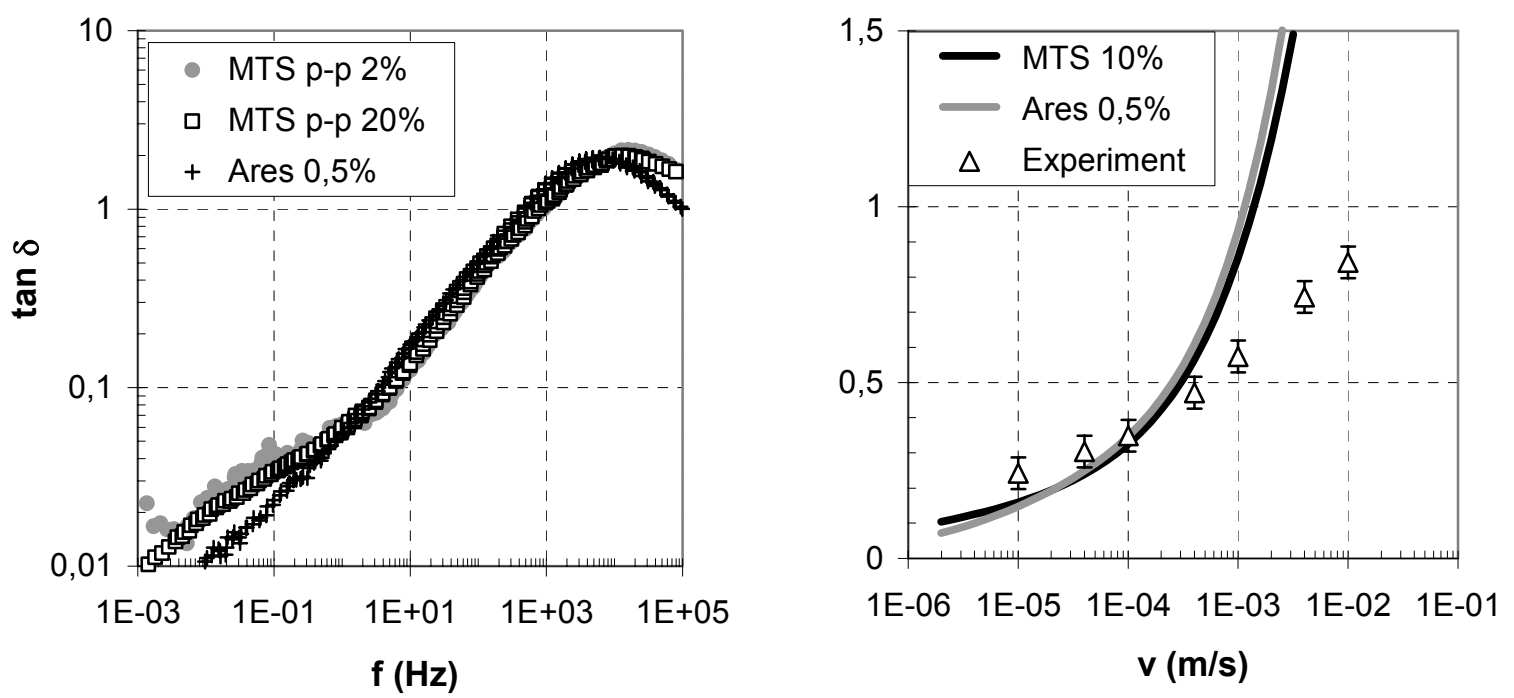

Figure 6.2: $\quad$ Master curves of loss factor for unfilled S-SBR 5025 on Ares and MTS with $10 \%$ static pre-compression and variable peak-to-peak amplitude at a reference temperature $T_{\text {ref }}=20^{\circ} \mathrm{C}$ (left). Wet friction results and influence of deformation mode on simulated hysteresis friction for unfilled S-SBR 5025 on rough granite (right). Load $\sigma_{0}=12.3 \mathrm{kPa}$

The corresponding hysteresis friction simulations are shown in Figure 6.2 on rough granite. As previously observed on dynamic mechanical data, the impact of deformation mode on the frequency dependent loss factor for unfilled composites is negligible over a wide range of frequency, and the resulting hysteresis friction feature is consequently not significantly modified. The higher level of mechanical losses found below $f=1 \mathrm{~Hz}$ appears to affect the simulation below sliding velocity $v \sim 10^{-5} \mathrm{~m} / \mathrm{s}$ where a cross-over of both simulated curves is observed. As a conclusion, the discrepancy between theoretical predictions and wet friction results can not be attributed to the mode of deformation and static constraints applied during dynamic mechanical analysis even if the MTS experimental set-up seems to be more relevant regarding the deformation of rubber during sliding process.

The third possibility consists in questioning the assumption that the pre-factor $b$ is independent of the sliding velocity. Beyond the apparent triviality of this hypothesis regarding friction data shown in Figure 6.2, further results indicate the relevance of a velocity dependent pre-factor on a physical basis. They are based on a similar study carried out on rough granite for silica filled S-SBR 2525 composites with a systematic variation of the filler content, thus extending current results obtained with 0 and $60 \mathrm{phr}$ silica and allowing the examination of the contribution of filler network on the friction behaviour. A satisfying 
correlation between simulations of hysteresis friction and wet friction results is realized over the range of filler content, namely from 0 to $80 \mathrm{phr}$. Accordingly, the resulting pre-factor $\mathrm{b}$ is shown in Figure 6.3 as a function of the filler content. It can be seen that the parameter $b$ exhibits a pronounced stepwise decrease around the percolation threshold of the elastomer located at $35 \mathrm{phr}$. The percolation threshold corresponds to a volume fraction of filler, where it can be assumed that a continuous interconnecting filler network exists in the polymer matrix. This can be evidenced by measuring electric properties of filled elastomers, whereby the electrical resistivity is relatively low above this volume fraction and the composite behaves essentially like an insulator below this volume fraction. Also, mechanical properties are significantly affected in the vicinity of the percolation threshold. Beyond the percolation threshold, no significant variation of the pre-factor is observed, which indicates a pronounced sensitivity of the indentation behaviour with respect to the filler network. The realization of a filler continuous path within the polymer matrix appears to be determining for the amount of material involved in the deformation process during sliding friction. Consequently, numerical observations made on the pre-factor basically reflect the microstructure of filled composites and the significant drop of the b-values observed in Table 6.2 appears to be a material specific feature.

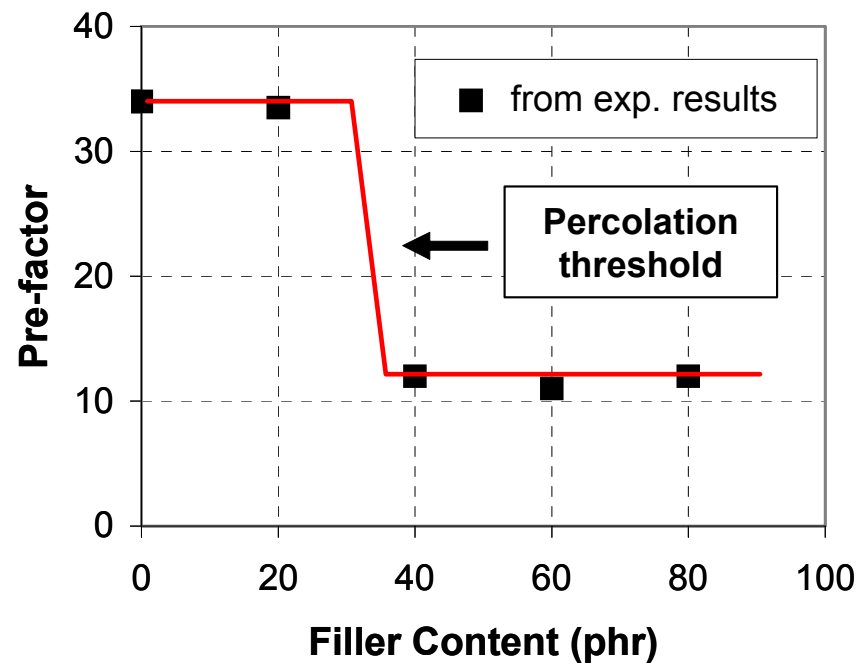

Figure 6.3: Values of pre-factor obtained from correlations with wet friction results as a function of silica content for S-SBR 2525 on rough granite. Load: $\sigma_{0}=12.3 \mathrm{kPa}$

Beside the major impact of filler networking on the pre-factor, another question arises from the results obtained for unfilled compounds, namely the poor agreement observed between theory and experiment for the S-SBR 5025 while the wet friction behaviour of the S-SBR 2525 could be fairly described by hysteresis friction. Since extensions of the Hertz theory and the consideration of a realistic mode of deformation during mechanical spectroscopy fail at 
solving this problem, the resulting assumption is that the parameter $b$ depends on the sliding velocity. Indeed, the indentation behaviour observed in Figure 6.3 indicates that the mechanical response of the elastomer controls the level of the pre-factor within a certain range of filler content - e.g. up to a given dynamic stiffness - followed by a region associated with a significant decrease of b-values and finally a plateau regime where a further addition of filler has no impact on the parameter $b$. Similar features are exhibited by the viscoelastic response of elastomers during sliding friction on rough surfaces: depending on the polymer type, rubber enters the glass transition regime above a given sliding velocity, going from a rubbery into a glassy state. Hence equivalent effects between the sliding velocity and the filler content regarding the increase of dynamic stiffness for elastomers. Following this argumentation, one could expect a similar dependence of the pre-factor $b$ on the sliding velocity as the one observed on the filler content Figure 6.4. Since the excitation frequency is directly related to the sliding velocity by Equation (2.18) and to the temperature via the semiempirical WLF-VFT equations, the horizontal axis of Figure 6.4 can be extended to these three quantities.

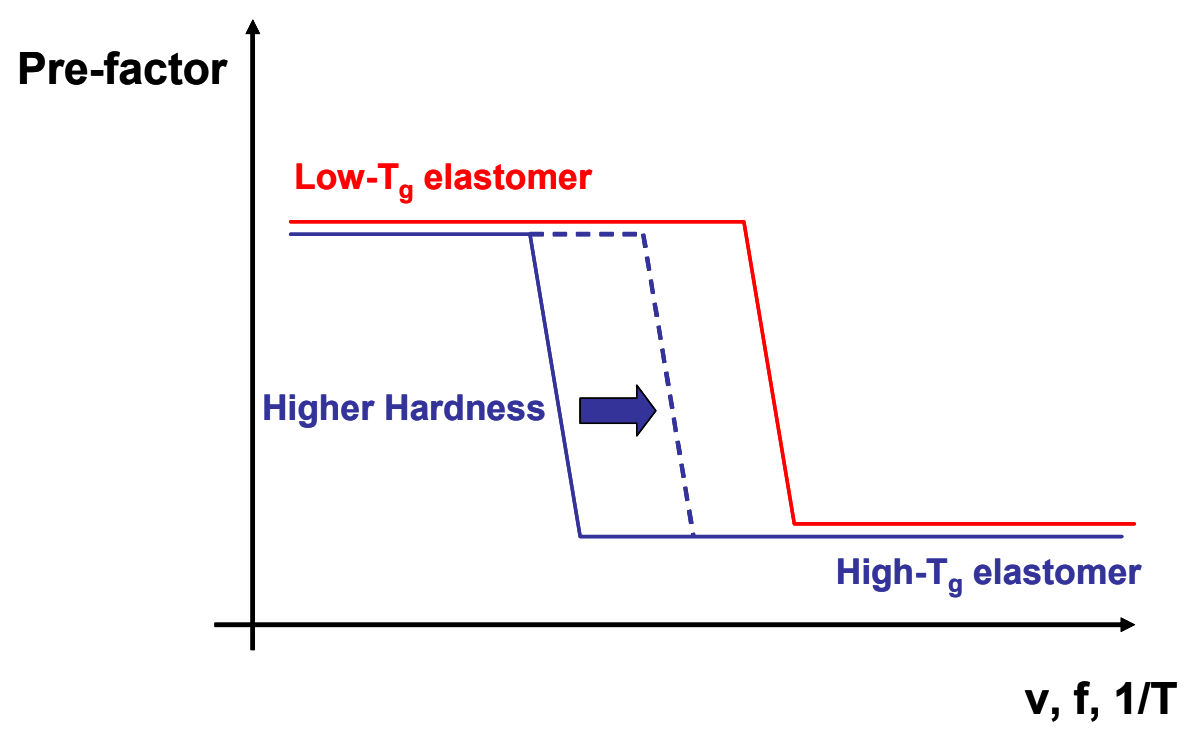

Figure 6.4: Schematic representation of the pre-factor as a function of sliding velocity, frequency and inverse temperature

As a result, the pre-factor can be assumed to be constant over the experimental range of sliding velocity $v=\left[10^{-5}-10^{-2} \mathrm{~m} / \mathrm{s}\right]$ for the unfilled S-SBR 2525 while a good description of wet friction results for the unfilled S-SBR 5025 would require to introduce a velocity dependent pre-factor above $v=10^{-4} \mathrm{~m} / \mathrm{s}$ (Figure 6.1). This suggests that the threshold velocity depends on the glass transition temperature of the elastomer and its position is shifted towards the region of high sliding velocity for low- $T_{g}$ composites. Also, since correlations between hysteresis friction simulations and wet friction results are satisfying for 
highly filled S-SBR 5025, the dynamic stiffness appears to give an additional contribution regarding the location of the threshold velocity, namely a shift towards the region of high sliding velocity for hard composites. Consequently, following this argumentation, two parameters seem to be relevant regarding velocity dependence of the pre-factor: the glass transition temperature of the polymer matrix and the dynamic stiffness characterised by the frequency dependent moduli. Since the information relative to the frequency and temperature dependent relaxation behaviour of elastomer is contained in the friction integral, the prefactor should be probably directly put in relationship with material moduli in the frame of a generalized modelling of hysteresis friction. 


\section{Conclusion and Outlook}

The stationary friction behaviour of elastomers on rough surfaces was investigated on the basis of a novel physically based modelling combined with experimental measurements carried out under specific conditions. The results give a deeper insight into physical mechanisms involved during sliding process regarding material properties and surface roughness.

\section{Physically motivated modelling of rubber friction}

Based on pioneering studies dealing with rubber friction, the friction coefficient was decomposed into hysteresis and adhesion friction. The hysteresis component arises from material losses associated with dynamic deformation of rubber by surface aspetities while adhesion is related to the amount of contact patches and the interfacial shear strength. $A$ decisive step for the modelling is achieved by considering the self-affine behaviour of rough surfaces which corresponds to a morphological invariance of their statistical properties under anisotropic dilations. Consequently, dynamic contact is distributed over many length scales and the generated hysteresis friction is integrated over a wide range of excitation frequency.

A general formulation of dynamic contact conditions was derived for soft materials against rigid rough substrates. It is based on an extended description of the elastic contact between rough surfaces proposed by Greenwood and Williamson. Following basic views from Hertz, the contact is approximated on the largest length scales, allowing a calculation of contact parameters like the mean penetration depth and load as a function of the so-called Greenwood-Williamson functions. Such quantities governed by macro-asperities require a fine description of the upper part of profile where contact actually occurs. This was achieved by introducing an analytical affine transformation which was compared to a numerical summit distribution evaluated within the range of the horizontal cut-off length. As a result, an affine parameter could be determined and further successfully implemented into the contact mechanics model.

Following refinements introduced for the formulation of dynamic contact, a special attention was dedicated to the description of surface roughness. In particular, the scaling behaviour of rough surfaces in the micrometer range is found to deviate at larger length scales which are 
determining for the level of hysteresis friction. As a result a second scaling range was introduced for length scales larger than $\lambda \sim 200 \mu \mathrm{m}$ associated with a different fractal dimension. Consequently, the hysteresis friction integral is decomposed into two distinct terms interpreted as the contributions of both micro- and macrotextures. The decoupling of texture regimes corroborates physical approaches used in the tyre industry for the prediction of wet grip behaviour. Since both texture regimes are associated with specific effects during dynamic sliding contact, the proposed approach minds the gap between road constructors and material developers regarding tyre grip performance on wet road tracks.

Beside hysteresis friction, a physical modelling of the adhesion friction on rough surfaces was proposed. It is based on the computed real area of contact combined with the velocity dependent interfacial shear strength. The amount of contact patches was analytically derived within the frame of the extended contact theory down to small length scales. The morphology of microtexture appears to be a relevant parameter for the occurrence of adhesion effects. Since the adhesion friction process is basically related to the formation and breakage of contact patches at the interface rubber/substrate, the velocity dependence of the interfacial shear strength is expected to describe the kinetics of corresponding peeling effects.

\section{Experimental results and simulations of rubber friction}

Roughness analysis carried out on rough granite and asphalt surfaces showed the relevance of a two-scaling-ranges approach for the description of texture. Compared to a classical treatment of roughness, the decomposition of texture leads to an increase of the horizontal cut-off length with significantly higher values of the fractal dimension associated with macrotexture. The values of the affine parameter are subsequently slightly modified by considering a second scaling regime. Also, from the equipment point of view, a complete characterisation of roughness requires high horizontal resolution combined with a sufficiently large measurement range. If one of these conditions is not fulfilled, the error on surface descriptors could mislead the prediction of hysteresis and adhesion friction.

Indentation tests performed by means of photogrammetry underlined the close relationship between the deformed layer of rubber and the penetration depth under static conditions. In particular, the occurrence of local high strains at the vicinity of the contact region suggests the necessity to consider higher strain amplitudes for dynamic moduli used in simulations

The measurement of dynamic properties of elastomers was achieved by means of relaxation spectroscopy methods. Through a combination of dielectric and dynamic mechanical 
spectroscopy, a generalized master procedure was proposed for filled composites. In particular, the addition of filler does not influence the main relaxation process located at the glass transition of the polymer matrix. The presence of filler particles induces non-linear effects observable on the dynamic behaviour due to complex interactions between the filler network and the polymer matrix. As a consequence, the classical WLF equation can not be applied in its simple form, but requires the introduction of vertical shift factors arising from superimposed Arrhenius-like or thermally activated processes. The corresponding activation energies were estimated for various filled composites and could be traced back to physical effects occurring on small length scales, namely the temperature dependence of immobilised polymer nanobridges between adjacent filler particles. The lower activation energies of silica filled S-SBRs were interpreted as favourable behaviour for improved interlocking properties between elastomers and rough substrates. Indeed, the calculation of the real area of contact shows significant larger values for silica filled composites over a broad range of sliding velocity.

Simulations of hysteresis friction and corresponding contact parameters were performed for various filled and unfilled SBR grades on rough granite and asphalt. The impact of filler could be identified and highlights the viscoelastic nature of hysteresis friction. Fundamental differences between silica and carbon black reinforced composites observed during dynamic mechanical analysis are predicted by the model for the S-SBR 5025 basis polymer with a pronounced friction peak for silica systems within the range of sliding velocities involved during ABS-Braking phases. This is due to favourable inter-locking properties through enhanced contact area.

The introduction of a second scaling range leads to a reduction of the hysteresis friction peak when entering the dynamic glass transition regime. This is due to a reduction of the contact interval combined with a higher fractal dimension of macrotexture. Also, the length scale delimitating micro- from macrotexture can be reached by the minimal length scale $\lambda_{\min }$, this situation being promoted by increasing sliding velocity and load. As a result the experimental range within which microtexture contributes to friction process can be fully determined for a given frictional pairing rubber / rough substrate and predictions agree with experimental observations made on standard grip testing equipments used by road constructors.

The occurrence of temperature effects at the contact interface with increasing sliding velocity was taken into account by an empirical temperature law motivated by former results obtained on rough surfaces as well as theoretical considerations. Consequently, the position and the amplitude of the hysteresis friction peak is strongly affected above $v=1 \mathrm{~cm} / \mathrm{s}$, whereby the 
impact of temperature dependent vertical shift factors is evidenced. Beside the reduced level of hysteresis friction, the real area of contact is found to increase at higher sliding velocities indicating that adhesion mostly contributes to dry friction under service conditions. The load dependence of hysteresis friction is considered via the Greenwood-Williamson ratio $F_{1} / F_{3 / 2}$. An equivalency is found between sliding velocity and load so that a single surface feature can be constructed for the whole range of experimental conditions.

Friction tests were carried out at moderate loads and low sliding velocities in order to prevent the increase of temperature in the contact area and also wear effects. Moreover, the use of a water detergent as lubricant is assumed to eliminate the adhesion component under wet conditions, allowing the identification of wet friction results with simulated hysteresis friction. The difference between wet and dry friction is interpreted in terms of adhesion and described by the real contact area combined with the interfacial shear strength. Results showed the relevance of hysteresis friction concepts under wet conditions for filled composites on rough granite and asphalt. In particular, improved correlations are achieved by using dynamic data measured at a strain amplitude $\varepsilon=3.0 \%$ in the simulation procedure. In addition the introduction of a two-scaling-ranges approach leads to a further improvement in the description of wet friction, this effect being more pronounced for filled S-SBR 5025 within the experimental range of sliding velocity.

A close relationship is found between the simulated real area of contact and the adhesion component for filled S-SBR 5025 composites. Improved inter-locking properties of silica filled composites are experimentally confirmed since the level of adhesion is found to be significantly higher than for carbon black filled composites. Thereby, the dynamic softening of filled elastomers above glass transition appears to be crucial during the formation of contact patches down to small length scales during sliding process. The position of the critical velocity is found to systematically vary with the glass transition of the polymer matrix, indicating the viscoelastic nature of adhesion friction and the role played by chain mobility during peeling processes distributed within the contact area. By extending hysteresis and adhesion friction correlations up to the range of high sliding velocity, a saturation of the dry friction coefficient is obtained over many decades. This corroborates pioneering results of Grosch where dry friction was seen to reach a saturation regime with increasing filler content. Through this observation, the relevance of scaling concepts for the modelling of hysteresis and adhesion friction of elastomers on rough surfaces is demonstrated. 


\section{Outlook}

Following the discussion proposed for the pre-factor of the friction integral, investigations on the dynamic indentation behaviour appear to be a promising route for an extended modelling of hysteresis friction. An exhaustive interpretation of friction results for filled and unfilled elastomers at low sliding velocities led to the conclusion that the pre-factor is likely to vary with sliding velocity. In particular, the transition observed on filled S-SBR 2525 around the percolation threshold indicates a strong influence of the material structural state on the dynamic indentation behaviour. A qualitative schematic variation of the pre-factor based on hardness and glass transition temperature has been proposed whose relevance has to be experimentally verified.

Even if the increase of contact temperature at high sliding velocities has been implemented in the model through an empirical temperature law, an analytical formulation of interfacial heat transfer mechanisms based on the current multi-scale description of dynamic contact still remains a challenge. Based on the frictional power generated during sliding friction, the increase of material temperature within the thickness can be calculated with basic approaches valid under static conditions. However, rough surfaces induce convection processes with a heat transfer from the rubber into the interfacial fluid - be it air or liquid which results in a complex mathematical formalism for the calculation of the temperature field. Hence the necessity to introduce a self-affine based formulation for the description of flash temperatures occurring locally on the rubber surface within a short amount of time after dynamic contact.

Dynamic and friction properties have been measured for model compositions. A next step would be to carry out a similar study on blend systems composed by a SBR basis polymer mixed with a low- $T_{g}$ elastomer typically entering in the formulation of tread compounds (NR, BR). New questions regarding the obtainment of high frequency data will arise, namely, new requirements on master procedure and the role of phase compatibility during dynamic relaxation processes. Also, complementary friction investigations should give new insights into the identification of material key parameters for improved friction properties.

Finally, since roughness appears to be a question of the length scale, the applicability of the presented concepts for the modelling of hysteresis and adhesion friction has to be investigated for smooth surfaces. Thereby, an extension of contact mechanics is necessary as the rubber roughness can not be assumed to be negligible on smooth surfaces. Regarding the scaling properties of macroscopically smooth substrates, recent white light 
interferometry measurements carried out on a smooth steel surface evidenced two distinct scaling regimes located below the micrometer range (Figure 7.1). Further extensions of modelling should mind the gap regarding the modelling of stationary rubber friction on rough and smooth surfaces.

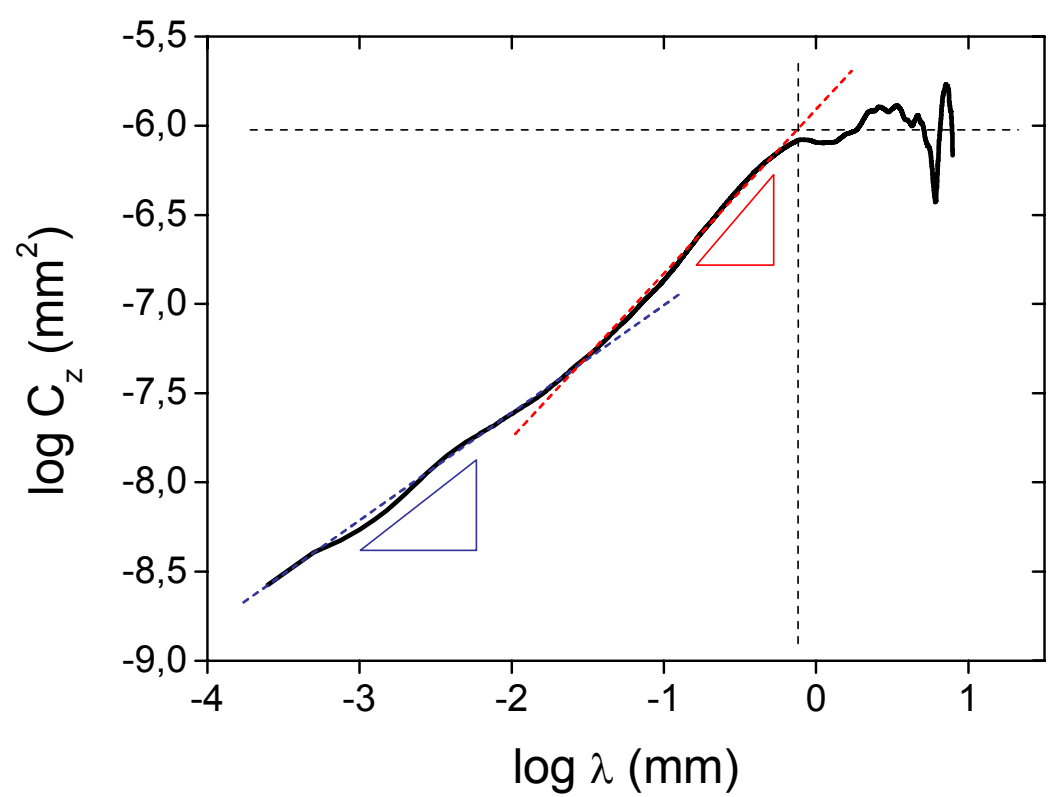

Figure 7.1: Height difference correlation function for a smooth steel surface characterised by means of white light interferometry measurements 


\section{Appendix}

\section{A. Generalized formulation of hysteresis friction with $\mathrm{n}$-scaling ranges}

By extending the scaling behaviour of the contact area $A_{c}(\lambda)$ expressed in Equations (3.25) and (3.38) to $n$ scaling regimes, one obtains:

$$
A_{c}(\lambda)=A_{c, 0} \prod_{i=1}^{n-1}\left(\frac{\lambda_{i+1}}{\lambda_{i}}\right)^{2-D_{i}}\left(\frac{\lambda}{\lambda_{n}}\right)^{2-D_{n}} \quad \text { for } \lambda<\lambda_{i}
$$

where $A_{c, 0}$ is the external contact area defined in Equation (3.39), $\lambda_{1}=\xi_{\| l}, \lambda_{n}=\sup \left\{\lambda_{i}\right.$ with $\left.\lambda_{\mathrm{i}}<\lambda_{\min }\right\}$ and $\lambda_{\mathrm{n}}=\lambda_{\min }$.

Similarly, the generalized form of the height difference correlation function $C_{z}(\lambda)$ with a nscaling-ranges approach is expressed as following:

$$
C_{Z}(\lambda)=\xi_{\perp} \prod_{i=1}^{n-1}\left(\frac{\lambda_{i+1}}{\lambda_{i}}\right)^{2 H_{i}}\left(\frac{\lambda}{\lambda_{n}}\right)^{2 H_{n}} \quad \text { for } \lambda<\lambda_{i}
$$

where $\lambda_{1}=\xi_{\|}, \lambda_{n}=\sup \left\{\lambda_{i}\right.$ with $\left.\lambda_{i}<\lambda_{\min }\right\}$ and $\lambda_{n}=\lambda_{\text {min }}$.

Accordingly, a generalized expression of the hysteresis friction coefficient is given by:

$$
\mu_{H} \equiv \frac{F_{H}}{F_{N}}=\frac{1}{2} \frac{<\delta>}{\sigma_{o} V} \sum_{i=1}^{n} \int_{\omega_{i}}^{\omega_{i+1}} \omega E^{\prime \prime}(\omega) \cdot S_{o, i}\left(\frac{\omega}{\omega_{i}}\right)^{-\beta_{i}} d \omega
$$

with $\omega_{1}=\omega_{\min }=2 \pi v / \lambda_{1}, \omega_{n}=\sup \left\{\omega_{i}, \omega_{i}<\omega_{\max }\right\}$ and $\omega_{n+1}=\omega_{\max } . S_{o, i}$ corresponds to the value of the power spectrum density at the intersection of the $i$-th and (i+1)-th scaling range. According to Equation (3.23) and knowing the exponent $\beta_{\mathrm{i}}$ associated with each scaling range, one obtains the following expression of $S_{o, i}$ : 


$$
S_{o, i}=\tilde{S}_{o, 1} \prod_{j=1}^{i-1}\left(\frac{\omega_{j}}{\omega_{j+1}}\right)^{\beta_{j}} \text { with } \quad \tilde{S}_{o, 1}=\frac{H_{1} \xi_{\perp}{ }^{2}}{2 \pi v \xi_{\|}}
$$

The summit density required for the calculation of contact parameters depends on the second and fourth momenta of roughness spectra found in a general case as:

$$
\begin{gathered}
m_{n}=\sum_{i=1}^{m} k_{i} \int_{f_{i}}^{f_{i+1}} f^{n}\left(\frac{f}{f_{i}}\right)^{-\beta_{i}} d f \\
\text { where } k_{i}=k_{1} \prod_{j=1}^{i-1}\left(\frac{\lambda_{j+1}}{\lambda_{j}}\right)^{\beta_{j}} \text { and } k_{1} \cong H_{1} \xi_{\perp}{ }^{2} \xi_{l \prime}
\end{gathered}
$$

As a result, the general form of the summit density $n_{s}$ is given by:

$$
n_{s}=\frac{m_{4}}{6 \pi \sqrt{3} m_{2}}=\frac{1}{6 \pi \sqrt{3}} \frac{\sum_{i=1}^{m} \prod_{k=1}^{i}\left(\frac{\lambda_{k}}{\lambda_{k+1}}\right)^{-\beta_{k}} \frac{\lambda_{i+1}{ }^{-5}}{5-\beta_{i}}}{\sum_{i=1}^{m} \prod_{k=1}^{i}\left(\frac{\lambda_{k}}{\lambda_{k+1}}\right)^{-\beta_{k}} \frac{\lambda_{i+1}{ }^{-3}}{3-\beta_{i}}} \text { with } \lambda_{m+1}=\lambda_{c}
$$

Following the analysis carried out for two scaling ranges, the formulation of the minimal length scale $\lambda_{\min }$ can be extended to $\mathrm{n}$ scaling ranges and leads to the following expression:

$$
\frac{\lambda_{\min }}{\xi_{\|}}=\left(\prod_{i=1}^{n-1}\left(\frac{\lambda_{i+1}}{\lambda_{i}}\right)^{8-3 D_{i}}\left(\frac{\lambda_{n}}{\xi_{\|}}\right)^{3 D_{n}-8} \frac{0.09 \pi s^{3 / 2} \xi_{\perp}\left|E^{*}\left(\lambda_{\min }\right)\right| F_{o}(d / \tilde{\sigma}) \tilde{n}_{s}}{\xi_{\| \mid}\left|E^{*}\left(\xi_{\| l}\right)\right| F_{3 / 2}\left(d / \tilde{\sigma}_{s}\right)}\right)^{\frac{1}{3 D_{n}-6}}
$$

$$
\text { with } \tilde{n}_{s}=6 \pi \sqrt{3} \lambda_{c}^{2} n_{s}
$$

Finally, a generalized formulation of the real area of contact associated with the contact interval $\left[\lambda_{\min }, \xi_{\| l}\right]$ for $\mathrm{n}$ scaling ranges follows Equation (3.46) with $\mathrm{n}_{\mathrm{s}}$ given by Equation (8.6). 


\section{B. Complementary dielectric results}

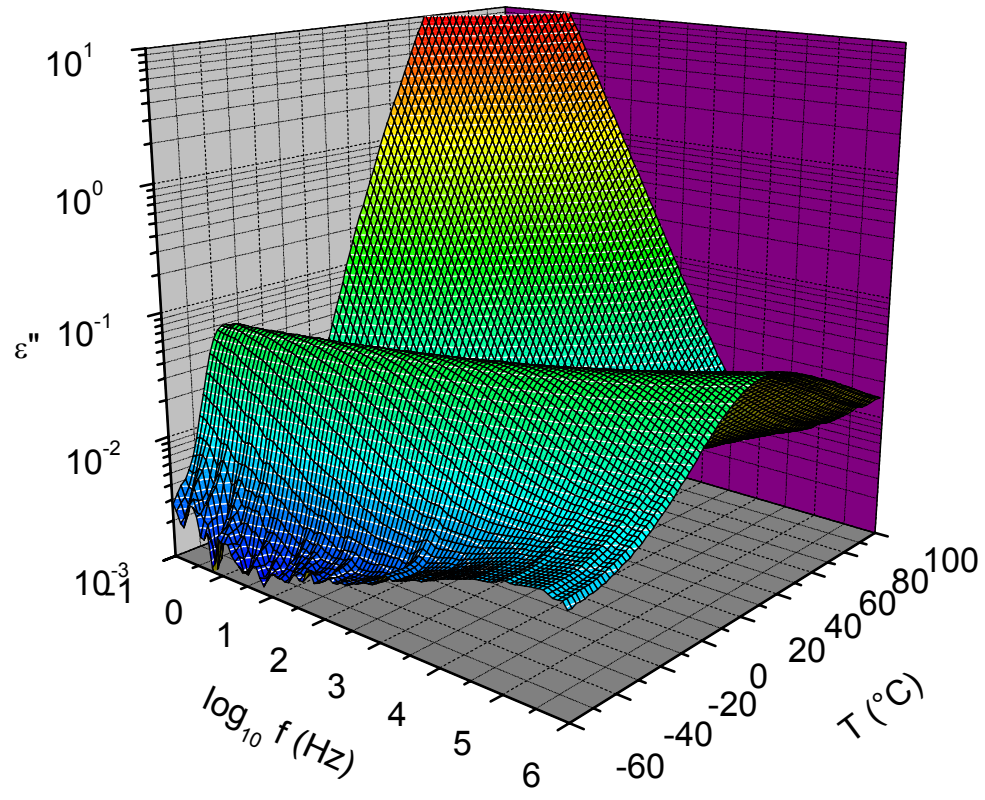

Figure 8.1: Measurement of the temperature and frequency dependent dielectric loss $\varepsilon "(\omega, T)$ for an unfilled S-SBR 2525
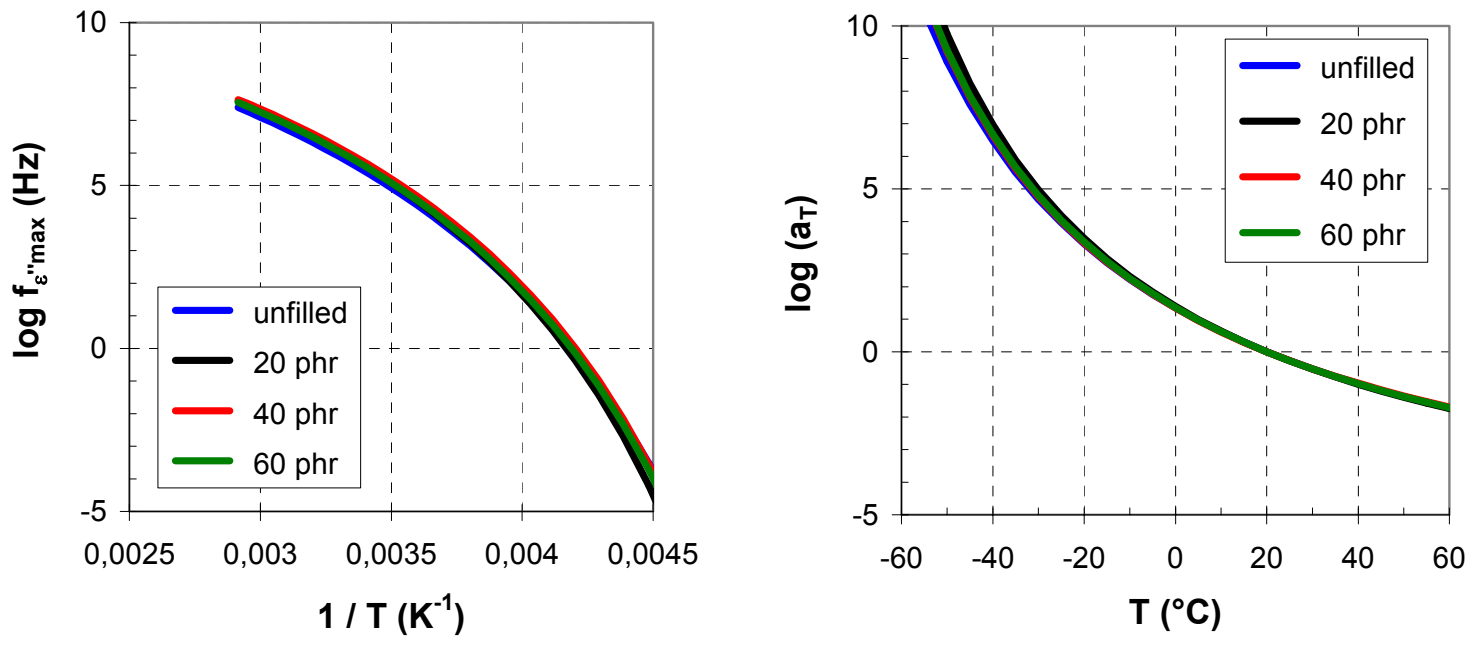

Figure 8.2: Activation Plots for silica filled S-SBR 2525 (left) and associated WLF functions (right) 


\section{Complementary dynamic mechanical results}

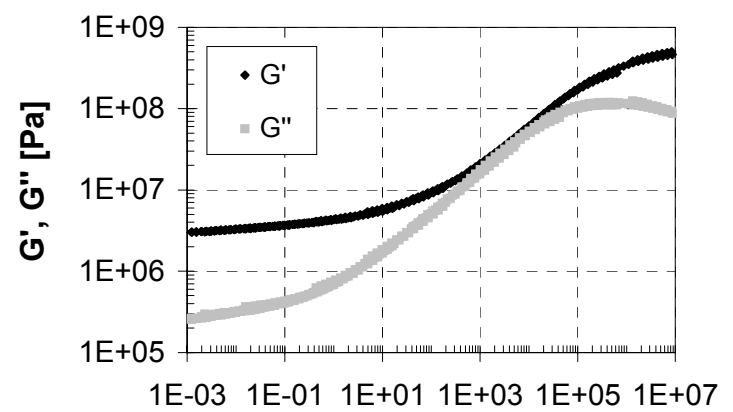

$\mathrm{f}[\mathrm{Hz}]$

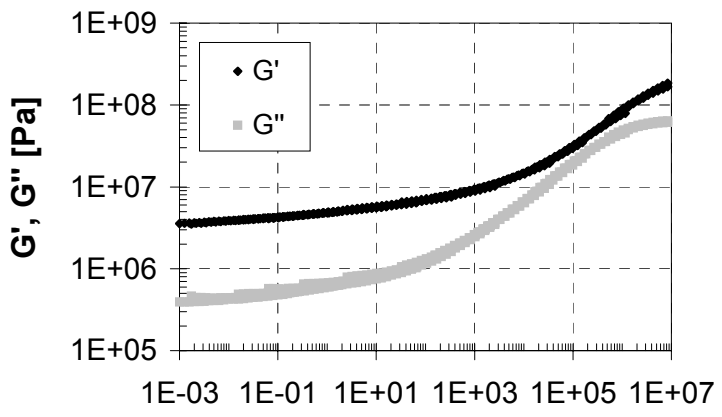

$f[\mathrm{~Hz}]$

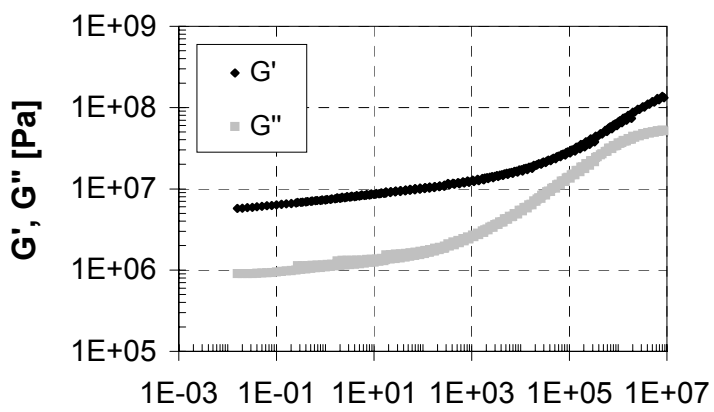

$f[\mathrm{~Hz}]$

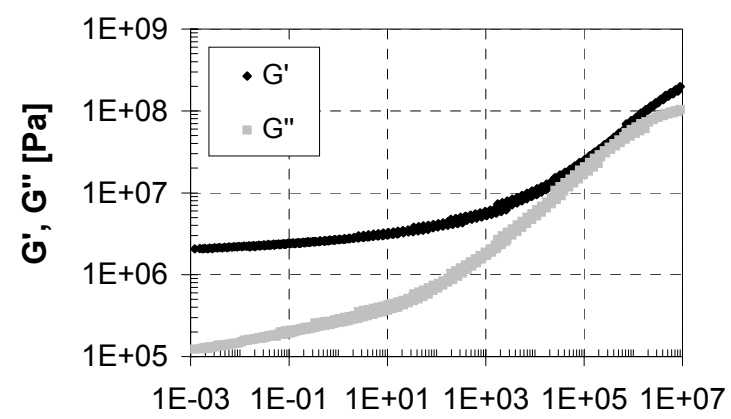

$f[\mathrm{~Hz}]$

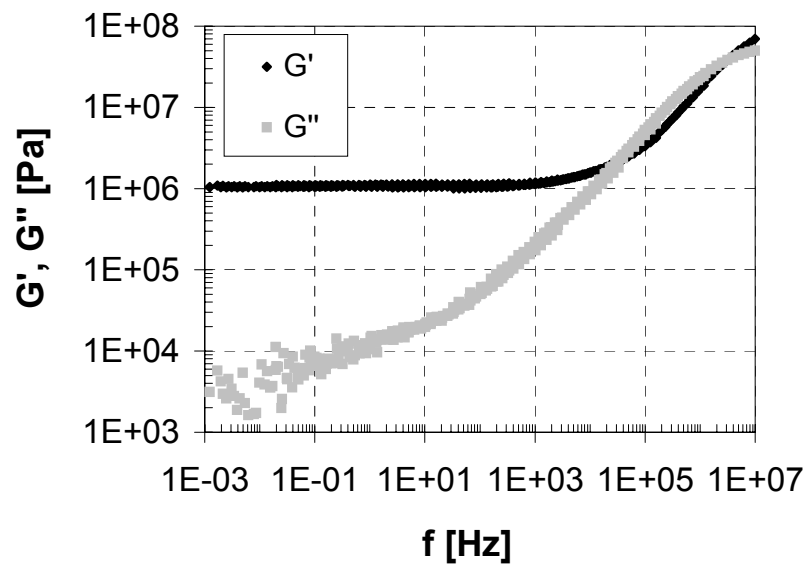

Figure 8.3: $\quad$ Master curves of dynamic moduli for filled SBRs at a reference temperature $\mathrm{T}_{\text {ref }}=20^{\circ} \mathrm{C}$ and strain amplitude $\varepsilon=0.5 \%$. Top left: S-SBR $5025 / 60 \mathrm{phr}$ Silica. Top right: E-SBR 1500 / 60 phr N339. Middle left: S-SBR 2525 / 60 phr N339. Middle right: S-SBR 2525 / 60 phr Silica. Bottom: S-SBR 2525 Unfilleda 

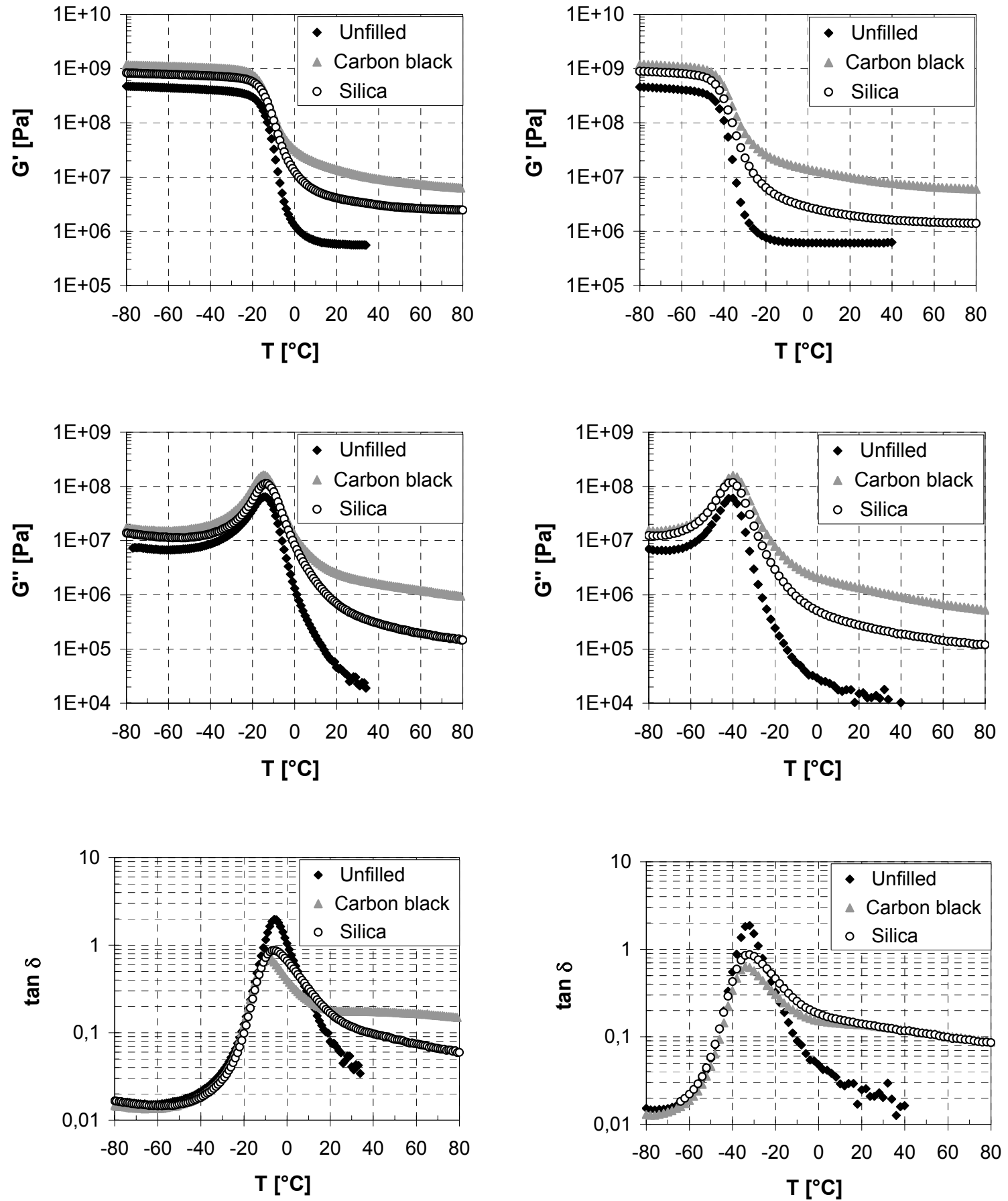

Figure 8.4: Temperature dependent dynamic storage modulus G' (top), loss modulus G" (middle) and loss factor $\tan \delta$ (bottom) of filled and unfilled elastomers with a S-SBR 5025 (left) and S-SBR 2525 (right) basis polymer. Filler content: 60 phr $(f=1 \mathrm{~Hz}, \varepsilon=0.5 \%)$ 


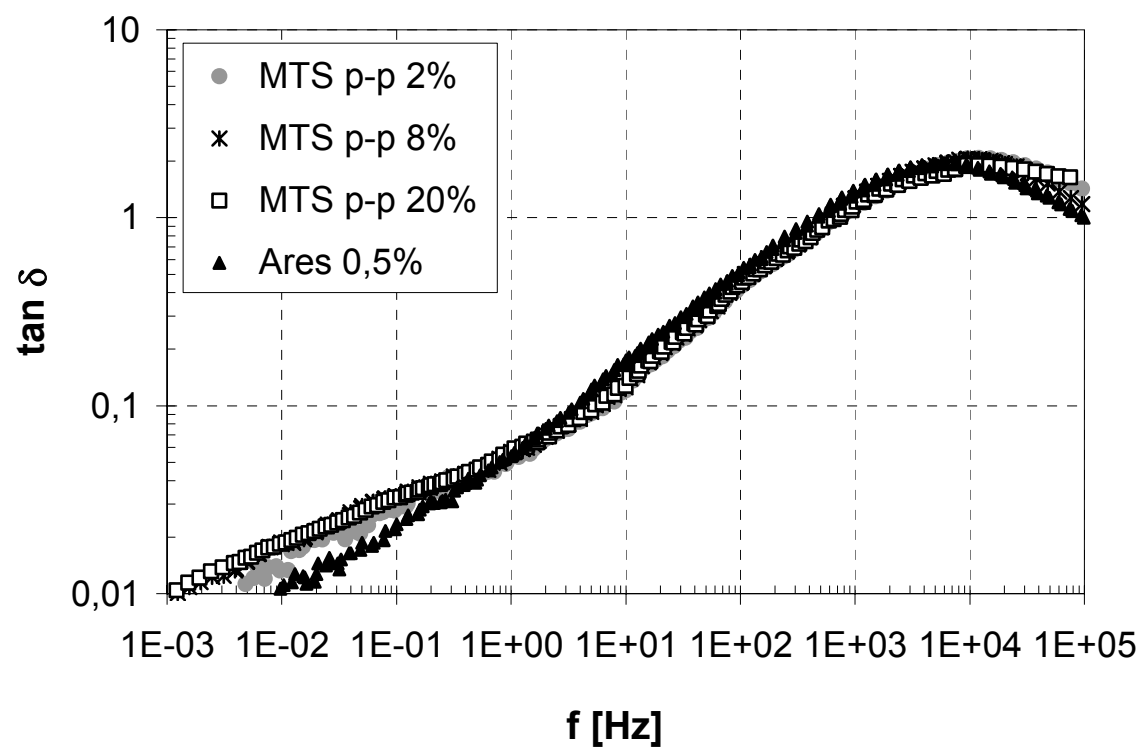

Figure 8.5: Master curves of unfilled S-SBR 5025 on Ares and MTS with 20\% compression and variable peak-to-peak amplitude. $T_{\text {ref }}=20^{\circ} \mathrm{C}$ 
D. Complementary simulations and correlations
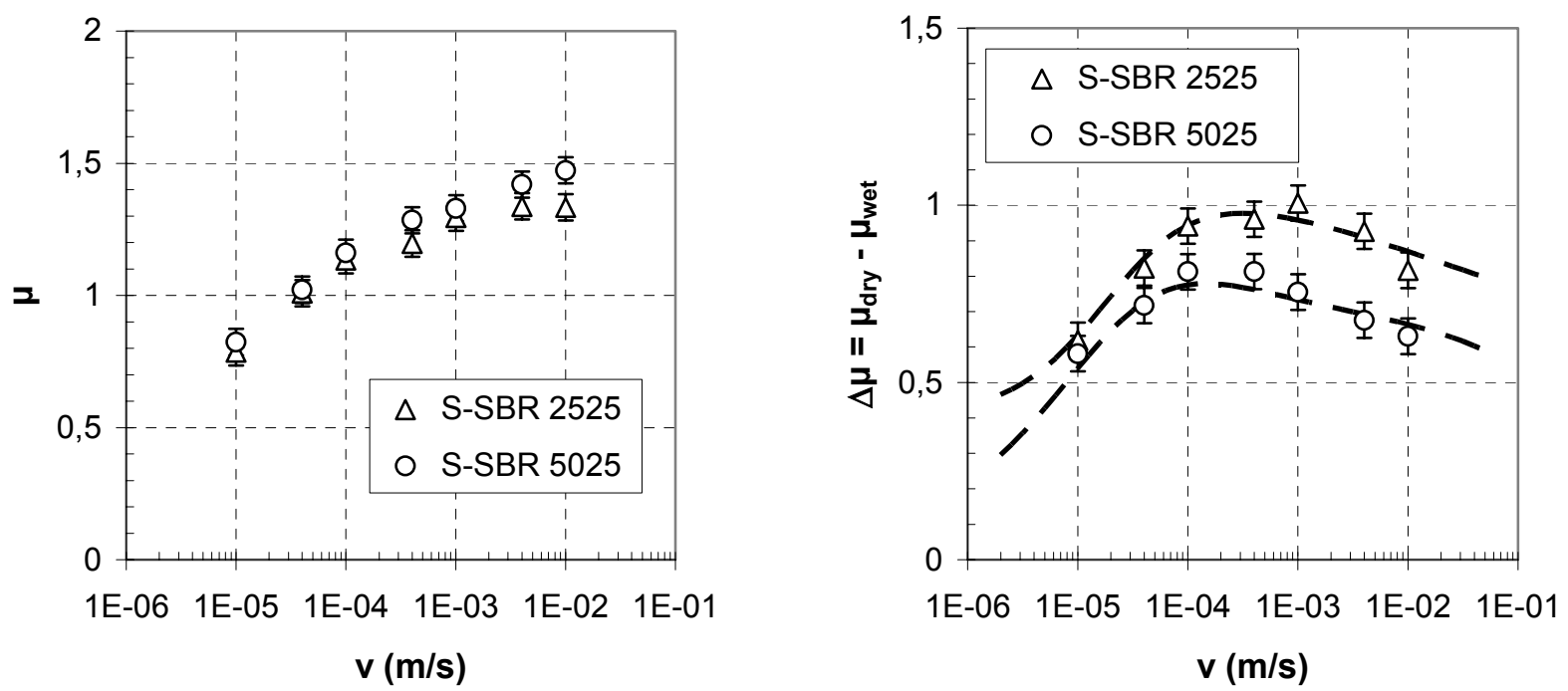

Figure 8.6: Dry friction results (left) and difference dry/wet (right) with simulated adhesion friction (dashed lines) for unfilled S-SBR 5025 and S-SBR 2525 on rough granite. Load $\sigma_{0}=12.3 \mathrm{kPa}$
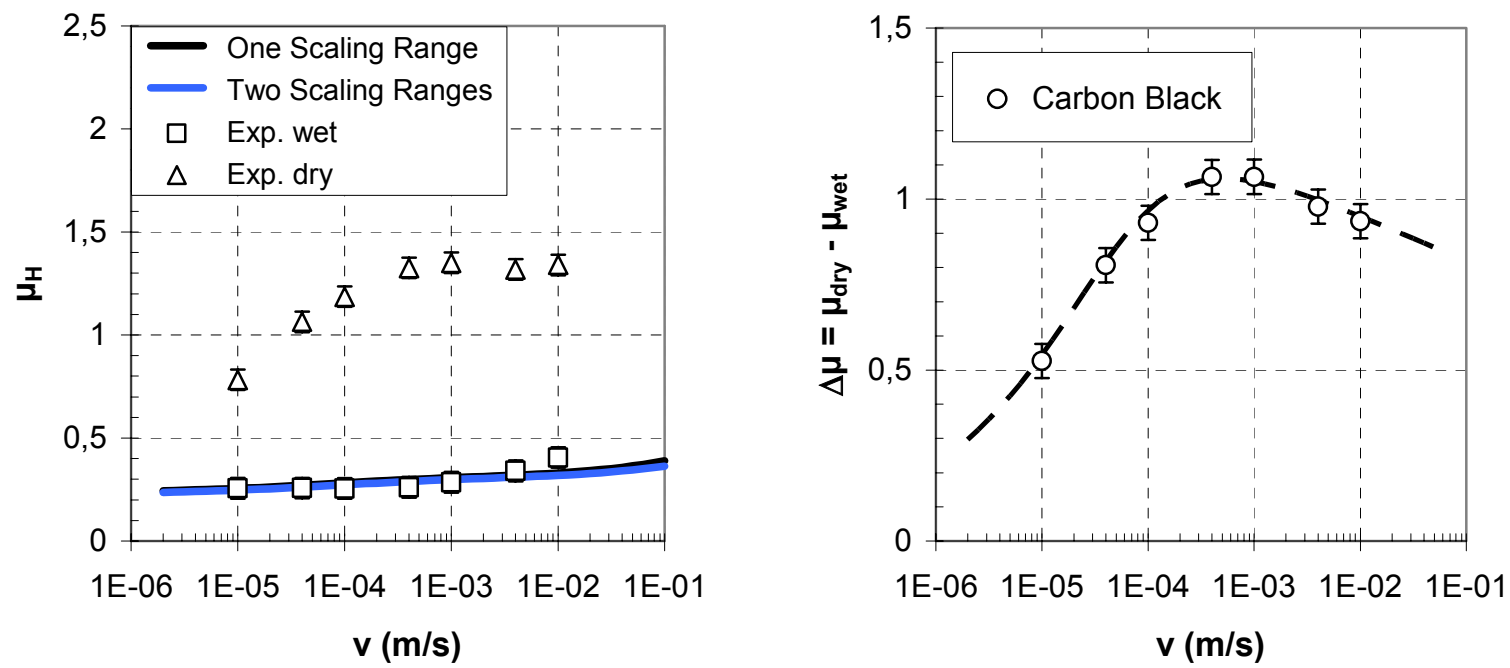

Figure 8.7: Wet and dry friction results for carbon black filled E-SBR 1500 on asphalt with corresponding simulated hysteresis friction (left) and experimental difference dry/wet (right) with simulated adhesion component (dashed line). Load $\sigma_{o}=12.3 \mathrm{kPa}$ 

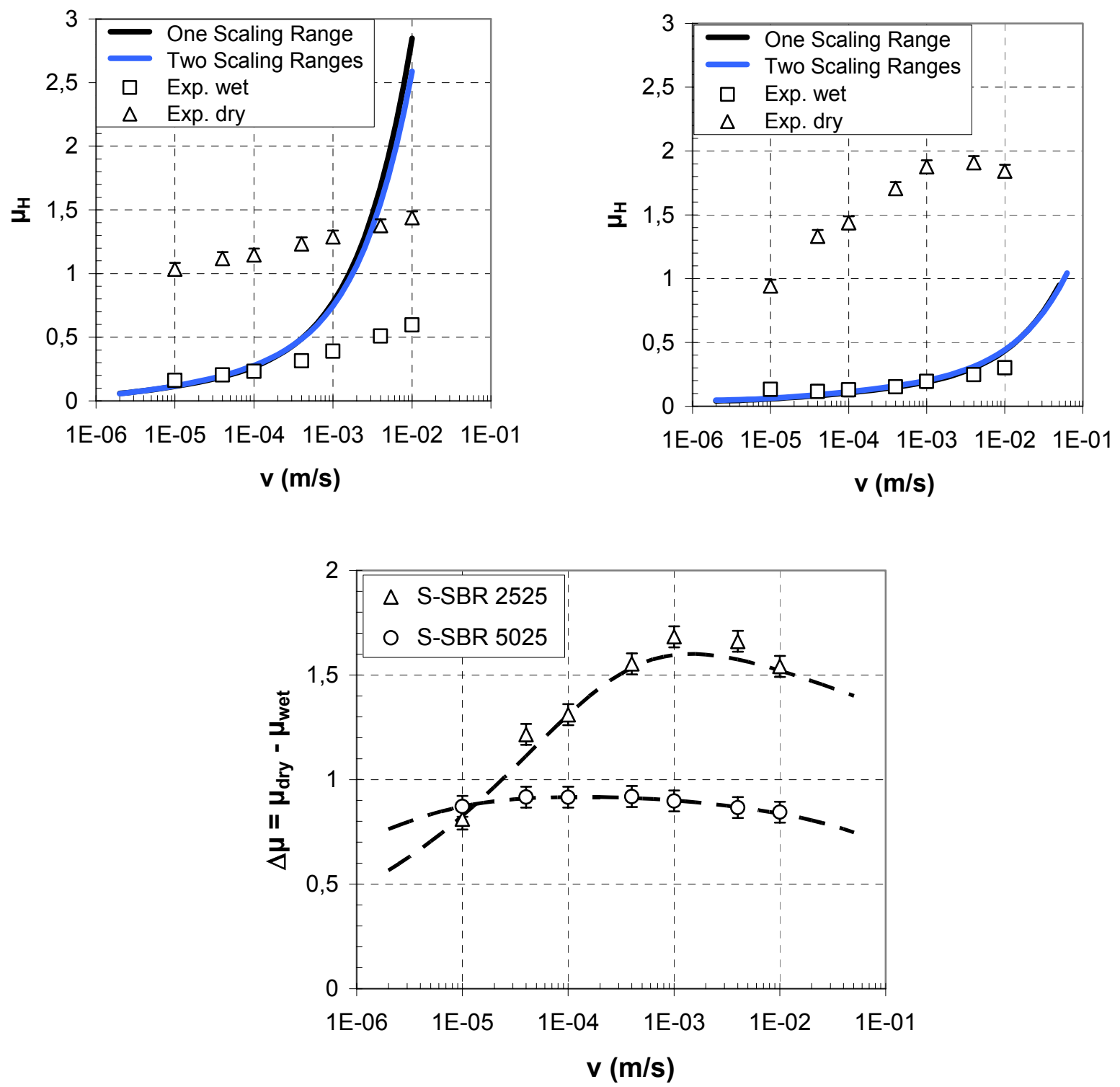

Figure 8.8: Wet and dry friction results for unfilled S-SBR 5025 (top left) and S-SBR 2525 (top right) on asphalt with corresponding simulated hysteresis friction and experimental difference dry/wet (bottom) with simulated adhesion component (dashed line). Load $\sigma_{o}=12.3 \mathrm{kPa}$ 


\section{E. List of publications and oral presentations}

[1] A. Le Gal and M. Klüppel, "Investigation and modelling of rubber sliding friction on rough surfaces", Journal of Physics D: Condensed Matter, 20, 015007 (2008)

[2] A. Le Gal, L. Guy, G. Orange, Y. Bomal and M. Klüppel, "Modelling of sliding Friction for carbon black and silica filled elastomers on road tracks", Wear, 264, 606 (2008)

[3] A. Le Gal and M. Klüppel, "Investigation and modelling of rubber adhesion friction on rough surfaces", Kautschuk Gummi Kunstst. 65, 308 (2006)

[4] A. Le Gal, X. Yang and M. Klüppel, "Evaluation of sliding friction and contact mechanics of elastomers based on dynamic mechanical analysis", J. Chem. Phys. 123, 014704 (2005)

[5] A. Le Gal, X. Yang and M. Klüppel, "Modelling of rubber friction: A quantitative description of the hysteresis and adhesion contribution", Constitutive Models for Rubber IV, 171 (2005)

[6] A. Le Gal, T. Meyer and M. Klüppel, "Modelling of Hysteresis and Adhesion Friction on rough surfaces", 7th Kautschuk-Herbst-Kolloquium, Hannover, November 8-11 (2006)

[7] A. Le Gal, T. Meyer and M. Klüppel, "Impact of road texture on rubber frictional properties", International Rubber Conference (IRC), Lyon, May 16-18 (2006)

[8] A. Le Gal, M. Klüppel, "Prediction of rubber friction properties based on dynamic mechanical analysis", MTS European Elastomer Testing User Society, Paris, March 16-17 (2006)

[9] A. Le Gal, M. Klüppel, "Tyre Materials, Safety Constraints and Performance", Energy efficient tyres, International Energy Agency, Paris, November 15-16 (2005)

[10] A. Le Gal, X. Yang and M. Klüppel, "Modelling of Rubber Friction: A quantitative description of the hysteresis and adhesion contribution", 4th European Conference on Constitutive Models for Rubber (ECCMR), Stockholm, June 27-29 (2005)

[11] A. Le Gal and M. Klüppel, "Hysteresis and adhesion friction on dry and wet tracks", Seminar Friction - Traction, Hannover, September 22. (2005)

[12] A. Le Gal, X. Yang and M. Klüppel, "Sliding Friction and Contact Mechanics of Elastomers on Rough Surfaces", 4th Contact Mechanics International Symposium (CMIS), Loccum, July 4-6 (2005)

[13] A. Le Gal, X. Yang and M. Klüppel, "Untersuchung und Modellierung der Elastomerreibung auf rauen Oberflächen", 286. Veranstaltung der Bezirksgruppe Niedersachsen der DKG, Hannover, April 5 (2005) 
[14] A. Le Gal and M. Klüppel, "Contact Mechanics on Rough Surfaces and Modelling of Rubber Sliding Friction", Symposium Rolling Contact Mechanics, GAMM Conference, Luxemburg, March 28-31 (2005)

[15] A. Le Gal and M. Klüppel, "Evaluation of hysteresis friction on dry and wet tracks", Seminar Safety - Wet Grip - Traction, Hannover, April 27. (2004)

[16] A. Le Gal, X. Yang, A. Müller and M. Klüppel, "From dynamic mechanical analysis to hysteresis and adhesion friction of elastomers on rough surfaces", Poster, 6th KautschukHerbst-Kolloquium, Hannover, November 10-13 (2004)

[17] M. Klüppel and A. Le Gal, "Hysteresis and adhesion friction on dry and wet tracks", Seminar Friction - Traction, Hannover, September 14. (2006)

[18] T. Meyer, A. Le Gal and M. Klüppel, "Mechanical modelling of friction and adhesion of elastomers at rough interfaces", European Conference on Contact Mechanics (ECCM), Lisbon, June 10-12 (2006) 


\section{Literature}

[1] D.F. Moore, The Friction and Lubrication of Elastomers, Pergamon Press, Oxford (1972) and the friction of pneumatic tyres, Elsevier scien. Publish. company (1975)

[2] A.N. Gent, Engineering with rubber, $2^{\text {nd }}$ Edition, Hanser, Cincinnati (2000)

[3] J.E. Mark, B. Erman, F.R. Eirich, Science and Technology of Rubber, $2^{\text {nd }}$ Edition, Academic Press, San Diego (1994)

[4] H. Krause, G. Poll, Mechanik der Festkörperreibung, Düsseldorf, VDI Verlag (1980)

[5] Société MICHELIN, Le pneu - l'adhérence, Société de Technologie Michelin (2000)

[6] A.R. Payne: "The dynamic properties of carbon black-loaded natural rubber vulcanisates, Part I", Rubber Chem. Technol. 36, 432 (1963)

[7] A.R. Payne: "The dynamic properties of carbon black-loaded natural rubber vulcanisates, Part II", Rubber Chem. Technol. 36, 444 (1963)

[8] M.L. Williams, R.F. Landel, J.D. Ferry: "The temperature dependence of relaxation mechanisms in amorphous polymers and other glass forming liquids", J. Am. Chem. Soc. 77, 3701 (1955)

[9] J.D. Ferry, Viscoelastic properties of polymers, $2^{\text {nd }}$ ed., Wiley, N.Y. (1970)

[10] H. Vogel, Phys. Zeit. 22, 645 (1921)

[11] G.S. Fulcher, Journal of the American Ceramic Society 8, 339 (1925)

[12] G. Tammann, W. Hesse, Z. Anorg. Allg. Chem. 156, 245 (1926)

[13] K.A. Grosch: "Relation between friction and viscoelastic properties of rubber", Proc. Roy. Soc. Lond. A 274, 21 (1963)

[14] K.A. Grosch: "The rolling resistance, wear and traction properties of tread compounds", Rubber Chem. Technol. 69, 495 (1996)

[15] K.A. Grosch: "Laborbestimmung der Abrieb- und Rutschfestigkeit von Laufflächenmischungen - Teil I: Rutschfestigkeit", Kautschuk Gummi Kunststoffe 49, 432 (1996)

[16] H. Rieger, Experimentelle und theoretische Untersuchungen zur Gummireibung, PhD Thesis, Technische Universität Munich, Germany (1968)

[17] H.W. Kummer: "Lubricated friction of rubber - Discussion", Rubber Chem. Technol. 41, 895 (1968)

[18] H.W. Kummer, W.E.Meyer: "Verbesserter kraftschluss zwischen Reifen und Fahrbahn - Ergebnisse einer neuen Reibungstheorie", ATZ 69/8, 245 (1967)

[19] A.N. Gent and J. Schultz: "Effect of wetting liquids on the strength of adhesion of viscoelastic solids", J. Adhesion 3, 281 (1972) 
[20] M. Barquins, A.D. Roberts: "Rubber friction variation with rate and temperature : some new observations", J. Phys. D 19, 547 (1986)

[21] M. Barquins: "Adherence, friction and wear of rubber-like materials", Wear 158, 87 (1992)

[22] D. Maugis and M. Barquins, J. Phys. D11, 1989 (1978)

[23] P.G. de Gennes, Langmuir 12, 4497 (1996)

[24] A.D. Roberts: "A guide to estimating the friction of rubber", Rubber Chem. Technol. 65, 673 (1992)

[25] A.D. Roberts, A.G. Thomas: "The adhesion and friction of smooth rubber surfaces", Wear 33, 45 (1975)

[26] A.D. Roberts: "Lubrication studies of smooth rubber contacts", The physics of tire traction - Theory and experiment 179 (1974)

[27] K.N.G. Fuller and D. Tabor: "The effect of surface roughness on the adhesion of elastic solids", Proc. Roy. Soc. Lond. A 345, 327 (1975)

[28] A. Schallamach, Proc. Roy. Soc. Lond. B 66, 386 (1953)

[29] A. Schallamach: "Friction and abrasion of rubber", Wear 1, 384 (1957)

[30] A. Schallamach, Wear 3, 1 (1960)

[31] A. Schallamach: "A theory of dynamic rubber friction", Wear 6, 375 (1963)

[32] A. Schallamach: "How does rubber slide", Wear 17, 31 (1971)

[33] A.I. Leonov, Wear 141, 137 (1990)

[34] Y.B. Chernyak, A.I. Leonov: "On the theory of the adhesive friction of elastomers", Wear 108, 105 (1986)

[35] K.L. Johnson, K. Kendall and A.D. Roberts: "Surface energy and the contact of elastic solids", Proc. Roy. Soc. Lond. A 324, 301 (1971)

[36] M. Kröger: "Adhesion of rubber on smooth and rough surfaces", Kautschuk Herbst Kolloquium Proceedings, Hannover, 8.-11. November (2004)

[37] P. Andersson: "Contact forces and noise generation during hastily separation of tyre tread blocks from road surfaces", In P.-E. Austrell \& L. Kari (Eds.), Constitutive Models for Rubber IV, Balkema (2005)

[38] H. Hertz, "Miscellaneous Papers", Macmillan, London, 146 (1896)

[39] G. Sperling, Eine Theorie der Haftung von von Festoffteilchen an festen Körpern, PhD Thesis, Technische Hochschule Karlsruhe, Germany (1964)

[40] R.C. Drutowski: "Hertzian contact and adhesion of elastomers", J. Lub. Tech., Trans. ASME Ser. F 91, 732 (1969)

[41] J. A. Greenwood, J. B. P. Williamson: "Contact of nominally flat surfaces", Proc. R. Soc. London A295, 300 (1966)

[42] B.B. Mandelbrot, The Fractal Geometry of Nature, W.H. Freeman, NY (1982) 
[43] J. Feder, Fractals, Plenum Press, N. Y., London (1988)

[44] R. S. Sayles, T. R. Thomas, Nature 271, 431 (1978)

[45] A. Majumdar, B. Bhushan, J. Tribology, 112, 205 (1990)

[46] Z. Radó, A Study of Road Texture and its Relationship to Friction, PhD Thesis, The Pennsylvania State University (1994)

[47] A. Schröder, Charakterisierung verschiedener Rußtypen durch systematische statische Gasadsorption, PhD Thesis, University of Hannover, Germany (2000)

[48] B.N.J. Persson, Sliding Friction: Physical Principles and Applications, Springer Verlag, Berlin, Heidelberg, N. Y. (1998)

[49] B.N.J. Persson: "Theory of rubber friction and contact mechanics", J. Chem. Phys 115 $\operatorname{Nr} 9(2001)$

[50] B.N.J. Persson and E.A. Brener, Phys. Rev. E 71, 036123 (2005)

[51] B. N. J. Persson, E. Tosatti, J. Chem. Phys. 112, 2021 (2000)

[52] B.N.J. Persson, U. Tartaglino, E. Tasatti and O. Albohr: "The sealing effect", Kautschuk Gummi Kunstst. 57, 532 (2004); ibid. Nature Mat. Lett. 1255 (2004)

[53] B.N.J. Persson: "Rubber friction: Role of the flash temperature", J. Phys. Condens. Matter 18, 7789 (2006)

[54] G. Heinrich, M. Klüppel: "The role of polymer-filler-interphase in reinforcement of elastomers", Kautschuk Gummi Kunststoffe 57, 452 (2005)

[55] G. Heinrich, M. Klüppel: "Recent advances in the theory of filler networking in elastomers", Adv. Polym. Sci. 160, 1-44 (2002)

[56] G. Heinrich: "Dynamics of carbon black filled networks, viscoelasticity, and wet skid behavior", Kautschuk Gummi Kunststoffe 45, 173 (1992)

[57] G. Heinrich, L. Grave and M. Stanzel; VDI-Berichte No. 1188, 49-67 (1995)]

[58] G. Heinrich and H.B. Dumler: "Wet skid properties of filled rubbers and the rubberglass transition", Rubber Chem. Technol. 71, 53 (1997)

[59] G. Heinrich: "Hysteresis friction of sliding rubbers on rough and fractal surfaces", Rubber Chem. Technol. 70, 1 (1997)

[60] G. Heinrich, M. Klüppel, und T. A. Vilgis: "Evaluation of Self-Affine Surfaces and their Implication to Frictional Dynamics as Illustrated with a Rouse Material", Comput. \& Theor. Polym. Sci. 10, 53 (2000)

[61] G. Heinrich and M. Klüppel, Kautschuk Gummi Kunstst. 57, 452 (2004)

[62] M. Klüppel, J. Meier and M. Dämgen: "Modeling of Stress Softening and Filler Induced Hysteresis of Elastomer Materials", p. 171-177 in P.-E. Austrell und L. Kari (Eds.), "Constitutive Models for Rubber IV", A. A. Balkema Publishers, Lisse, Abingdon, Exton, Tokyo (2005) 
[63] M. Klüppel, G. Heinrich: "Rubber friction on self-affine road tracks", Rubber Chem. Technol. 73, 578 (2000), and "Rubber Friction on Self-Affine Road Tracks", ACSMeeting, Rubber Division, Chicago, USA, 13.-16. April (1999)

[64] M. Klüppel, G. Heinrich: "Statistical Theory of Rubber Friction on Rough Surfaces", Proceedings: Deutsche Kautschuktagung, Nürnberg, 4.-7. Sept. (2000)

[65] M. Klüppel, A. Müller, A. Le Gal and G. Heinrich: "Dynamic contact of tires with road tracks", Paper No. 49, ACS Meeting, San Francisco, April 28-30 (2003)

[66] A. Le Gal, X. Yang and M. Klüppel: "Evaluation of sliding friction and contact mechanics of elastomers based on dynamic mechanical analysis", J. Chem. Phys. 123, 014704 (2005)

[67] A. Le Gal and M. Klüppel: "Modelling of rubber friction: A quantitative description of the hysteresis and adhesion friction", p. 509-514 in P.-E. Austrell und L. Kari (Eds.), "Constitutive Models for Rubber IV", A. A. Balkema Publishers, Lisse, Abingdon, Exton, Tokyo (2005)

[68] A. Le Gal and M. Klüppel: "Investigation and modelling of rubber adhesion friction on rough surfaces", Kautschuk Gummi Kunstst. 65, 308 (2006)

[69] A. Le Gal, L. Guy, G. Orange, Y. Bomal and M. Klüppel: "Modelling of Sliding Friction for Carbon Black and Silica filled Elastomers on Road Tracks", Wear, in press (2007)

[70] A. Müller, A. Le Gal and M. Klüppel, Kautschuk Herbst Kolloquium Proceedings, Hannover, 8.-11. November (2004)

[71] J. Schramm, Reibung von Elastomeren auf rauen Oberflächen und Beschreibung von Nassbremseigenschaften von Pkw-Reifen, PhD Thesis, University of Regensburg, Germany (2002)

[72] A. Müller, J. Schramm and M. Klüppel, Kautschuk Gummi Kunstst. 55, 432 (2002)

[73] S. Westermann, F. Petry, R. Boes and G. Thielen: "Experimental investigations into the predictive capabilities of current physical rubber friction theories", Kautschuk Gummi Kunststoffe 57, 645 (2004)

[74] S. Westermann, F. Petry, R. Boes and G. Thielen, Tire Technology International Annual Review, edited by UKIP Media \& Events, UK, 68 (2004)

[75] H.S. Carslaw, J.C. Jaeger, Conduction of heat in solids, $2^{\text {nd }}$ Edition, Oxford University Press (1980)

[76] P. Moldenhauer, M. Lindner, M. Kröger and K. Popp: "Modelling of hysteresis and adhesion friction of rubber in time domain", p. 515-520 in P.-E. Austrell und L. Kari (Eds.), "Constitutive Models for Rubber IV", A. A. Balkema Publishers, Lisse, Abingdon, Exton, Tokyo (2005)

[77] H. Montes, F. Lequeux and J. Berriot: "Influence of the glass transition temperature gradient on the nonlinear viscoelastic behaviour in reinforced elastomers", Macromolecules 36, 8107 (2003) 
[78] J. Berriot, H. Montes, F. Lequeux, D. Long, P. Sotta: "Gradient of glass transition temperature in filled elastomers", Europhys. Lett. 64, 50 (2003)

[79] L. Ladouce-Stelandre, Y. Bomal, D. Labarre, L. Flandin, Rubb. Chem. Technol. 76, 145 (2003)

[80] J. Foucard: "Rôle du pneumatique dans le phénomène d'adhérence", Bulletin de liaison des Laboratoires des Ponts et Chaussées 255, 11 (2005)

[81] J. Roth, PhD Thesis, University of Darmstadt, Germany (1993)

[82] H.H. Yang, M. Gespacher, L. Nikiel and C.P. O'Farell: "Traction predictive rubber compounds high-frequency viscoelasticity and tribology", Paper no.7 presented at the meeting of the Rubber Division, American Chemical Society, Dallas, April 4-6 (2000)

[83] J. Mc Namara, T. Alshuth and S. Jerrams, Kautschuk Herbst Kolloquium Proceedings, Hannover, 8.-11. November (2004)

[84] K.L. Jonhson, Contact Mechanics, Cambridge University Press (2001)

[85] J.G. Meier and M. Klüppel, Macromolecules, submitted (2007)

[86] M. Lindner, Experimentelle und theoretische Untersuchungen zur Gummireibung an Profilklötzen und Dichtungen, PhD Thesis, University of Hannover, Germany (2005) 


\section{Curriculum Vitae}

\section{Personal data}

Name

Date of birth

Place of birth

Nationality

Family status

Email

\section{Education}

1996

1996-1998

1998-2001
André Le Gal

09 October 1978

Ploemeur (F)

French

Married

andregilleslegal@yahoo.com

"Baccalauréat Scientifique" (Secondary School Diploma)

"Classe Préparatoire aux Grandes Ecoles", Lorient (F)

Physics and Engineering science

ISMANS, Ecole d'Ingénieurs, Le Mans (F)

Master Degree in Material Science and Polymer Physics

Professional experience

$2002-2006$

Research associate at the Deutsches Institut für Kautschuktechnologie (D.I.K.), Hannover (D) 